\title{
Retail Customer and Market Proclivity Assessment using Historical data and Social Media Analytics
}

\author{
by
}

Archika Sharma

A thesis submitted to the Faculty of Graduate and Postdoctoral Affairs in partial fulfillment of the requirements for the degree of

Master of Information Technology

in

Digital Media with Specialization in Data Science

Carleton University

Ottawa, Ontario

(C) 2020

Archika Sharma 


\section{Abstract}

Predictive analytics is a field which enables to predict the various aspects of a business. It offers vast prospects in today's business transformation by delivering an automated decision-making process. We focus our research on gaining insights into the retail market operations. Therefore, we introduce an integrated proclivity assessment model to examine the influence of consumer-market relationships. First, we perform a qualitative study to investigate the potential sales of the grocery market by utilizing the consumer's transactional history data released by Instacart. Our ensemble model for predicting purchase probabilities of the products performs better than the currently used baseline algorithms by achieving $88.84 \%$ accuracy. Based on the developed lexicon and rule-based sentiment analysis tool, our second proposed solution proficiently interpret the user propensity $(77.76 \%)$ towards grocery products by scrutinizing consumer tweets based on the user location. Finally, the third model which proposes an extended framework to detect the category of purchase intentions reflected by the consumers in their online reviews shows the highest $\mathrm{F} 1$ score of $94.17 \%$. If the information about the product experience is present explicitly in the review data, our customized technique can accurately segregate the different purchase intension labels (positive, negative, and unknown). Amalgamating the three distinct models, we present a web application as the use case that allows retailers to make improved market decisions. The integrated approach for mining consumer behavior and market tendencies can assist retailers to foresee the demand and adjust the supply chain of their business. Moreover, the practical use case of the framework can help in generating better schemes and effective promotional campaigns to maximize the revenues. 


\section{Acknowledgments}

It would not have been conceivable for me to compose this master's thesis without the assistance and encouragement of the generous people around me, to only some of whom it is feasible to give particular mention here.

Above all, I would like to express the deepest gratitude to my supervisor, Dr. Omair Shafiq, who introduced me to the field of data science. Without Dr. Shafiq, I would never have gotten the opportunity to study in such an esteemed institution. He continually and enthusiastically delivers his guidance and persistence which inspired me a lot during my research. His tremendous academic support and unique vision allowed me to launch the greater part of this thesis. His visionary brainstorming ideas and constructive feedback during the course term have been invaluable throughout all the stages of the research, which in turn helped me to improve my presentation, implementation, and writing skills. Thank you for believing in me and for giving me the necessary motivation when I started doubting myself. I could not have envisioned having a better advisor and mentor for my master's study.

I also appreciate other faculty members, academic staff of the School of Information Technology, for their support and diligence since the start of my degree. Thank you for always being available to provide directions and assistance.

Lastly and most of all, I am indebted to my family - my parents and my sisters, whose value to me only intensifies with age. Thanks for supporting me mentally and providing me a chance to explore myself professionally abroad. I am extremely grateful for their love, caring, and sacrifices to prepare me for my future. 


\section{Table of Contents}

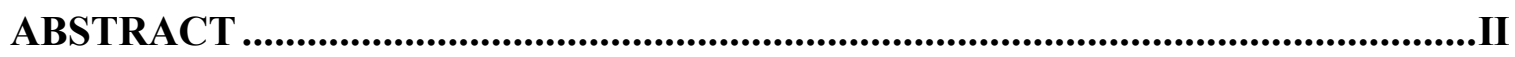

ACKNOWLEDGMENTS ........................................................................................ III

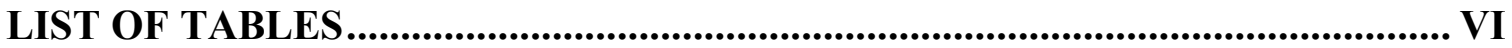

LIST OF ILLUSTRATIONS ............................................................................

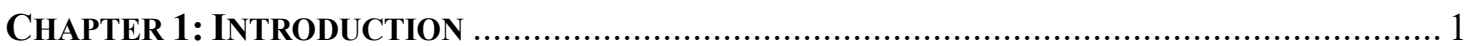

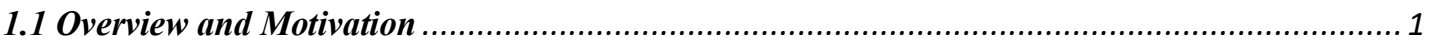

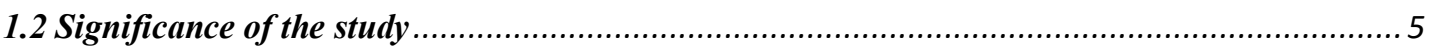

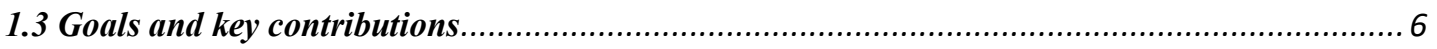

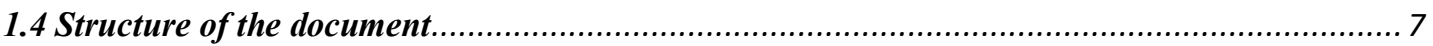

CHAPTER 2: DATA SET OF INSTACART FOR PREDICTION ANALYSIS .............................. 9

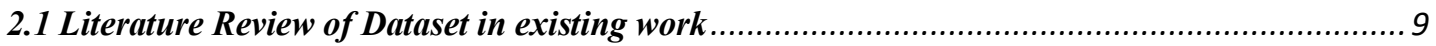

2.1.1 Datasets used in embedding-based recommender systems .................................................10

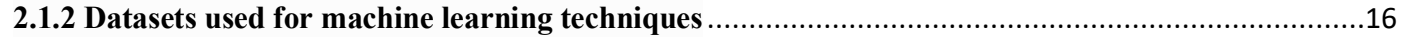

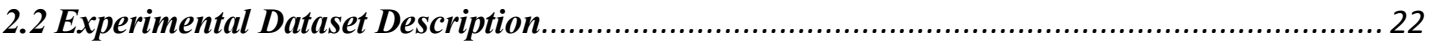

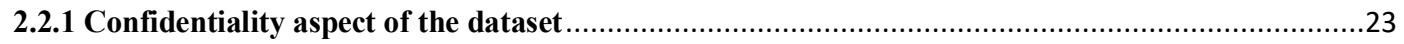

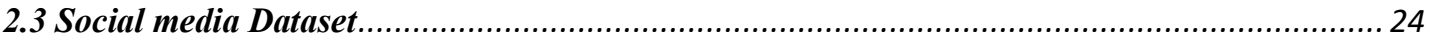

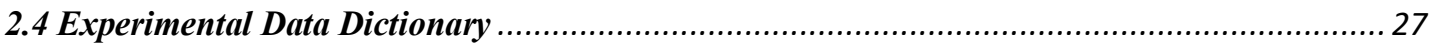

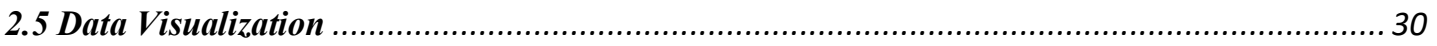

CHAPTER 3: REVIEW OF EXISTING WORKS..................................................... 40

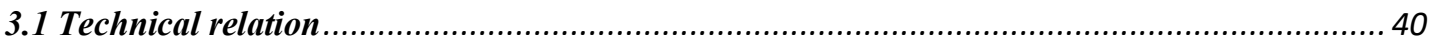

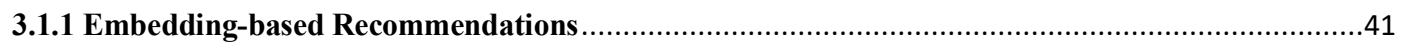

3.1.2 Recommender Systems based on transactional data .........................................................45

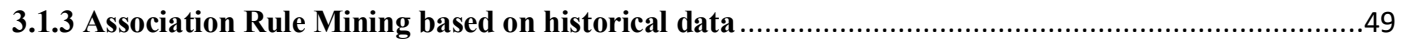

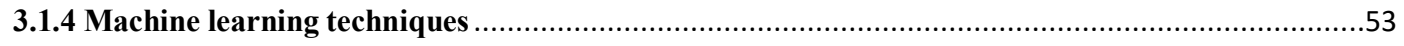

3.1.5 Other major analysis done on Grocery basket recommendation .........................................59

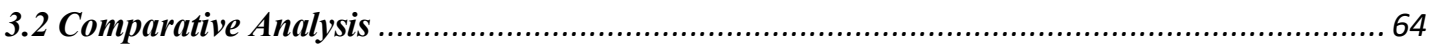

CHAPTER 4: EVALUATION OF RELATED WORK AND RESEARCH DESCRIPTION...............69

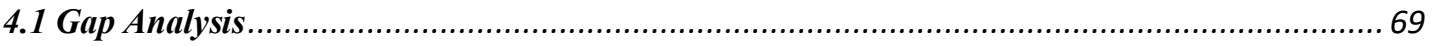

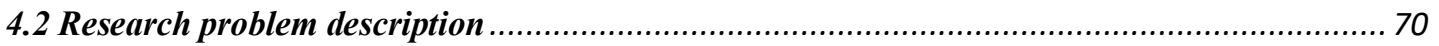

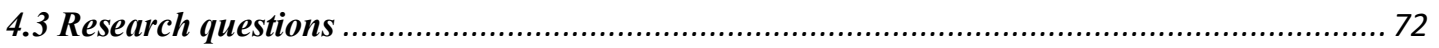

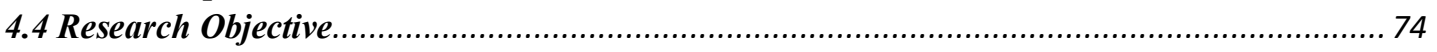

CHAPTER 5: EXPERIMENTS USING VARIOUS MACHINE LEARNING TECHNIQUES .............. 76

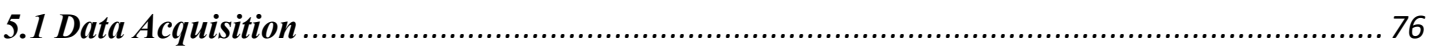

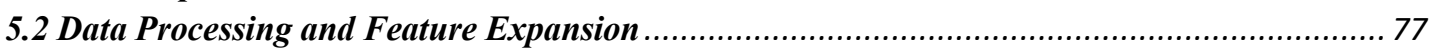

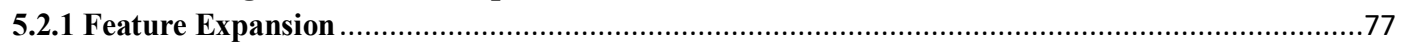

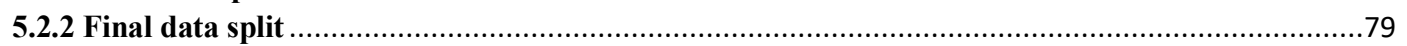

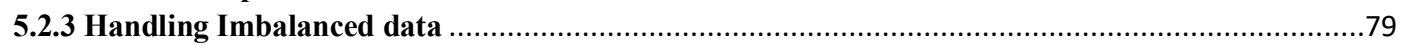

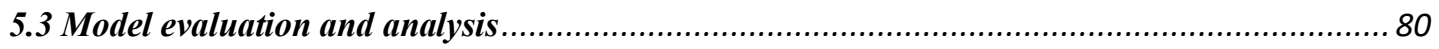

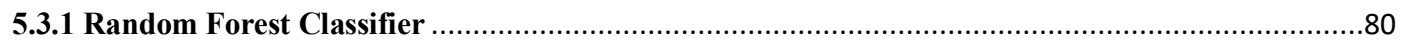

5.3.2 Extreme gradient Boosting (XGBoost) on Random forest classifier ........................................84

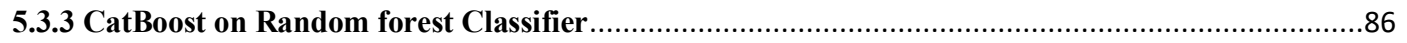

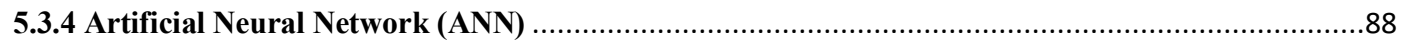

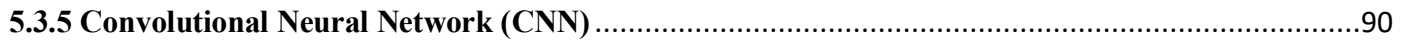

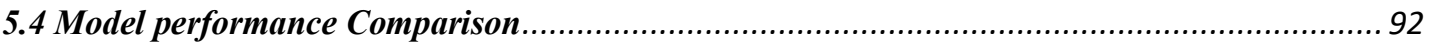

CHAPTER 6: DESIGN AND EXPERIMENTAL SETUP OF PROPOSED MODEL SOLUTIONS...... 101 
6.1 Model 1: Purchase probability prediction using historical data ......................................... 101

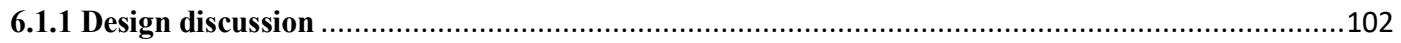

6.1.2 Technical design of Segment 1

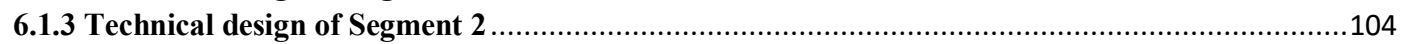

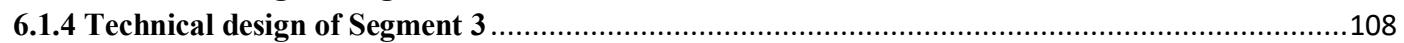

6.2 Model 2: Consumer propensity assessment using Twitter data.............................................. 109

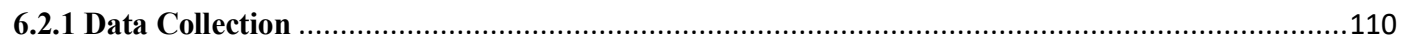

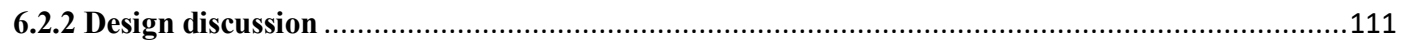

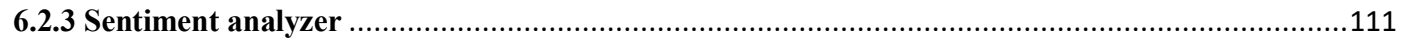

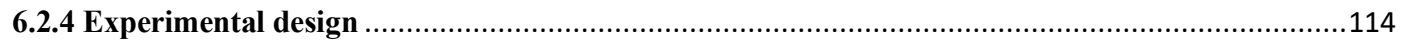

6.3 Model 3: Purchase intention detection based on online reviews.......................................... 115

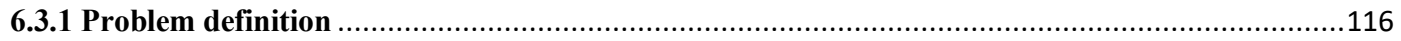

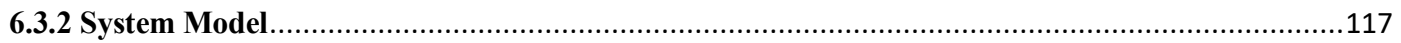

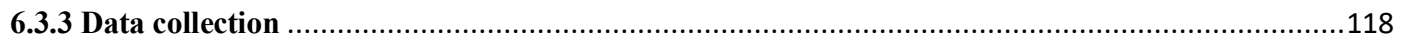

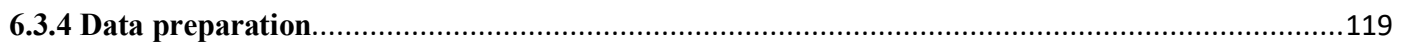

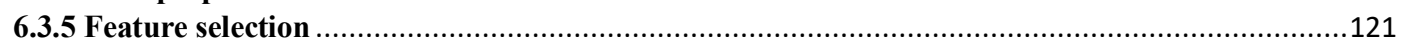

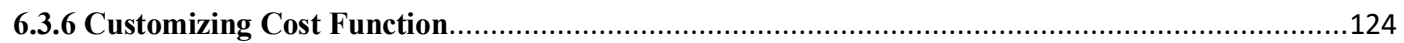

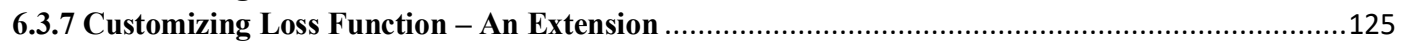

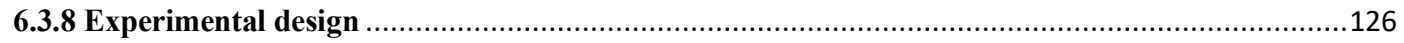

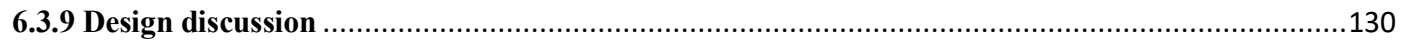

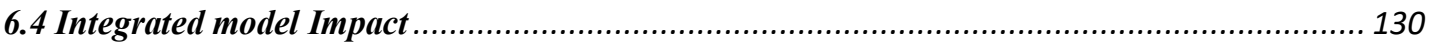

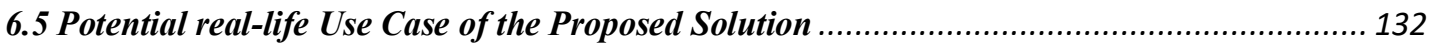

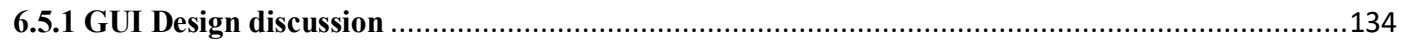

6.5.2 Potential of the Use case

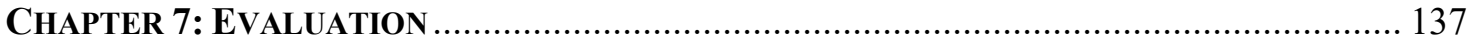

7.1. Model 1: Purchase probability prediction using historical data........................................ 137

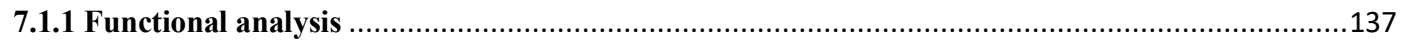

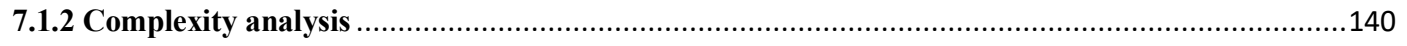

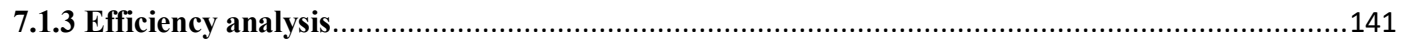

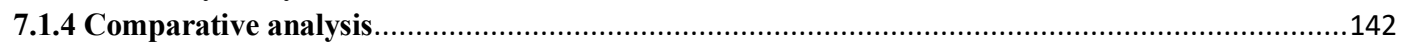

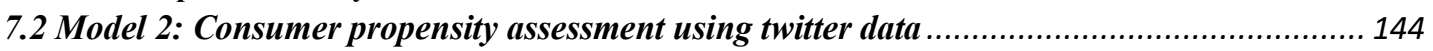

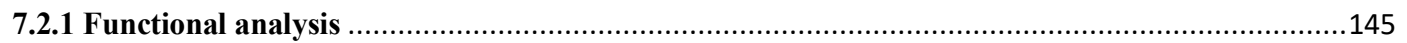

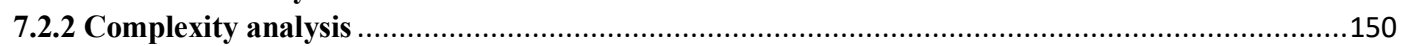

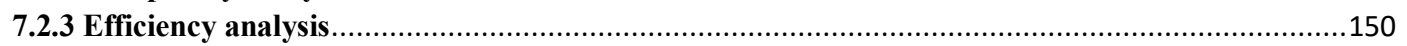

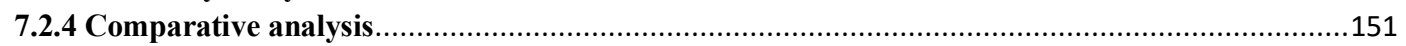

7.3 Model 3: Purchase intention detection based on online reviews........................................... 153

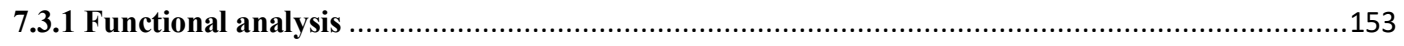

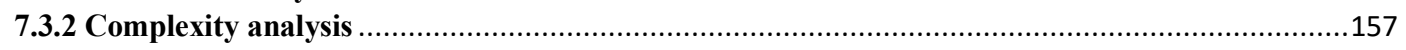

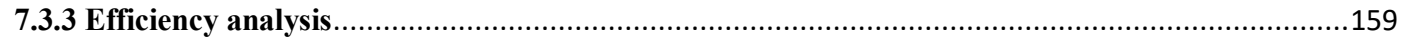

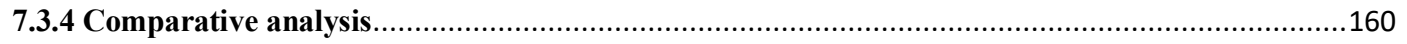

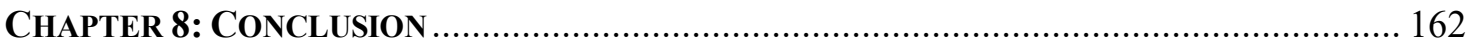

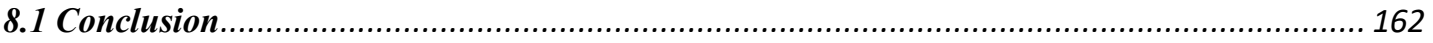

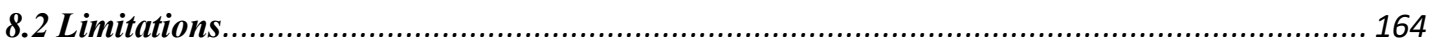

8.3 Future Work

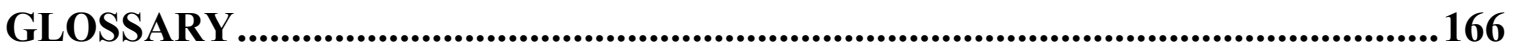

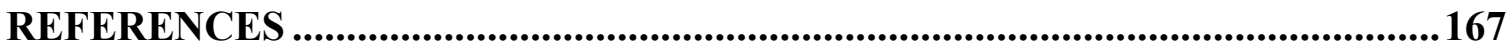




\section{List of Tables}

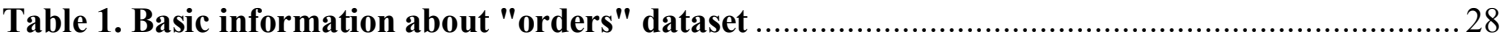

Table 2. Basic information about "products" dataset ..............................................................29

Table 3. Basic information about "order_product" dataset ......................................................29

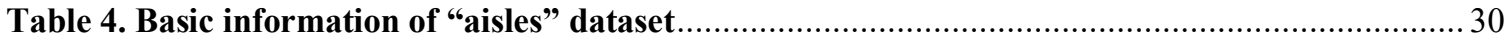

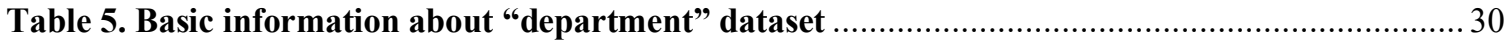

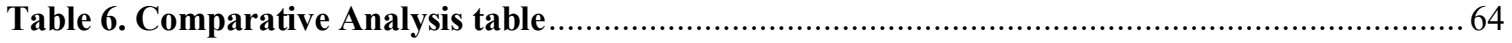

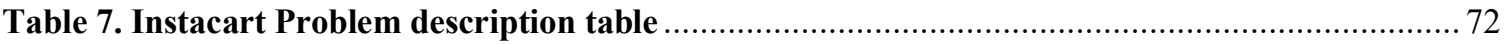

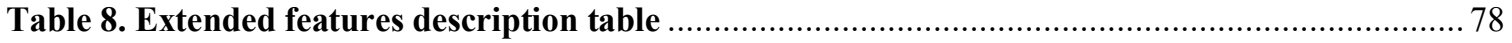

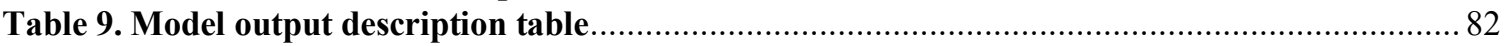

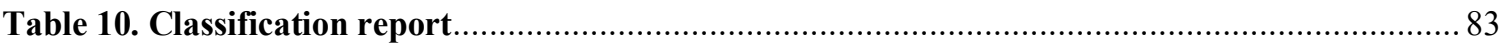

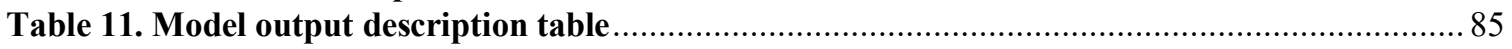

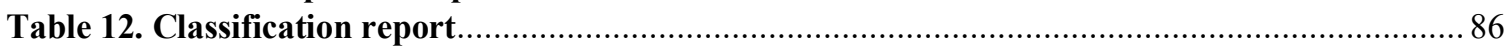

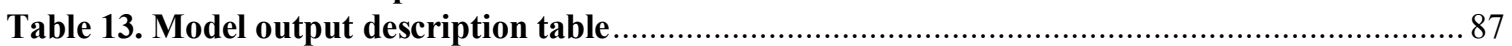

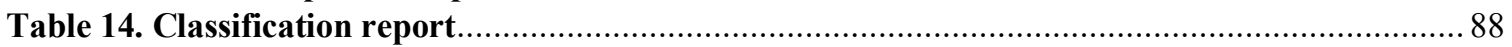

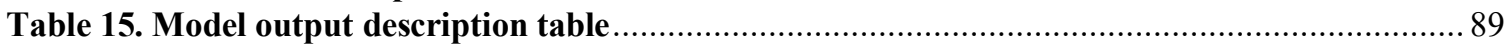

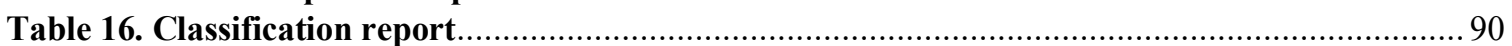

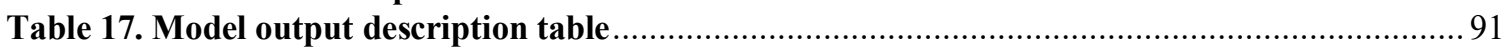

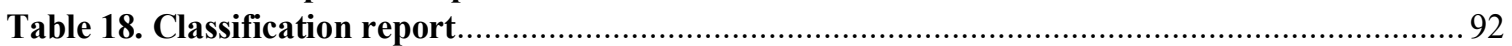

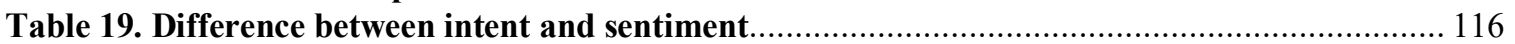

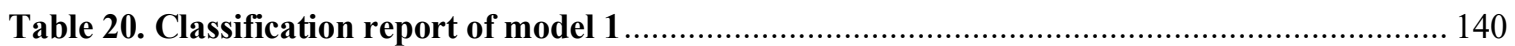

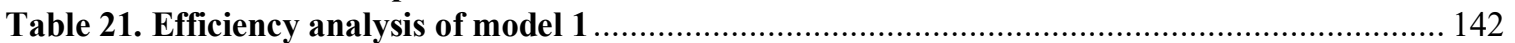

Table 22. Comparison of the proposed solution with related work ......................................... 143

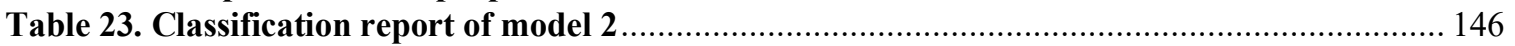

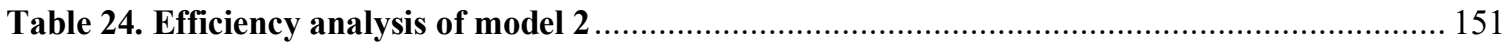

Table 25. Comparison of the proposed solution with related work ...................................... 151

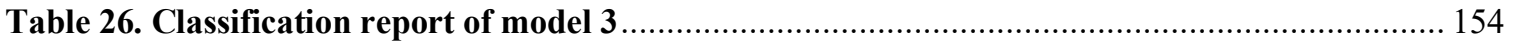

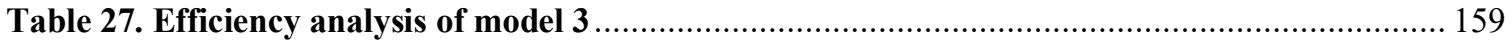

Table 28. Comparison of the proposed solution with related work ..................................... 160 


\section{List of Illustrations}

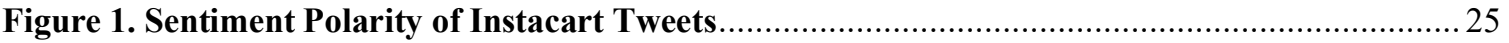

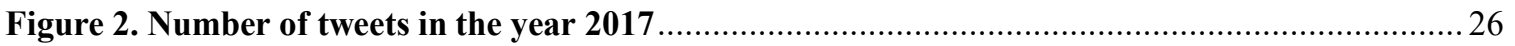

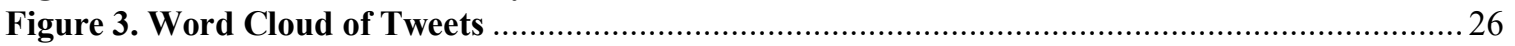

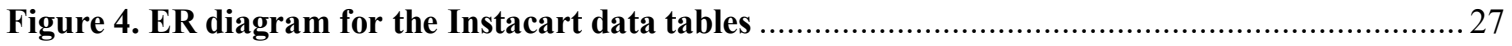

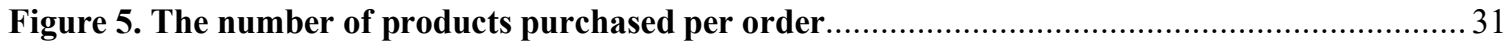

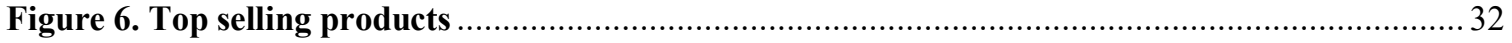

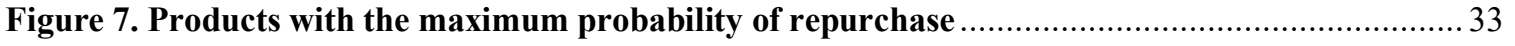

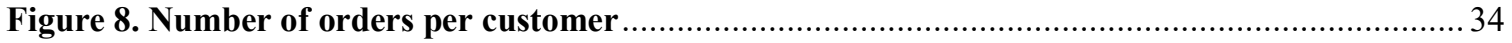

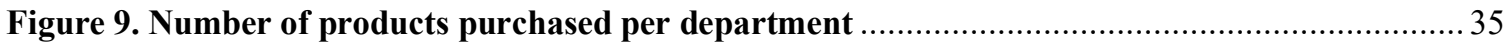

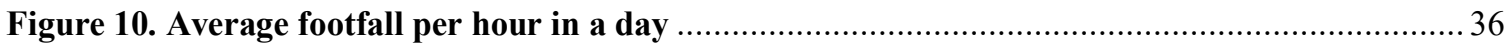

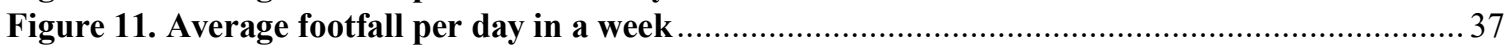

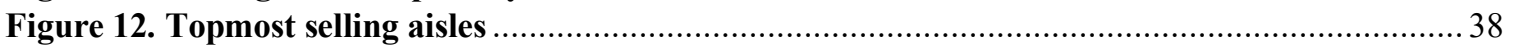

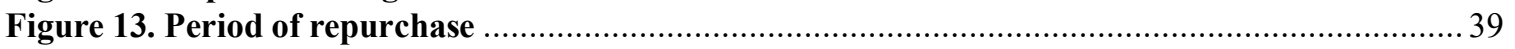

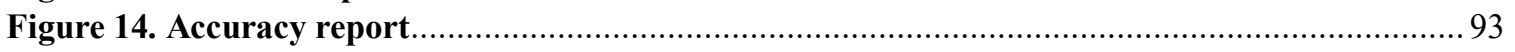

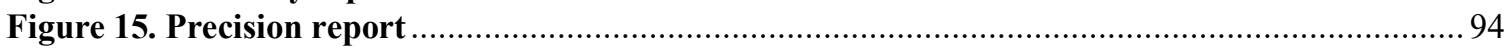

Figure 16. Area Under the curve report .............................................................................. 95

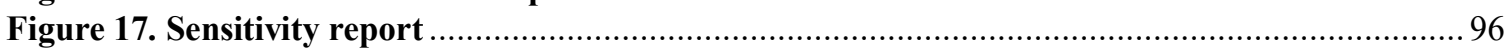

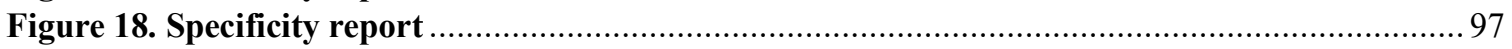

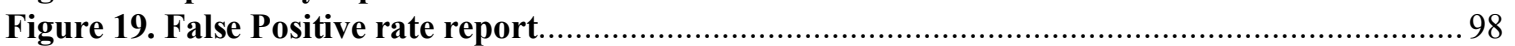

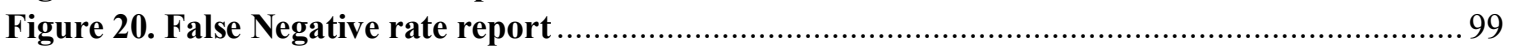

Figure 21. Detailed technical design diagram (Model 1) ........................................................... 104

Figure 22. Detailed Technical Design of Procedural Block 1 .................................................. 105

Figure 23. Technical Design of Procedural Block 2 ......................................................... 106

Figure 24. Technical Design of Procedural Block 3 .......................................................... 107

Figure 25. Detailed Technical Design diagram (Model 2) ................................................ 110

Figure 26. Detailed Technical Design diagram (Model 3) ..................................................... 118

Figure 27. Integrated proposed solution..................................................................... 131

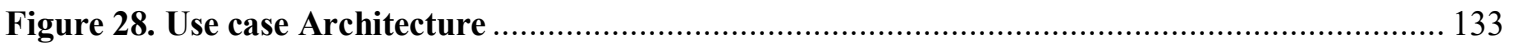

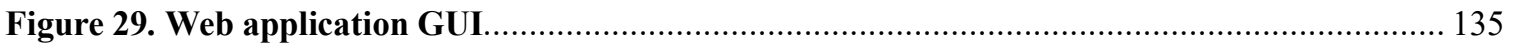

Figure 30. How does ensemble models affect Confusion matrix results ............................... 138

Figure 31. How does ensemble models affect the ROC curve ............................................. 139

Figure 32. Distribution of sentiments across all Tweets ................................................. 145

Figure 33. How do text classification models affect Evaluation results ............................... 146

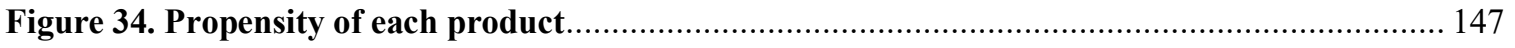

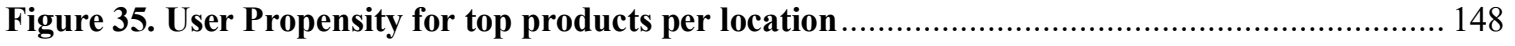

Figure 36. User Propensity for "Zucchini”" per location ............................................... 149

Figure 37. User Propensity for top products at "Toronto" location ...................................... 149

Figure 38. Negative Purchase intent assessment ........................................................... 155

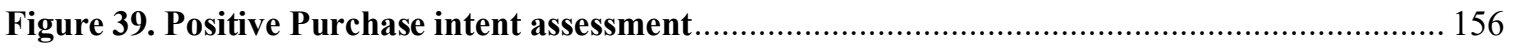

Figure 40. Unknown Purchase intent assessment........................................................... 156 


\section{Chapter 1: Introduction}

The purpose of this chapter is to establish the ground which in turn leads to understanding the qualitative theory and practical impact of this thesis. In the first section, we present an overview of the area under consideration along with research motivation. Then, we discuss the significance of the study along with the brief introduction to the key contributions, while the structure of the document is present in the last section of this chapter.

\subsection{Overview and Motivation}

Retail business is growing at a high pace and so is the demand for products in the market (Deloitte Touche Tohmatsu Limited, 2003). A huge number of transactions are occurring daily in this century (Rigby, D.K, 2019). Every purchase history varies in terms of culture, geographical locations, and in terms of consumer etiquette too. Besides, social networking sites provide a platform for users to express their responses towards any consumed goods or services provided by the company (Atouati, S. et al., 2020). In the past, to analyze the behavior and understand the need of customers, researchers used statistical surveys and manual data collection (Chrysochou, P., 2017). Modern firms endeavor to comprehend their database using mining techniques to extract knowledge which will help them capitalize on profitability and optimize maneuver for both their internal and external environment. To provide large profits from future sales and generate the maximum number of customers is one the major strategy in focus nowadays. To achieve the same and expand the current market, Information technology provides various problem solving and decisionmaking activities. 
Most studies have focused on recommending items to the consumer based on previous purchase behavior, where each product is suggested distinctly (Giering, M., 2008; Mohan, A. et al., 2019; Schafer, J. B. et al., 2001). In most situations, users often purchase multiple products at a visit to an online store (Bai, T. et al., 2018). The most crucial task of business analysis is the next product recommendation (Gatzioura, A., \& Sànchez-Marrè, M., 2014; Wang, P. et al., 2014), which is defined as to predict the next few products that the user would likely buy.

Basket analysis (Efrat, A. R., \& Gernowo, R., 2020; Kabasakal, I., 2020) is a longstanding task in the area of machine learning. Association rule mining (Agrawal, R. et al., 1993; Videla Cavieres, I. F., 2014; Suharjo, R. A., \& Wibowo, A., 2020) has been the dominating state-of-the-art in terms of retail analysis for a couple of years. The principle of this algorithm involves computing the possibility of buying additional products inferring from frequently occurring patterns in past observations of purchase history. The robustness and computational cost of this algorithm became the main reason to invent modern approaches to tackle the above problem.

Many research papers have significantly proven that transactional data of purchase in the form of relational databases could precisely envisage the patterns of dynamic customer behavior and the other substantial aspects of the retail business (Mohan, A. et al., 2019; Kouki, P. et al., 2019; Chrysochou, P., 2017; Lu, H. et al., 1998). Plenty of academics provided substantial results while evaluating online user-generated data (Kumari, P., \& Haider, M. T. U., 2020; Da Silva, N. F. et al., 2014). Many online purchasers share their personal experiences related to the commodities provided by retailers. While assessing the field of computational social sciences (Joshi, R., \& 
Tekchandani, R. 2016; Liang, P. W., \& Dai, B. R. 2013), researchers have shown that data resulting from user-generated tweets can be used to predict the movie revenues and sales of digital devices. Centered on the idea of Vatrapu et al., 2015, we aim to build a propensity model by assuming that digital interactions associated with a product influence the attention of the consumers and hence boost the market value of that product.

In parallel, there have been many efforts made to understand the patterns of the customer's visits in a store (Hanke, J. et al., 2018; Walter, F. E. et al., 2012; Keller, T., \& Raffelsieper, M., 2014), others aim to predict customer intentions in real-time (Hamroun, M. et al., 2015; Fernandes, R. F., \& Teixeira, C. M., 2015). As a result, many researchers exploited different machine learning techniques (Gupta, V. et al., 2014; Ding, X. et al., 2015) for recognizing when a customer is ready to buy or expresses interest to buy a product in their reviews or comments. Quite often the focus of the research is single variate (if purchase intent is present or not). However, we aim to identify the specific label of the purchase intent (Positive, negative, or unknown) conveyed by the user on an online platform.

Our research could be an exemplar to depict how machine learning can be applied to retail business where no user interaction detail is available explicitly. The task is to predict the selling probability of each product along with the analyzing customer's purchase intentions towards the product by utilizing online user-generated data. Concisely, we aim to understand the proclivity of the customers relating to the retail market and the other way around. So, in other words, proclivity refers to the inclination of the subject towards a particular object, wherein our case subject and object refers to consumers and retailers. 
The data used and the algorithms used on it would allow grocery suppliers to revolutionize the process as to how customers discover and purchase groceries. Based on abundant previous research discussed in chapter 3, we come up with three major research questions and propose a comprehensive solution followed by a thorough assessment that aims to resolve the research questions which are discussed in chapter 4 .

Our research work concentrates on investigating and defining a novel predictive structure to generate the probabilities associated with the purchase of each item present in the online-stores and to comprehend the dynamically changing customer's intentions. The CRISP-DM (Cross Industry Standard Process for Data Mining) methodology (Wirth, R., \& Hipp, J., 2000) will be used as the data mining process to organize and conduct the research of this area efficiently.

The framework demonstrates the structured procedure and techniques for analysis, exploration, modeling, mining, implementation, and delivery (Shearer, C., 2000). The stage of delivery is not covered under this study, which could be planned later.

To implement the research idea as explained above, we commenced the work plan by obtain the visual findings from the raw data set of Instacart which will be later contributed to extract the new features for our data modeling practice. We also investigated the social media data associated with Instacart to comprehend human behavior correlated with the most re-purchased products.

Following that, we processed the data to fit it into the various machine learning models. The data processing and feature expansion in chapter 5 configure the data design for the proposed solution by assessing the results processed by five distinct machine learning experiments. All the models used a CPU-based training procedure. 
Next, by performing a comprehensive evaluation of models such as functional analysis and classification report comparison, we conclude various findings that are worth leveraging more in-depth research. Hence, we introduce the ensemble learning-based model to influence the final probability results. After exploring the historical data, we establish an effective framework that analyzes user propensity and comprehends their purchase intention labels by utilizing online generated data. The evaluation results have proved that our proposed frameworks are significantly helpful to interrogate the dynamic structure of the retail market and its consumers. Our model designs are then integrated into a user-friendly interface which could then be used to elevate market strategies.

\subsection{Significance of the study}

This research work will provide an understanding of the consumer's behavior and intentions to buy a product within the grocery shopping context. The preliminary study investigated the impact of feature expansion on the model performance that interrogates the re-purchase probability of the product. Next, our research piece identifies the impact of ensemble learning which boosts the overall prediction results.

The important practical contribution of the study lies in determining the user propensity level and detecting the purchase intension manifested through online posts and reviews. Another significant aspect lies in identifying how to project these influential model prognostications to the market retailers to make effective decisions concerning promotions and business marketing.

Consumers may also obtain benefits from expanded product choices at feasible costs to meet their needs. Factors in elements such as high demand location can provide 
product variety and can help in targeting the cost-effective market (Peter, J. P. et al., 1999). Offering promotional discounts and optimizing manufacturing efficiency will affect the consumer's mind, resulting in loyalty towards the brand. (Chaudhuri, A., \& Holbrook, M. B., 2001).

\subsection{Goals and key contributions}

This section incorporates the specific goals and incentives to achieve them. There are four primary goals of this study:

\section{a) Multiple experiments using machine learning and deep learning techniques}

To pragmatically test the eminence of features extracted from the raw data labels to predict the re-purchase probabilities of the products by examining the Instacart's transactional data. We aim to use the ensemble method to compare the edging results of five different machine learning algorithms.

\section{b) The historical data evaluation model}

To develop a conceptual framework based on ensemble learning that integrates three machine learning algorithms which empirically orients model standards to yield superior results. We exploit neural networks along with gradient boosting decision trees in order to accomplish this purpose.

\section{c) Propensity assessment model}

To understand how social media plays a vital role in comprehending the inclination of the user's interest in grocery products by studying social media posts. 
Specifically, we target twitter data for this task to collect the data for model evaluation. Moreover, our solution set an example that social media not only just convey sentiments but can also be used to assess consumer's expectations and needs.

\section{d) Purchase intention detection model}

To distinguish the user intentions concerning a particular product in the market. The primary data is collected through a grocery selling website utilizing web scraping. We develop an extension model of Atouati, S. et al., 2020 to examine the customer relationship with products in terms of their buying intension. The model segregates the purchase intent directed by the customer in three labels: Positive, Negative, or Unknown.

\subsection{Structure of the document}

This thesis consists of eight main chapters. First, we introduce the research motive, significance, and summary of key contributions in the first chapter, we review the data chosen for this study within six sections of chapter 2 . We illustrate the literature foundation of the chosen dataset in comparison to the work that has already been done in this field. Building on that, we extensively describe how our dataset was collected illustrating the confidentiality aspect of the transactional data. We also visualize the various labels and features present in different tables of the Instacart dataset.

The next chapter 3 reviews the literature regarding the product-customer relationship concerning different research methodologies applied so far. We also provide a thorough comparative description of the related work concepts and processes. In chapter 
4 , we present the gap evaluation analyzed in chapter 2 along with a comprehensive problem description of the research work. We also introduce the research questions as well as conceptual framework objectives.

In chapter 5, we present an in-depth analysis of data pre-processing which leads to feature expansion and data balancing. We also examine the five distinct ensembled machine learning algorithms for re-purchase probability prediction (Random forest, XGBoost, CatBoost, Artificial neural network, and Convolutional neural network) and their evaluation statistics in this chapter.

In chapter 6, we first analyze the influence of the ensemble technique to enhance re-purchase probability predictions. Next, we assess the predisposition of twitter users based on the most re-purchased grocery products of Instacart. In the third part of chapter 6 , we identify user intentions based on online reviews data which basically provides an extension to the approach in Atouati, S. et al., 2020. We integrate three distinct solutions in the next fragment of the chapter. In the end, after model compilation, we present a practical use case of our solution which provides an interactive structure to explore the relationship between products and promotional offers. The seventh chapter displays the metric scores acquired by the three model solutions and examines the cost of each algorithm along with brief result comparison analysis with previous work. Concluding remarks of our findings, and future directions, are enlisted in the 8th and the last chapter. 


\section{Chapter 2: Data Set of Instacart for prediction analysis}

In this chapter, we review the dataset selected for this study and how it correlates to the literature foundations within five sections.

The first section explains the survey of existing work done in the field of retail business data mining to expand and strengthen their relationships with customers as well as the main strategies of cross-selling techniques.

Next, we introduce the reader to the high-level description of the dataset provided by Instacart along with measures taken to maintain the confidentiality of released data as an open-source.

In section 3, we conduct a social media data evaluation to understand the requirement of the study through the consumer's standpoint.

In the next section, we will define different features and variables available in various tables of the dataset along with its datatype descriptions. Lastly, we present the data structure and in-depth visualization of the data to infer new knowledge about the transactions done.

\subsection{Literature Review of Dataset in existing work}

Enormous amounts of data get generated daily from the past two decades, out of which almost $90 \%$ of the data that existed in 2018 was created within the prior two years (MediaPost, 2018). According to Manyika, J., 2011, the term "Big Data" refers to the set of data whose size surpasses the capabilities of typical software in terms of managing and analyzing. Former Google CEO, Eric Schmidt commented that "There were 5 exabytes of 
information created between the dawn of civilization through 2003, but that much information is now created every two days, and the pace is increasing".

In this section, we list the literature evaluation of data collection. Before researching the previous works in the product personalization domain for technical instructions, we first go through the related work of datasets that have previously been used for comparable tasks which will provide a direction to collect data, and how it will benefit the retail market analysis.

\subsubsection{Datasets used in embedding-based recommender systems}

Data is generated based on various factors, such as the user's location, cultural change, interest, daily routine. In this section, we highlight some works done to mine the large dataset of users based on daily transactions to extract the facts about the needs of the consumer. Some researchers collaborate with various organizations in order to be able to work on their dynamic set of data and provide back analysis results.

Overview: Kouki, P. et al., 2019 built a recommender system and tested it on online transactional data of Home Depot. They picked two datasets, one from the Bath root category (487 products) and the other from the Patio root category (369 products). The authors processed 129,060 records of the relationship for Bath and 23,433 for Patio from domain experts.

Strength: The authors targeted the collection problem in three separate segments. They used the combination of transactional data, content information for products, and domain knowledge. Products in the dataset were integrated in order to share a similar level of functionality according to their hierarchies. They randomized the order of presentation of products to eliminate biased results. 
Weakness: To evaluate the quality of their proposed solution, they manually collected 5 product recommendations from each validator as a relevant suggestion or not. Collecting data manually is time-consuming and requires intense efforts. Both datasets contained information on products and their domain knowledge such as hierarchical identification details of a product. While they did propose a novel technique to generate the set of products with a similar theme, instead of using historical data, we prefer to use recent data with many large recordsets so that we keep the evaluation of results up to date.

Overview: While processing bundle information of products to develop a recommender system, Wölbitsch, M. et al., 2019 worked on the dataset containing half a million records from 20 stores of 4 different cities. The data is collected for approximately a time frame of 2 years i.e. November 2016 to December 2018. As their main aim was to analyze the products that are often bought together, they rejected the records which contain single product purchase. This reduced their dataset to 146,720 records out of half a million.

Weakness: The main issue in their dataset was occurred due to the limited data availability and data sparsity while generating bundle purchase information as they discarded the basket information of single item purchases. Furthermore, duplicate shopping baskets were removed to process unique data records. The dataset of shopping baskets was collected from four different cities which incorporates the issue of data sparsity. According to the Jaccard similarity coefficient, the item similarity between different cities was calculated 0.51 and could differ more due to change in local conditions and demographics.

Strength: The unique feature of their research was data augmentation. To eliminate the dilemma of limited data, authors expanded the available data of shopping baskets which contained multiple products. The new extracted baskets contained a pair of products or 
triple products and hence were added to the training set of the data. Their experimental measurements and conclusions confirmed that the approach of augmentation showed a consistent improvement of recommendation performance.

Overview: Mohan, A. et al., 2019 worked on creating a recommender system integrated with business intelligence application SmartEmma. They exploited various ETL tools to extract data from the source in the structure of databases. Key tables of their database included Customer/Users table, which contained basic information about the users, such as birthdate, gender, transaction date.

Strength: The store table consisted of details about the retailers, location of the store, scheduled business hours. Next, the product table contained information like prices, and a lot of details were recorded to be missing for this database table. The final table of Orders/Transactions contained transaction details associated with the user. A solitary transaction of a certain order was then further divided into multiple minor transactions associated with different retailers.

If we plan to deploy our study in a real-life business platform and how it will enhance the current scenario of Instacart, then this research procedure is worth referring to. Such type of detailed dataset with detailed statistics could lead to better knowledge extraction and sophisticated accuracy.

Weakness: The key data tables for this research were generated based on the information provided by the retailers without much alteration. As mentioned by the author, the transactional data used for the study is significantly smaller which is prone to produce skewed results in terms of calculating similarity function. 
Overview: Barkan, $O$., \& Koenigstein, $N$., 2016 evaluated the word2vec approach in order to introduce a method that inferred item to item relationship (item2vec method). To exploit their novel technique, they evaluated the method on two private datasets from Microsoft stores. The first dataset contains 9 million records of user's interaction in terms of songs played by them. This dataset captured the user-artist relationship which means the song of a specific artist played by the user. Mathematically, the dataset of Microsoft Xbox Music services contained $732 \mathrm{~K}$ users and $49 \mathrm{~K}$ different artists.

Strength: The strength of their work was that they applied both qualitative and quantitative results. The neural embedding approach followed by the authors was capable of correlating the items without the user information. Their application tends to recommend the target item by exploiting the contextual information of the item.

Weakness: The second dataset that researchers exploited was of purchases done at Microsoft stores. This dataset included 379K orders of 1706 diverse items. However, the buying information on the items did not contain the specifications of the user. Hence, the dataset was weaker in providing local conditions of the user which could have been useful to deeply mine customer conduct.

Overview: A novel neural language-based algorithm was proposed by Grbovic, M. et al., 2015 that leverage user's purchase history. They targeted user's email addresses to focus on buying behavior and to provide more personalized recommendations. To develop the prediction model, they collected training data of 280.7 million purchase records in terms of email receipts which included 29 million distinct users. The purchase history dataset was from 172 different commercial websites. 
Strength: To provide a deeper insight into the purchased data, Grbovic, M. et al., 2015 segregated user information in terms of demographic backgrounds, age, gender, location by keeping the user IDs anonymous. Such kind of detailed dataset is helpful if we aim to explore the buying behavior of the customer based on age ranges, gender specifics, the difference in online or in-store buying patterns.

Weakness: The authors presented a novel technique to target product personalization on large-scale data by studying Yahoo emails. However, their framework focused on userproduct interaction which tends to change dynamically. Hence, it required frequent data updates to be injected for better model performance.

Overview: Grbovic, M., \& Cheng, H., 2018 proposed a novel method that consists of the low-dimensional representation of users and house listing to provide real-time personalized recommendations for the Airbnb marketplace. For the collection of training listing embeddings, 800 million click sessions of anonymous logged-in users were evaluated.

Strength: Click sessions that contain 2 or more clicks were considered based on 30 minutes of inactivity rule. Evaluation results of click sessions that ended with booking yielded 4.5 million Airbnb listing. Moreover, before the online deployment of the framework, they performed rigorous offline testing of the embedding system to eliminate the worst-case scenario.

Weakness: While training the home listing dataset, the authors initialized each listing ID with a vector to provide dimensionality. Although, in order to collect live user preferences, training data set is required to be updated daily in the form of windows while discarding the oldest session records from the dataset. This procedure of re-training the embedding list daily compromises the performance rate of the model. 
Overview: Vasile, F. et al., 2016 focused on 30 Music open datasets to leverage their research of the Meta-Prod2vec approach with an aim to synthesis better recommendations using metadata information of the items. Authors evaluated publicly available dataset that contains the collection of playlists from Internet radio stations through their API last.fm.

Strength: The dataset of their experiment consisted of playlist sessions of 100K users which included $433 \mathrm{k}$ songs associated with $67 \mathrm{k}$ distinct artists. Their research work aimed to recommend the next song to the user by evaluating previously played songs which brought in the concept of user personalization. The metadata of the song contained information such as artist information, album name.

Weakness: In Vasile, F. et al., 2016, the item metadata were integrated with the items to normalize the embedding in the model. There were some minor limitations located in their data source and analysis method. In their approach, each attribute group did not have independent learning embedding vectors. Moreover, their experiment method was concentrated only over a single feature i.e. Singer ID.

Overview: While working on the unsupervised embedding of users and items, Wan, M. et al., 2018 proposed a method triple2vec to learn both complementary and compatibility relations. To ensure the producibility of their proposed approach, the authors considered two proprietary datasets and two public datasets.

Strength: Proprietary datasets used in the experiment consisted of transactional data named as MSR-Grocery (WA) and MSR-Grocery (UT). The dataset included 12 months of transactional data of 360k users. Latter proprietary dataset i.e. MSR-Grocery (UT) included 8 months transactional data and was collected from two middle-sized grocery stores of Salt Lake City area that included more diverse consumers than the former one. 
Weakness: Transactional data of Dunnhumby collected over two years was exploited as public data in their experiment. Their research used Instacart transactional data as a second public dataset, the same data source as of our research, thus their conclusions are significant for our research. The baseline model that they represented to serve personalized item recommendations at large-scale demands increase in time required for training deep embedding due to outpouring data size cumulated by increasing online grocery shopping demand.

\subsubsection{Datasets used for machine learning techniques}

In the field of data science, one of the most essential marketing research is based on customer behavior predictions. The uncertainty in the objective is very high and the demands of the consumers deviate frequently. Overview: Despite all these qualms, Kumar, A. et al., 2019 investigated the features associated with the customer's product repurchase practice. With the use of ensemble machine learning techniques, the authors concluded that the AdaBoost classification model yielded $97.58 \%$ accuracy in predicting the intentions of repurchase.

Strength: To experiment, authors collected their data by executing the online structured questionnaires in metro cities of India due to advanced internet users and higher online purchasers. These surveys accepted the answers on a scale of 1-5. Data collection was also used offline utilizing judgment sampling. In total, they collected 430 questionnaires, out of which 310 questionnaires were collected online and 120 from the survey done offline.

Weakness: The main set back of their dataset was that they considered consumer personal attributes following their online shopping attributes, ignoring the factors that could 
generate maximum revenues to the company such as interactive website design, the ranking of the service provider, feedbacks of the product provided by consumers.

Overview: To comprehend the user's purchasing patterns, XingFen, W. et al., 2018 proposed a solution that meaningfully provided personalized suggestions and instructions to skim consumption behavior.

Intending to predict user's purchase of a specific product in the future, authors analyzed the original dataset of an e-commerce website collected in the year of 2016 for three months. They processed the raw data with $51,289,796$ records that depict different activities of $1,05,321$ users.

Strength: Authors exploited logistic regression with the XGboost algorithm processed on more than 50 million data records, out of which $60 \%$ was randomly considered as the training set, $20 \%$ as verification set, and rest as the testing set. According to the experimental results of their study, logistic regression on XGBoost yielded more feasible results than considering them individually.

Weakness: In their study, the authors used the XGBoost model for the transformation of features and generated new feature vectors. But parameter searching using XGBoost is considered time-consuming practice (Jiang, Y. et al., 2019). Moreover, user behavior prediction demands a dynamic framework that molds the outcomes depending on the contemporary situation of the market.

Overview: Sakar, C. O. et al., 2019 projected a system that analyses the online shopper's behavior dynamically. Their study aimed to generate browsing users that intend to purchase an item but expected to leave the web page in the prediction horizon. Such users were provided with offer content to increase conversion rates. 
Strength: Authors used a specific dataset of 185,000 Web pages visited in 9800 sessions of 3500 visitors for recurrent neural network-based analysis. The field of URL information in the dataset was used to extract features such as product view, administrative operation, and information acquisition operation. These feature vectors were then fed to a Long shortterm memory-based recurrent neural network (LSTM-RNN) model to estimate the probability of the users that will abandon the webpages without placing their orders.

Weakness: However, before the final purchase, online users tend to match the prices of the product on various channels. The user's intention of purchase based on leaving the website without placing their order could lead to many other factors (Padmanabhan, B. et al., 2001), and hence, providing personalization from incomplete or vague data could lead to weak outcomes.

Overview: Tahiri, N. et al., 2019 worked on a novel machine learning model compiled in python 3. Their proposed solution aimed to predict the purchase-repurchase of the products by the consumers. They also introduced a personalized recommendation product list based on consumer's purchase history.

Strength: The datasets used to carry out their anticipated machine learning technique were obtained from the MyGroceryTour.ca website from the year 2017. The real dataset contained 374 users that were assigned random user Ids. The actual dataset contained 49,684 distinct products but only 1000 of them were considered in their model. The unique feature of their dataset was the "Reorder" value ( 0 or 1$)$. Reorder value was assigned 1 if the product has been orders by the user in the past, otherwise 0 . Technically, applying the predictive model on 374 users would have not processed accurate results. Therefore, the 
authors increased the size of the data by simulating real data. Thus, $72.7 \%$ of the data was synthesized following the distribution patterns of real data.

Weakness: However, their model did not support the influx of new data entry. The users were only allowed to buy products that pre-existed in their previous shopping baskets. The incapability of processing new product entry effects the dynamic nature of the model and hence could result in influenced predictions.

Overview: To study the user's purchase intention from online text posts, Gupta, V. et al., 2014 presented a novel feature selection technique based on text granularity and exploited social media data to evaluate their model performance. They implemented linear SVM to classify each text post into the category of PI or Non-PI (Purchase intent).

Strength: For the experiments, Gupta, V. et al., 2014 collected two types of datasets from Quora and Yahoo! Answers, respectively using a website crawler script. The former dataset included 30,000 records while the latter included 12,000 query posts. The posts collected from Quora were used for training and testing using a 10-fold cross-validation process, whereas posts from YA were used for validation purposes.

Weakness: While addressing their problem solution, they ignore the data cleaning or preprocessing task. User-generated data from online portals contain many unwanted characters such as word/alphabet repetitions are used to stress on the emotion reflected in the post (for example: "I really liiiiikkkkkeeee the product"), which might affect the model performance.

Overview: Similarly, next year Ding, X. et al., 2015 also addressed the analysis of user purchase intentions mined through social media data to provide product recommendations 
and market advertising. The authors developed a novel model technique based on a convolutional neural network to identify consumption intention by constructing a linguistic relationship between the texts.

Strength: For the experimental task, the authors collected sufficient microblog data from Sina Weibo regarding two prominent domains: Kids-baby and movie reviews. While working with big social media data, they discovered that out of 1000 labeled posts, 625 reflected the direct intention of purchase while others were showed explicit consumption purchase. They applied deep semantic analysis to detect implicit consumption intention in the text.

Weakness: The proposed transfer learning-based Consumption Intention Mining Model (CIMM), a novel framework presented by the author is directly dependent on the amount of training data provided and the length of the query. The model performance gets compromised while training minimal sentences or if the low quantity of training data is fed.

Overview: Recently, Haque, R. et al., 2019 presented their model approach to identify tweets that indicate purchase intent information. They proposed a semi-automatic technique to create a training dataset from the tweets and evaluated the trained data using recurrent neural networks. The authors compared their results achieved by the RNN model with four other baseline models: Decision trees, Random forest, logistic regression, Naïve Bayes.

Strength: The author applied deep neural networks on social media data to identify the purchase intent of twitter users. They claimed that they created an ideal training data set to 
precisely predict the intention class. For their assessment, they collected twitter data related to Food and Drink, Travel, Education \& Career, Goods \& Services, Event \& Activities, Trifle and evaluated each dataset on all five algorithms mentioned above.

Weakness: The results computed by each classifier struggled to perform for some data categories due to high-class imbalance. They achieved a high precision score as compared to recall scores for non-intent class. Concluding that their approach needs further research in terms of handling class imbalance by adopting methods like generating more data records.

Overview: In 2015, Hamroun, M. et al., 2015 introduced an interesting way to conduct intension analysis over Twitter data. For the commercial field, they presented a novel approach for enhancing semantic patterns available in the text string to identify consumption intension. The basic idea of their approach was to comprehend the ontology of the words in a tweet.

Strength: For evaluation purposes, they extracted 4 different datasets that belong to the electronic and retail banking domain from product reviewing websites. To be specific, they collected posts related to Apple iPod, Digital camera, TV, and USA bank reviews. Along with these, they used a pre-defined dataset of TREC microblog from the year 2011. The results achieved indicate that their approach of implying customer intension generated better results as compared to other previous work done.

Weakness: The proposed model in Hamroun, M. et al., 2015 extracted the patterns of customer intentions and provided consistent results on the TREC dataset which was downloaded from the web and had stable features and balanced data points, while the 
performance of the model deteriorated when applied to real-time scraped data. The data pre-processing and balancing could have leveraged the results for other datasets as well.

Overview: To skim the negative intents from the tweets, Atouati, S. et al., 2020 proposed a novel framework NeighLoR which was based on baseline algorithm Logistic regression. They created overlapping neighborhood windows of words for each tweet which helped in examining the context of each word in the string.

Strength: The authors collected two datasets that contain approximately $170 \mathrm{~K}$ tweets related to British Airways which depicts two different events. After manually labeling the dataset, they processed each tweet to find pointwise mutual information related to the words contained in the tweet. After tweaking the algorithm's cost function, they provided a detailed comparison of their result metrics with other algorithms and related works.

Weakness: Their novel approach classified the text into a negative intent class using a binary classifier. Out of $170 \mathrm{k}$, only $11 \mathrm{k}$ reflected the negative intent which resulted in highclass imbalance. Hence, they augmented two more datasets using the subsets of tweets and labeled them manually for classification, which might lead to erroneous predictions.

\subsection{Experimental Dataset Description}

Instacart (Instacart, 2020) is an online American technology company that provides on-demand single-day grocery delivery service. Instacart was founded in San Francisco in June 2012 and currently is being operated in 39 states across the United States of America today (Zaleski, O., and Huet, E., 2018). Its technology works on the crowdsourced marketplace model, for example, a User places an order and a shopper completes the 
shopping and delivers it to the user within an hour or least time possible. Grocery retailers are connected to customers by shoppers employed by Instacart through its marketplacebased model.

Through a mobile application, Consumer selects a retailer and adds all the items required from the retailer's store into a digital shopping cart. As soon as the user completes the shopping virtually, the checkout process is initiated. A shopper nearby the chosen retailer is notified to pick up the items and deliver them to the user within a short period.

On one of the official blog posts of Instacart (Instacart Online Grocery Shopping Dataset, 2017), the company released a public dataset as an open-source under Terms and conditions. The dataset is basically a relational set of files that contains containing information on 3 million grocery orders from more than 200,000 de-identified users from 2017 (January 1 to December 31).

\subsubsection{Confidentiality aspect of the dataset}

Instacart has taken necessary measures to protect the privacy of the users and retail partners and to safeguard that the data is entirely anonymous (Instacart Privacy policy, 2017):

1. The only information that describes the activity of a user is their sequence of orders and the products in those orders.

2. All the unique IDs mentioned in the dataset cannot be linked back to any other ID as they are chosen randomly to maintain discretion.

3. Only those products are included in the dataset which is ordered by multiple users from multiple retailers and retailer ID is kept private. 


\subsection{Social media Dataset}

Social media platforms are usually used by people to express their desires, share their experiences and opinions (Pak, A., \& Paroubek, P., 2010). Since the increasing popularity of social media, we will leverage our experimental data at a scale to support our research study that has never been previously achieved in related work. Social media analysis is a study of people's views and communications shared publicly on the broadcasting platform. Recently the use of social media platforms such as Facebook (Facebook website, 2020), Twitter (Twitter website, 2020) has been evolved in the world of data science, so, many procedures surrounding the application of social media can be used to triangulate the data source used in our experiment (Makice, K., 2009; Beautiful Soup, 2020 ). Such data provides us with the opportunity to mine the user activities to identify users' consumption intention from user-generated content so that improved tailored goods can be recommended. In this section, we investigate the social media data of Instacart users to summarize the intentions of the users and integrate the useful knowledge with our tentative dataset.

To investigate the efficacy of lexical features for analyzing the sentiments of users, we crawled the tweets based on "Instacart" as a keyword from January $1^{\text {st }}, 2017$ to December $31^{\text {st }}$, 2017 via the Twitter API (Makice, K., 2009). We used Python 3 library Textblob for processing the textual data of the microblogging website. It provides a simple API to perform natural language processing (NLP) to determine the emotional content of a word. We created a script based on an article of sentiment analysis on the website GeeksforGeeks (Twitter sentiment analysis using Python, 2020), which automatically 
collects the tweets using Twitter API and assigns the polarity of a review based on the average semantic orientation extracted from the TextBlob package.

The data set contains 1300 records and 2 fields i.e. Text and polarity value. The attitude of the text data is scored using polarity values that range from 1 to -1 . The value that is closer to 1 indicates positive sentiments while values closer to -1 indicate negative sentiments. Polarity values are then plotted in a graph to visually understand the overall sentiments of Instacart users.

Figure 1. Sentiment Polarity of Instacart Tweets

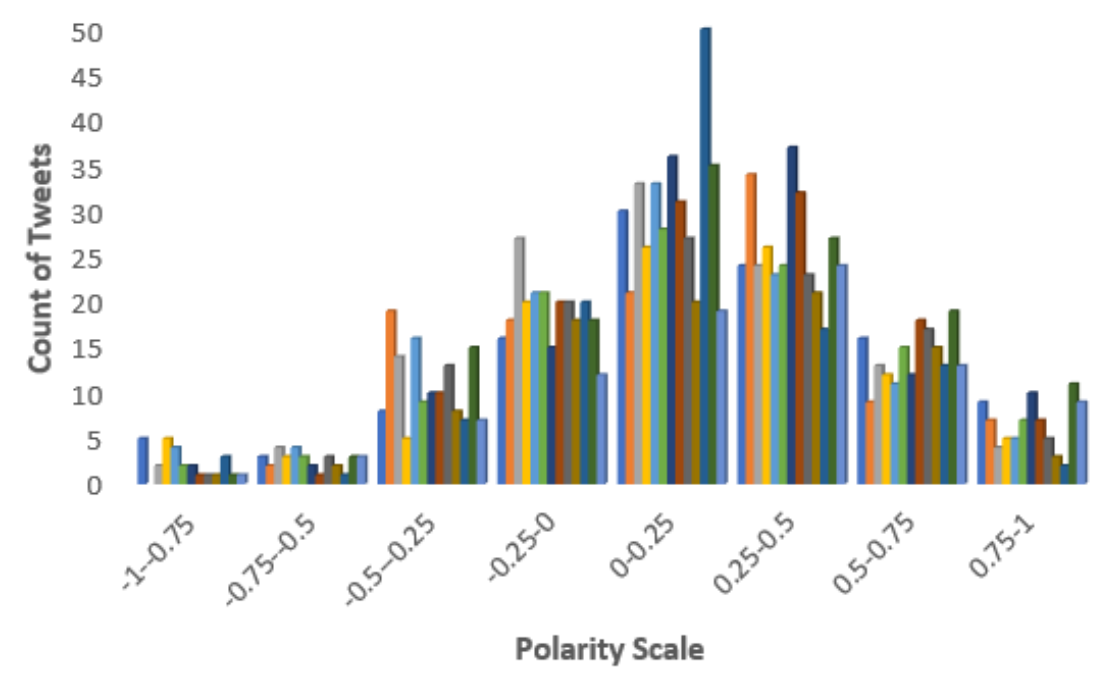

Figure 1 displays a histogram of polarity values for tweets on Instacart services. The $x$-axis depicts the polarity scale bins of size 0.25 each, and the y-axis represents the number of tweets. The color of bars in the plot differentiates the number of tweets at a specific polarity score.

For this plot, we removed the polarity values that equal to zero to better highlight the distribution. Based on the data collected, the average polarity of sentiments is 0.13 . Therefore, we can perceive that most of the tweets based on Instacart were relatively neutral. 
Figure 2. Number of tweets in the year 2017

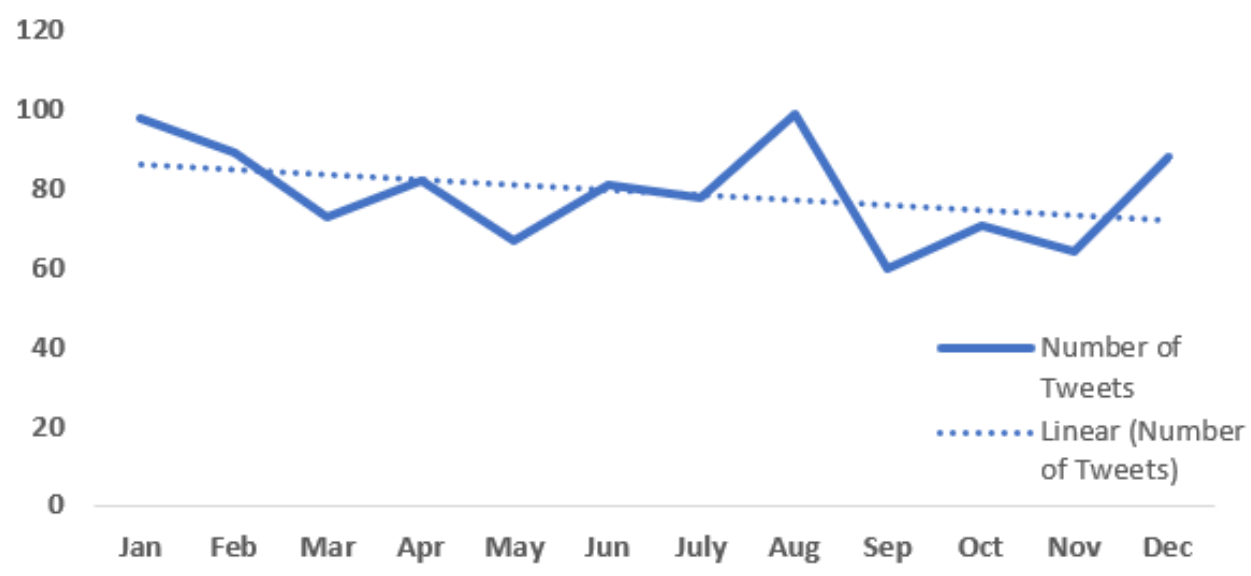

To understand the collected data's pattern, figure 2 represents the trend of Instacart tweets over the year 2017. The number of tweets peaked in August, but the overall trend kept on decreasing by a few numbers every month. We also tried to represent the text data of tweets visually in the form of a word cloud using an online tool (Tweetcloud website, 2010). The most frequent words such as Instacart, delivered groceries, thanksgiving, love, stand out in larger and bolder fonts in Figure 3.

Figure 3. Word Cloud of Tweets

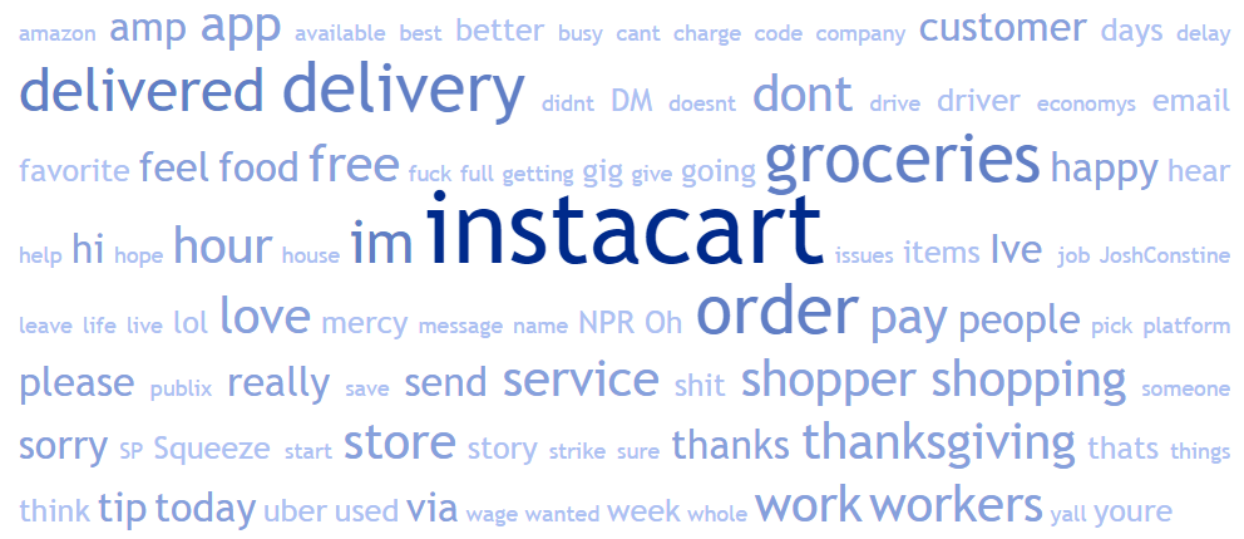

By scrutinizing the consumer's desires, thoughts, preferences, and frustrations mentioned online, we can say that Instacart received an insignificant response and market 
popularity, and hence, the objective of this research work is to comprehend customers' desire to achieve more positive polarisation in future.

\subsection{Experimental Data Dictionary}

The dataset provided by the Instacart website (Instacart website, 2020) is for noncommercial use and is explicitly provided in form of a relational set of files describing customers' orders over time to extract a better understanding of both the characteristics of its products and the relation with the orders. To maintain the confidentiality of their consumers and retailers, the only information provided about users is their sequence of orders and the products in those orders. Similarly, for retailers, Instacart's retailer subset is highly biased in terms of production data. All the user's IDs and retailer IDs are randomized and cannot be linked back to any other ID.

The dataset contains a record of over 3 million grocery transactions from more than 200,000 anonymized Instacart users from North America. which is dispersed in five CSV files: orders, products, order_products, aisles, departments. Each entity (such as customer, product, order, aisle) has a correlated unique id also known as the primary key. The files and variable names contained in the dataset are mostly self-descriptive. The data structure of each table can be represented in the form of a SQL database diagram:

Figure 4. ER diagram for the Instacart data tables 


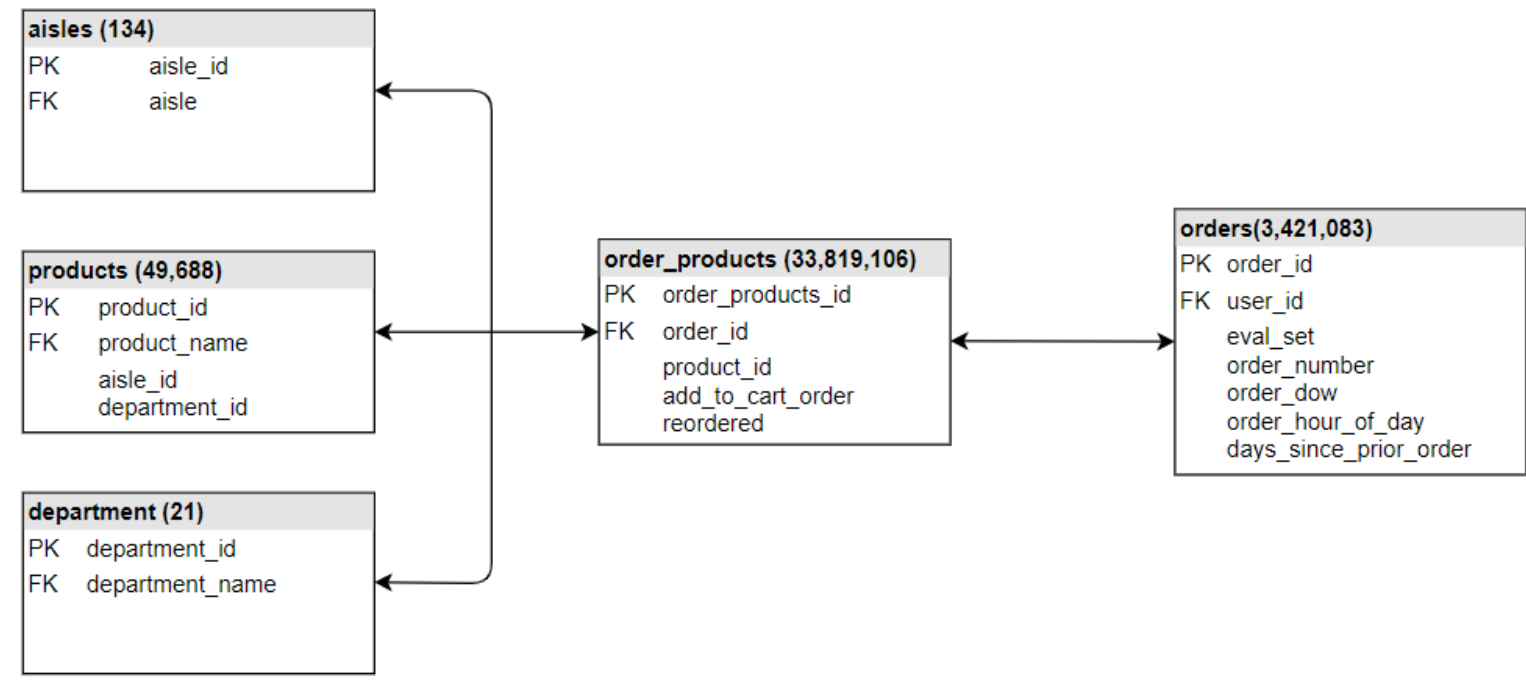

- "orders.csv":

This file consists of 3.4 million rows of orders that are placed by 206 thousand distinct users. It provides a list of all orders we have in the dataset, but it does not reveal which product was ordered by the specific user. The order_id field in the data is considered as the primary key for differentiating each row.

For the orders data file, we list the description of each field in the table below.

Table 1. Basic information about "orders" dataset

\begin{tabular}{|l|l|l|}
\hline \multicolumn{1}{|c|}{ Field } & \multicolumn{1}{c|}{ Datatype } & \multicolumn{1}{c|}{ Description } \\
\hline order_id & Integer & The identifier of the order \\
\hline user_id & Integer & The identifier of the user \\
\hline eval_set & String & The category of the evaluation set for the order (prior, train or test) \\
\hline order_number & Integer & $\begin{array}{l}\text { The sequence number of the order placed by the user (1 }=\text { first, } \mathrm{n}= \\
\text { nth) }\end{array}$ \\
\hline order_dow & Integer & The day of the week when the order was placed \\
\hline order_hour_of_day & Integer & The hour of the day when the order was placed \\
\hline days_since_prior & Integer & The days before the last order was placed, capped at 30 \\
\hline
\end{tabular}

\section{- "products.csv":}


This file contains 49,688 records that describe the names of the products with their corresponding product_id as the primary key of association. Additionally, the aisle and department IDs of the product are included.

Below, we list the description of each field in the table for product data.

Table 2. Basic information about "products" dataset

\begin{tabular}{|l|l|l|}
\hline \multicolumn{1}{|c|}{ Field } & \multicolumn{1}{c|}{ Datatype } & \multicolumn{1}{c|}{ Description } \\
\hline product_id & Integer & The identifier of the product \\
\hline product_name & String & Name of the product corresponding to its ID \\
\hline aisle_id & Integer & The identifier of the aisle \\
\hline department_id & Integer & The identifier of the department the product belongs to \\
\hline
\end{tabular}

- “order_products.csv":

This file provides information about products (product_id) that were ordered with order_id as the primary key to distinguish each record. It also contains the information about the sequence of putting the product in cart (add_to_cart_order). It also delivers the knowledge of whether the product was repurchased (1) or not (0).

Table 3. Basic information about "order_product" dataset

\begin{tabular}{|l|l|l|}
\hline \multicolumn{1}{|c|}{ Field } & \multicolumn{1}{c|}{ Datatype } & \multicolumn{1}{c|}{ Description } \\
\hline order_id & Integer & The identifier of the order \\
\hline product_id & Integer & The identifier of the product \\
\hline add_to_cart_order & Integer & The order in which each product was added to cart \\
\hline reordered & Integer & 1 if the product was repurchased, 0 otherwise \\
\hline
\end{tabular}

The order_products table consists of the latest cart ordered by users and thus containing the evidence about whether the product was repurchased in the most recent cart. This signifies our target variable.

- "aisles.csv": 
The information of 134 distinct aisles is contained in this file. Each aisle is distinguished with aisle_id and the name of the aisle.

Table 4. Basic information of "aisles" dataset

\begin{tabular}{|l|l|l|}
\hline \multicolumn{1}{|c|}{ Field } & \multicolumn{1}{c|}{ Datatype } & \multicolumn{1}{c|}{ Description } \\
\hline aisle_id & Integer & The identifier of the aisle \\
\hline aisle & String & The name of the aisle \\
\hline
\end{tabular}

\section{- “departments.csv":}

This file contains the information of 21 distinctive departments. Each department is linked with unique department_id and its name.

Table 5. Basic information about "department" dataset

\begin{tabular}{|l|l|l|}
\hline \multicolumn{1}{|c|}{ Field } & \multicolumn{1}{|c|}{ Datatype } & \multicolumn{1}{c|}{ Description } \\
\hline department_id & Integer & The identifier of the department \\
\hline department & String & The name of the department \\
\hline
\end{tabular}

\subsection{Data Visualization}

In order to investigate the insights of the data and to inform feature engineering, we familiarize ourselves with the data by exploring the information in the form of visual graphs and plots. We used python programming language on an open-source web application jupyter notebook 6.0.2 (Jupyter notebook, 2020) for the visualization of data which allowed us to do descriptive readable Analysis, Diagnostic analysis, and predictive analysis. Exploratory analysis of our dataset will help us to identify customer's behavior patterns through their purchase activities with the aim of find similarities and differences.

Since the Instacart data is subdivided into multiple relational sets, the research opportunity is abundant. Each table has been explored in terms of visualization to extract some surface-level insights about the dataset. To follow the structure of the data 
visualization section, initially, the products table is visually investigated in terms of purchased items by the users. Following that, the analysis of the orders table is performed in order to comprehend the customer's shopping behavior. To conclude, the department and aisles dataset is evaluated to infer the maximum revenue-generating items.

To address the pattern of the number of items purchased by the users in a single order, we investigated the order product data frame. It is evaluated based on the sequence order in which the item is added to the cart.

Figure 5. The number of products purchased per order

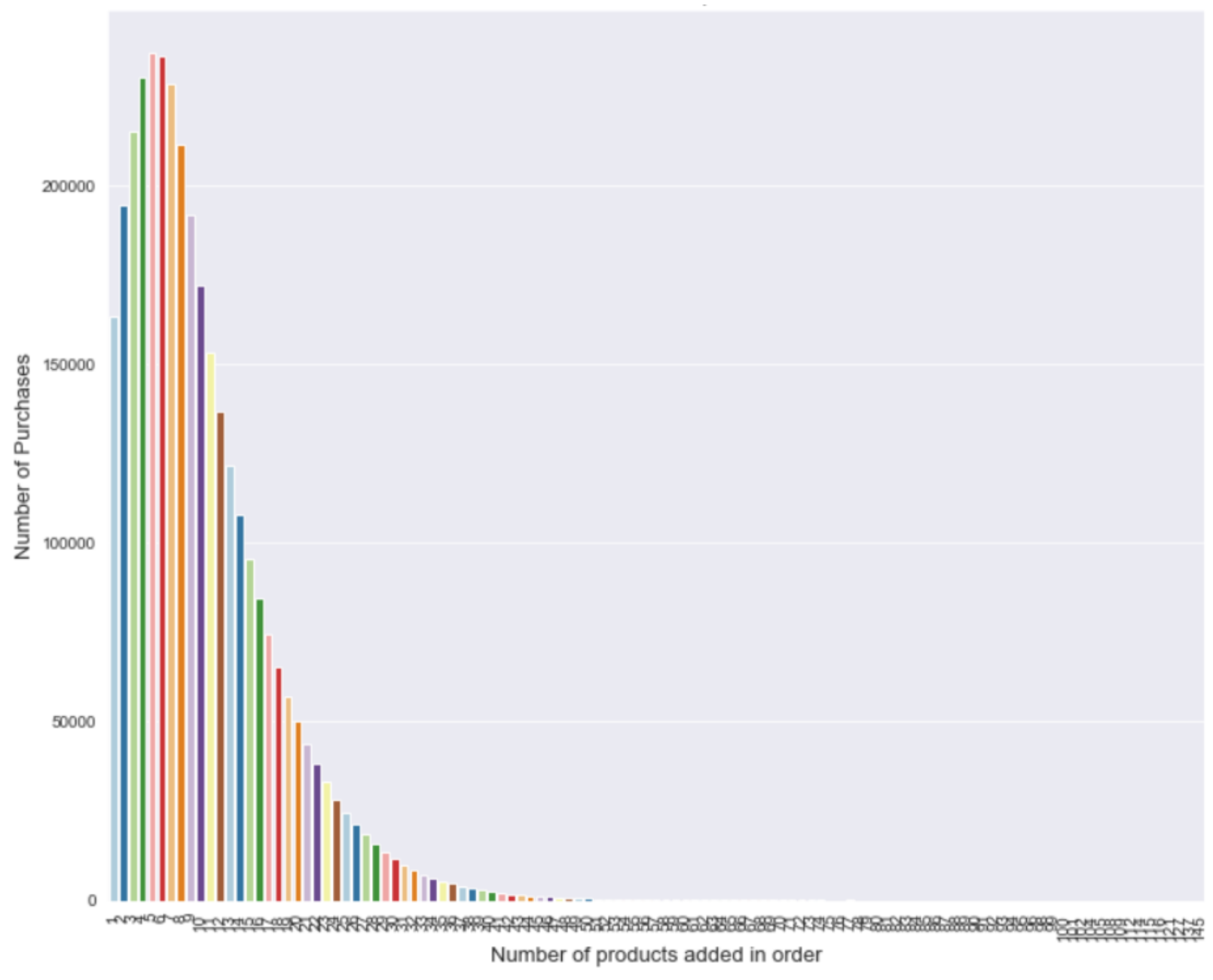


We can observe in Figure 5 that people are more likely to buy 1 to 10 products in a single purchase which maximizes at 5 items per order. Very few users buy 30 products or more. Only the trivial number of orders contains more than 50 items to purchase.

To get the insight of top products that are purchased by the users, we merged orders and product data frames and extracted the top 10 items. By plotting the most re-purchased items against the number of orders placed, we can conclude from Figure 6 that the most selling products are Bananas $(491,291)$ followed by Bag of Organic Bananas $(394,930)$ and Organic Strawberries $(275,577)$.

Intriguingly, 11 out of 15 most frequently sold products include the word "Organic" in their names. Hence, as visually predicted from the data, we can conclude that organic products have a high possibility of selling as compared to non-organic items.

Figure 6. Top selling products

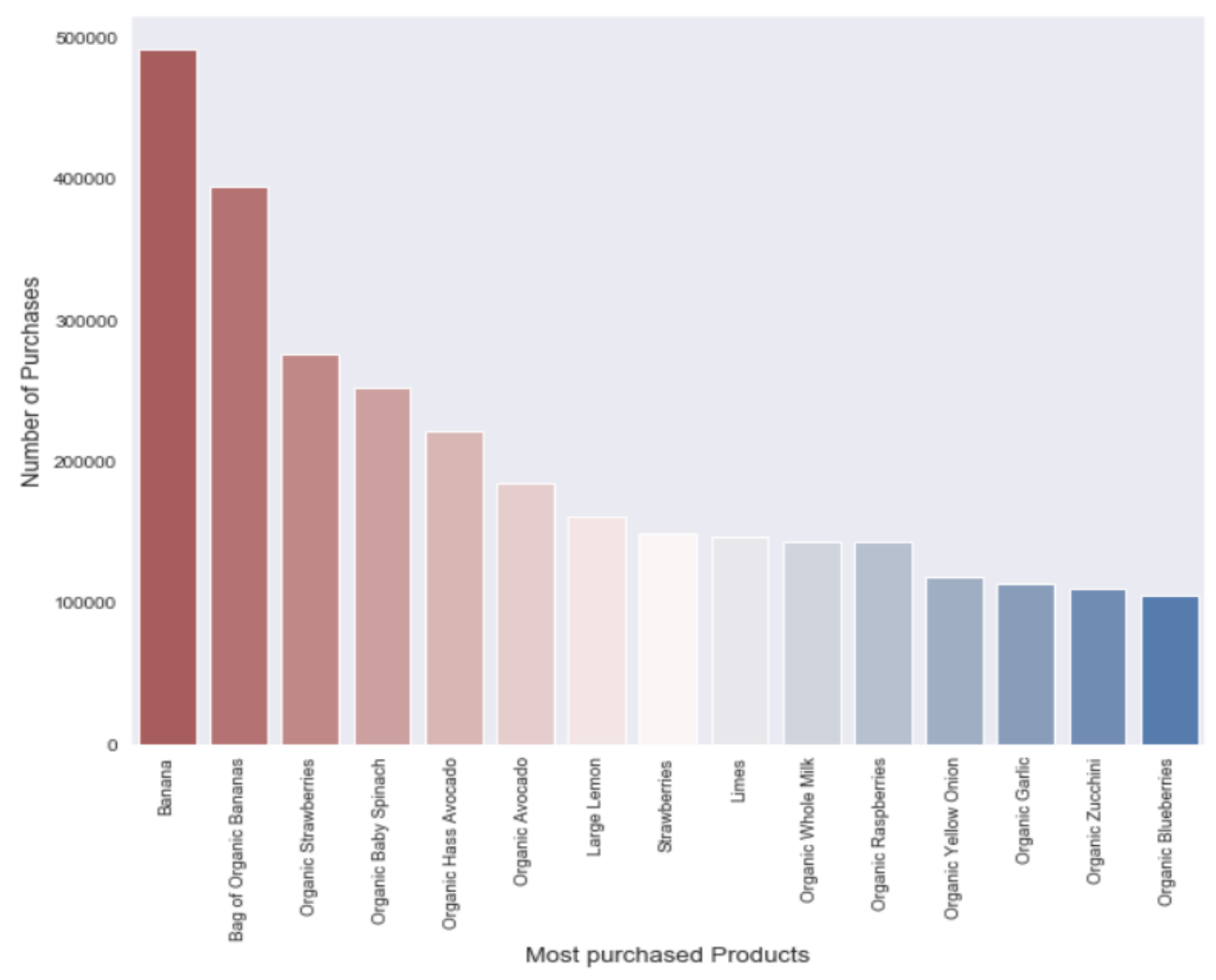


To determine the extent of repurchased items against the total products, we evaluated the ratio of repurchased products and the total number of items. The plot below (Figure 7) indicates the products that are usually most repurchased by probability. The five topmost re-ordered items, by probability, are Serenity Ultimate Extrema Overnight Pads (0.933), Chocolate Love Bar (0.922), Maca Buttercups (0.894), Benchbreak Chardonnay (0.892), and Organic Blueberry B Mega (0.889).

Figure 7. Products with the maximum probability of repurchase

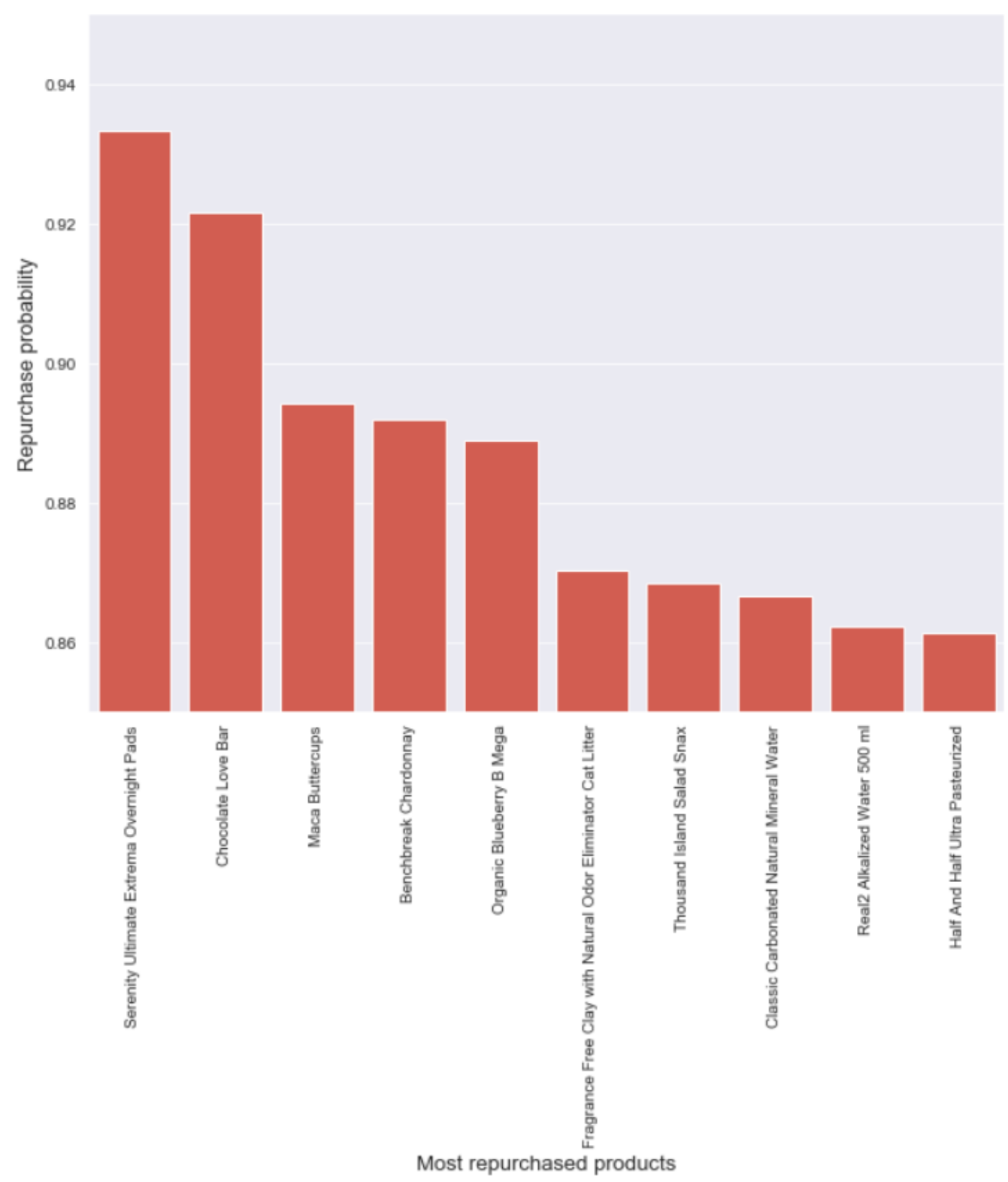


Overall, there are 3,346,083 unique orders for 49,685 distinctive products with 206,209 exclusive customers in the whole dataset. To recognize the patterns orders placed by consumers, we assessed the orders data frame to obtain visual expertise in Figure 8.

The number of orders placed by each unique customer is evaluated by calculating the order count against each $u s e r \_i d$. From the bar graph plotted below, we can observe that most customers placed about 4 orders with an outlier 100 orders placed by approximately two thousand users.

Figure 8. Number of orders per customer

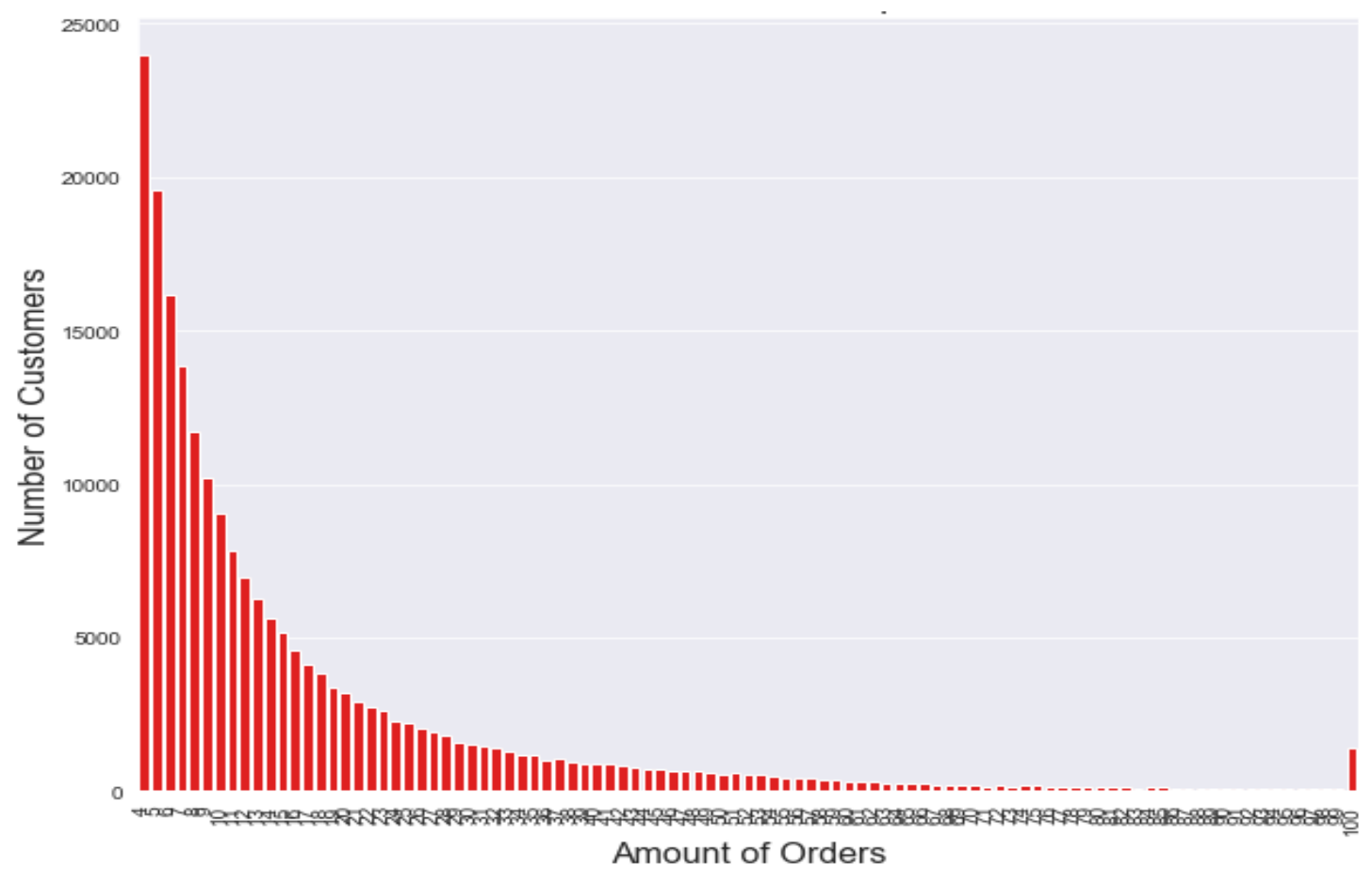

Following, we merged products dataset with departments, to look at the most important department categorized by the number of products sold. As shown in Figure 9, the five topmost departments in terms of selling products are Personal Care $(6,563)$, Snacks 
$(6,264)$, Pantry $(5,371)$, Beverages $(4,365)$, and Frozen $(4,007)$. The minimum number of products are bought by users from the bulk department.

Figure 9. Number of products purchased per department

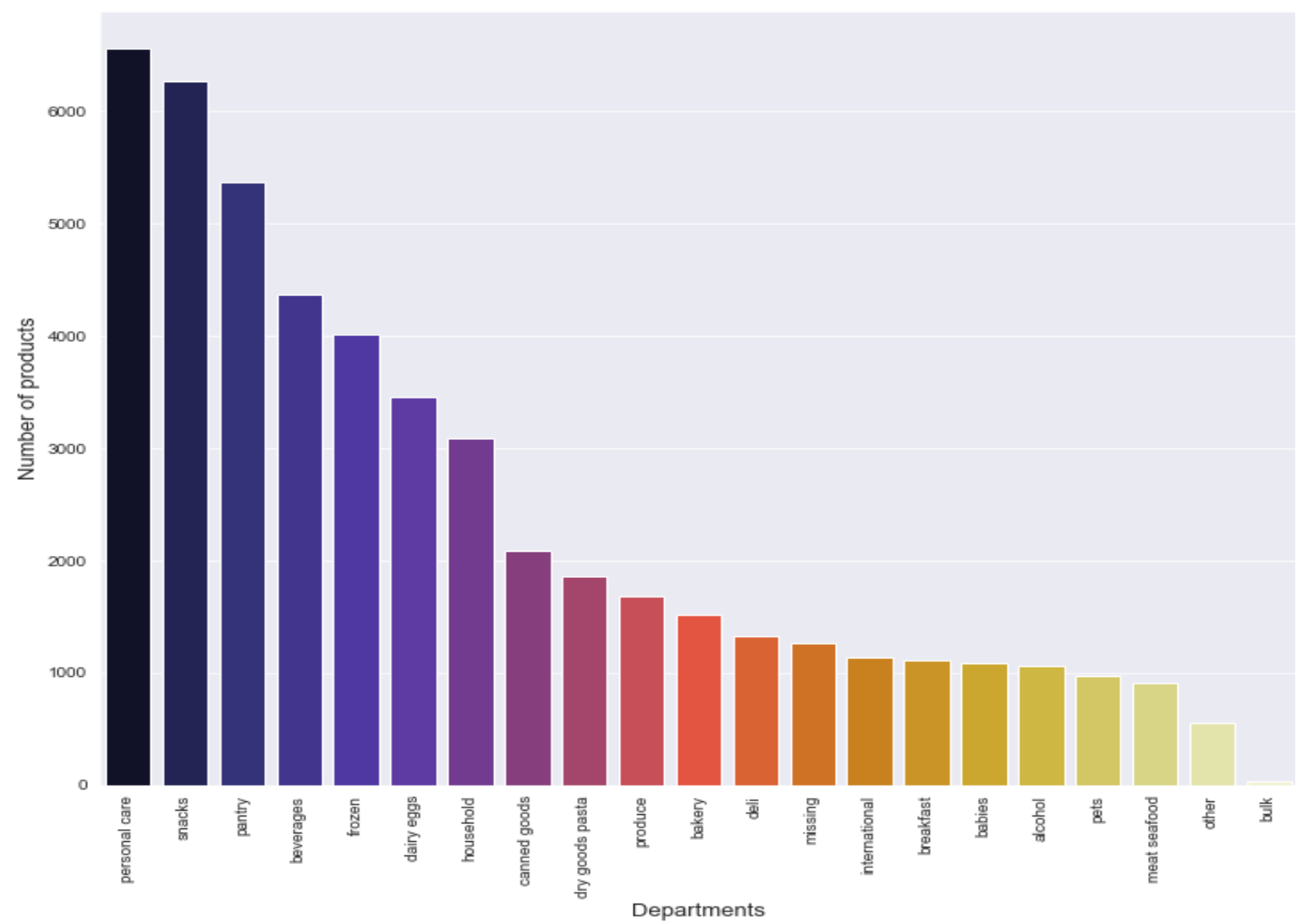

To plot the rush hours of the Instacart shoppers, we counted the orders placed per hour with respect to the order_id to obtain a unique order count of each department. The line graph below represents that during a regular day, customers mostly placed their orders between $8 \mathrm{AM}$ and $7 \mathrm{PM}$, and the highest proportion of purchases are done from produce followed by dairy egg departments.

Figure 10 provides an aggregate view of when items from each department are purchased, which may conceal some of the underlying composition. We could say that it is best to shop before $10 \mathrm{AM}$ or after $3 \mathrm{PM}$ to beat the customer traffic. There are some 
departments where the number of products purchased is uniform throughout the day such as alcohol, which is closer to the $\mathrm{x}$-axis. However, there are some items from the departments that do not have any defined pattern of purchase. To conclude we can say that not all customers shop from each department with the same frequency.

Figure 10. Average footfall per hour in a day
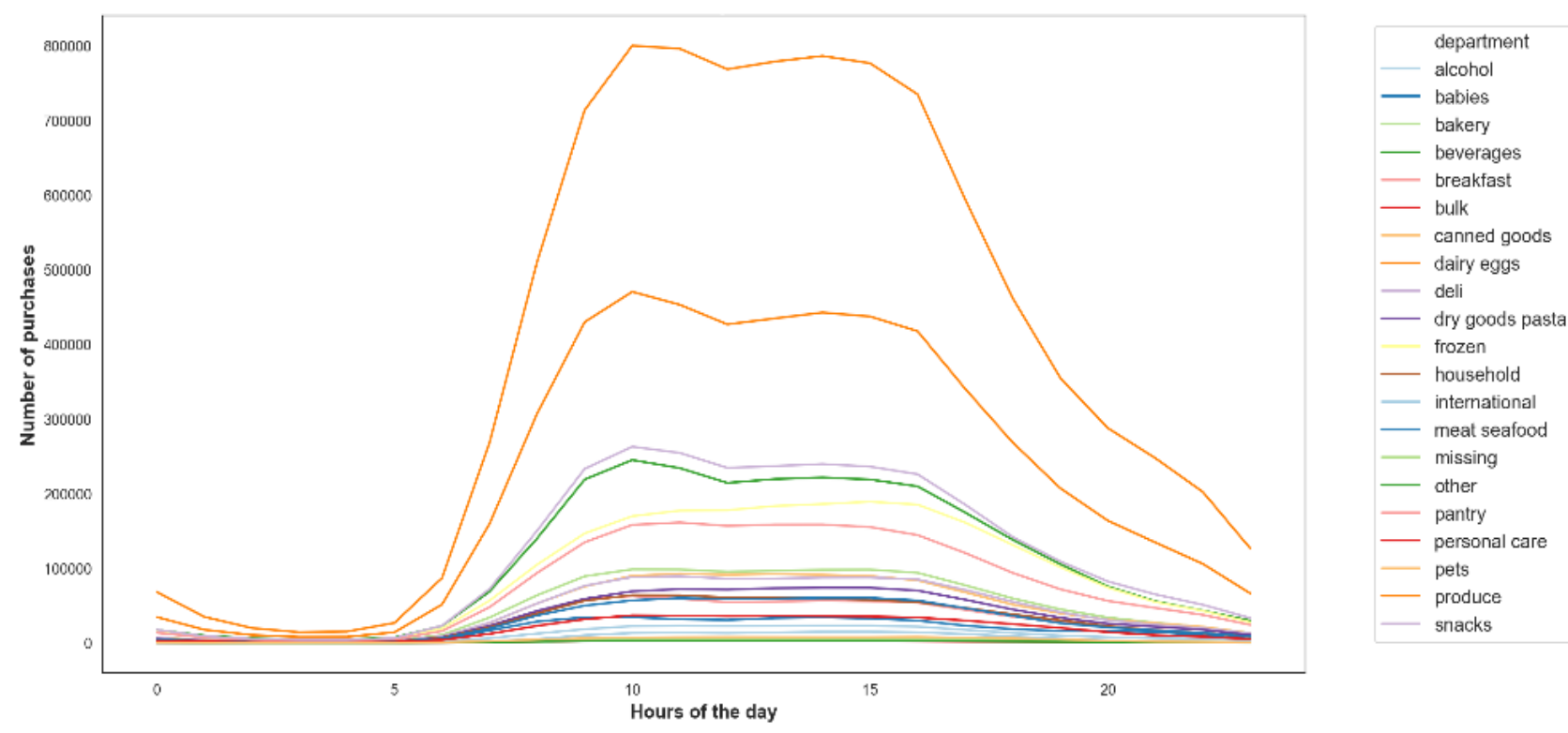

Similarly, to plot the traffic per day in a week, we observed the order_dow feature in orders dataset in correlation with the products data frame. The lines are plotted according to the purchase rate of each department individually. From the graph below, it appears like users primarily placed their orders during the weekend ( 0 is Saturday and 1 is Sunday) which further points to the fact that most the people shop either during the start of the week or at the end. This line graph (Figure 11) estimates the frequency of items sold per day. Few departments are consistent in selling each day of the week such as pets, meat seafood (as they are closer to the $\mathrm{x}$-axis). However, the produce department followed by the dairy department can be termed as the best seller of each day with a significant amount of 
increase in weekends. In nutshell, selling frequency per week varies significantly for each department.

Figure 11. Average footfall per day in a week
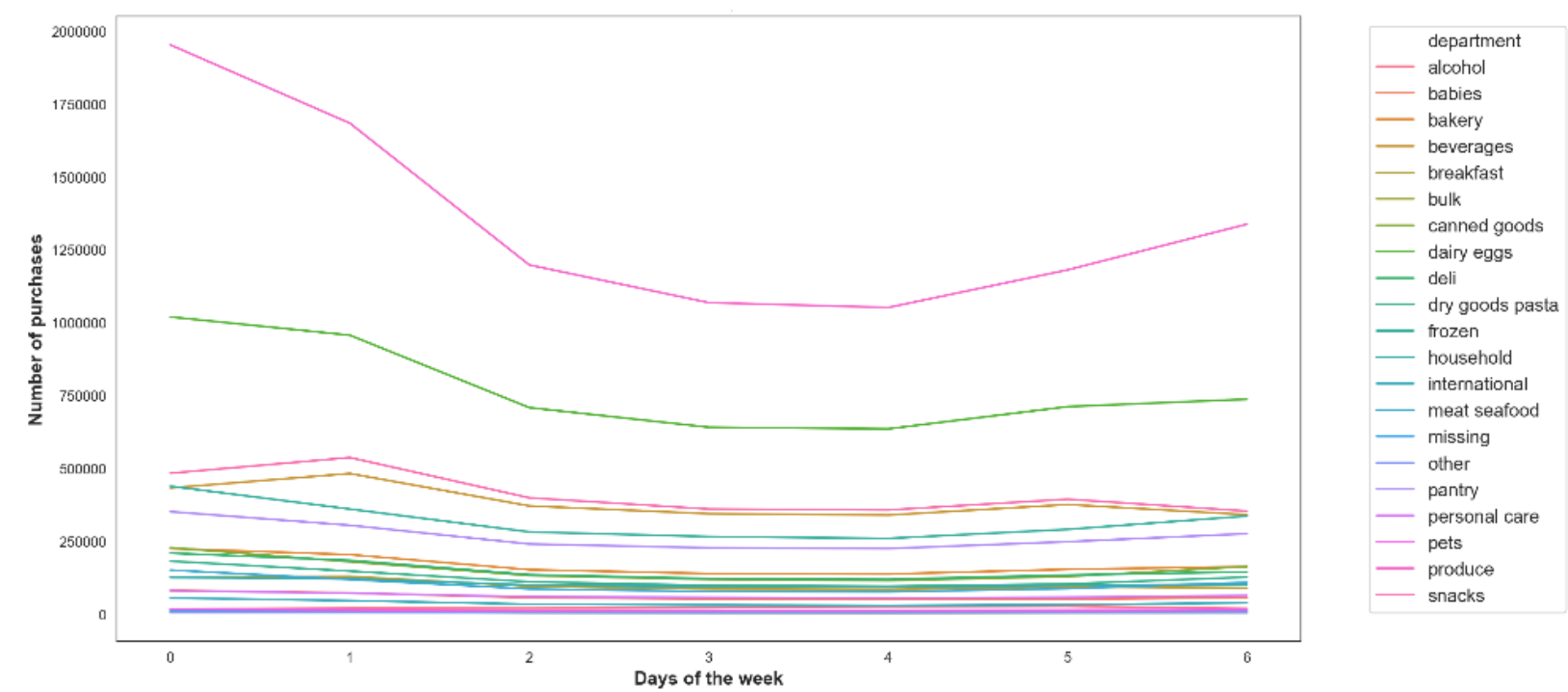

To get the vision of most prominently selling aisles, we merged the order table with aisles in correlation to the total number of orders placed. The bar graph below depicts the top 15 aisles from where the highest number of items are purchased. The maximum number of purchases is of fresh vegetables $(150,609)$ followed by fresh fruits $(150,473)$ and packed produce items $(78,493)$ as shown in Figure 12. 
Figure 12. Topmost selling aisles

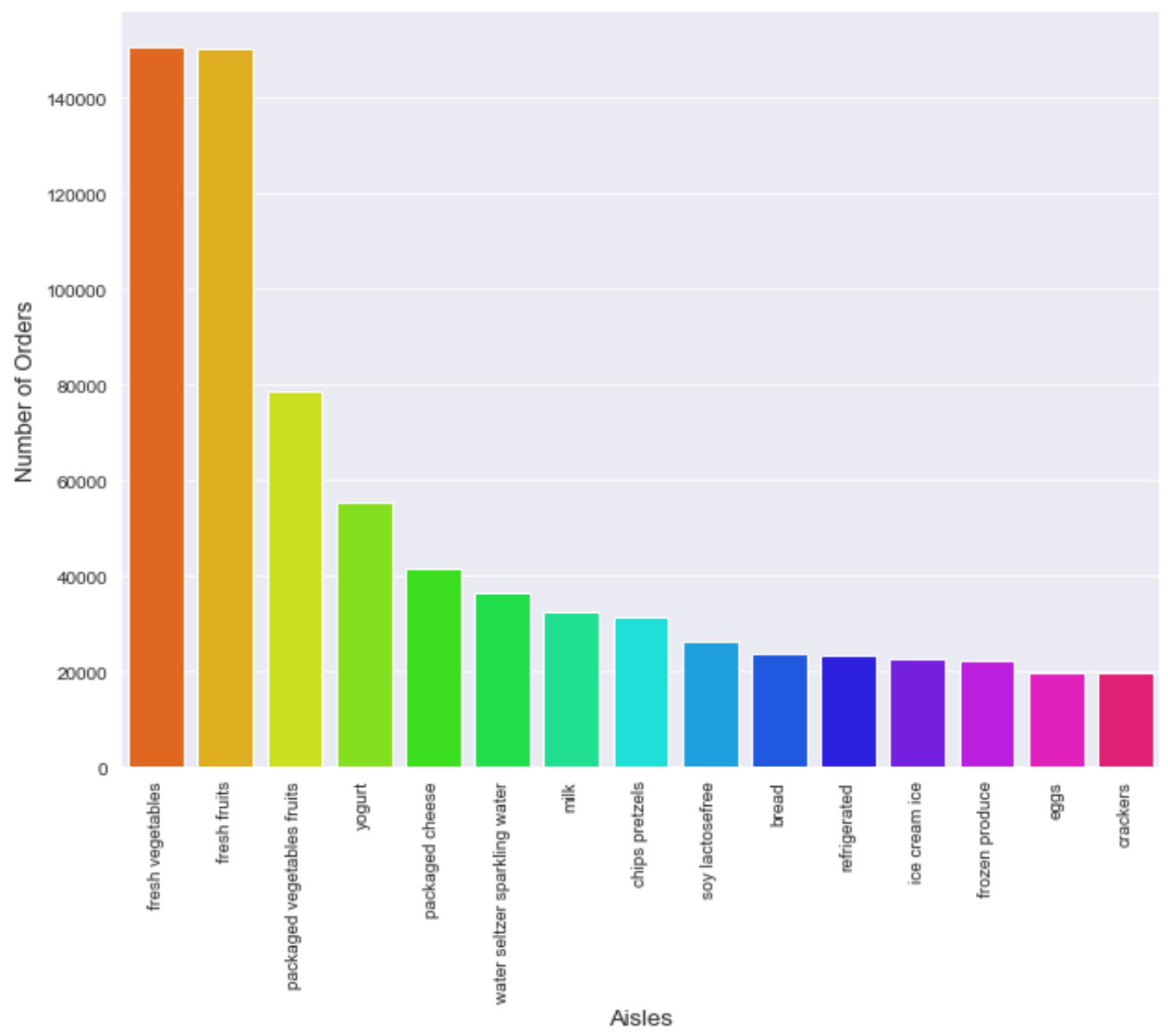

Another interesting pattern is evaluated that corresponds to the number of days between two orders by a user. The feature days_since_prior from the order data frame is plotted against the order count. As we can see in Figure 13, the highest number of orders is spaced apart by 30 days followed by 7,14 and 21 days. This means most of the users repurchase their groceries monthly, weekly, and biweekly. 


\section{Figure 13. Period of repurchase}

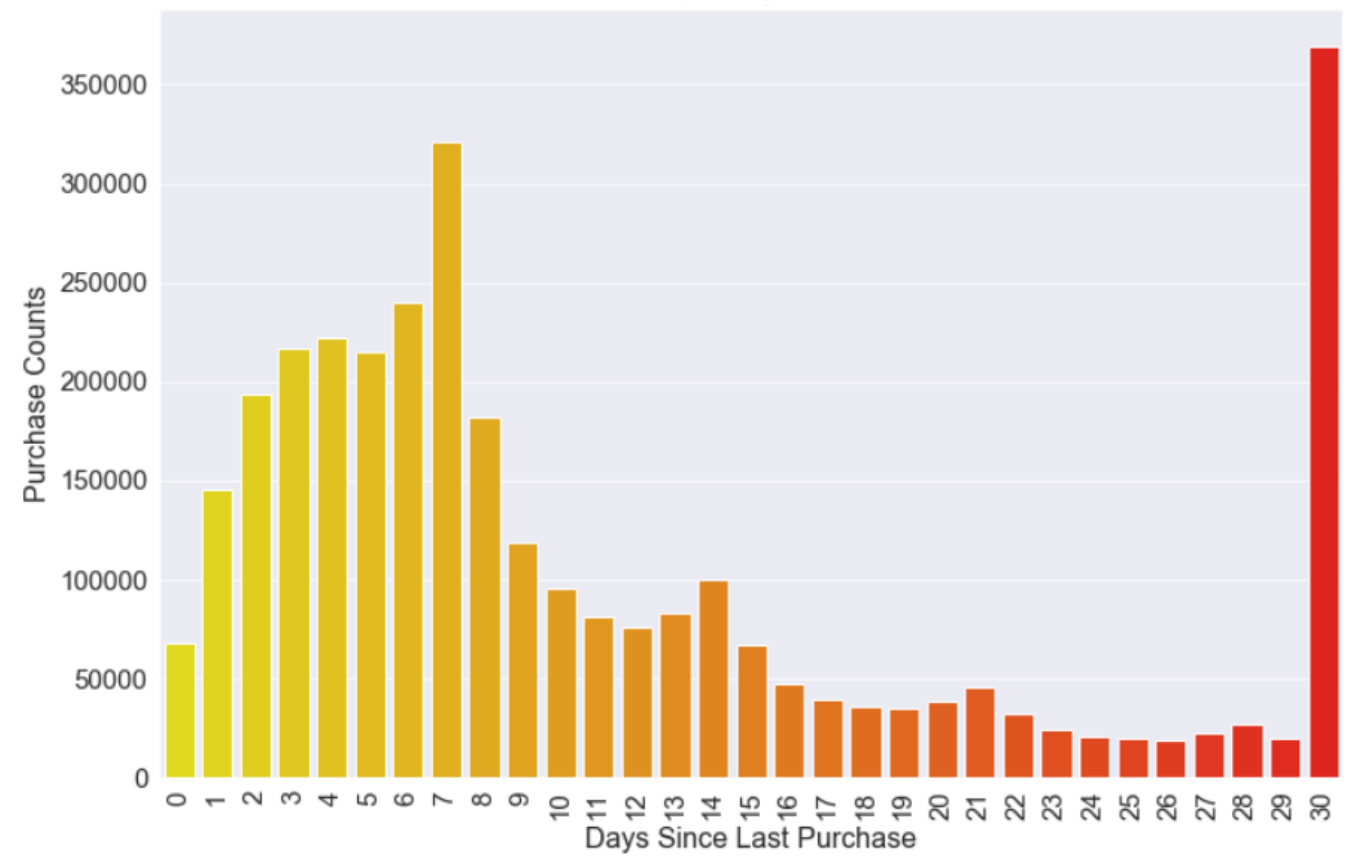

To conclude, this visual exploratory analysis of the data provided allows us to comprehend the fine-grained details about the customer's grocery shopping patterns on the Instacart platform. Information like most frequently purchased items, rush hours of the customers, the period between two orders for a unique customer could be considered as a preliminary step of our research. This visualization could also help retailers in managing the inventory at the right time for the right number of users. 


\section{Chapter 3: Review of Existing works}

In this chapter, we introduce the technical features of existing work in this research field. The literature evaluation is done in two segments. The first segment is in chapter 2 , which delivers the backbone to our dataset by providing instructive knowledge derived from the dataset used in previous works. This chapter is the second segment which reviews the technical domain of the study. We first briefly introduce the recommendations-based research on embedding systems, one of the most successful techniques for recommender systems, which includes the research work on memory-based methods as well as modelbased methods. Then, we make a comprehensive survey about the machine learning algorithms along with association rule mining used in the field of recommendation. We also present the literature of some state-of-art techniques used in the range of recommending groceries. At last, we deliver a comprehensive comparative analysis of the listed literature.

\subsection{Technical relation}

Many literature analyses provide a substantial reason to unfold the relationship between customers and retailers (Deloitte Touche Tohmatsu Limited, 2003). As mentioned in Deloitte Touche Tohmatsu Limited, 2003, Nowadays, many tools and theories describe how to manage customer relationships efficiently and strategically which is being used by modern businesses and organizations (Le, D. T. et al., 2017; XingFen, W. et al., 2018; Sakar, C. O. et al., 2019; Haque, R. et al., 2019). All these methods are developed by reviewing areas oscillating from consumer psychology and behavior on the customer at an 
individual level. To provide direction to our research study, we will explain various stateof-art prediction models that are being used so far.

\subsubsection{Embedding-based Recommendations}

Embeddings are a method to represent categorical value in continuous vector form. Many research studies have been done in the field of natural language processing embedding based models, such as word analogies (Grbovic, M. et al., 2015; Vasile, F. et al., 2016).

Overview: Wölbitsch, M. et al., 2019 focused on extending the state-of-art embedded based recommendation framework for in-store retail commerce. They aimed to process bundle purchase information without having to maintain separate models for different geographical locations of retail stores. The information collected from Radiofrequency Identification based data streams provides a strong foundation for their research.

Strength: The strength of their research study was that it has provided a unified solution using data augmentation with a point of scale information combination which tends to leverage the robust and accuracy in the recommendation. Natural language processing embedding based models are highly in demand where words represent low dimensional vectors (Li, Y. et al., 2015; Pennington, J. et al., 2014).

Weakness: While evaluating their approach, they considered only a single dataset of realworld fashion retail stores, which might have restricted them to explore their method out of the domain. Their model suffered because of a co-purchase recall match. In nutshell, they could enhance their performance by considering metadata information like past revenue-generating items, size of the store. 
Overview: In the field of recommender systems, Barkan, O., \& Koenigstein, N., 2016 mapped item-based collaborative filtering under Skip-gram with Negative Sampling approach (SGNS) (Mikolov, T. et al., 2013), that is word2vec to figure the products that could be promoted in conjunction with the item of interest. A modified version, item $2 \mathrm{vec}$ approach which was a neural embedded algorithm, produced the similarities between the items based on SGNS based on unsupervised learning from a sequence of entities. This approach was considered as a flavor of embedding scheme in which neural network weights use backpropagation to generate correlations of products.

Strength: While comparing with item-item singular value decomposition (SVD), their approach outperformed as it provided higher resolution resemblances within the item categories. Moreover, the item2vec approach provided better recommendations than SVD even when the store data information was sparse in terms of qualitative evaluation.

Weakness: Barkan, O., \& Koenigstein, N., 2016 used embeddings to model items in a low dimensional space to calculate a similarity metric, however, they neglected the temporal ordering of the items in the basket for purchase.

Overview: Grbovic, M. et al., 2015 exploited the word2vec algorithm and modified it as prod2vec to generate product recommendations based on e-mail recipes received by Yahoo Mail users. They proposed an extension of the neural language-based algorithm that embedded user information to generate product recommendations personalized according to them.

Strength: The proposed Prod2Vec system of recommendation took the purchase receipts from emails to present recommendation candidates. The main advantage of their proposed 
the solution was that they considered the user's recent behavioral patterns in providing personalized recommendations.

Weakness: However, the approach in Grbovic, M. et al., 2015 neglected the time decay factor related to older emails which may induce an error in recommendations. Moreover, this framework was only capable of computing short term interests shown by the customers and neglected the user-related past purchasing history.

Overview: Vasile, F. et al., 2016 introduced an extended version of the prod2vec algorithm (Grbovic, M. et al., 2015) by integrating additional metadata to compute item similarities on an open music dataset. They combined the word embedding method to matrix factorization to analyze the relative value of side information. Features like users and items were embedded in the same latent space.

Strength: The proposed technique influences the former user interactions with items to compute the low- dimensional embeddings. Introducing the model with additional metadata information leveraged the performance of item embeddings. Although, they exploited the content feature assuming features interact linearly, which intrudes as a drawback of overspecialization.

Weakness: After comparing the experimental results of Meta-Prod2Vec with Item2Vec, it has been proven by Vasile, F. et al., 2016 that the former approach always outperforms the latter in providing recommendations. However, both approaches failed to suggest personalized items to users. Item embedded techniques only train the model based on the representation of items and neglect the user representation. Hence, there comes a requirement of an approach that will consider both item representation as well as user interactions in providing recommendation suggestion. 
Overview: In 2018, Grbovic, M., \& Cheng, H., 2018 addressed the problem of recommending a real-time personalized short-term rental marketplace, known as Airbnb, according to both hosts as well as guest preferences. They conducted a study based on listing and user embedding techniques along with concepts like global context and explicit negative signals to learn the low-dimensional representation of home listing.

Strength: One of the prominent impacts of their recommendation was, while training, they encoded global context and preference signals to reduce the rejections. Their embedding system surpassed the click-through rate by $20 \%$ as compared to other related works done in similar listing recommendations.

Weakness: However, for leveraging such pre-trained item embeddings for user preference, the proposed modeling technique had not been extensively studied. They observed some drawbacks in traditional negative samplings. Hence, they swapped negative sampling probability with a set of probabilities suited for different subsets of embedding objects.

Overview: In the year 2016, Chen, T. et al., 2017 worked on providing recommendations based on textual data. They introduced a novel framework of join text embedding by exploiting user and product interactions. The model results of Chen, T. et al., 2017 proved that their approach phenomenal results for new text recommendations.

Strength: Chen, T. et al., 2017 used a text embedding approach that learned end-to-end to provide personalized recommendations to users based on user interaction with items. They further extended the area by introducing a joint text embedding model that leverages the textual data with a limited amount of user interaction. This approach mingled the supervised text embedded approach and unsupervised joint text embedding. 
Weakness: The downside of their research work was the filtering mechanism which provided a recommendation that can be anticipated in the user's vision. They require to extend their research area in a way that recommendations provided by the systems should be unique and unpredictable by users.

\subsubsection{Recommender Systems based on transactional data}

Recommender systems are a hot topic in the area of providing customized recommendations to consumers. These decision tools allow many firms to analyze customer purchase behavior and history to address the problem of the next purchase suggestion.

Overview: Recently, Mohan, A. et al., 2019 introduced a recommendation system that can be implemented within a business intelligence platform known as SmartEmma, where no previous interactions of users are defined. Their system worked on item-item collaborative filtering in the field of information retrieval and recommendation.

Strength: In their study, they presented an evaluation grocery retailing application, a digital platform for retailers with small scale businesses. The application allowed retailers to distinguish the items that were high in demand amongst consumers and provided a userfriendly e-commerce interface.

Weakness: While evaluating the recommender system on the user base, transactional data of users was created based on the number of items purchased in one order and individual item's rating. Due to smaller Figures of data, the score of similarity suffered for $95 \%$ of the users. Hence, extensive testing tools are required to provide the base to the application. Overview: In the meantime, Kouki, P. et al., 2019 addressed the problem of bundle recommendation of products that shared some common attributes. They proposed a 
recommendation algorithm that generated a set of candidate's product collection by combining product hierarchies and transactional data of co-purchase information.

Strength: In their paper, to target the recommendation problem, they proposed an algorithm that produced a set of items that share the same theme by exploiting co-purchase information. The domain knowledge of each purchase was used to learn similarities in order to generate a collection of items and their attributes.

Weakness: They manually collected relationship using domain experts and compared it with automated relationship solutions, to evaluate the performance of the proposed approach. To their surprise, manually created collections did not perform well and significantly decreased the accuracy of the generated collections.

Overview: Walter, F. E. et al., 2012 provided a technical and business perspective of recommender systems by combining ubiquitous systems with a market study. Customers were provided with personalized products via a store owned mobile device known as Personal shopping assistant (PSA). They investigated the extent of benefits gained by retail stores with the use of a tailored recommender system named as LARS (Layered architecture for recommender systems).

Strength: The model assisted the customers by suggesting alternatives or complementary products of the demanded merchandise. Their study proved that the recommender system can outperform even if it considers the real-time context of purchase rather than pre-stored and processed individual customer's profile. Therefore, the cutting-edge solution, A model that was flexible to changes, provided by Walter, F. E. et al., 2012 can be considered as one step forward in the field of recommendations. 
Weakness: Walter, F. E. et al., 2012 delivered the precise collection of data and personalized results in retail stores. The only challenge in their solution was customer loyalty fluctuations due to changing user preference. Moreover, this approach could only be effective if the retailer has no information on consumer behavior patterns.

Overview: Hanke, J. et al., 2018 researched to introduce an adaptive recommendation system to leverage the functionalities of fitting rooms for fashion retailers. Their framework suggested recommendations to the customers based on additional features such as weather conditions, location trends.

Strength: The authors in Hanke, J. et al., 2018 proposed a novel recommender system of smart fitting rooms that offered suggestions of garments to the user on the screens inside individual trial room cabins of fashion stores. They undertook the problem in steps i.e. first by detecting the customer and products present in smart fitting rooms and then increasing the product recommendation quality based on contextual information of the product along with customer (e.g., store type, weather conditions) via a transaction dataset from a leading German fashion retailer.

Weakness: In their study, they targeted the contextual information of the sales, which require a more comprehensive evaluation that can lead to improved recommendations. Additional sensors that collect data such as surrounding activities, could yield much better suggestions of products that customers will end up buying.

Overview: Keller, T., \& Raffelsieper, M., 2014 focused their study on bricks and mortar stores. Online stores can trace, and track user's information based on clicks on the web page. However, in-stores sales only have data at the point the customer makes the final purchase. They introduced two tools, one in the form of a mobile application for the 
customers to share recommendations with others and the second tool for retailers with which they can acquire knowledge about their customers beyond the scope of Receipt Horizon.

Strength: They implemented their three recommendation systems that exploit different areas. The first system was based on the customer's fluctuating demand and interest in popular items. The second recommendation system was based on the previous purchase history of users. The last implemented recommendation had a novel feature that allowed customers to share the product pictures and specifications with friends and family over the application.

Weakness: As the author tried to bring online store tracking advantages to the proposed solution, however, the model still lacked in comparison to the advantages of click data collected online. The author mentioned that customers can be tracked online even if they do not buy anything from the website, yet the click data analysis remained undiscussed.

Overview: While addressing the problem of big data being generated in the e-commerce business, Sarwar, B. M. et al., 2002 evaluated the clustering technique to enhance the scalability of recommender systems. They suggested a clustering-based neighborhood approach deliver equivalent prediction quality as of collaborative fitting by considering neighborhoods.

Strength: While addressing the performance aspect, the authors in Sarwar, B. M. et al., 2002 claimed that their algorithm tends to deliver uniform-sized clusters computed using the Pearson-r correlation coefficient between users. Their solution model greatly enhanced the online recommendations that helped consumers to find specific items. 
Weakness: But while computation they neglected the fact that splitting the data population into clusters might compromise the accuracy of the recommendation to the users which are near the peripheries of the cluster they have been assigned to. To eliminate the accuracy reduction factor, Pre-clustering could be embraced.

Overview: Wong, W. K. et al., 2012 introduced a novel approach based on a radio frequency identification system that integrated the product specifications with customer's previous purchase for a personalized recommendation to the users and effective crossselling which enhanced sales performance of the retailers.

Strength: Wong, W. K. et al., 2012 projected an innovative idea by developing two different systems built on rule-based fuzzy logic. The first one, Smart Dressing system aided by radio frequency identification technologies to provide information like product identification, customer preference and offers cross-product suggestions. On the other hand, an Intelligent product cross-selling system termed as a hybrid intelligent system was developed to integrate customer preference with other products for the mix and match clothes shopping.

Weakness: Their model provided recommendations based on the items that are currently available in stores, but in real-life situations decision-making process in terms of matching clothes and apparels is manifold. The fuzzy screening technique used in this model handled imprecise and vague decisions and thus requires further evaluation.

\subsubsection{Association Rule Mining based on historical data}

As defined by Agarwal, R., \& Srikant, R., 1994, the Apriori algorithm is a technique to analyze the items in the basket and generate knowledge base rules for associating with other products available such as if you are buying diapers, then you may also like to buy 
beer (Kelly, F., 2002). In the year 2018, Sagin, A. N., \& Ayvaz, B., 2018 determined association rules to understand the cross-purchasing behavior of customers. They split the dataset into two parts and exploited the Apriori algorithm as well as FP growth using the data mining tool WEKA. They compared the result outcomes of both algorithms and stated that the difference was generated by both approaches. The purchase trend and rules derived from both algorithms showed resemblance.

Overview: Han, J. et al., 2000 proposed an innovative data structure of frequent pattern tree (FP-tree) to store the significant patterns of the data by inventing a new pattern growth method named FP-growth. They compared FP-growth with other approaches like Apriori in terms of cost and storage efficiency and mining flexibility.

Strength: The main contribution of their study is three-fold, which proved the scalability and efficiency of their novel model structure. First, they condensed the large dataset to reduce the cost associated with database scans. Next, to avoid generating cost consuming candidate sets, they embraced the pattern fragment growth method. Lastly, to minimize the space utilized in search, they employed divide and conquer technique to segregate the mining tasks into smaller chunks.

Weakness: However, in most instances, FP-tree fritters memory and incapacitate to fit. Therefore, it demands large input-output disks to read and write FP-trees. Hence, mining frequent datasets through these intermediate databases is a time-consuming factor. Moreover, the major issue with frequent data mining patterns is that it considers each item as of the same weight/value. But, in real life supermarkets, all items have different profit margins, such as margin overselling a shampoo bottle is less than that of a Microwave. 
Therefore, traditional frequent pattern mining in a database is unable to find the set of valuable users/itemsets that plays a major role in increasing profits of the business.

Overview: By incorporating association rule mining with a collaborative recommender system, Lin, W. et al., 2002 designed a new algorithm that adjusts generated rules according to the target range, instead of minimum support. This algorithm generates less but powerful recommendation rules which reduce computation time as well.

Strength: The recommendation system presented by Lin, W. et al., 2002 uses enough association rules for each consumer with respect to minimum support. Their proposed algorithm generates rules associated with each item and each user. The system uses item associations if the minimum support value is less than the threshold, otherwise, it uses user association rules.

Weakness: However, to find the precise association rules that match the user's profile to the best, a sliding window is used in order to extract personalized rules based on target users. Each time the size of the window is decreased to skim out the three best-fit rules. This procedure to generate association rules require an iterative search of rule base and hence increases the CPU head.

Overview: To comprehend the problem of mining large databases of sales transactions, Savasere, A. et al., 1995 presented a new algorithm "Partition" which scanned databases just twice before generating effective association rules. They compared the efficiency of their proposed solution, which outperformed the Apriori algorithm.

Strength: For the data memory compatibility, their proposed algorithm divided the database into small portions. In the first scan of the dataset, the algorithm generated all the 
item sets that were appearing frequently in partitioned data, while the second data scan generated global support for the frequent itemset obtained before.

Weakness: The structure of their proposed solution reduced the computation load on the CPU and increased speediness. However, while computing long patterns, the Partition approach may perform less efficiently as it always generates more item sets than the Apriori algorithm.

Overview: Chen, M. C. et al., 2005 showed their interest in finding the behavior of the customers. Significantly, their approach to making decisions is based on analyzing situations and patterns. They extracted customer's behavior values such as demographical value, from the databases of retail marketing using data mining technologies to detect changes in customer behavior.

Strength: To rapidly generate market strategies based on customer behavior, they developed an online query system that provides informational feedbacks based on customer variables. Authors initially integrated customer values with the growth matrix and then used association rules to generate patterns between customer profiles and products using the Recency-Frequency-Monetary Value approach (Miglautsch, J. R., 2000).

Weakness: The dynamic behavior of databases demands the stability of the model in case behavior pattern changes over time, otherwise it might lead to less efficient association rule generation. The study proposed by the author interpreted the behavior by exploiting historical transactional databases. However, their problem solution required a self decomposable technique that monitors the changing patterns and extracts new rules after decomposing the old pattern rules to maintain performance consistency. 
Overview: Recently Safitri, A., 2019 analyzed digital printing business using the Apriori algorithm implemented on Tanagra 1.4 (Rakotomalala, R., 2005) to understand customer behavior. The objective of the study was to predict the favorite item patterns of the clients so that they can target a certain group of people with the most popular item that will directly increase their production as well as the profit of the business.

Strength: The motive of their study was to eliminate the manual processes utilized in establishing business approaches. The model used a basic Apriori algorithm that generated the products which were high in demand. Their model results were used to comprehend customer's behavior with the aim to provide personalized services.

Weakness: However, due to smaller datasets, their experiment inferred that two items are highly correlated. Such a specific correlation in itemset will lead to a congested market. Thus, the experiment results could differ while evaluating higher business production capacity.

\subsubsection{Machine learning techniques}

The growth of electronic commerce (e-commerce) has substantially increased in recent years, which has led companies to seek new ways to reach new users and retain their existing customers. Each website is competing against each other to provide more personalized and cost-efficient deals to its customers to eliminate the factor of churning. Overview: Considering this budding issue, Kumar, A. et al., 2019 investigated customer behavior to understand the intentions of repurchase done online. Their study is inferred theoretical solution by analyzing the shopping malls context with respect to customer's behavioral patterns. Further, the research observation integrated the theoretical solution to online platforms in order to predict the repurchase intentions of online consumers. 
Strength: To execute their experiment work, authors exploited five machine learning classification techniques i.e. Decision trees (C5.0), AdaBoost, random forest (RF), support vector machine (SVM), and neural network (NN) in different settings. According to the findings of their experiment, the AdaBoost classification model generated higher accuracy and sensitivity of $97.58 \%$ and $0.95 \%$ respectively.

Weakness: The focus of their study was to interrogate customer behavior in association with online purchases. The author neglected many other factors that could have been used to increase the quality of the work by prioritizing these attributes in an efficient manner such as e-service provider's details and ranking interactive features of websites (Kim, $\mathrm{H}$. W., \& Gupta, S., 2009).

Overview: XingFen, W. et al., 2018, proposed scenario-based optimization algorithms that integrated logistic regression with the XGboost algorithm with the intention of predicting user's purchasing behavior on an e-commerce website. The objective of their research was to analyze the purchase pattern of specific products in a certain period. The result of their study could provide significant enhancement in the marketing of e-commerce platforms and to discover potential buyers.

Strength: Their experimental setup used three predictive models i.e. logistic regression, XGBoost, and finally XGBoost integrated with the logistic regression model. In an integrated setup, XGBoost was used for feature transformation which produced new feature vectors. Each new vector feature was considered as an input factor for the logistic regression model. The result of the integrated model generated more scalable and feasible results than models used individually. 
Weakness: Authors managed to generate superior and feasible results using an integrated model, but the problem of slow parameter search in the XGBoost algorithm is unattended. Artificial Chemical Reaction Optimization Algorithms (ACROA) could be implemented to optimize the parameters of the XGBoost model as suggested in Jiang, Y. et al., 2019.

Overview: Sakar, C. O. et al., 2019 also investigated customer browsing behavior within a specific E-commerce website intending to analyze the purchase conversion rate. They proposed a system that analyses real-time online shopper behavior. Their study was divided into two separate modules. The first module analyzed pageview data that contained sessions and user information. By gathering the knowledge of pageview data, they predicted the purchase intentions of the users using data mining techniques.

Strength: The second module of the study used a time series analysis of sequential clickstream data. This module was intended to generate a probability estimate of visitors who would leave the website without placing an order by training long short-term memory based recurrent neural networks. In conclusion, the Authors integrated the results of these two modules to determine a user's purchase conversion rates.

Weakness: The knowledge assembled in the first module of their study was used to generate offers by analyzing the user's intention of purchase. Considering the technology enhancement of this era, their novel solution could have improved the conversion rates by providing personalized suggestions to the users on various platforms as discussed in Grbovic, M. et al., 2015.

Overview: Tahiri, N. et al., 2019 developed a machine learning model to forecast the products that will be purchased by the users in the future along with the store location associated with the next shopping trip. The reason for their novelty is that they considered 
the purchase history of the user accompanied by promotional schemes of the distinct stores and their distance from the user's residence in the procedure of prediction.

Strength: To design the desired prediction model, the Authors exploited deep neural network algorithms. They applied recurrent neural networks along with forward propagating neural networks at the learning stage of their model. Their experiment of predicting the current consumer's basket was carried out using the three data mining models: Long short-term memory model (LSTM), Non-negative matrix factorization network (NNMF), and Gradient Boosted Tree (GBT).

Weakness: Due to the limitation in the size of the dataset, they simulated the real data and augmented the real dataset. The amplification of data led to an increase in the accuracy of the model from $27 \%$ to $49 \%$. However, the quality of the model could have been increased if the model allowed an influx of new products or user entries in the database.

Overview: Gupta, V. et al., 2014 presented an interesting work that aims towards understanding consumer purchase intent based on their social media interactions. The authors evaluated their proposed model solution on a linear SVM model using the area under the curve metric measurement. While training their two data sets, they achieved a 0.93 and 0.89 AUC score.

Strength: To approach the problem solution, they target minute details of the social posts uploaded by the users. They gained information from text, incorrect grammar, language ambiguity, and presented a method to extract n-gram features using WordNet similarity to train linear SVM model. To provide resilience to their proposed model, they evaluated two entirely different datasets obtained from distinct websites. 
Weakness: Gupta, V. et al., 2014 presented a novel approach to assess consumer behavior, however, they binary categorized to purchase intent. From their solution perspective, the model captured the intent availability in the text, but the type of intent was left unclear. By figuring the intent class they could provide better foresight of their model's potential.

Overview: Haque, R. et al., 2019 worked on the related problem of measuring the purchase probability of the product or services by analyzing Twitter data. They proposed an approach based on recurrent neural networks, but the tangible novelty of their work was reflected by the semi-automatic technique to create a training dataset.

Strength: For their model evaluation, they addressed the class imbalance problem and measured the performance based on F-score to generate meaningful information. They compared the results of their proposed LSTM model with other base classifiers such as decision trees, logistic regression, random forest, and Naïve Bayes to provide a substantial base to their high achieved F1-score (83\%).

Weakness: The prediction focus of Haque, R. et al., 2019 was to classify purchase intents of the twitter user. However, their model evaluated a high precision rate and sub-standard recall rate. Therefore, their model correctly identified purchase intent tweets but struggled in identifying non-purchase intent tweets.

Overview: Ding, X. et al., 2015 presented a domain adaption model with the aim of mining intentions of social media users to purchase domain related products. They evaluated the model on a dataset collected from the Chinese microblogging website- Sina Weibo, with the purpose to provide better recommendations to the consumers by targeting advertisements. 
Strength: The author exploited the convolutional neural network architecture to propose the novel consumption intention mining model. Their model identifies the purchase intention of one product and retains the knowledge in the last layer of the model to identify the intention of consumption for the next product category. They compared their model performance with the baseline model and achieved a significant increase in accuracy metric.

Weakness: While model evaluation, they considered accuracy as their main performance assessment metric. However, they neglected the statistics of class imbalance. They mentioned that only 625 out of 1000 posts show the direct intention of purchase which results in a high imbalance in their data classification. Hence, measuring the accuracy of the experiments might have obscured the meaningful information

Overview: A novel technique based on lexicon-semantic patterns was introduced by Hamroun, M. et al., 2015 for leveraging the commercial business. They studied the consumption intention of the users via microblog posts. Precision and Recall were used as evaluation metrics, and they achieved a maximum of $55.59 \%$ and $55.28 \%$, respectively.

Strength: The approach mentioned in Hamroun, M. et al., 2015 did not rely on manual word syntactic, rather they proposed ontology relationships in the words to understand the intentions of the customers through online reviews provided. The patterns extracted were then used as features for the classification process. They applied their approach to five different datasets to validate the superiority of the model.

Weakness: The author did not provide any imbalanced data aspect related to the tweets. Their model worked consistently on pre-defined stable data which depicts the lack of data- 
processing and cleaning of other datasets used. Moreover, the method proposed lacks in extracting the domain-specific knowledge out of the text, for example, if the tweet is classified in the intent category, the label is intent remains unclear.

Overview: Recently, Atouati, S. et al., 2020 presented a wonderful framework that stands out of other related works done in the field of assessing consumer intentions using social media data. They worked on twitter posts related to British Airways to comprehend the intentions of the flight users.

Strength: The authors presented a novel technique to classify the posts that indicate negative purchase intent by employing the word dependency parsing tool to collect negations in the text. They exploited the logistic regression algorithm and provided a customized cost function for the algorithm that is inherited from the neural networks.

Weakness: In Atouati, S. et al., 2020, they introduced a superiorly structured solution model that recognized each aspect related to knowledge extraction from social media data. They worked on identifying specific intent class (negative purchase intention), which no doubt is an addition to the previous related work. However, they could have extended their branch in specifying positive intent class as well by following the same structure model. Thus, their work provides a significant research baseline for the model extension.

\subsubsection{Other major analysis done on Grocery basket recommendation}

The correlative association among items is a focus of interest in some prior works. The study of correlation includes association among items within a basket ( $L i$, M. et al., 2009) as well as across baskets (Le, D. T. et al., 2017). Overview: To recommend an item that could be added to the basket after investigating the relation of items already in the 
basket, Le, D. T. et al., 2017 exploited two different approaches based on matrix factorization and association rules. The former approach Basket-Sensitive factorization machine (BFM) worked on user information associated with the item in basket to target the next item recommendation. Whereas the later Constrained Basket-Sensitive factorization machine (CBFM) that further incorporated constraints on the basket with similar content.

Strength: The framework proposed by the authors in Le, D. T. et al., 2017 addressed the online shopping behavior of the users. The motive of their research was to generate personalized product relationship rules that in return reduced product exploration time spent by the customer. To validate the superior performance achieved by their solution, they exploited three real-time datasets reflecting product-to-product associations.

Weakness: CBFM can infiltrate deep into the relationship between the items that are currently in the basket of the user. However, their approach neglected the unbalanced ratio of hot items to cold items that are not considered while providing recommendations using the rule-based method. These models fail to capture the detailed semantics of the items because these algorithms are optimized for global recommendation metrics.

Overview: To increase the number of products in a basket, Trofimov, I., 2018 proposed an unsupervised statistical model that provides recommendations of complementary products to the users based on browsing history information. The author exploited the cold start problem by introducing the BB2vec model (an extension of the prod2Vec model by Vasile, F. et al., 2016) which analyzed data from Baskets and Browsing sessions.

Strength: The proposed model framework in Trofimov, I., 2018 used a novel technique for product recommendations. The model trained itself using vector representations assigned 
to each product by scrutinizing user baskets and browsing the product. The main goal of the research was to deliver improved and personalized recommendations to consumers.

Weakness: This approach joined the basket item data with browsing history to rank the process of learning for training the model. The only shortcoming of this study was, it provided a recommendation for products which had few or no purchase history. While suggesting products with high purchase history, BB2vec is no different than other existing models.

Overview: Wan, M., et al., 2018 proposed an embedded based recommendation for grocery shopping by introducing a triple2vec approach which was based on transactional data of the same basket linked by item, item, user compatibility. Their main idea was to leverage the loyalty and complementarity of the items represented. They also proposed an algorithm named adaLoyal which provided personalized grocery product recommendations to the users determined by a mixture of frequency-based behaviors.

Strength: To begin with, they exploited rich datasets which included transactional details of various types of grocery stores, online shopping websites, supermarkets. The authors also presented the quantifiable proof of the model supremacy in terms of recommendation. Their qualitative analysis claimed that to comprehend customer loyalty, item-to-item interactions deliver more useful results than cross-basket relationships.

Weakness: Their approach was applicable in the scenarios where short-term item dependencies of the items were to be considered. But the study direction may not focus on the process which considers the evaluation of preference history. Hence, other approaches like Recurrent Neural networks (RNN) were introduced to this field to capture sequential long-term dependencies (Yu, F. et al., 2016). 
Overview: $Y u$, F. et al., 2016 to target the next basket prediction, proposed an innovative model based on recurrent neural networks. The RNN model was provided with the values inferred from the set of items to which users interact in sequence and hence, accordingly the next recommended items are ranked.

Strength: The authors proposed the Dynamic Recurrent bAsket Model (DREAM) that captured the dynamic transactional information of the user and sequential patterns of purchase history. They aimed to embed formerly purchased items for the current product interest forecast. They applied their model to two different datasets and claimed the model to be a performance enhancer for basket recommendation.

Weakness: The Model proposed by $Y u$, F. et al., 2016 was based on the RNN approach that exploited recurrent models to investigate the active preferences of the users by capturing checkout events. However, this approach overlooked the static preferences of customers. Transactional data of previous months are not equally weighted as recent purchase data. A more sophisticated RNN model such as Long-Term Memory Model could be exploited to capture feedbacks from earlier sequences to generate better recommendations.

Overview: Buser, D. C., 2007 also concentrated on recommendations in grocery stores. The main model was based on the RFID (radio frequency identification) infrastructure. To enhance the economic feasibility for both, the retailer and the customer, the proposed architecture of the study contained personal shopping assistance and a back-end system acting as retailer's information databases.

Strength: The authors in Buser, D. C., 2007 tried to apply online recommender systems to conventional stores. They proposed an application architecture that demonstrated its 
usefulness for customers as well as retailers. After comparing the benefits of both entities, the authors proposed a conceptual framework of a device that allowed companies to evaluate customer's information and recommender systems.

Weakness: Their framework, a personal assistant integrated with a shopping cart, detected the products with RFID tags, and suggested personalized products to the users. This architecture required producers to integrate each product with RFID (radio frequency identification) tags. Incorporating such technical revolution in-stores could affect customers as well as producer loyalty due to digital division.

Overview: Raorane, A. A. et al., 2012 exploited market basket analysis to understand the behavior of the customer. This research introduced clustering and k-means as a data mining model to explore shopping data. The investigators did experimental analysis by commissioning association rules using market basket analysis, which improved the approach of arranging products on the shelf to fetch more profit to the seller.

Strength: The author exploited the baseline Apriori algorithm to evaluate the transactional history of the consumers. They exploited a large dataset consisted of the purchase information of the customers from a supermarket in India, which was consisted of vast label descriptions. Support and Confidence were considered as the metrics of measurement. Weakness: The target of increasing sales was achieved but the dynamic state of consumer behavior was a point of concern in their research. To withstand the competitive market, organizations must concentrate on change in consumer behaviors with respect to time. Moreover, their approach is incapable to exploit the shopping trends and customer's behavior patterns. 


\subsection{Comparative Analysis}

This segment describes the comparative analysis that is compressed in Table 6. This comparison table consists of five columns for each row. The first column is to declare the name of the author and year of publication of the work. The second column explains the dataset used for the research done by the author. The third column concludes the task or purpose of the research work. The proposed solution to the problem is explained in the fourth column of the table. This column provides the novel result proposed for the solution design. The last column describes the technology used as a baseline by the researchers.

Table 6. Comparative Analysis table

\begin{tabular}{|c|c|c|c|c|}
\hline Related Work & Dataset & Task & Proposed Solution & Technology Used \\
\hline $\begin{array}{l}\text { Wölbitsch, } M . \\
\text { et al., } 2019\end{array}$ & $\begin{array}{l}\text { Anonymous } \\
\text { dataset of } 2 \text { years } \\
\text { collected from } 20 \\
\text { stores of } 4 \text { fictional } \\
\text { cities }\end{array}$ & $\begin{array}{l}\text { Product } \\
\text { recommendations for } \\
\text { traditional brick-and- } \\
\text { mortar retailers }\end{array}$ & $\begin{array}{l}\text { Recommendation } \\
\text { system for brick-and- } \\
\text { mortar retailers }\end{array}$ & $\begin{array}{l}\text { Radiofrequency } \\
\text { Identification, Data } \\
\text { augmentation }\end{array}$ \\
\hline $\begin{array}{l}\text { Barkan, O., \& } \\
\text { Koenigstein, } \\
\text { N., } 2016\end{array}$ & $\begin{array}{l}\text { Microsoft Xbox } \\
\text { Music service data, } \\
\text { Microsoft Store } \\
\text { sales data }\end{array}$ & $\begin{array}{l}\text { To compare } \\
\text { Item } 2 \text { Vec and SVD- } \\
\text { based item similarity } \\
\text { model }\end{array}$ & $\begin{array}{l}\text { Neural embedded } \\
\text { algorithm: Item } 2 \text { vec }\end{array}$ & $\begin{array}{l}\text { Collaborative filtering } \\
\text { under Skip-gram with } \\
\text { Negative Sampling } \\
\text { approach (SGNS) } \\
\text { (Mikolov, } \text { T. et al., } \\
\text { 2013) }\end{array}$ \\
\hline $\begin{array}{l}\text { Grbovic, M. et } \\
\text { al., } 2015\end{array}$ & $\begin{array}{l}\text { Dataset of e-mail } \\
\text { receipts from } \\
\text { Yahoo }\end{array}$ & $\begin{array}{l}\text { Product } \\
\text { recommendations in } \\
\text { the form of ads }\end{array}$ & Prod2vec approach & $\begin{array}{l}\text { Natural language } \\
\text { processing }\end{array}$ \\
\hline $\begin{array}{l}\text { Vasile, F.et } \\
\text { al., } 2016\end{array}$ & Music open dataset & Next song prediction & $\begin{array}{l}\text { Meta-prod2vec } \\
\text { algorithm }\end{array}$ & $\begin{array}{l}\text { Matrix factorization } \\
\text { methods }\end{array}$ \\
\hline $\begin{array}{l}\text { Grbovic, M., \& } \\
\text { Cheng, H., } \\
2018\end{array}$ & $\begin{array}{l}\text { Dataset of rental } \\
\text { market Airbnb }\end{array}$ & $\begin{array}{l}\text { Recommendation for } \\
\text { the short-term rental } \\
\text { marketplace }\end{array}$ & $\begin{array}{l}\text { Listing and user } \\
\text { Embedding }\end{array}$ & $\begin{array}{l}\text { Skip-gram modeling } \\
\text { technique }\end{array}$ \\
\hline $\begin{array}{l}\text { Chen, T. et al., } \\
2017\end{array}$ & $\begin{array}{l}\text { User-bookmarking- } \\
\text { article behavior } \\
\text { data from } \\
\text { CiteULike.org, } \\
\text { Yahoo! News Feed } \\
\end{array}$ & $\begin{array}{l}\text { Content-based } \\
\text { recommendation for } \\
\text { completely new texts. }\end{array}$ & $\begin{array}{l}\text { Unified (Supervised } \\
\text { + Unsupervised) joint } \\
\text { text embedding } \\
\text { model }\end{array}$ & $\begin{array}{l}\text { Supervised text } \\
\text { embedding }\end{array}$ \\
\hline $\begin{array}{l}\text { Mohan, A. et } \\
\text { al., } 2019\end{array}$ & $\begin{array}{l}\text { Data of alpha-test } \\
\text { users of the } \\
\text { application } \\
\text { "SmartEmma" }\end{array}$ & $\begin{array}{l}\text { Recommendation } \\
\text { system in the } \\
\text { Grocery shop } \\
\text { application }\end{array}$ & $\begin{array}{l}\text { Evaluation of a } \\
\text { business intelligence } \\
\text { platform. }\end{array}$ & $\begin{array}{l}\text { Item-item collaborative } \\
\text { filtering }\end{array}$ \\
\hline
\end{tabular}




\begin{tabular}{|c|c|c|c|c|}
\hline $\begin{array}{l}\text { Kouki, P. et } \\
\text { al., } 2019\end{array}$ & $\begin{array}{l}\text { Data from the } \\
\text { Home Depot online } \\
\text { retailer }\end{array}$ & $\begin{array}{l}\text { Collections of similar } \\
\text { theme products }\end{array}$ & $\begin{array}{l}\text { Leveraging domain } \\
\text { knowledge and } \\
\text { transactional data }\end{array}$ & Text embedding \\
\hline $\begin{array}{l}\text { Walter, F. E. et } \\
\text { al., } 2012\end{array}$ & Anonymous & $\begin{array}{l}\text { Efficient } \\
\text { recommender } \\
\text { systems for retail } \\
\text { stores }\end{array}$ & $\begin{array}{l}\text { Personal shopping } \\
\text { assistant for } \\
\text { personalized } \\
\text { suggestions }\end{array}$ & $\begin{array}{l}\text { Layered architecture } \\
\text { for recommender } \\
\text { systems }\end{array}$ \\
\hline $\begin{array}{l}\text { Hanke, J. et } \\
\text { al., } 2018\end{array}$ & $\begin{array}{l}16 \text { months data of a } \\
\text { German fashion } \\
\text { retailer }\end{array}$ & $\begin{array}{l}\text { Product } \\
\text { recommendation } \\
\text { systems for fashion } \\
\text { stores }\end{array}$ & $\begin{array}{l}\text { Recommendation } \\
\text { algorithms tailored to } \\
\text { the sensing } \\
\text { capabilities of smart } \\
\text { fitting rooms }\end{array}$ & $\begin{array}{l}\text { Bayesian Probabilistic } \\
\text { Ranking, Association } \\
\text { rule Mining }\end{array}$ \\
\hline $\begin{array}{l}\text { Keller, T., \& } \\
\text { Raffelsieper, } \\
\text { M., } 2014\end{array}$ & $\begin{array}{l}\text { clickstream data } \\
\text { collected from } \\
\text { webpages alongside } \\
\text { the actual purchase } \\
\text { data }\end{array}$ & $\begin{array}{l}\text { Comparable data } \\
\text { collection platform } \\
\text { and a comparable } \\
\text { customer interaction } \\
\text { platform. }\end{array}$ & $\begin{array}{l}\text { Term Receipt } \\
\text { Horizon, Leveraged } \\
\text { mobile app }\end{array}$ & $\begin{array}{l}\text { Amazon's Movers \& } \\
\text { Shakers algorithm }\end{array}$ \\
\hline $\begin{array}{l}\text { Sarwar, B. M. } \\
\text { et al., } 2002\end{array}$ & $\begin{array}{l}\text { Data from } \\
\text { MovieLens }\end{array}$ & $\begin{array}{l}\text { To improve the } \\
\text { scalability of } \\
\text { recommender } \\
\text { systems }\end{array}$ & $\begin{array}{l}\text { Clustering-based } \\
\text { neighborhood } \\
\text { approach }\end{array}$ & $\begin{array}{l}\text { Pearson nearest } \\
\text { neighbor algorithm, } \\
\text { Collaborative filtering }\end{array}$ \\
\hline $\begin{array}{l}\text { Wong, W. K. et } \\
\text { al., } 2012\end{array}$ & $\begin{array}{l}\text { Deployed in fashion } \\
\text { retailing company } \\
\text { of Hong Kong }\end{array}$ & $\begin{array}{l}\text { Smart Dressing } \\
\text { System, Intelligent } \\
\text { product cross-selling } \\
\text { system }\end{array}$ & $\begin{array}{l}\text { Intelligent system } \\
\text { integrated with the } \\
\text { RFID technology }\end{array}$ & $\begin{array}{l}\text { Fuzzy screening } \\
\text { technique }\end{array}$ \\
\hline $\begin{array}{l}\text { Sagin, A. N., \& } \\
\text { Ayvaz, B., } \\
2018\end{array}$ & $\begin{array}{l}\text { Dataset of the } \\
\text { domestic hardware } \\
\text { market }\end{array}$ & $\begin{array}{l}\text { To identify product } \\
\text { groups that tended to } \\
\text { be sold together }\end{array}$ & $\begin{array}{l}\text { Apriori algorithm as } \\
\text { well as FP growth }\end{array}$ & $\begin{array}{l}\text { Market basket analysis } \\
\text { technique }\end{array}$ \\
\hline $\begin{array}{l}\text { Han, J. et al., } \\
2000\end{array}$ & Not applicable & $\begin{array}{l}\text { To develop a pattern } \\
\text { growth method for a } \\
\text { transaction database }\end{array}$ & $\begin{array}{l}\text { Frequent pattern } \\
\text { growth }\end{array}$ & $\begin{array}{l}\text { Frequent pattern tree } \\
\text { (FP-tree) structure }\end{array}$ \\
\hline $\begin{array}{l}\text { Lin, W. et al., } \\
2002\end{array}$ & EachMovie Dataset & $\begin{array}{l}\text { To mine association } \\
\text { rules of classification } \\
\text { type }\end{array}$ & $\begin{array}{l}\text { New collaborative } \\
\text { recommendation } \\
\text { technique }\end{array}$ & $\begin{array}{l}\text { Association rule } \\
\text { mining algorithms }\end{array}$ \\
\hline $\begin{array}{l}\text { Savasere, A. et } \\
\text { al., } 1995\end{array}$ & $\begin{array}{l}\text { Six synthetic } \\
\text { datasets }\end{array}$ & $\begin{array}{l}\text { An efficient method } \\
\text { to discover } \\
\text { association rules in } \\
\text { large datasets }\end{array}$ & Partition algorithm & $\begin{array}{l}\text { Association rule } \\
\text { mining algorithms }\end{array}$ \\
\hline $\begin{array}{l}\text { Chen, M. C.et } \\
\text { al., } 2005\end{array}$ & $\begin{array}{l}\text { Sample database of } \\
\text { Foodmart }\end{array}$ & $\begin{array}{l}\text { To integrate } \\
\text { customer behavioral } \\
\text { variables in mining } \\
\text { association rules }\end{array}$ & Online query system & $\begin{array}{l}\text { Recency-Frequency- } \\
\text { Monetary Value } \\
\text { approach }\end{array}$ \\
\hline $\begin{array}{l}\text { Safitri, } A \text {., } \\
2019\end{array}$ & $\begin{array}{l}\text { Data from the } \\
\text { digital printing } \\
\text { business }\end{array}$ & $\begin{array}{l}\text { Customer behavior } \\
\text { analysis }\end{array}$ & $\begin{array}{l}\text { The Open Group } \\
\text { Architecture } \\
\text { Framework } \\
\text { (TOGAF) }\end{array}$ & Apriori Algorithm \\
\hline $\begin{array}{l}\text { Le, D. T. et al., } \\
2017\end{array}$ & $\begin{array}{l}\text { Three public real- } \\
\text { life datasets: } \\
\text { TaFeng, BeiRen, } \\
\text { Foursquare }\end{array}$ & $\begin{array}{l}\text { To provide } \\
\text { personalized } \\
\text { recommendations for }\end{array}$ & $\begin{array}{c}\text { Basket-Sensitive } \\
\text { factorization } \\
\text { machine, Constrained }\end{array}$ & $\begin{array}{l}\text { Matrix factorization } \\
\text { and association rules }\end{array}$ \\
\hline
\end{tabular}




\begin{tabular}{|c|c|c|c|c|}
\hline & & $\begin{array}{l}\text { a user's current } \\
\text { basket }\end{array}$ & $\begin{array}{l}\text { Basket-Sensitive } \\
\text { factorization machine }\end{array}$ & \\
\hline $\begin{array}{l}\text { Trofimov, I., } \\
2018 .\end{array}$ & $\begin{array}{l}4 \text { datasets with user } \\
\text { behavior log on e- } \\
\text { commerce web sites }\end{array}$ & $\begin{array}{l}\text { Products } \\
\text { recommendation } \\
\text { using Baskets and } \\
\text { Browsing sessions }\end{array}$ & BB2vec model & prod2Vec model \\
\hline $\begin{array}{l}\text { Wan, M. et al., } \\
2018\end{array}$ & $\begin{array}{l}\text { Two public and two } \\
\text { proprietary grocery } \\
\text { datasets }\end{array}$ & $\begin{array}{l}\text { To Recommend } \\
\text { products for grocery } \\
\text { shopping }\end{array}$ & $\begin{array}{l}\text { triple2vec and } \\
\text { adaLoyal }\end{array}$ & $\begin{array}{l}\text { Skip-gram modeling } \\
\text { technique }\end{array}$ \\
\hline $\begin{array}{l}\text { Yu,F. et al., } \\
2016\end{array}$ & $\begin{array}{l}\text { Two real-world } \\
\text { datasets, i.e., Ta- } \\
\text { Feng1 and T-mall }\end{array}$ & $\begin{array}{l}\text { Next basket } \\
\text { recommendation }\end{array}$ & $\begin{array}{l}\text { Dynamic REcurrent } \\
\text { bAsket Model } \\
\text { (DREAM) }\end{array}$ & $\begin{array}{l}\text { Recurrent Neural } \\
\text { Network (RNN) }\end{array}$ \\
\hline $\begin{array}{l}\text { Buser, D. C., } \\
2007\end{array}$ & Not Applicable & $\begin{array}{l}\text { Recommendations in } \\
\text { grocery stores }\end{array}$ & $\begin{array}{l}\text { Personal shopping } \\
\text { assistance and a back- } \\
\text { end system }\end{array}$ & $\begin{array}{l}\text { RFID (radio frequency } \\
\text { identification) } \\
\text { infrastructure }\end{array}$ \\
\hline $\begin{array}{l}\text { Raorane, } A . A . \\
\text { et al., } 2012\end{array}$ & $\begin{array}{l}\text { Transactional data } \\
\text { of a supermarket in } \\
\text { Maharashtra India }\end{array}$ & $\begin{array}{l}\text { For arranging } \\
\text { products on the shelf } \\
\text { in order to fetch more } \\
\text { profit }\end{array}$ & $\begin{array}{l}\text { Clustering and k- } \\
\text { means as a data } \\
\text { mining model }\end{array}$ & $\begin{array}{l}\text { Market basket analysis } \\
\text { algorithm }\end{array}$ \\
\hline $\begin{array}{l}\text { Kumar, A. et } \\
\text { al., } 2019\end{array}$ & $\begin{array}{l}\text { Data in the form of } \\
\text { a structured } \\
\text { questionnaire held } \\
\text { online/offline in } \\
\text { metro cities of India }\end{array}$ & $\begin{array}{l}\text { To predict the } \\
\text { repurchase intentions } \\
\text { of an online } \\
\text { consumer }\end{array}$ & $\begin{array}{l}\text { Decision trees, } \\
\text { AdaBoost, Random } \\
\text { forest (RF), Support } \\
\text { vector machine } \\
\text { (SVM) and Neural } \\
\text { network (NN) }\end{array}$ & $\begin{array}{l}\text { Data mining } \\
\text { techniques, Artificial } \\
\text { bee colony (ABC) } \\
\text { algorithm }\end{array}$ \\
\hline $\begin{array}{l}\text { XingFen, W. et } \\
\text { al., } 2018\end{array}$ & $\begin{array}{l}3 \text { Months dataset of } \\
\text { an anonymous e- } \\
\text { commerce website }\end{array}$ & $\begin{array}{l}\text { To predict user's } \\
\text { purchasing behavior }\end{array}$ & $\begin{array}{l}\text { Optimized XGBoost- } \\
\text { LMT }\end{array}$ & $\begin{array}{l}\text { Logistic regression and } \\
\text { XGBoost algorithm }\end{array}$ \\
\hline $\begin{array}{l}\text { Sakar, C. O. et } \\
\text { al., } 2019\end{array}$ & $\begin{array}{l}\text { Clickstream dataset } \\
\text { of various web } \\
\text { pages. }\end{array}$ & $\begin{array}{l}\text { To predict } \\
\text { purchasing intention } \\
\text { for a virtual shopping } \\
\text { environment }\end{array}$ & $\begin{array}{l}\text { Real-time online } \\
\text { shopper behavior } \\
\text { analysis system }\end{array}$ & $\begin{array}{l}\text { Random forest, support } \\
\text { vector machines, } \\
\text { multilayer perceptron, } \\
\text { Long short-term } \\
\text { memory-based } \\
\text { recurrent neural } \\
\text { network. }\end{array}$ \\
\hline $\begin{array}{l}\text { Tahiri, N. et } \\
\text { al., } 2019\end{array}$ & $\begin{array}{l}\text { Grocery shopping } \\
\text { data from } \\
\text { MyGroceryTour.ca } \\
\text { website }\end{array}$ & $\begin{array}{l}\text { To predict the next } \\
\text { item of the basket } \\
\text { and next store of } \\
\text { purchase }\end{array}$ & $\begin{array}{l}\text { Modeled the habits of } \\
\text { the } \\
\text { MyGroceryTour.ca } \\
\text { site consumers }\end{array}$ & $\begin{array}{l}\text { Feedforward neural } \\
\text { network, recurrent } \\
\text { neural network }\end{array}$ \\
\hline $\begin{array}{l}\text { Gupta, V. et } \\
\text { al., } 2014\end{array}$ & $\begin{array}{l}\text { Posts from Quora } \\
\text { and Yahoo! } \\
\text { Answers website }\end{array}$ & $\begin{array}{l}\text { To predict the } \\
\text { purchase intention of } \\
\text { the user }\end{array}$ & $\begin{array}{l}\text { Proposed a feature } \\
\text { extraction method } \\
\text { based on n-gram }\end{array}$ & Linear SVM \\
\hline $\begin{array}{l}\text { Haque, R. et } \\
\text { al., } 2019\end{array}$ & $\begin{array}{l}\text { Tweets on Food } \\
\text { and Drink, Travel, } \\
\text { Education \& } \\
\text { Career, Goods \& } \\
\text { Services, Event \& } \\
\text { Activities, Trifle }\end{array}$ & $\begin{array}{l}\text { To identify purchase } \\
\text { intention }\end{array}$ & $\begin{array}{l}\text { Expanded seed } \\
\text { Intent-Indicators for } \\
\text { creating a semi- } \\
\text { automatic training set }\end{array}$ & $\begin{array}{l}\text { LSTM, Logistic } \\
\text { regression, Naïve } \\
\text { Bayes, Random forest, } \\
\text { and decision trees }\end{array}$ \\
\hline $\begin{array}{l}\text { Hamroun, } M . \\
\text { et al., } 2015\end{array}$ & $\begin{array}{l}\text { Electronics and } \\
\text { retail banking } \\
\text { datasets collected }\end{array}$ & $\begin{array}{l}\text { Intention analysis } \\
\text { based on consumer } \\
\text { reviews }\end{array}$ & $\begin{array}{l}\text { Customer Intentions } \\
\text { Ontology }\end{array}$ & $\begin{array}{l}\text { Lexico semantic } \\
\text { patterns-based } \\
\text { approach }\end{array}$ \\
\hline
\end{tabular}




\begin{tabular}{|c|c|c|c|c|}
\hline & $\begin{array}{l}\text { from popular } \\
\text { consumer review } \\
\text { sites }\end{array}$ & & & \\
\hline $\begin{array}{l}\text { Atouati, S. et } \\
\text { al., } 2020\end{array}$ & $\begin{array}{l}\text { Twitter data related } \\
\text { to British Airways }\end{array}$ & $\begin{array}{l}\text { To predict negative } \\
\text { purchase intent }\end{array}$ & NeighLoR & Logistic regression \\
\hline $\begin{array}{l}\text { Ding, X. et al., } \\
2015\end{array}$ & $\begin{array}{l}\text { User posts related } \\
\text { to kids and movies } \\
\text { from Sina Weibo }\end{array}$ & $\begin{array}{l}\text { To analyze user } \\
\text { intention of } \\
\text { consumption }\end{array}$ & $\begin{array}{l}\text { Consumption } \\
\text { Intention Mining } \\
\text { Model (CIMM) }\end{array}$ & $\begin{array}{l}\text { Convolutional neural } \\
\text { network }\end{array}$ \\
\hline
\end{tabular}

The above table lists every literature that has been discussed in this whole chapter. The purpose is to condense the knowledge gathered to extract the common tasks that were in focus previously. Undoubtedly, each literature focused on presenting distinct solutions to deliver personalized services by studying the user-product connections, however, the approach followed in each work varies in certain aspects. Some researchers focused on bricks and mortar stores whereas some concentrated on mining the patterns of online shoppers.

The designed models explained above are based on user and item attributes that attempt to explains consumer-product interaction methods. Techniques explained in related work such as regression and classification, Apriori algorithm has been used to derive recommendation models. By looking at the last column of the table, we conclude that most of the research works exploited neural networks or market basket analysis to build their proposed solution. However, according to Safitri, A., 2019, for the latter technique, it is necessary to have sufficient data records for each product, which directly affects the accuracy of the rules.

Moreover, the literature mentioned commonly drove their research by utilizing static historical data and overlooked the dynamics of the retail market. From the comparative description table, we can conclude that the literature above is using datasets 
of the current market that may limit the data volume and could also limit the horizon of the exploration.

However, the performance of these models directly relies on high interactive attributes of users and items which are often tough to obtain. To build a proper training model, it is important to have a larger training dataset that contains superior features and is cleaned according to the requirement of research. Besides the data volume issue, the index of the data has limited information that concerns the functionality of feature expansion. Moreover, the techniques employed in the literature review may create a problem to deploy in the environment where item features are susceptible to change according to the live context. Therefore, it brings the necessity of the prototype that operates on real-time data uploaded online by the users. 


\section{Chapter 4: Evaluation of Related work and Research description}

Throughout this chapter, we discuss the gap between the prior retail analysis reported and the industry's current requirements. Initially, we refer to the gaps and observations found in the related literature. The next section portrays the description of the research problems analyzed from the gaps. Then, we construct the research questions along with the methodologies to answer them. We wrap this chapter by discussing the objectives and research goals in detail.

\subsection{Gap Analysis}

In this section, we discuss the gaps we found from the comparison of related works. By analyzing the strengths and weaknesses of existing work in leveraging the retail data, we observed that many pieces of literature focused on prediction related to the demand and supply chain of the retail industry to organize the production, transportation, labor, and purchase force to increase sales and ultimately profit.

The gaps we find between the research papers in the technical domain of the retail market are that they tend to be more focused on building the models. During their studies, they often perform a thoroughly statistical analysis based on a special dataset and conclude results without much focusing on data pre-processing and feature scrutiny.

Moreover, from the findings above in related work review, the aspect of promotional planning (According to Hübner, A. H. et al., 2013, promotional planning is a method of maximizing the use of marketing techniques, tactics, services to advertise a product and service to increase demand, and achieving the defined targets) received less attention in the retail marketing literature, conceivably due to the risk involved in the 
applied process. In the area of promotional marketing, the exactness of the applied model can significantly encourage lower storage costs and increase client satisfaction. Though, the risk of the wrong estimation can lead to costly losses either by excess or disruption.

We believe that the series of recommendation framework built on the ground of new feature expansion can lead to alleviating the disparity in the research field of retail promotional planning. Furthermore, the new features extracted combined with existed common technical indices will benefit the well-tested models as well. This research work will emphasize the evaluation of some of the most relevant algorithms used for forecasting consumer demand and investigating their purchase intent by predicting the probability of product to be repurchased and hence, comparing their performance.

There have been substantial attempts in the field of intention detection. Of the major disparity to tackle in the field of purchase intention (PI) detection using machine learning algorithms, is to define purchase intent labels similar to the sentiment analysis technique. Most of the related works developed a classifier for the problem that detects PI or non-PI class. However, the PI class needs further investigation to extract descriptive intent labels such as Positive Purchase intent, negative purchase intent, or unknown. The extracted knowledge could then be utilized to comprehend better marketing strategies and promotional schemes.

\subsection{Research problem description}

The E-commerce market is increasing rapidly nowadays, with the support of superior internet infrastructure. The rising number of online stores in the market competes for attracting customers. Therefore, online shopping platforms must have an application that can classify the positive purchasing intentions of the customers by identifying 
customer's historical buying behavior and social media interactions with the products. Our research aims to draw the consumer's eyes on the potential products that will eventually lead to an increase in sales and revenue. In the literature, as some of it discussed in the previous chapter, there are several foremost bases for article suggestion problem. One foundation is personalization, undergirded by measures that are user-specific. Another is the correlation amongst all the Instacart products. Our focus in this work is to provide a framework that integrates both of the above-mentioned foundations.

To build a prediction model on Instacart's historical as well as social media data, initially, it is necessary to understand the problem. In the dataset, we obtained a relational database of previous purchases done by the customers. We aim to investigate the relative relationship between the individual user data and product data. Explicitly, we want to find those Instacart users who might repurchase the product in their next purchase cycle. To incentivize those customers, various promotional activities could be put together to increase sales and the ultimate profit. Therefore, we focus to build a machine learning model that assigns probabilities to the products that could be repurchased by a user of Instacart based on the customer's historical purchase data. The target product repurchasing probabilities would be utilized to comprehend the propensity of the online users and their purchase intentions to consume the product. The research findings could be then used as an input to leverage the sales by providing promotional discounts to the consumer to tend to buy the product that has maximum probabilities and effective user propensity.

As described in chapter 2, data revealed by Instacart contains the information of $200 \mathrm{k}$ distinct users with the number of orders that vary from 4 to 100 . Each order is discretely indicated as prior, train, or test order under eval_set feature. Orders marked as 
Prior describes the past behavior of a specific user whereas test and train orders are considered to predict the future behavior of the users. Therefore, the decisive goal is to predict which prior orders of a user could be the user's next order. The table below is a pictorial representation of the problem statement. Each user has several previous orders $(\mathrm{O} 1, \mathrm{O} 2, \mathrm{O} 3$, so on) which are termed as prior orders of the user. Orders in red denote the upcoming order $(\mathrm{PO})$ of the user which could be either considered as test or train order.

Table 7. Instacart Problem description table

\begin{tabular}{|c|c|c|c|c|c|c|c|c|c|}
\hline & \multicolumn{9}{|c|}{ Order Number } \\
\hline & 1 & 2 & 3 & 4 & 5 & 6 & 7 & ........ & Nth \\
\hline User A & 01 & $\mathrm{O} 2$ & $\mathrm{O} 3$ & 04 & PO & & & & \\
\hline User B & 01 & 02 & 03 & 04 & 05 & 06 & PO & & \\
\hline User C & 01 & $\mathrm{O} 2$ & 03 & 04 & 05 & 06 & 07 & 0 & PO \\
\hline User D & 01 & 02 & 03 & 04 & 05 & PO & & & \\
\hline
\end{tabular}

Hence, each user in the training data frame has a set of products that were purchased in prior orders and order_id of each upcoming order. The aim is to predict the products that will be purchased by the user in the future. In the nutshell, the research work is a classification problem as we need to predict whether a specific user will repurchase the product or not (1 or 0$)$.

\subsection{Research questions}

We address these three research questions in different aspects:

\section{RQ1. How does feature expansion benefit model prediction accuracy?}

The first research question is about feature expansion. We would like to know the impact of feature extension on the performance of prediction models.

The plentiful previously done work in this field determines the dynamic purchase behavior of the users, which makes the prediction task notoriously difficult. Hence, to 
sort the high level of noise embedded in retail data, feature engineering could be used as an optimization module.

\section{RQ2. How to predict whether a user is inclined to buy a product or not?}

The second research question is about technical design selection, i.e., what could be the best technique to model our data efficiently?

Based on previous works, researchers are focused on determining products that are high in demand. While we segregate the problem into predicting the products that will be re-purchased in the next shopping trip and then exploiting the highest probability products to examine the user's intention of consumption. Our approach is to decompose the complex problem into sub-problems which have fewer dependencies that will result in a one-by-one problem handling approach. The final step will include the compilation of resolutions into an integrated model as an aiding system for the promotional marketing of products.

\section{RQ3. How to utilize the prediction of product repurchase probability and user's intention detection in a real-life scenario?}

The third research question is evaluating the scope of findings we extracted from our model design.

Different from previous work, besides the common evaluation of the model to extract information about personalized products and user intentions to purchase, our evaluation model 1 will emphasize the effectiveness of newly added features to determine the product selling probabilities, while model 2 will highlight the propensity level of the online consumers. The significance of our final model 3 will spot the 
consumer's purchase intention by gauging online reviews. The raw data from Instacart needs to be processed into usable features and then we will combine the features with other technical indices to assess the features with a high impact. Eventually, how to appropriately convert the findings into shaping the promotional strategies to increase the attraction of consumers is another hidden research question.

\subsection{Research Objective}

The central objective of this study is to investigate consumer's perceptions and intentions to purchase groceries via comprehending the historical purchase transactions and online reviews. The goal is to understand the relationship between perceptual data variables and their influence on product merchandising. The research methodology pursued to answer the research questions are:

\section{RO1. Feature Expansion to create structured data from unstructured data.}

The first research objective is to engineer new features based on the given Instacart data to create significant variables for evaluation.

As stated by Domingos, P. 2012, Feature engineering is an essential process of extracting new features from the raw dataset using data mining techniques, which in response determines the failure or success of a machine learning algorithm. In this process, we will manually create some new features that are extracted from raw data using relational joins and calculations on the data. This will make the training set of the model more comprehensive and stronger to capture more relevant information and patterns. 


\section{RO2. Build techniques to effectively predict the purchase probability of products and}

user's purchase intention by analyzing the performance of different machine learning and deep learning models.

The second research objective is purely a technical step taken towards accessing the classification metrics results of the machine learning algorithms on our dataset.

The objective is to apply different machine learning algorithms to the structured data. By the analysis of feature importance and best model accuracy, we aim to design a solution that will enhance the prediction performance and rule the overall evaluation matrices such as time complexity, space complexity.

\section{RO3. Use case based on product repurchasing probability and user's intention prediction to expand the growth of the retail market.}

This research objective is of the use case of our proposed solution i.e. how to implement the proposed solution as an application.

The major purpose of this use case is to build an easy-to-access front end for the retailers that retain the consistency of our system components that can be compiled as an application anytime. The interface will provide a time-efficient procedure to evaluate the behavior of the customer and could help retailers in strategizing promotional offers that could be provided in order to gain revenue.

The overall goal of this thesis is to describe a user-friendly web interface for retailers that employs scalable data to analyze the different products in the market and how they are interrelated to consumer's needs and shopping patterns. We exploit the user-item relations to derive market strategies that can benefit retailers to leverage the sales and cut off the products if not generating revenue. 


\section{Chapter 5: Experiments using various machine learning techniques}

This experimental section was exclusively built using only classic Machine Learning (CML) methods, such as Random Forest, AdaBoost Decision Tree, and Support Vector Machine. Open source Scikit-learn (http://scikit-learn.org, CPU of 8GB RAM for training and prediction) ML python library was used for building, tuning, and validating all the machine learning models. The developed model consists of a random splitting of the input dataset into training $(80 \%)$ and test $(20 \%)$ datasets, while maintaining equal proportions of class ratios in each split.

The high-level architecture of our experiments could be separated into five parts. First is to import the CSV files of our experimental data and reshape the data frames in an effective way such as transforming the characters into categorical variables. Second, we investigate the data to perform feature extensions. This step includes identifying and calculating new features from the initial variables of the dataset provided by Instacart. The third step includes the distinction of training and testing data to create the prediction model and train it. And the last part which is the main contribution of our work is to apply the model to predict the 'repurchased' variable (target) for the test dataset.

\subsection{Data Acquisition}

For computation following software modules were installed: scikit-learn 0.18.1, Python 3.5.2, Anaconda 4.2.0 (64-bit), Keras 1.2.1, Tensorflow 0.12.1, Jupyter Notebook 4.3.1. Python libraries were imported that are used for performing data manipulation (pandas), splitting data in train-test (sklearn.model_selection), to perform random forest classification (sklearn.ensemble), to calculate the accuracy of the prediction 
(sklearn.metrics). All the data tables were fed to the IDE via reading command (which is included in pandas) to store them in an environment for manipulation.

\subsection{Data Processing and Feature Expansion}

This part is the elaboration of the detailed data processing from raw data reshaping to feature extension. We first expand our raw data fields to generate new features. Then we will feed the data into the machine learning models to get the prediction results. For the experimental evaluation, we will not only apply machine learning methods on raw data but also apply the methods on extended features to evaluate which has relatively better prediction accuracy.

We transform the raw data in order to facilitate the model design and analysis. We altered the character variables into categorical to create the model such as aisle name, department name, and eval_set by assigning a fixed number of different values to each categorical variable. To provide a meaningful dataset to the model, we create a new data frame by joining the orders data and prior records from eval_set of order_products data directing at the target label reordered. The new table named prior_op contains information about all customers and their orders along with each product that was bought in each order.

\subsubsection{Feature Expansion}

The provided input data of prior orders is used to calculate the predictor variables which will describe the behavior of the users, characteristics of the products, and user activities towards one or multiple products. We will train the algorithm based on the new variables to respond to the target variable. The following table explains the features derived from raw data to facilitate the prediction: 
Table 8. Extended features description table

\begin{tabular}{|c|c|c|c|}
\hline Feature name & Description & Feature processing & Pseudo-code \\
\hline \multicolumn{4}{|c|}{ User-based variables } \\
\hline user_orders_max & $\begin{array}{l}\text { Number of orders } \\
\text { per customer }\end{array}$ & $\begin{array}{l}\text { The total number of orders placed } \\
\text { by each customer is calculated by } \\
\text { making a group for each user with } \\
\text { the help of .groupby function }\end{array}$ & $\begin{array}{l}\text { user }= \\
\text { op.groupby('user_id')['order_n } \\
\text { umber'].max().to_frame('user_ } \\
\text { orders max') }\end{array}$ \\
\hline users_reorder & $\begin{array}{l}\text { Frequency of } \\
\text { repurchasing } \\
\text { products }\end{array}$ & $\begin{array}{l}\text { The frequency is calculated by } \\
\text { taking the mean of all the times a } \\
\text { user has repurchased products } \\
\text { (reordered = 1) with respect to the } \\
\text { total number of purchased } \\
\text { products for each user (reordered } \\
1+\text { reordered } 0 \text { ) }\end{array}$ & $\begin{array}{l}\text { users_reorder }= \\
\text { op.groupby('user_id')['reorder } \\
\text { ed'].mean().to_frame('users_re } \\
\text { ordered') }\end{array}$ \\
\hline $\begin{array}{l}\text { orders_since_first_ } \\
\text { order }\end{array}$ & $\begin{array}{l}\text { Number of orders } \\
\text { placed by each } \\
\text { customer since the } \\
\text { first order is placed }\end{array}$ & $\begin{array}{l}\text { It is calculated by adding } 1 \text { to the } \\
\text { difference between } \\
\text { user_order_max and } \\
\text { first_order_num }\end{array}$ & $\begin{array}{l}\text { span['orders_since_first_order' } \\
\text { ] = span. } u \text { ser_order_max - } \\
\text { span.first_order_num }+1\end{array}$ \\
\hline \multicolumn{4}{|c|}{ Product-based variables } \\
\hline purchase_count & $\begin{array}{l}\text { Number of } \\
\text { purchases done for } \\
\text { each product from } \\
\text { all customers }\end{array}$ & $\begin{array}{l}\text { The purchase count of each } \\
\text { product is calculated by using } \\
\text { groupby function on user id and } \\
\text { product id }\end{array}$ & $\begin{array}{l}\text { purchase_count }= \\
\text { op.groupby('product_id')['orde } \\
\text { r_id'].count().to_frame('purcha } \\
\text { se_count') }\end{array}$ \\
\hline reorder_prob & $\begin{array}{l}\text { Repurchasing } \\
\text { probability of each } \\
\text { product }\end{array}$ & $\begin{array}{l}\text { The probability of repurchasing } \\
\text { certain products is calculated by } \\
\text { taking the mean of the number of } \\
\text { repurchases with respect to the } \\
\text { total number of orders }\end{array}$ & $\begin{array}{l}\text { reorder_prob }= \\
\text { op.groupby('product_id').filter( } \\
\text { lambda } x: \text { x.shape[0] }>40) \\
* \text { shape[0] returns the number } \\
\text { of rows of the data frame. }\end{array}$ \\
\hline first_order_num & $\begin{array}{l}\text { Order number } \\
\text { where the user } \\
\text { bought a specific } \\
\text { product for the first } \\
\text { time }\end{array}$ & $\begin{array}{l}\text { Each user id is grouped with } \\
\text { product id and a minimum of } \\
\text { order_number is retrieved for } \\
\text { each group }\end{array}$ & $\begin{array}{l}\text { first_order_num = } \\
\text { op.groupby(['user_id', } \\
\text { 'product_id'])['order_number'] } \\
\text {.min().to_frame('first_order_nu } \\
\text { m') }\end{array}$ \\
\hline $\begin{array}{l}\text { reverse_order_nu } \\
\text { m }\end{array}$ & $\begin{array}{l}\text { This feature defined } \\
\text { the last order as } 1 \text {, } \\
\text { second last as } 2 \text { and } \\
\text { so on }\end{array}$ & $\begin{array}{l}\text { Calculated by subtracting } \\
\text { order_number of each order from } \\
\text { user_orders_max plus } 1 \text { to adjust } \\
\text { the index. }\end{array}$ & $\begin{array}{l}\text { span['reverse_order_num'] }= \\
\text { span. user_order_max }- \\
\text { span.order_number }+1\end{array}$ \\
\hline \multicolumn{4}{|c|}{ User \& product-based variables } \\
\hline up_count & $\begin{array}{l}\text { Number of times } \\
\text { each user bought } \\
\text { the product }\end{array}$ & $\begin{array}{l}\text { It is calculated by combining the } \\
\text { users and products and then } \\
\text { aggregating the number of times a } \\
\text { product is bought by the user } \\
\text { using .count () function }\end{array}$ & $\begin{array}{l}\text { up_count }= \\
\text { op.groupby(['user_id', } \\
\text { 'product_id']])['order_id'].coun } \\
\text { t().to_frame('up_total_bought') }\end{array}$ \\
\hline up_reorder & $\begin{array}{l}\text { Frequency of } \\
\text { repurchasing after } \\
\text { the first purchase of } \\
\text { the product }\end{array}$ & $\begin{array}{l}\text { It is calculated by taking the ratio } \\
\text { of up_count with respect to } \\
\text { orders_since_first_order for each } \\
\text { user and product }\end{array}$ & $\begin{array}{l}\text { up_ratio['up_reorder'] }= \\
\text { up_ratio.up_count / } \\
\text { uxp_ratio.orders_since_first_or } \\
\text { der }\end{array}$ \\
\hline last_5orders & $\begin{array}{l}\text { Number of times a } \\
\text { customer bought a } \\
\text { product on its last } 5 \\
\text { orders }\end{array}$ & $\begin{array}{l}\text { By assigning reverse_order num } \\
<=5 \text {, we calculated the last five } \\
\text { orders of each user }\end{array}$ & $\begin{array}{l}\text { Span[last_5orders] }= \\
\text { span['reverse_order_num'] } \\
<=5\end{array}$ \\
\hline
\end{tabular}

All the newly constructed feature variables (left join combination of user \& product variables with user-based and product-based variables) are then merged with primary keys 
i.e. user_id and product_id in order to create our final data frame (finaldata) to feed the model.

\subsubsection{Final data split}

To split the final data frame created in the previous section into two datasets (train and test), we added two more columns from the initial orders table i.e. eval_set and order_id with respect to $u s e r \_i d$ which acts as matching key between orders and finaldata data frames. The column eval_set will define the type or the user and order_id will serve as information of future order.

To separate the training and testing data we filtered the eval_set feature. While preparing the training dataset, we added the reordered column (target variable) for the learning process of our model. All the missing/unavailable values in the reordered column were replaced by 0 to bring into a structure for machine learning. The test data frame is prepared similarly by filtering the test users in eval_set feature and reordered feature was excluded as it is the label of prediction. To partition the data into training and testing sets, we call the model_selection module from the sklearn library (Training and testing data splitting module from Scikit-learn library, 2007).

\subsubsection{Handling Imbalanced data}

Chawla, N. V. et al., 2002 introduced a novel technique "Synthetic Minority oversampling technique" (SMOTE) of constructing new classifiers using Euclidean distance measure on the nearest neighbor instances. Due to the imbalanced number of binary classification cases in the final dataset, we generated new instances from existing minority data records in a balanced way. By correcting the bias in the model, SMOTE also reduces 
the chances of data overfitting. We have adapted the code from "ML | Handling Imbalanced data with SMOTE" at geeksforgeeks.com (Tyagi, K., 2020) to implement the handling of the class imbalance problem.

\subsection{Model evaluation and analysis}

After the preprocessing step, Data Mining algorithms are performed on the original dataset as well as on the structured dataset to find the best one in the prediction of the product to be repurchased by comparing the accuracies between them.

To ensure the best performance of the prediction model, we will investigate the raw data first, and then the trained model will be applied to the structured dataset. There are many features in the primal data, if we involve al the features into our consideration, it will not only drastically increase the computational complexity but will also cause side effects (for example, overfitting) to the performance accuracy. Hence for original data evaluation, we merged the orders data frame with product_id, reordered, and add_to_cart column features.

The training set is further divided into a validation data set to ensure the optimal accuracy score to achieve the finest threshold for prediction. For the model evaluation of the historical dataset, we balanced the data between target binary classification of the data records (repurchase or not), hence, we consider accuracy as dominant metrics in the classification report.

\subsubsection{Random Forest Classifier}

Random forest (Breiman, L., 2001) is an ensemble of many decision trees. It generates many classification trees by collecting bootstrapping samples from original data 
that spits our class prediction. The class with the most votes is chosen for our model's prediction. The algorithm draws $\mathrm{n}$ number of bootstrap samples from $\mathrm{n}$ training sets. For each of $\mathrm{n}$ samples, the algorithm tries to try $\mathrm{m}$ samples of features and chooses the best split from those variables. Bagging is termed as a special case where all the variable predictors are used. The final prediction is calculated by aggregating the predictions of the $\mathrm{n}$ trees (i.e., For classification majority votes are considered, the average for regression) (Svetnik, V. et al., 2003; Liaw, A., \& Wiener, M., 2002).

This experiment was implemented based on the code adapted from the "Random forest algorithm with Python and scikit learn" article on stackabuse.com (Malik, U., 2020) and "Random forest in python" on towardsdatascience.com (Koehrsen, W., 2017). We adjust the threshold value to attain the best accuracy and F1 score for the model.

\section{a) Outputs:}

After applying the random forest classification model on both raw data and structured data frame, we compare the output feature set. We used two different approaches to evaluate model effectiveness. The first method is the Receiver operating characteristic curve (ROC) which plots the true positive rate (TPR) against false positive rate (FPR) (Drummond, C., \& Holte, R. C., 2000). ROC is a graphical representation that demonstrates the diagnostic abilities of the classifier. The classifier that bends closer to the top-left corner is considered as best performing. The area of the curve determines the overall diagnostic ability of the classification model. A perfect model with $100 \%$ accuracy will have an area of 1.00 under the curve (Narkhede, S., 2018)

Another method of quality evaluation is through a normalized confusion matrix which gives the summary of prediction results with count values. A binary confusion 
matrix is a table of two rows and two columns that presents the number of true-positive (TP), true-negative TN), false-positive (FP), and false-negative (FN) (Sharma, A., 2020).

The accuracy of the classifier is visualized by comparing the actual and predicted classes.

The output indices are illustrated in the table below:

Table 9. Model output description table

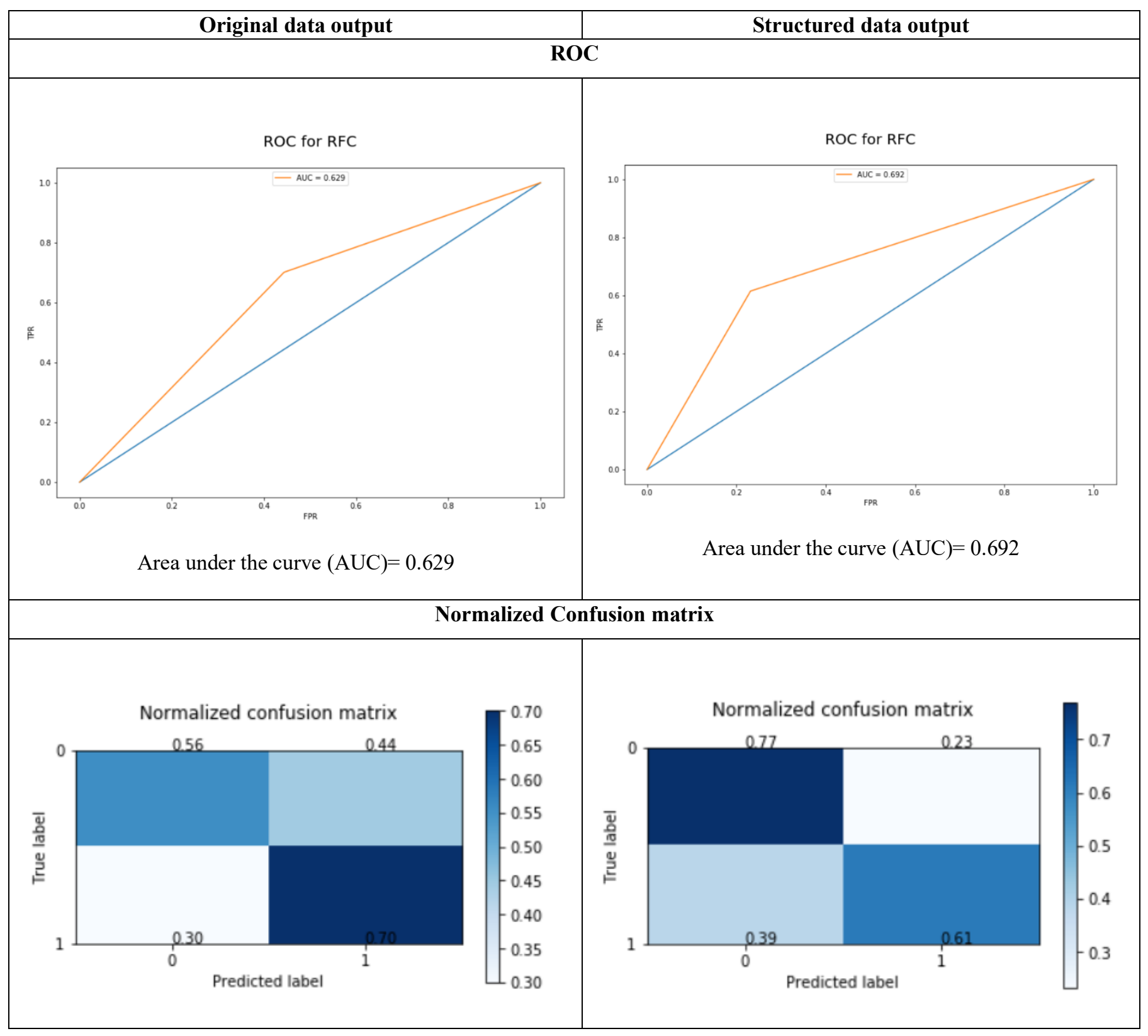


Table 9 shows the ROC curve and confusion matrix obtained from the Random forest classification. In the confusion matrix, the predicted class labels are listed on the xaxis and along the y-axis are the true class labels. The first diagonal is termed as correct classifications of the product repurchase, whereas all the other entries show misclassifications. The true classification rate of the structured data model is better than the raw data model.

Therefore, our first experiment results substantiate our hypothesis, that extracted features yields marginally better results than the unprocessed data features.

\section{b) Functional analysis:}

This section creates a baseline for the classification report for the comparison of the prediction model. Table 10 records the detailed evaluated results of the random forest classifier and demonstrates the training efficiency of the model.

Table 10. Classification report

\begin{tabular}{|c|c|c|}
\hline & Original Data Report & Structured Data Report \\
\hline Accuracy (\%) & $64.3 \%$ & $75.3 \%$ \\
\hline Precision & 0.70 & 0.22 \\
\hline F1 Score & 0.70 & 0.32 \\
\hline Sensitivity & 0.70 & 0.61 \\
\hline Specificity & 0.55 & 0.76 \\
\hline AUC & 0.62 & 0.69 \\
\hline FPR (fall-out) & 0.44 & 0.23 \\
\hline FNR (miss rate) & 0.29 & 0.38 \\
\hline
\end{tabular}

Table 10 also indicates the overall prediction accuracy which is drastically improved by the feature expansions. We will also initiate a discussion upon the evaluation result in the conclusion section 5.4. 


\subsubsection{Extreme gradient Boosting (XGBoost) on Random forest classifier}

XGBoost stands for extreme gradient boosting decision trees (Chen, T., \& Guestrin, C., 2016). It is an implementation of gradient boost ( $\mathrm{gbm})$ decision trees for speed and performance. In the gradient boosting algorithm, the new models are created by predicting the residuals or errors of prior models and then added together to make the final prediction. It uses a gradient descent algorithm to decrease the loss when adding new models (Chen, T., \& Guestrin, C., 2016).

It is a novel sparsity aware algorithm that operates sparse input data for approximate learning of both tree booster and linear booster. It also supports customized objective functions and evaluations including regression, classification. User can define their objective functions as well. We defined a random forest classifier as a weak base estimation model which is further boosted by fitting XGBoost classifier. The model computes the prediction results by adding the weights and multiplying the predictions of each tree. The ensemble model consisting of XGBoost is capable to do parallel computation and hence it is faster than the Classic AdaBoost algorithm (Jiang, Y. et al., 2019).

The code for this experiment was adapted from the "Using XGBoost in Python" article on datacamp.com (Pathak, M., 2020) and "A simple XGBoost tutorial using Iris dataset" on kdnuggets.com (Zarina, I., 2020).

\section{a) Outputs:}

The output indices are illustrated in the table below: 
Table 11. Model output description table

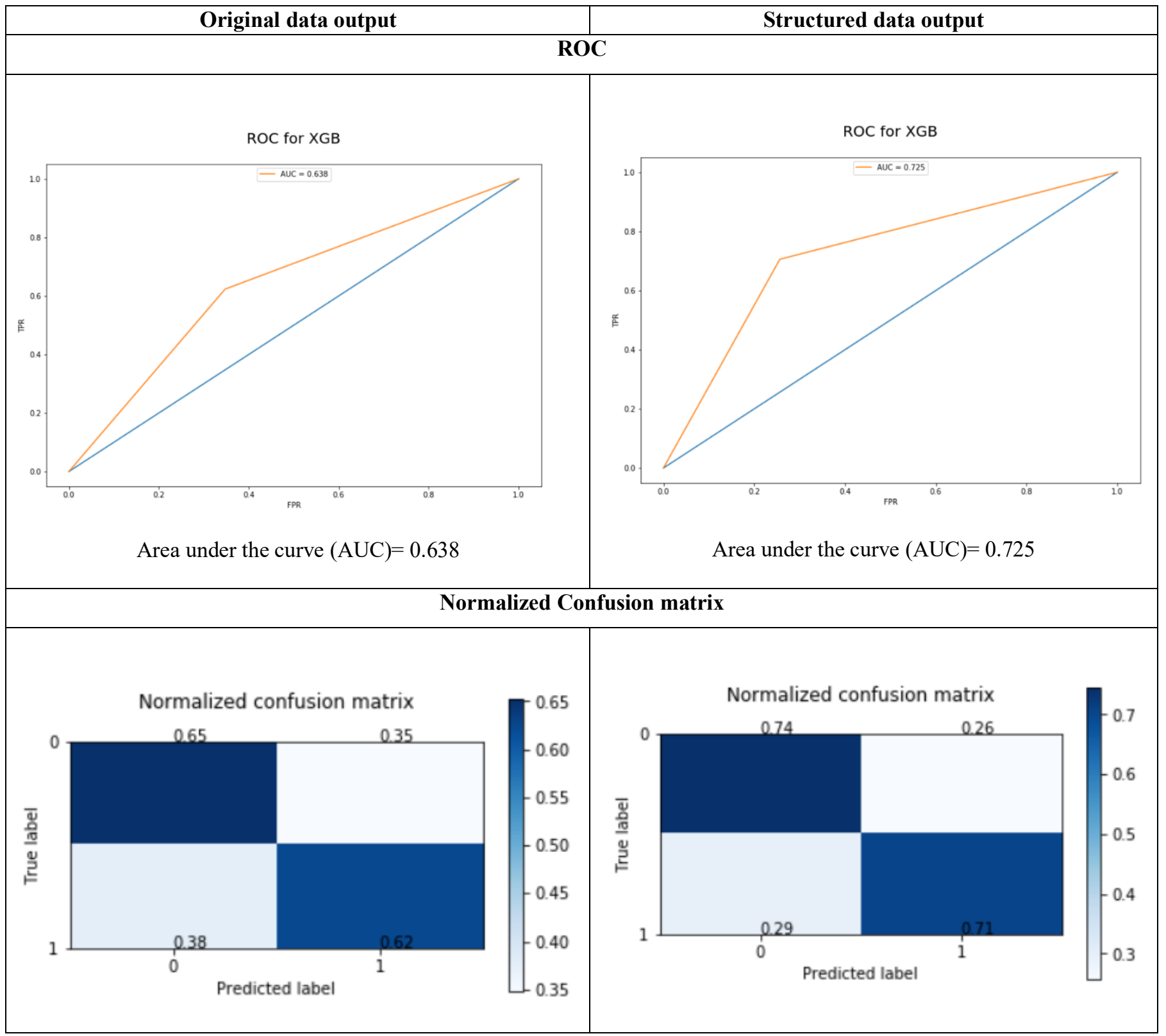

In comparison to the random forest classifier, XGBoost exhibits slightly better classification results for true labels and improved area under the ROC curve.

\section{b) Functional analysis:}

Table 12 records the detailed evaluated results of the XGBoost classifier and demonstrates how the quality of features affects the model training efficiency. 
Table 12. Classification report

\begin{tabular}{|c|c|c|}
\hline & Original Data Report & Structured Data Report \\
\hline Accuracy (\%) & $64.3 \%$ & $74.1 \%$ \\
\hline Precision & 0.72 & 0.22 \\
\hline F1 Score & 0.67 & 0.34 \\
\hline Sensitivity & 0.62 & 0.70 \\
\hline Specificity & 0.65 & 0.74 \\
\hline AUC & 0.63 & 0.72 \\
\hline FPR (fall-out) & 0.34 & 0.25 \\
\hline FNR (miss rate) & 0.37 & 0.29 \\
\hline
\end{tabular}

The results of ensemble XGBoost classification over the random forest learner base verifies that data prepared with extracted features perform better than raw data features.

\subsubsection{CatBoost on Random forest Classifier}

CatBoost is based on the gradient boosting technique. It is a new machine learning technique developed by Yandex that surpasses many existing boosting algorithms like XGBoost, Light GBM (CatBoost, 2014). CatBoost enables to handle of the categorical data by dividing the given dataset into random permutations and applies ordered boosting on those random permutations which help to decrease the risk of overfitting the model (Swalin, A., 2020). Analogous to XGBoost in section 5.3.2, we used the random forest classifier as a weak learner base which is further boosted using the CatBoost technique.

This experiment was implemented by adapting the code from the "Python-UsageExample" article on CatBoost.ai (Gulin, A. et al., 2020) and "CatBoost with gridsearchcv" on Kaggle.com (Kazachok, M., 2020).

\section{a) Outputs:}

The outputs are illustrated below in table 13: 
Table 13. Model output description table

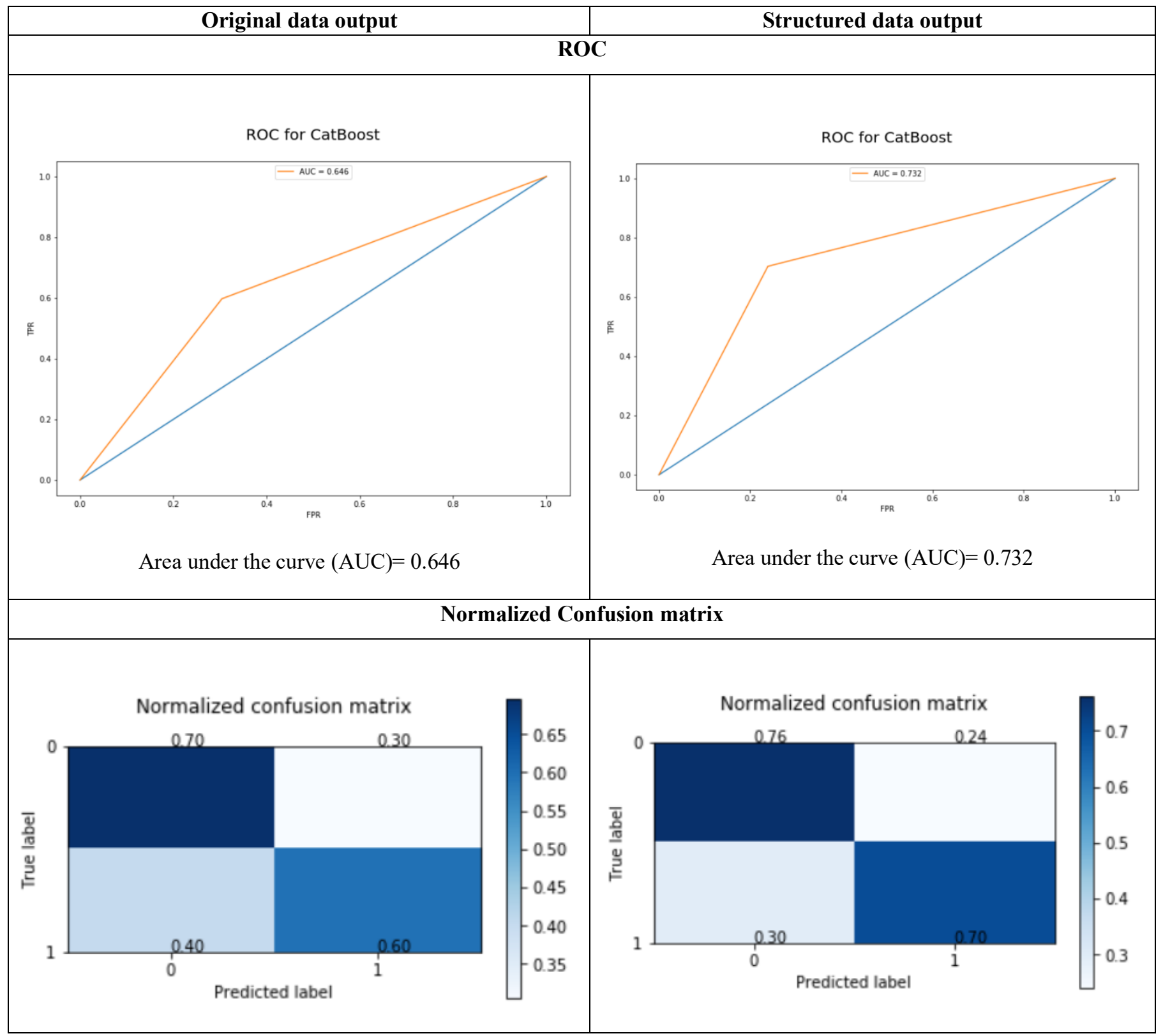

In comparison to XGBoost, CatBoost classifier performs marginally better in terms of area under the curve and true label categorization. This ensemble experiment also backs up our hypothesis that extracted features from the original data improves the overall performance of the model. 


\section{b) Functional analysis:}

Table 14 records the detailed evaluated results of the Catboost classifier and validates improved the model efficiency when trained on extracted features.

Table 14. Classification report

\begin{tabular}{|c|c|c|}
\hline & Original Data Report & Structured Data Report \\
\hline Accuracy (\%) & $66.2 \%$ & $75.5 \%$ \\
\hline Precision & 0.69 & 0.54 \\
\hline F1 Score & 0.73 & 0.36 \\
\hline Sensitivity & 0.78 & 0.70 \\
\hline Specificity & 0.48 & 0.76 \\
\hline AUC & 0.63 & 0.73 \\
\hline FPR (fall-out) & 0.51 & 0.23 \\
\hline FNR (miss rate) & 0.21 & 0.29 \\
\hline
\end{tabular}

\subsubsection{Artificial Neural Network (ANN)}

Artificial Neural Network is a technique of information processing motivated by the biological nervous system (Cherkassky, V. et al., 2006). It uses various layers of mathematical processing which operates on nodes that are highly interconnected using weights (Chauhan, N. S., 2020). A node combines input with these connection weights in order to perform the learning task (Saha, A. K. et al., 2002). According to Prasad, R. et al., 2012, ANN is an efficient machine learning algorithm that can be optimized using various coefficients and are useful for a system that is known for high error tolerance.

The code for this experiment is adapted from "How to build your own Neural network in python" article on towardsdatascienc.com (James Loy, 2018) and "Simple neural network" on kdnuggets.com (Garbade, Dr. M. J., 2018). 


\section{a) Outputs:}

Table 15. Model output description table

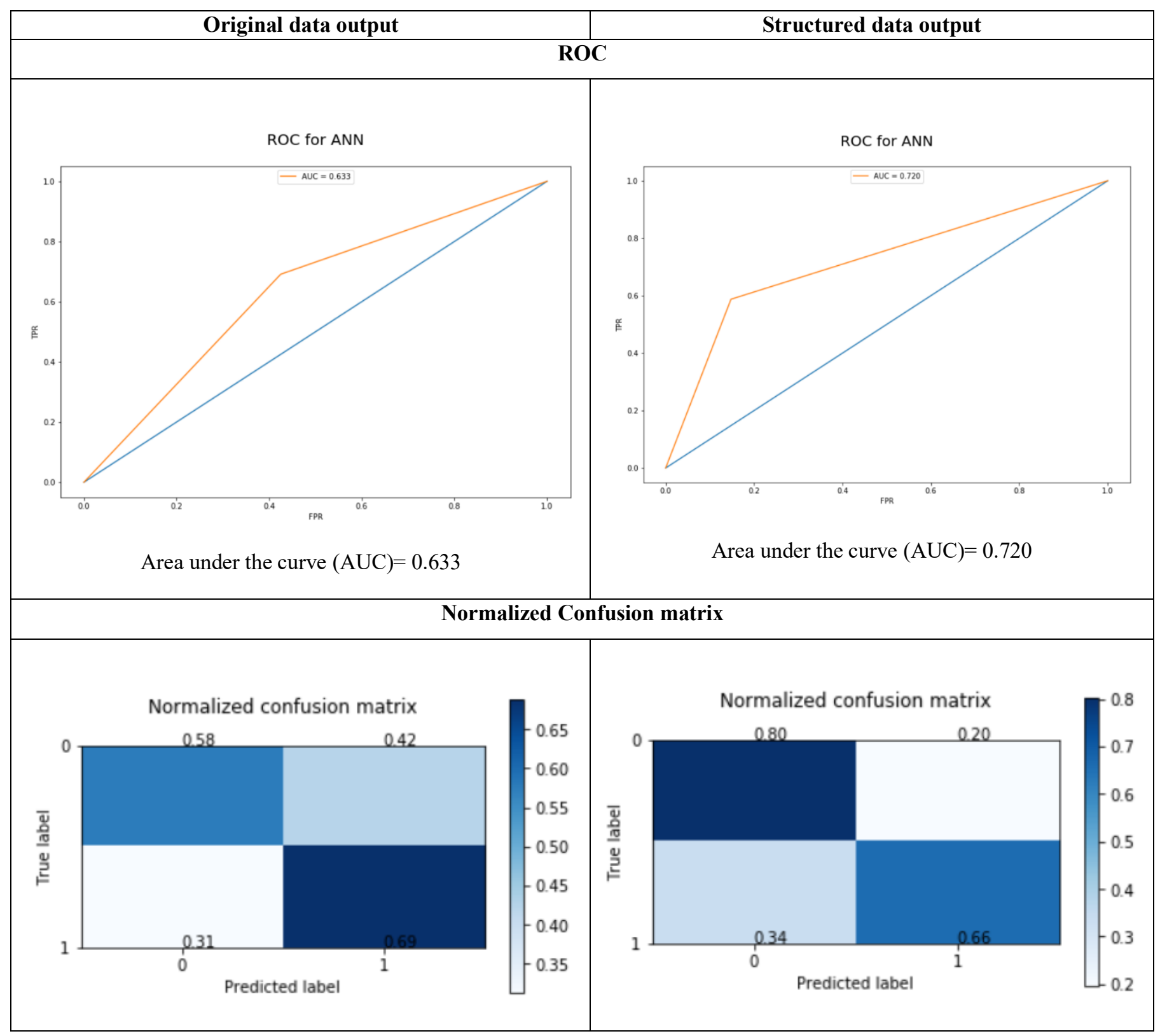

Clearly, the increased number of true classifiers and superior area under the curve for the structured data model ranks better than original data features. 


\section{b) Functional analysis:}

Table 16 records the detailed evaluated results of ANN and demonstrates how the number of features affects the model training efficiency.

Table 16. Classification report

\begin{tabular}{|c|c|c|}
\hline & Original Data Report & Structured Data Report \\
\hline Accuracy (\%) & $64.3 \%$ & $82.3 \%$ \\
\hline Precision & 0.70 & 0.73 \\
\hline F1 Score & 0.69 & 0.39 \\
\hline Sensitivity & 0.68 & 0.58 \\
\hline Specificity & 0.57 & 0.84 \\
\hline AUC & 0.63 & 0.72 \\
\hline FPR (fall-out) & 0.42 & 0.15 \\
\hline FNR (miss rate) & 0.31 & 0.41 \\
\hline
\end{tabular}

\subsubsection{Convolutional Neural Network (CNN)}

A convolutional neural network is a category of deep artificial neural networks. $\mathrm{CNN}$ is a feed-forward model as information flows right through the model with no output connections back to feed into the model itself. Each neuron of the first layer is connected to all neurons of the next (Brownlee, J., 2019; Sharma, A., 2017). A conventional CNN contains four types of layers: convolutional, activation, pooling, and fully connected (or dense) layers. According to the Convolutional neural network, 2020, the network contains an input and an output layer separated by multiple hidden layers that convolve the input and pass it to the next layer. As stated by Howard, A. G., 2013, The training of CNNs is done analogous to that of other ANNs which includes minimizing loss functions using gradient descent-based methods along with backpropagation of the error.

The code to implement this experiment is adapted from "Binary Classification Tutorial with the Keras Deep Learning Library" on machinelearningmastery.com 
(Brownlee, J., 2019) and "Convolutional Neural Networks in Python with Keras" on datacamp.com (Sharma, A., 2017).

a) Outputs:

Table 17. Model output description table

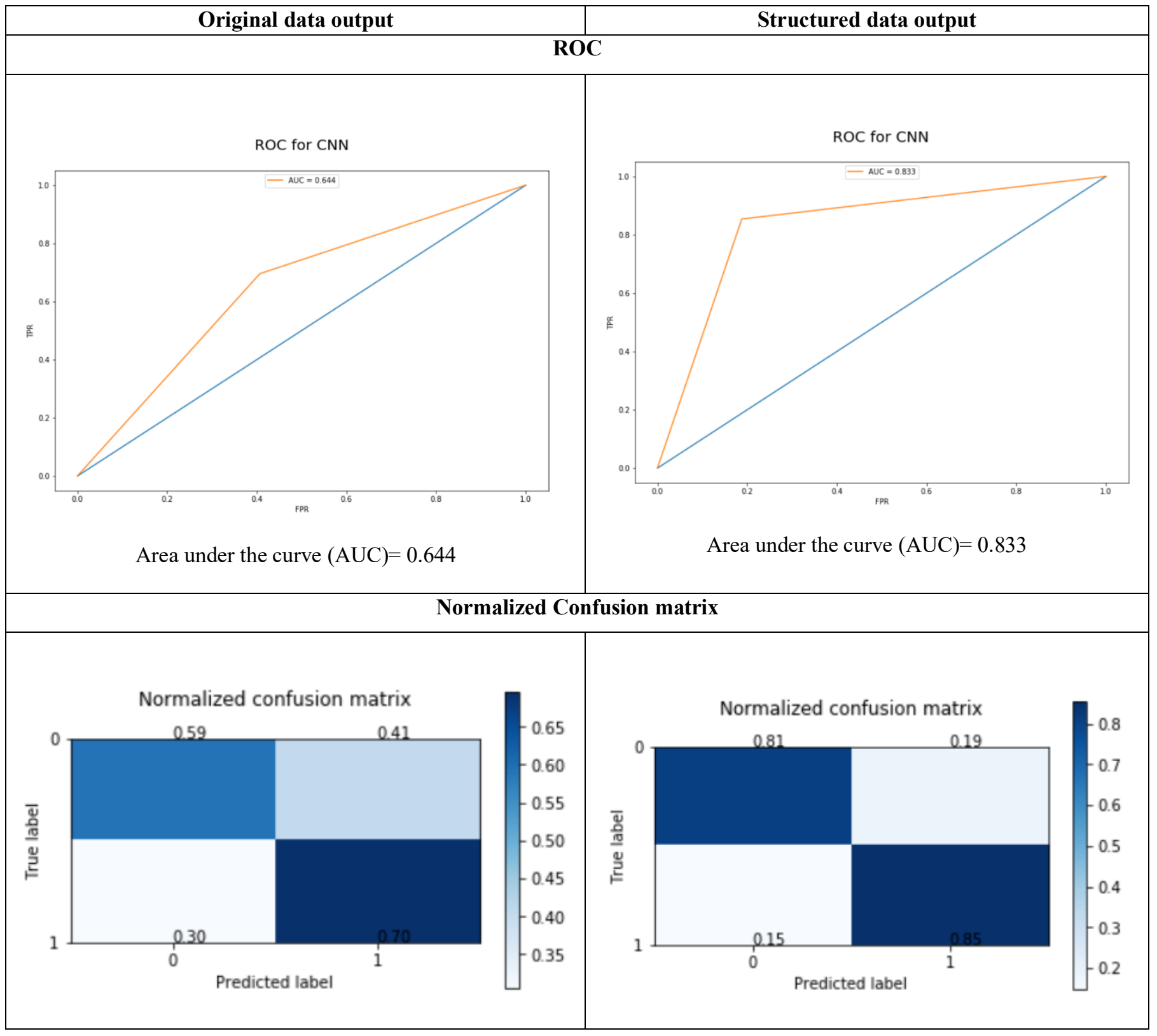




\section{b) Functional analysis:}

Table 18 records the detailed evaluated results of $\mathrm{CNN}$ and demonstrates how the designed features affect the model training efficiency. This model design has the highest average accuracy of $83.6 \%$ as compared to all other models.

Table 18. Classification report

\begin{tabular}{|c|c|c|}
\hline & Original Data Report & Structured Data Report \\
\hline Accuracy (\%) & $65.4 \%$ & $83.6 \%$ \\
\hline Precision & 0.71 & 0.81 \\
\hline F1 Score & 0.70 & 0.41 \\
\hline Sensitivity & 0.69 & 0.85 \\
\hline Specificity & 0.59 & 0.81 \\
\hline AUC & 0.64 & 0.83 \\
\hline FPR (fall-out) & 0.40 & 0.18 \\
\hline FNR (miss rate) & 0.30 & 0.14 \\
\hline
\end{tabular}

The detailed comparison discussion of all the designed experiment results will be initiated in the following section.

\subsection{Model performance Comparison}

In this part, we compared our ensemble experiment outcomes. From the results of the previous subsection, we can see that when predicting the repurchasing probability of the Instacart products, the best result is achieved by manually processed features than original features, which proves that the feature expansion method is useful for optimizing the model.

Regarding the performance attained by the different models built with different predictors, the following Figure 14 illustrates the effect of feature engineering on the accuracy classification report computed upon five different ensemble machine learning experiments. The bar graph below indicates the algorithm names on the $\mathrm{x}$-axis and the value of accuracy in percent on the y-axis. 
Figure 14. Accuracy report

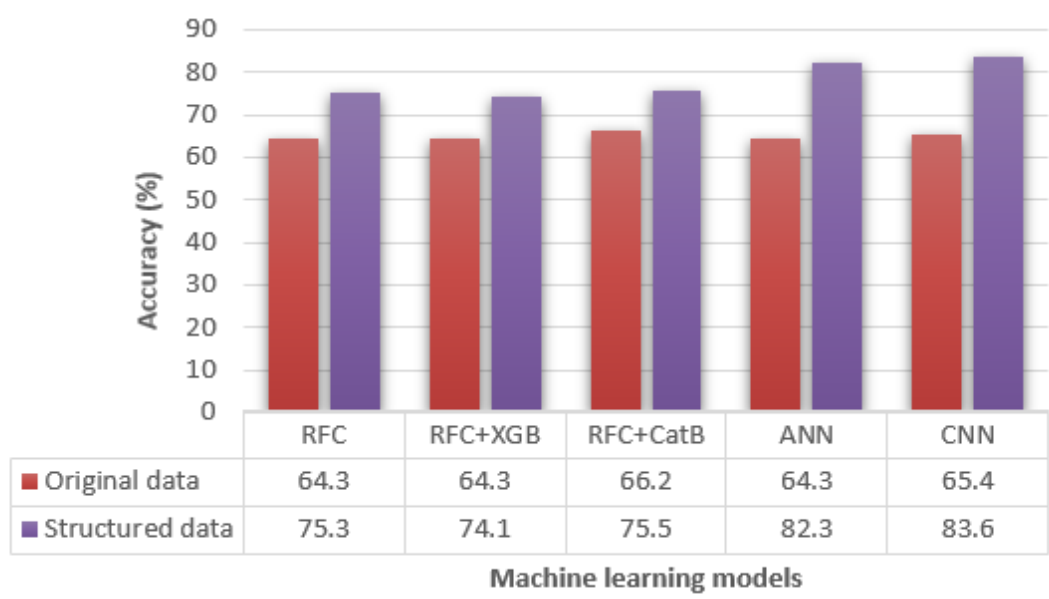

According to Huilgol, P.,2019, accuracy should be used where the distribution of classes is equal while F1-score is a superior measure where classes are imbalanced. Since we exploited experiments on a balanced dataset, we focused on analyzing the results of accuracy metrics as dominant over other classification metrics. If we look at the bar graph of the accuracy report, it is evidently supporting our first research question. Data model processed on expanded feature provides better accuracy results than original data features. We observe that starting from the first classifier (Random forest), we have an increase in the performance among the classifiers. Convolutional neural network obtains the best accuracy of $83.6 \%$ on structured data among the ensemble techniques and with respect to all the models. It is noteworthy that XGBoost algorithm obtains the lowest accuracy of $74.1 \%$ in comparison to all five models.

According to Powers, D. M., 2011, Precision or Confidence indicates the quantity of Predicted Positive cases that are correctly Real Positives. Precision is calculated by dividing the number of true positive labels by the number of true positive and false positives (Koehrsen, $W ., 2018$ ). In Figure 15, the $\mathrm{x}$ and y-axis represent the model name 
and precision score, respectively. The dark bars depict the precision trend of the original dataset classification whereas light-colored bars depict the precision trend of the structured dataset.

The precision score for the original dataset shows a similar trend for all five classification models (0.70), however, it shows more efficient results while utilizing neural networks for structured data classification. Surprisingly, the algorithms based on decision trees recorded a significantly low precision rate, whereas the best results are recorded by the CNN classifier (0.81).

Figure 15. Precision report

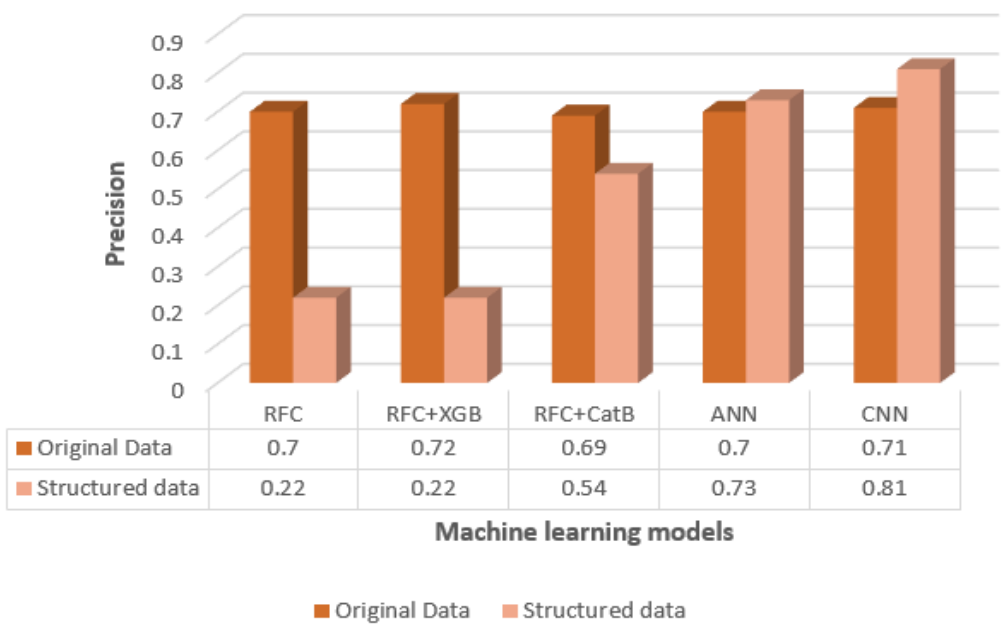

The next parameter of evaluation is Area under the ROC curve (AUC). AUC measures the entire two-dimensional area underneath the entire ROC curve which determines the quality of classifier (Narkhede, S., 2018). In Figure 16, the x-axis depicts the name of the classification model whereas the y-axis depicts the binary value of AUC. 
Figure 16. Area Under the curve report

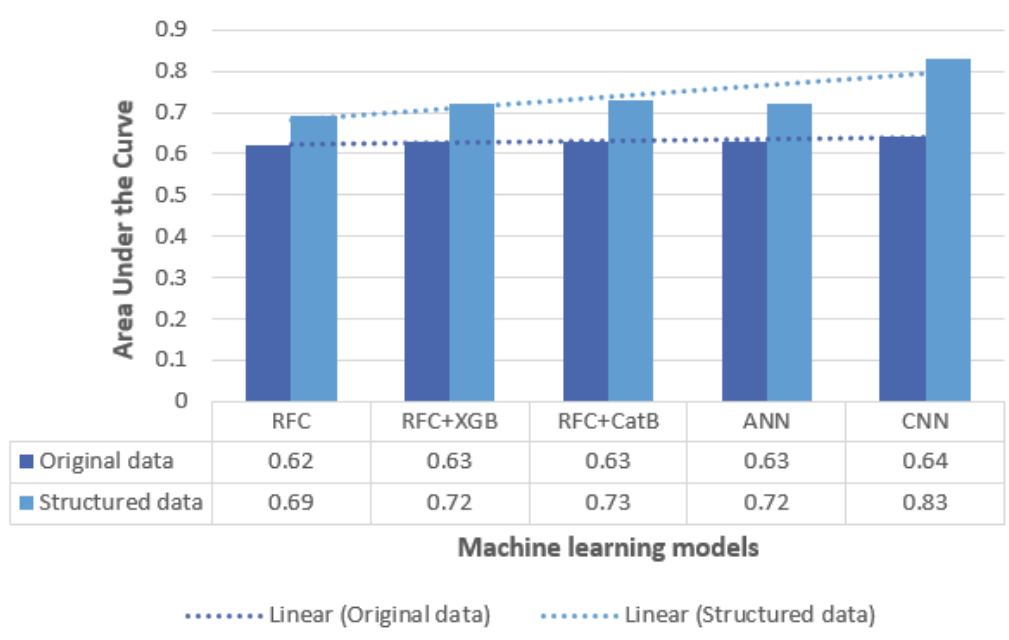

The bar graph reveals the same trend followed by each classifier when applied to original data set features, but considerable upturn can be seen via the trend line of AUC for the structured data set. The maximum area is curved under the CNN classifier (0.83), while AUC for the perfect classifier is equal to 1.

Powers, D. M., 2011 also stated that in Reverse to Precision, Recall or Sensitivity is the ratio of Real Positive cases that are correctly Predicted Positive. The recall is defined as the ratio of the number of true positive and number of true positives in addition to number false negative. It finds all the relevant cases for our evaluation report (Koehrsen, $W ., 2018)$.

In Figure 17, the classification models are represented on the $\mathrm{x}$-axis and the binary rate of sensitivity is depicted on the y-axis. The sensitivity score for original and structured datasets are symbolized using dark and light-colored bars, respectively. 
Figure 17. Sensitivity report

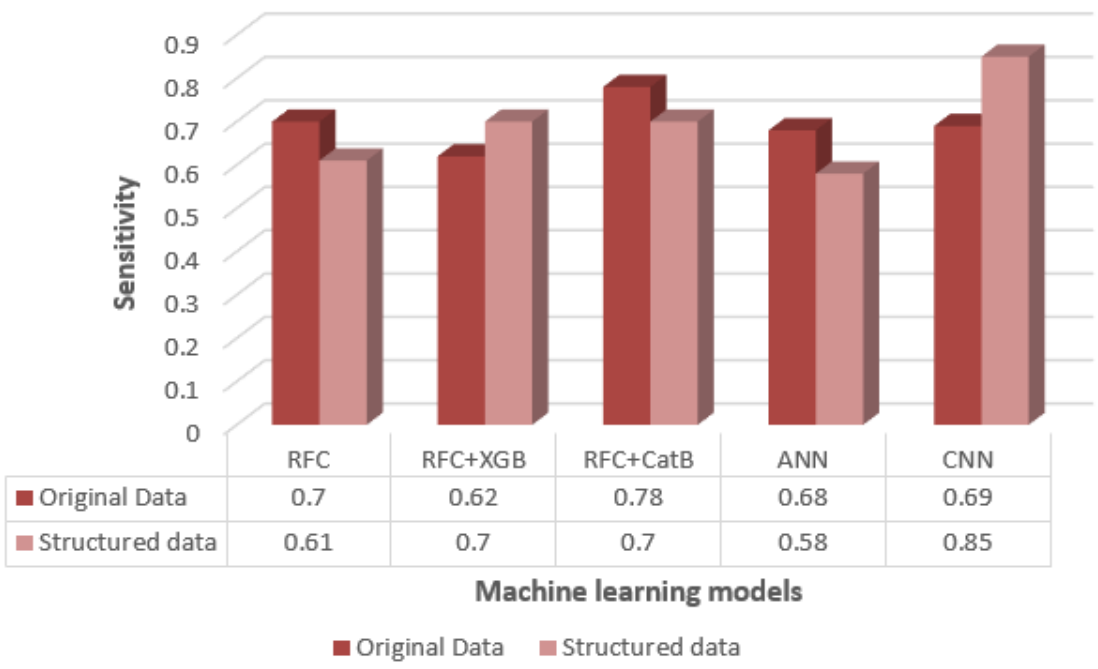

While assessing the sensitivity rate of all the models for both types of datasets, we can see that random forest classifier when applied to original data provides the worst recall value (0.61), whereas the best recall value is achieved by CNN classifier when applied on the structured dataset $(0.85)$. Another interesting pattern is shown by the two boosting algorithms (XGboost and CatBoost). The algorithms computed similar recall value on the structured data set $(0.70)$ but the trend of recall value on the original dataset is entirely different. The highest value of recall while evaluating the original dataset is processed by Catboost.

The next assessment factor of the model performance is the true negative rate (TNR) or Specificity. Mathematically, the specificity of a classifier can be calculated by dividing the number of true negatives to the sum of true negatives and false positives (Kumar, A., 2018). The rate of specificity is directly proportional to the number of true negatives and inversely proportional to the number of false-positive labels. Figure 18 
represents the specificity report of ensemble experiments with the algorithm names on the $\mathrm{x}$-axis and binary values on the $\mathrm{y}$-axis.

Figure 18. Specificity report

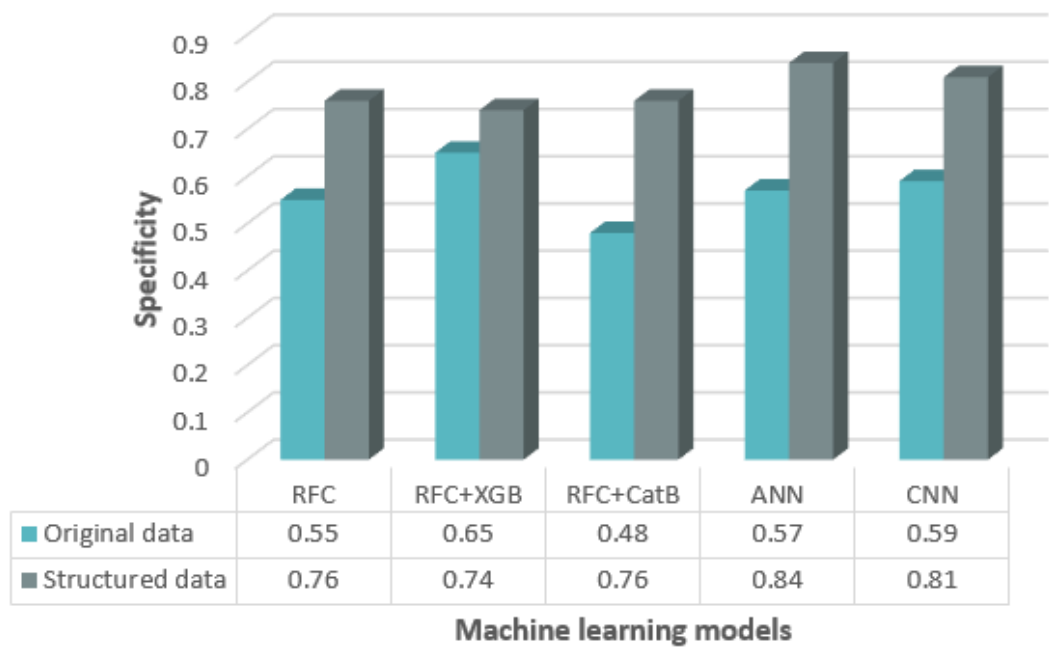

The specificity score determines the proportion of actual negative cases that are predicted correctly by the classifier. As seen in Figure 18, the specificity score is always superior for structured data labels than the original data labels. The highest specificity score is achieved by the ANN classifier (0.84), followed by CNN (0.81). Certainly, the Neural network outperforms the other classifiers.

The next classification metric is False Positive rate (Fall-out) which is termed Type 1 error which describes the number of classification labels that are incorrectly classified. The sum of FPR and TNR will always be 1 (PICO - What is False Positive rate? 2020). FPR can be calculated by dividing the total number of False-positive cases with a total number of negative cases $(\mathrm{FP}+\mathrm{TN})$. For a superlative classification model, False-negative score should be minimum because incorrect prediction can lead to significant monetary 
shortfalls (Shostak, T., 2019). In our case, a higher FPR rate means there are substantial chances that the model will ignore the probability listing of real online shoppers.

The following Figure 19 depicts the classifier name on the x-axis and FPR values on the y-axis. The blue-colored bars in the graph represent the original data classification FPR score and the green ones represent the structured data division.

Figure 19. False Positive rate report

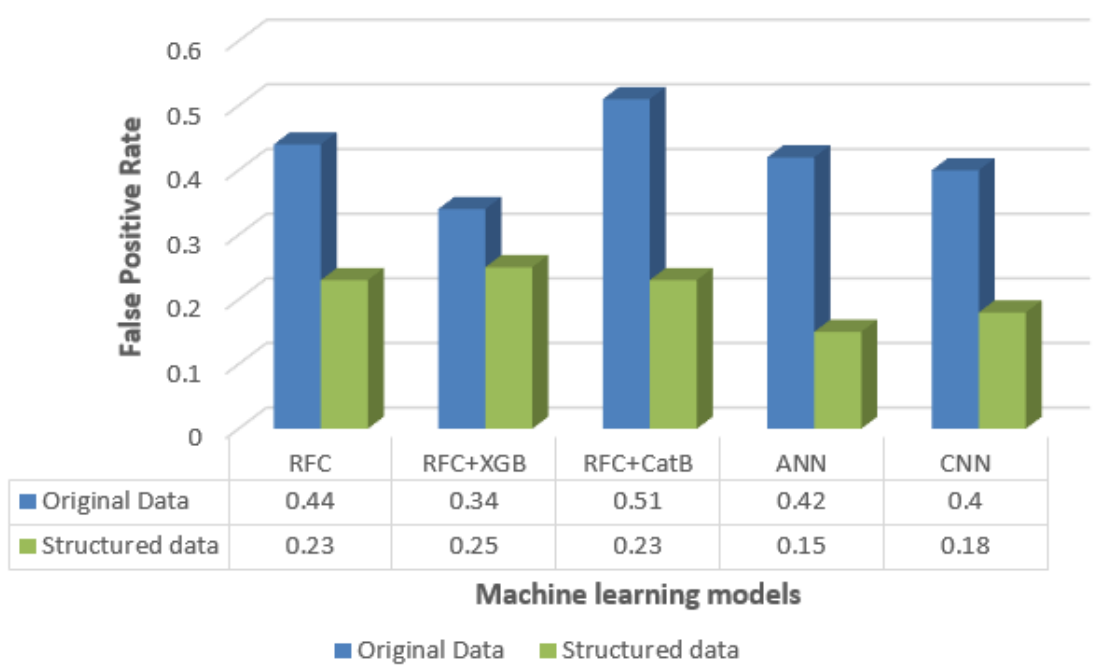

The figure above explains that feature expansion decreases the false positive rate by compelling numbers. The lowest FPR is hit by the ANN classifier when applied on structured data (0.15), followed by CNN classifier (0.18). The worst FPR is calculated by CatBoost classifier with original data features, producing 0.51 False positive labels in binary rate, which means 5 out of 10 times the CatBoost model produces wrong classification results. Hence, we can conclude that the structured data model with a neural network classifier is the best fit to obtain optimum results.

Equivalent to False positive rate, there is another matric that plays the same role in deciding the validity of the model. The false-negative rate is the miss out ratio of the 
classification and is termed as a Type 2 error (False Positives and False Negatives, 2020). The sum of TPR and FNR will always be 1 (False Positives and False Negatives, 2020). FNR is calculated by dividing the false-negative labels by the total number of positive cases (FN+TP).

Figure 20 shows the FNR report of our distinct machine learning experiments. The $\mathrm{x}$-axis and $\mathrm{y}$-axis represent the experiment name and FNR score, respectively. The orange bars in the graph represent the original data classification FNR score and the blue colored ones represent the structured data evaluation.

Figure 20. False Negative rate report

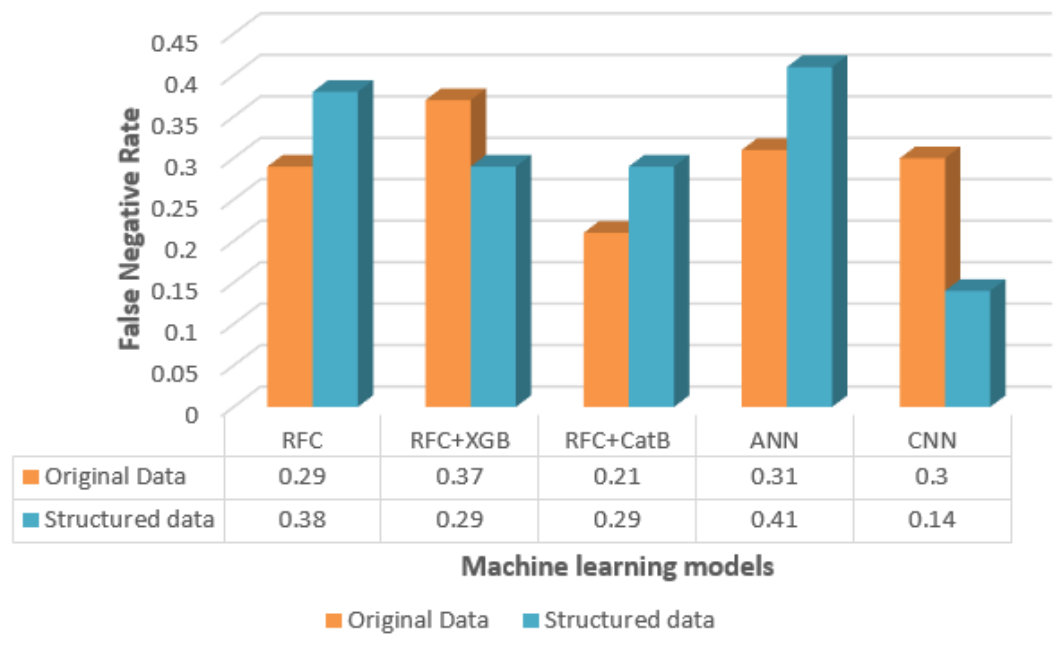

The figure directly above plots the FNR score for each model when applied to raw data and expanded data distinctly. Like FPR nature, the FNR score is inversely proportional to the quality of the model (Tyre, A. J. et al., 2003). The highest FNR score is computed by ANN classifier on structured data (0.41) whereas both boosting algorithms perform identical (0.29). It is notable that $\mathrm{CNN}$ when employed on structured data, the model significantly reduced the false-negative rate (0.14) which supports our earlier statement that structured data produces optimal results as compared to raw data features. 
To conclude, for our proposed model solution we aim to exploit expanded features as it delivers more reliable classification results for prediction tasks and overall attain exceptional evaluation scores over the existing dataset while utilizing neural networks. 


\section{Chapter 6: Design and Experimental setup of proposed model solutions}

In this chapter, we introduce our proposed model solutions. Our design solution is segregated in three distinctive portions: Purchase probability prediction using Instacart's (Instacart Online Grocery Shopping Dataset, 2017) historical data, propensity assessment using twitter (Twitter website, 2020) data, and purchase intent detection by employing Amazon (Amazon Fresh website, 2020) reviews. Finally, we integrate these three model solutions to suggest a use case framework that blends statistical and market empirical data with the goal of improving the grocery/retail sector.

\subsection{Model 1: Purchase probability prediction using historical data}

In this section, we propose a solution model by exploiting the historical data of Instacart. First, we review the system design of our predictive model and explain how the combined decision of the multiple models yields the improved performance of our solution. Next, we discuss the technical architecture of the algorithms used to design the proposed solution along with the algorithmic design details explained through pseudo-codes. Finally, we introduce the discussion on the chosen model designs.

System model: The structural design of our proposed solution built on historical data, is based on ensemble modeling (Caruana, $R$. et al., 2006) which has been lately in attention due to its abilities to provide improved outcomes by boosting the performance of each sub-model (Lessmann, S. et al., 2019). Specifically, in ensemble learning a set of prediction models termed as experts yield the final prediction of the problem domain (Singh, N., \& Rao, S., 2014). A single machine learning algorithm tries to study one 
premise and trains the data on it, whereas ensemble modeling learns via various hypothesis and merge them all to generate the optimum prediction.

The high-level architecture of our novel historical data evaluation model could be separated into three parts. First is the data processing part, which guarantees the expanded features are effective. We investigate the data and perform an imbalanced class setting before fitting it into the classifier. Second, which is the main contribution of our work is to build the prediction models to target the repurchasing of the products. And the last part is to route the crux by applying the ensemble technique to generate the final prediction.

\subsubsection{Design discussion}

In this section, we provide a supportive reason for choosing the design of our first model. Our framework is based on the ensemble learning concept (Caruana, R. et al., 2006). According to Verbeke, W. et al., 2012, ensemble learning use multiple independent base models implemented together which contribute to the voting process to yield better predictive performance as compared to baseline models.

By evaluating the literature review of ensemble models frameworks (Kumar, A. et al., 2019; Caruana, R. et al., 2006; Verbeke, W. et al., 2012), we integrate decision trees with deep learning techniques. The effective $\mathrm{CNN}$ algorithm is implemented along with random forest and XGBoost as a library of constructing models. The CNN framework is capable of extracting abstract and dominant features (Kiranyaz, S. et al., 2019), whereas decision trees help in quantifying the possible outcomes (Chen, T., \& Guestrin, C., 2016). While following Caruana, $R$. et al., 2006, we apply a hard-voting classifier that helps in selecting the best candidate for prediction aggregation. By exploiting statistical 
information, this framework serves the objective of finding economical strategies proficiently.

\subsubsection{Technical design of Segment 1}

We currently have raw data split into five relational databases. All the tables have a large number of features in the raw data (Instacart Online Grocery Shopping Dataset, 2017). If we involve all the features into our consideration, it will not only drastically impact the computational complexity but will also impact the performance of the model. So, we leverage the data by executing effective feature expansion as explained in section 5.2. The newly generated imbalanced data is then handled by applying synthetic minority oversampling techniques to avoid the risk of data overfitting. The target classes used for classifying the repurchasing trend are equally distributed by randomly replicating the minority class. The data is then split into two parts for training and testing using sklearn.model_selection module (Training and testing data splitting module from Scikitlearn library, 2007) at the beginning of our suggested model solution. Within the function, the test size $=0.2$ shows the percentage of the data to be used for testing purposes. 
Figure 21. Detailed technical design diagram (Model 1)

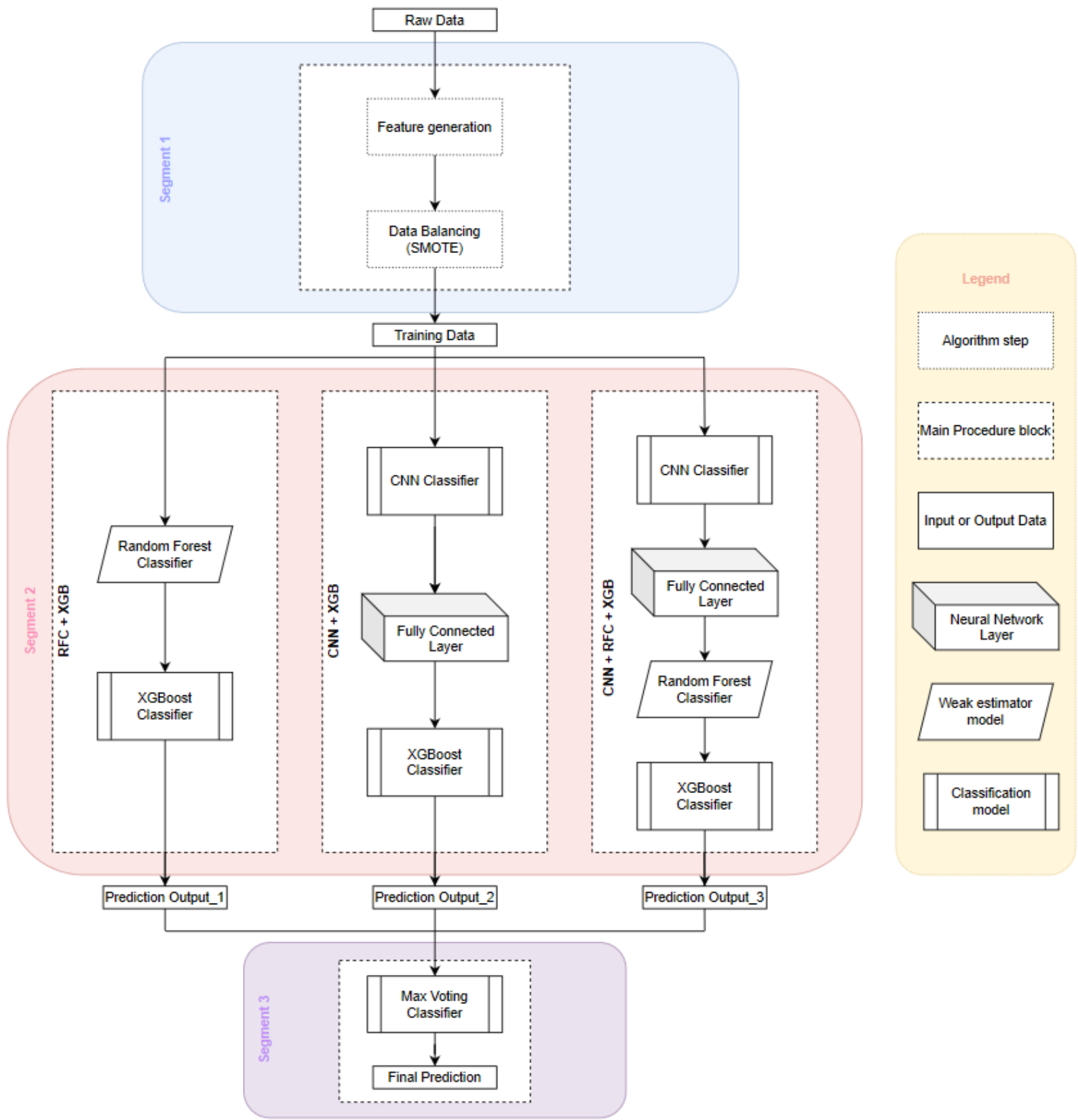

\subsubsection{Technical design of Segment 2}

This part is the elaboration of the detailed technical design based on Figure 21. In this part, we split the content by technical segments of our model design. Each procedural block in segment 2 consists of classification model algorithms. The details of each model 
used in the proposed solution are elaborated in Chapter 5. The content of this part of the chapter will focus on illustrating the data workflow.

From the experimental data dictionary, we utilized the raw data features to generate a set of extended new features. Then we feed the data with new features into a data balancing algorithm named SMOTE. After we process our training data, we split it into parts to feed the data into the procedural blocks of segment 2 to get the probability prediction results.

The novelty of our proposed solution is that we not only exploit the ensemble method but also generated new features that provide better prediction results as compared to raw data.

\section{a) XGBoosting using Base estimator RFC}

The first procedural block of segment 2 represents the eXtreme Gradient boosting algorithm that turns a weak base learner into a strong learner (Chen, T., \& Guestrin, C., 2016). We take random forest classifiers as a weak learner which increases the performance and helps in structuring a strong learner model (Breiman, L., 2001). By implementing the ensemble technique, we aim to reduce the loss function which determines the prediction errors.

Figure 22. Detailed Technical Design of Procedural Block 1

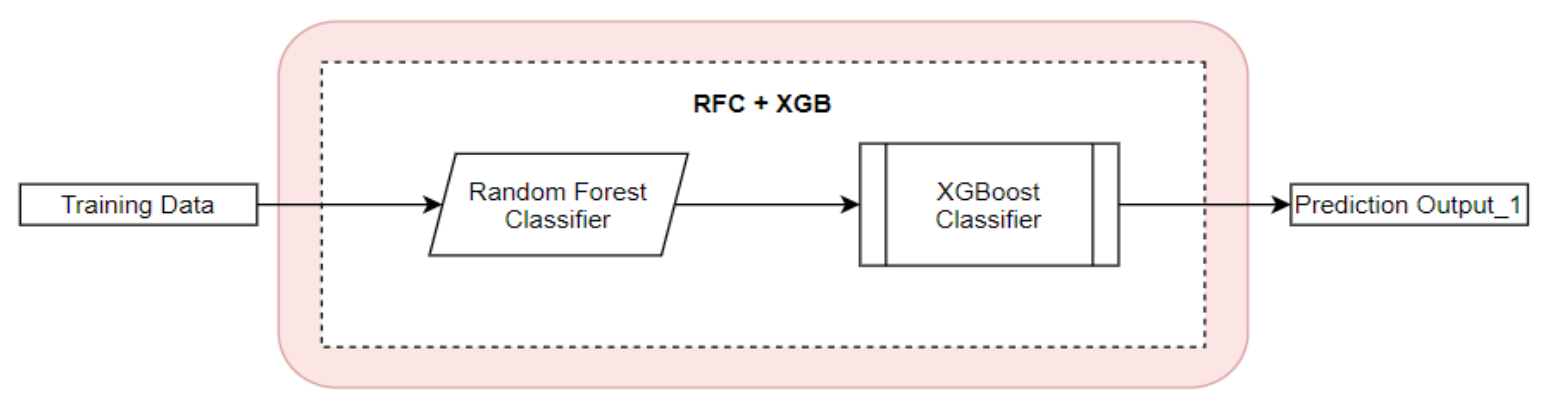


First, we take the base model random forest and fit the training data into it. Another learner XGboost is deployed on top of the existing base model to cover the loss function of the base learner. The resultant output is then stored as the first prediction (in Figure 22, Prediction output_1).

\section{b) XGBoosting using Base estimator CNN}

The second procedural block of segment 2 characterizes the same ensemble modeling structure as described in the section above, but with a different set of models. We use CNN as a base classifier for this part of the experiment.

Figure 23. Technical Design of Procedural Block 2

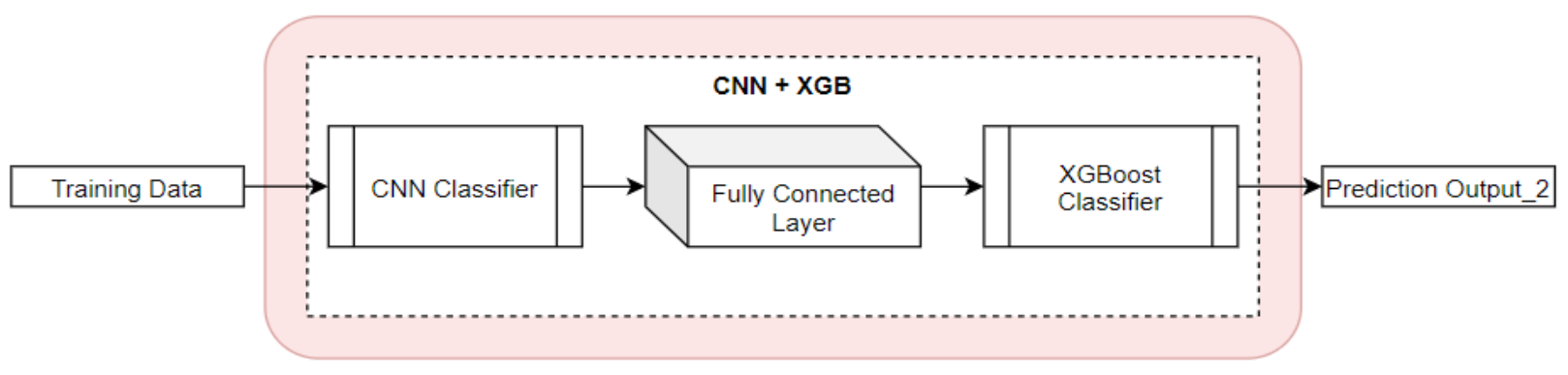

After processing the training data with $\mathrm{x}$ features in segment 1 , the next step is to reduce these features into a feature matrix scale of y features with the help of MaxMinScaler (MaxMinScaler, 2020). The processed feature matrix is then fed as input to the CNN layer.

The CNN layer is comprised of one or more neural units (Albawi, S. et al., 2017). Each neural unit performs classification and prediction based on the input data. The 1DCNN structure is formed of multiple layers: An input layer, in our case, input dimension is set to $(8,1)$; 1D-CNN layer with 32 kernels and ReLU activation; a dropout layer with a dropout probability $=0.1$; and a linear layer with a sigmoid activation function. 
Before calculating the final output to indicate the repurchasing prediction of the products, the dataset trained by the CNN classifier is extracted from the fully connected linear layer and is fed to the XGBoost classifier. This classifier tries to lower the loss function estimated by the CNN classifier by reducing the prediction errors. The output calculated by the XGboost model is then stored as the second prediction (in Figure 23, Prediction output_2).

\section{c) XGBoosting using Base estimator CNN + RFC}

In this subsection, we will describe the third procedural block of segment 3. This block comprises of design which is inherited from procedural blocks 1 and 2. We use CNN as a base model classifier and combine the trained data with an ensemble infusion of Random forest and XGBoost classifier.

Figure 24. Technical Design of Procedural Block 3

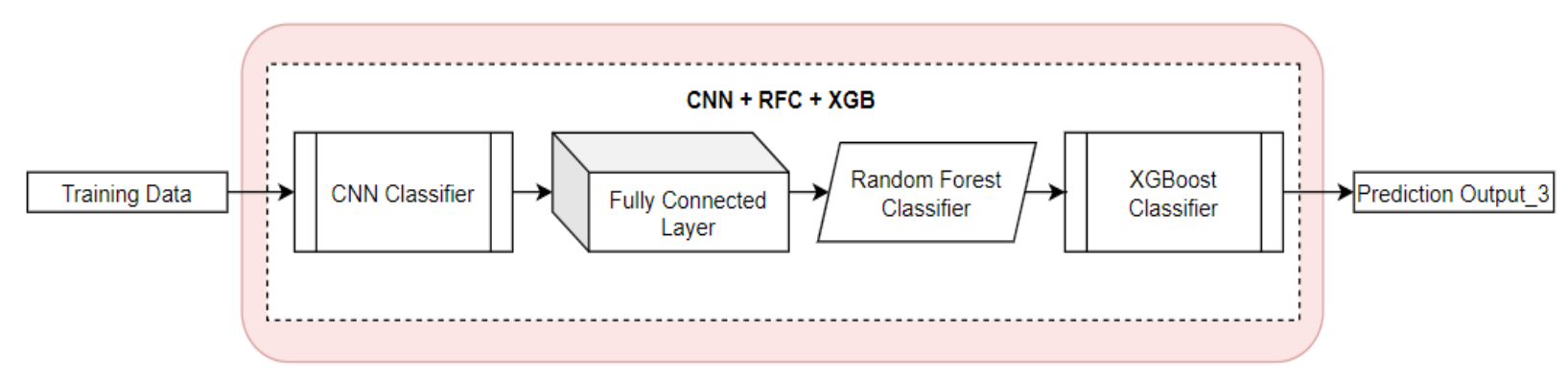

Like procedural block 2, pre-processed data from segment 1 is reduced to a feature matrix to obtain dimensional data. The processed feature matrix input is fed to the first layer of the $\mathrm{CNN}$ classifier. The trained data is then extracted from a fully connected dense layer of the neural network and fed to the random forest model for classification. The random forest classification model will help to reduce the classification errors and gaps of the CNN model (Yazdizadeh, A. et al., 2019). The fully trained random forest model is then 
provided as a base model estimator to the XGBoost classifier. This triple layer of classification models will help in boosting the prediction accuracy by lowering the loss function to the minimum (Yazdizadeh, A. et al., 2019). The final output provided by the XGBoost classifier is stored as the third prediction (in Figure 24, Prediction output_3).

\subsubsection{Technical design of Segment 3}

This section will describe the third and last segment of our historical data evaluation solution which will generate the final output. For each data point, three predictions have been made by each procedural block. Since we are dealing with calculating the probabilities for the classification problem, we use the maximum voting ensemble method to combine all three model predictions. The repurchase probability computed for each product in this segment is then used as input for our next solution models.

Let us denote our three classification models as $\mathrm{C}_{1}, \mathrm{C}_{2}$, and $\mathrm{C}_{3}$ that computes classification results for each input point $i$. The maximum voting classifier will determine the result for $i$ based on classifiers with the maximum number of classifications (Howal, S., 2017).

$$
V(i)=\operatorname{Mo}\left\{C_{1}(i), C_{2}(i), C_{3}(i)\right\}
$$

Where Mo denoted mode of classification results and $\mathrm{V}$ is the final classification result for input $i$.

Hence, each model prediction outputs are assessed proportionally according to their importance towards the forecast. The code to implement this technique is adapted from "A Comprehensive Guide to Ensemble Learning (with Python codes)" on analyticsvidhya.com (Singh, A., 2018) and from "Ensemble Learning in Python" on datacamp.com (Paul, S., 2018). 


\subsection{Model 2: Consumer propensity assessment using Twitter data}

In this section, we will investigate the public inclination towards topmost-selling products of Instacart. This methodology is proposed to study the relationship between social media data and the sales trend in the retail industry. We suggest a propensity assessment model that focuses on predicting short-term product preference based on userspecific real-time data. This approach forecasts the purchase preferences of the customers based on frequently changing social media data that shed light on their opinions, concerns, and feeling about highest re-purchased Instacart products extracted from model 1.

First, we describe the system of the model to understand our research approach by introducing a technical design diagram. After that, we discuss the twitter data retrieval procedure which will then be fed to our sentiment analyzer to categorize the preprocessed tweets into sentiment categories. Next, we discuss our propensity predicting algorithms with the help of pseudocode. At last, we present the design discussions of the algorithms.

System Model: To demonstrate this, this model is two-fold: The first frame analyses the sentiments of tweets being positive, negative, or neutral using NLTK's lexicon and rule-based sentiment analysis tool (VADER sentiment analyzer, 2020). The second model frame depicts the evaluation of user propensity towards certain products based on location using a machine learning algorithm. The focus of this model will be to derive a sales forecast for the products that are most popular amongst Instacart users. For this methodology, let us assume that the phenomenon of public interest on social media is directly correlated to the sales of a company. 
Figure 25. Detailed Technical Design diagram (Model 2)

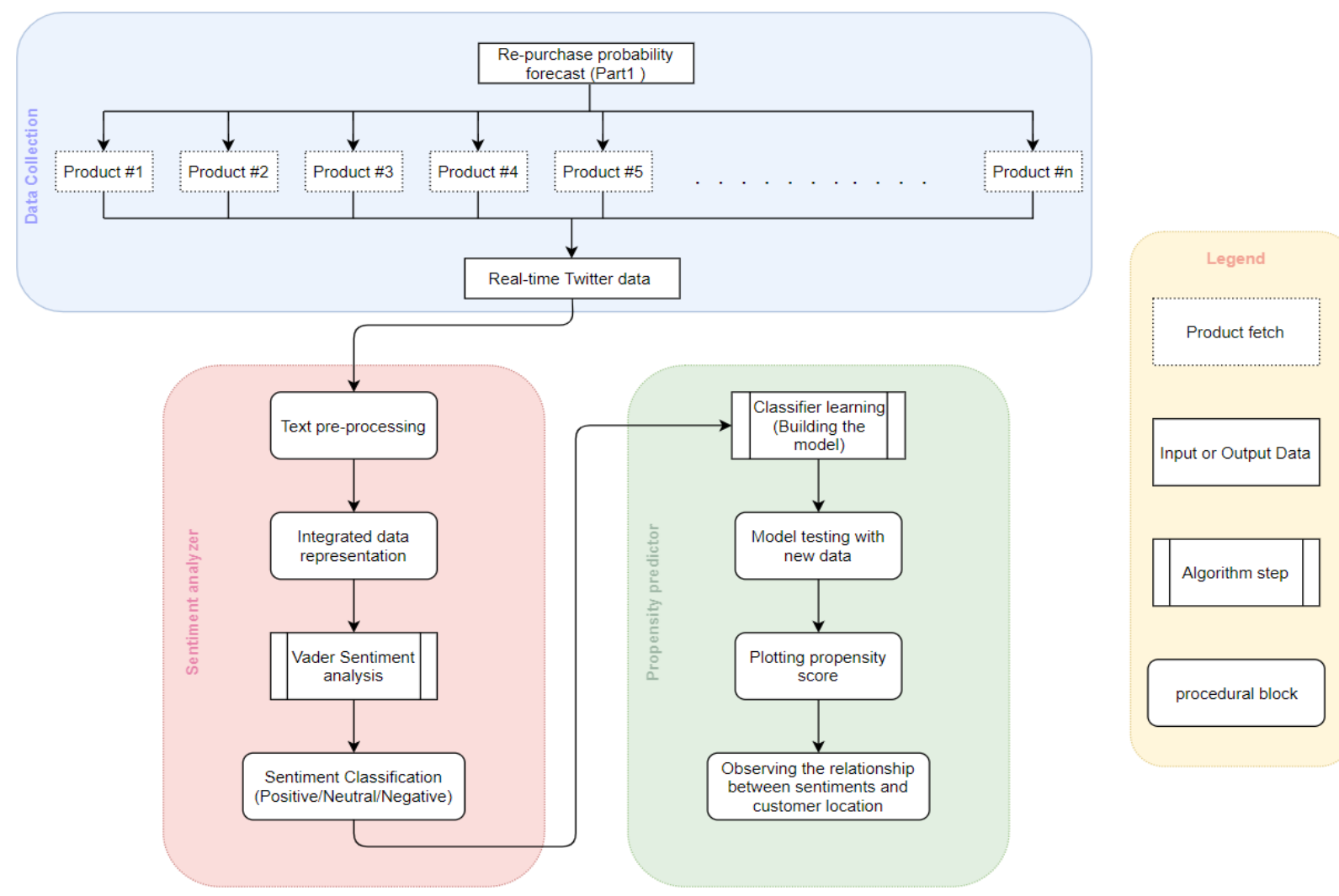

\subsubsection{Data Collection}

We focus our attention on the most re-purchased products of Instacart to understand the impact of social activities on product sales. We collected the tweets related to each product which highest-selling probabilities attained in model 1. The data is recorded over the week of mid-April 2020 using the Twitter application programming interface (API) (Makice, K., 2009). Twitter API is a programmed approach to data retrieval. It requires a unique set of authentication keys accessed through a twitter developer account.

We set the search query to each product's name, which then returns all tweets, retweets, and replies for the specified location and time condensed from the public data stream. We collected approximately 500 Tweets of the week associated with each query 
on the Twitter search API which delivers a purportedly random $1 \%$ of all publicly uploaded Tweets in Canada. However, the randomness in the quantity of tweets spawned a major challenge while assigning data to train, validation, and test sets. Please note that the data collected from twitter is unlabelled in terms of sentiment classifications.

\subsubsection{Design discussion}

Here we discuss the reason for choosing certain algorithms for this model task. We employed the linear support vector machine, which is claimed to be a state-of-the-art model for sentiment classifications (Phienthrakul, T. et al., 2009; Kumar, V.\& Subba, B., 2020; Han, K. X. et al., 2020). According to the author of Phienthrakul, T. et al., 2009, and Support Vector Machines from Scikit-learn library, 2007, SVM defines effective decision boundaries (hyperplanes) between categorical vectors by using kernel techniques. Hence, to categorize the text vectors generated from tweets, we apply SVM for optimized classification results.

While our other model choice is random forests, which is based on multiple decision tree concept. Each tree predicts the class value for every vector node and hence majority vote is considered as the final decision (Breiman, L., 2001; Liaw, A., \& Wiener, M., 2002). Such a robust ensemble technique is claimed to provide efficient results in the field of text classification (Hegde, Y., \& Padma, S. K., 2017). The comparison of metric scores of these two algorithms is elaborated in the next chapter.

\subsubsection{Sentiment analyzer}

The first frame of our propensity model comprises analyzing preprocessed tweets based on sentiment score of each tweet. Initially, due to the varying nature of lexicon used 
in microblogs, preprocessing techniques are implemented which extracts the pertinent content from the tweets while dropping out the irrelevant information. Extracting the quality data is considered as the most crucial step in text classification which directly influences the performance of the classifiers.

\section{a) Removing URLs}

To provide supplementary knowledge or to back the opinion presented in tweets, many users share URLs due to limited word restriction. However, crawling the link associated which each tweet is considered out of scope. Hence, we remove all the URLs with the help of defined functions which reduces the feature size.

\section{b) Removing special characters and punctuations}

In a compact blogging space, users may use any special characters, for example, “@” is used to mention other user handles, “\#” is used to specify the tweet category. Moreover, it is common to use punctuations for a clearer expression of emotions. In this step, we remove irrelevant characters and punctuations to avoid redundant features.

\section{c) Converting to lower case}

E-shouting is a common practice while posting opinions or describing emotions (Gokulakrishnan, B. et al., 2012). The capitalized words reveal the polarity of the tweets which could be useful for analysis (Gokulakrishnan, B. et al., 2012). Furthermore, users tend to write in an irregular casing (such as \#GrOceRyShoPPinG). Such word occurrences are handled using a regular expression operation library. 


\section{d) Removing stop words}

Common words such as like, the, be, are known as stopwords which do not ass any weight to classification process and hence can be neglected. In this step, we use NLTK (Natural Language Toolkit) (NLTK, 2020) in python which has a list of stopwords with language variations.

\section{e) Removing repetitions of letters, numbers, and spaces}

To express strong emotions, tweets often contain elongated words and spaces which are redundant from classification point. Hence, we disintegrate recurring characters in a word by leaving only two consecutive repetitions of the same character to identify the polarity. We also remove numbers and words that contain them using regular expression operation library.

The preprocessed tweets are used to measure sentiments. Initially, the tweets are an unlabelled set of data that includes processed text from user-generated tweets. We employ NLTK's state-of-the-art approach Valence Aware Dictionary and sEntiment Reasoner (VADER) (VADER sentiment analyzer, 2020) to prepare a training dataset for sentiment classification (Positive, Negative, or Neutral). The reason we chose VADER for sentiment analysis is that it is a rule-based sentiment analyzer constructed from a generalizable, valence-based, human-curated gold standard sentiment lexicon (Pandey, P., 2018).

For each given tweet $t$, we define a set of words $\left\{\mathrm{w}_{1}, \mathrm{w}_{2}, \mathrm{w}_{3} \ldots \mathrm{w}_{\mathrm{n}}\right\}$. For each $\mathrm{w}_{\mathrm{i}}$ polarity_score() method is used to obtain polarity indices which then are combined to 
compute the overall polarity of the $t_{i}$ tweet. Given a tweet $t$ with $n$-words and polarity scores $P_{i}$, we define compound score:

$$
\mathrm{C}_{\mathrm{t}}=\sum_{i=1}^{n}\left(P_{i}\right)
$$

The compound score defined by VADER analyzer contributes towards classifying the sentiment categories for each tweet $t$ :

$$
\text { If }\left\{\begin{array}{l}
C_{t}>=0.05, \text { Positive } \\
\left(C_{t}>-0.05\right) \text { and }(\mathrm{Ct}<0.05), \text { Neutral } \\
C_{t}<=-0.05, \text { Negative }
\end{array}\right.
$$

Where $\mathrm{C}_{\mathrm{t}}$ is the compound score of tweet $t$.

\subsubsection{Experimental design}

The final frame of our solution is to apply a classification model to predict the sentiment category for new tweets and to evaluate the propensity of users towards the products in the market. Here, we aim to create a supervised propensity assessment classifier based on the VADER analyzer (an unsupervised sentiment classifier). While the unsupervised approach is fine for the start, it will not yield clear results in domain-specific features using the open-source unsupervised method. To make the statistical algorithm work on text data, we first convert the text into numbers using the TF-IDF scheme (Term Frequency - Inverse Document Frequency). We use the TfidVectorizer class from python's Scikit-learn library (TfidfVectorizer Class from Scikit-learn library, 2007) to convert text into vectors. Now, before the applying our algorithm we split the data into training and testing set using sklearn.model_selection module (Training and testing data splitting module from Scikit-learn library, 2007), where the function test size $=0.2$ shows the percentage of the data to be used for testing purposes. To handle the imbalanced classes 
and to avoid the risk of data overfitting, we used the SMOTE technique (Chawla, N. V. et al., 2002). Finally, we employ Random Forest Classifier algorithm for prediction whose code is adapted from the "Random forest algorithm with Python and Scikit learn" article on stackabuse.com (Malik, U., 2020) "Random forest in python" on towardsdatascience.com (Koehrsen, W., 2017) and "Support Vector Machines" modules by Scikit-learn library (Support Vector Machines from Scikit-learn library, 2007).

After applying the prediction algorithm, we visualized the results to analyze the propensity of twitter users towards Instacart products based on user location feature. The visualization results will be addressed in the next chapter.

\subsection{Model 3: Purchase intention detection based on online reviews}

In this chapter division, we introduce our third model intending to comprehend customer's buying intent observing the most re-purchased products of Instacart retrieved from model 1 which predicts the products with the maximum probability of resale. In this model, we concentrate on predicting the purchase intent in the review retrieved from Amazon's website (Amazon Fresh website, 2020). Consumer purchase intention (PI) portrays the commitment by a user of buying or not buying any product provided by the retailer. We first begin by introducing the problem definition and system model design along with the technical design diagram. Following that, we explain the procedure followed to collect data from Amazon's website. Next, we cover the data cleaning and preprocessing step which is then fed to feature selection block. We then present the model implementation part by introducing the prediction algorithms used for classification analysis. Finally, we wrap the solution by discussing the chosen algorithm designs. 


\subsubsection{Problem definition}

Our approach is an extension of previous work by Atouati, S. et al., 2020. The author introduced a novel approach to identify negative purchase intent in a tweet. They also explicitly explained the disparity between sentiment and intent towards a specific product. While exploring the significant work done in the field of sentiment analysis (Aung, H. M. S., \& Pa, W. P., 2020; Chandra, Y., \& Jana, A., 2020), sentiment analyzer cannot be easily adapted to classify purchase intent present in the text. Moreover, positive sentiment (PS) does not imply positive purchase intent (PPI) and vice versa. Following table 19 depicts the difference between sentiment and intent present in a text which is a subset of our training data retrieved from Amazon Fresh's website:

Table 19. Difference between intent and sentiment

\begin{tabular}{|l|c|c|c|c|c|}
\hline \multicolumn{1}{|c|}{ Examples } & PPI & PS & NPI & NS & UPI \\
\hline $\begin{array}{l}\text { Moldy after 2 days of delivery. } \\
\text { Would not buy again. }\end{array}$ & & & $\checkmark$ & & \\
\hline $\begin{array}{l}\text { They arrived past their expiration } \\
\text { and had to be thrown out because } \\
\text { they were not edible }\end{array}$ & & $\checkmark$ & & $\checkmark$ & $\checkmark$ \\
\hline $\begin{array}{l}\text { Exactly what I needed for my } \\
\text { meal. Very fresh. }\end{array}$ & & & & & \\
\hline $\begin{array}{l}\text { A few bad or moldy raspberries } \\
\text { in the box. But rest I enjoyed } \\
\text { them immensely and will buy } \\
\text { them again! }\end{array}$ & $\checkmark$ & $\checkmark$ & $\checkmark$ & & \\
\hline $\begin{array}{l}\text { Received my limes at home in } \\
\text { Pandemic. I appreciate the } \\
\text { delivery, but I can't afford their } \\
\text { price. }\end{array}$ & & & & \\
\hline $\begin{array}{l}\text { Not happy with the strawberries I } \\
\text { received this time, but I do not } \\
\text { have time to go to the store. Will } \\
\text { probably end up buying them } \\
\text { again from Amazon. }\end{array}$ & $\checkmark$ & & & \\
\hline $\begin{array}{l}\text { Very fresh berries. I will order } \\
\text { them again this month before my } \\
\text { prime membership expires. } \\
\text { Might switch to Instacart then. }\end{array}$ & $\checkmark$ & $\checkmark$ & $\checkmark$ & & \\
\hline $\begin{array}{l}\text { Where PPI: Positive purchase intent, PS: Positive sentiment, NPI: Negative purchase intent, NS: Negative } \\
\text { sentiment. and UPI: Unknown purchase intent. }\end{array}$ & & & & \\
\hline
\end{tabular}


Atouati, S. et al., 2020 aimed to develop a binary classifier whether the tweet contain negative purchase intent context. Their main concentration was only on negative purchase intention. But we expanded the research scope by identifying all three categories of intent: Negative (NPI), Positive (PPI), and Unknown (UPI). We introduce three distinct binary classifiers for each intent class because purchase intent is expressed using relatively rich vocabulary which could confuse a multi-classifier, for example, the review "Very fresh berries. I will order them again this month before my prime membership expires. Might switch to Instacart then" contains positive sentiment, positive purchase intent for next order in the former half of the review. But the user expresses negative purchase intention in the latter half. Hence, to accurately evaluate such special cases and avoid classification noise, we introduce three binary classifiers for each purchase intent category.

\subsubsection{System Model}

The high-level structure of this model comprises of three distinctive fragments. We scraped user reviews posted for Instacart's most re-purchased products from Amazon's fresh website by specifying a characteristic user-agent string in the request header that allows servers to identify the source browser and operating system from which scraping request has been executed. Before feeding the data to automated proceedings, the manually labelling of each review is done to divide them under three categories of purchase intention (positive, negative, or unknown).

After that, under the data preparation block, we clean and prepare the collected reviews by categorizing and tagging part-of-speech for each review with the NLTK library in the Python programming language (NLTK.tag package, 2020). Next, we feed the preprocessed data to the feature selection block which calculates the pointwise mutual 
information for the tagged words which is then used to construct the neighborhoods by assessing word bigrams. Following that, we customize the cost function for our classifiers to be applied for the prediction. In the third fragment of the model, we employ our machine learning algorithms that exploit overlapping neighborhood construction of words and variant parameters of the cost function.

Figure 26. Detailed Technical Design diagram (Model 3)

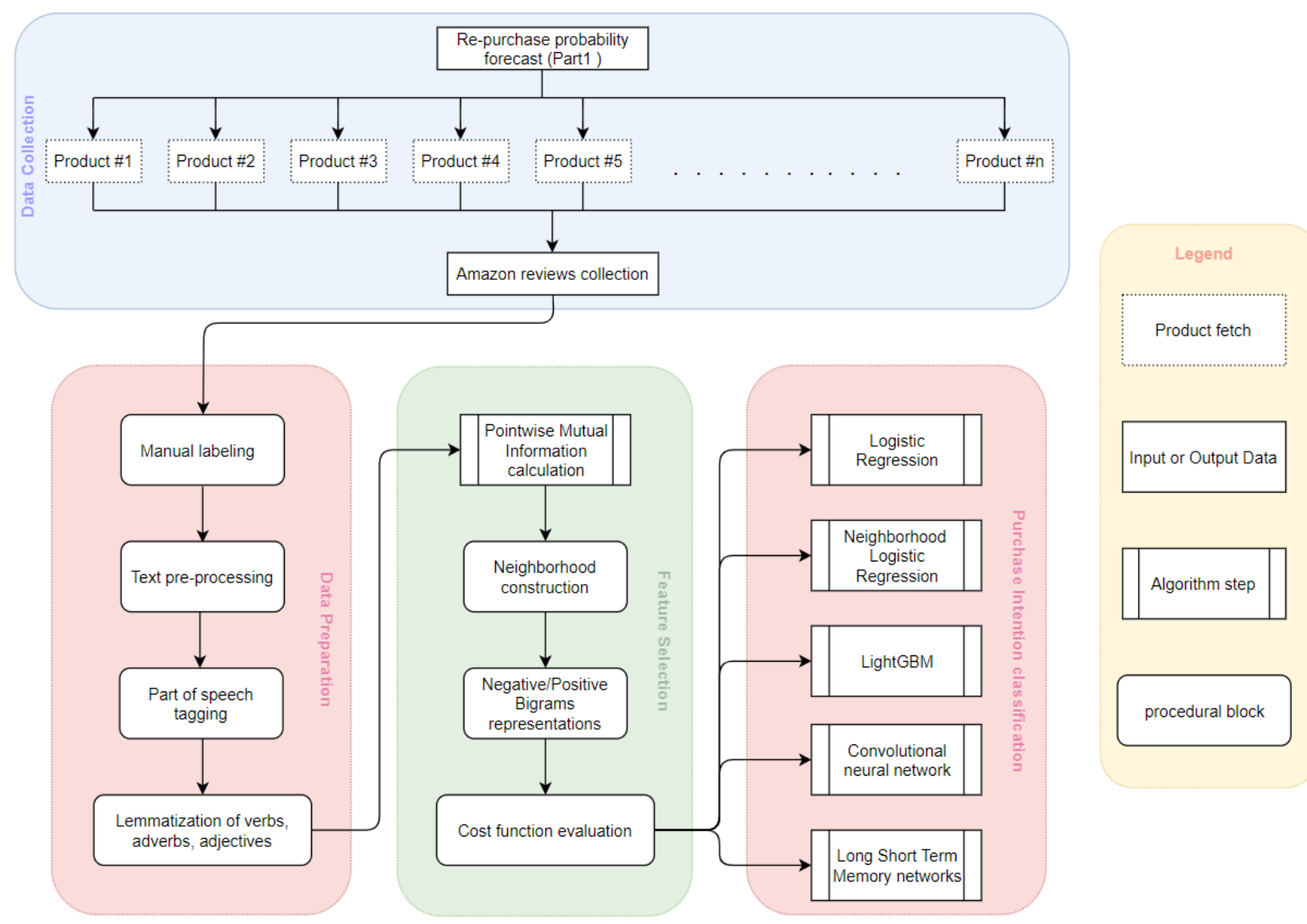

\subsubsection{Data collection}

In this section, we describe our data set used for this model. We concentrate on analyzing the products of Instacart with the highest re-selling probabilities, retrieved from model-1 results. For this study, we collected the online product reviews available through 
Amazon's fresh website (Amazon Fresh website, 2020) as of April 2020. We retrieved all the pages containing reviews for ten products utilizing the user-agent header string for source identification and the Beautiful Soup (Beautiful Soup, 2020) python library which is designed to scrape information from HTML and XML pages.

We set the URL search query to each product's customer review page. The Beautiful Soup function scrapes the class defined by the XML page which contains the text of reviews. We collected approximately 1700 user reviews associated with each product query. Each web page containing the customer review content was parsed to extract the class type "asize-base review-text review-text-content" from HTML page formatting. Since we are focused on identifying purchase intention commitment by the user, we only extracted user review text, neglecting other features such as Rating, Date of review, Helpful count. The quantity of data that we obtained bred one of the major challenges while splitting into training and testing sets. We split the data using sklearn.model_selection module (Training and testing data splitting module from Scikit-learn library, 2007), where the function test size $=0.2$ shows the percentage of the data to be used for testing purposes.

\subsubsection{Data preparation}

In this section, we introduce a detailed discussion on the preparation of web scraped raw text data for our classification model. Preparation of data is subdivided into four major steps:

\section{a) Manual labeling}

The labeling of purchase intention detection has not been studied yet, unlike text labeling packages for sentiment analysis (VADER sentiment analyzer, 
2020; Haque, $R$. et al., 2019). So, we manually labeled each review based on purchase intent availability in text. As mentioned above, each intent is binary classified under three different target labels: PPI, NPI, UPI. For example, in review "A few bad or moldy raspberries in the box. But rest I enjoyed them immensely and will buy them again!", the string of words that contains "will buy them again" represents positive purchase intent. Hence, the label PPI will be set to 1 and the other two labels would set 0 .

\section{b) Text cleaning}

To enhance the classification quality, we perform the stand preprocessing techniques explained in section 6.2.2. We remove URLs, special characters and numbers or words that include them from the text; convert the text into the lower case; we also remove extra spaces and alphabets replicating more than twice. The abbreviated words, such as "won't, shouldn' $t$ ", are extended to their full forms, such as "will not, should not". We do not remove stop words, as it might remove some useful sections of the text indicating purchase intent, such as negation words.

\section{c) Part-Of-Speech tagging (POS)}

POS tagging is a linguistic affiliated process that marks all the words in a text as their corresponding part of speech. For this step, we employ NLTK's POS tag module (Part-of-speech tag module from NLTK library, 2020) to identify the POS subcategory for each word, such as verbs, adverbs. 


\section{d) Lemmatization}

We execute word lemmatization by utilizing WordNet Lemmatizer (WordNet Lemmatizer, 2020) function from the NLTK python library. This function truncates the word to its root stem, such as "buying" will be converted to "buy".

\subsubsection{Feature selection}

Our research focused is to extend the previous work Atouati, S. et al., 2020, therefore we followed the same feature selection steps. Mainly, this section covers two computational segments: Pointwise Mutual Information and neighborhood construction.

To identify the association between a word in the review and its intent reflecting class, we use Pointwise mutual information formula (Statistical view of Pointwise mutual information, 2020). As mentioned by the author, the words with the highest PMI score have a high correlation with the intent class. For instance, there exist words $\left\{w_{1}, w_{2}, w_{3} \ldots w_{\mathrm{n}}\right\}$ for given review $r$. We set ppi associated with $r$ to 1 if $r$ indicates positive purchase intent, 0 otherwise. We evaluate the association between $w_{\mathrm{i}}$ and its class where ppir $_{r}=1$, statistically:

$$
\operatorname{PMI}\left(p p i_{r}=1, \mathrm{w}_{\mathrm{i}}\right)=\log \left(\frac{P\left(p p i_{r}=1, w_{i}\right)}{P\left(p p i_{r}=1\right) * P\left(w_{i}\right)}\right)
$$

Given class $p p i_{r}$, for $\mathrm{w}_{\mathrm{i}}$ mentioned in $r$, PMI decides if feature $\mathrm{w}_{\mathrm{i}}$ is of high correlation to the class. The high correlation is directly proportional to the significance of the feature for the learning algorithm.

The authors also found that since $\mathrm{P}\left(p p i_{r}=1\right)$ is constant, the right-hand side of the equation is increasing logarithmically which is directly proportional to the nominator. 
Hence, the correlation probability $\mathrm{P}\left(p p i_{r}=1, w_{\mathrm{i}}\right)$ serves adequately. In the case of rare words penalization, they exploited the Gaussian confidence interval at 95\% level. The adapted statistically representation for the lower bound confidence interval $(g)$ for rare word $w_{i}$ is:

$$
g\left(w_{\mathrm{i}}\right)=P_{e}\left(p p i_{r}=1 \mid w_{\mathrm{i}}\right)+z * \sqrt{\frac{P_{e}\left(p p i_{r}=1 \mid w i\right) * P_{e}\left(p p i_{r}=0 \mid w i\right)}{r_{w}}}
$$

Where $g\left(w_{\mathrm{i}}\right)$ is symmetric Gaussian interval, $P_{e}$ is conditional probability estimation, $r_{w}$ is the review where $w$ belongs to $r, z$ is a constant that represents the standard normal distribution value for a confidence level. Since we evaluate the Gaussian confidence interval at $95 \%$, in this case, $z=1.96$ as proved by Sullivan, Dr. L., 2020. It should be noted that the above equations are an adapted version of Atouati, S. et al., 2020. Similar equations are defined for negative purchase intent mentioned in a review $\left(n p i_{\mathrm{r}}\right)$ and rest are classified as unknown purchase intent $\left(u p i_{\mathrm{r}}\right)$.

For $n p i_{\mathrm{r}}$ :

$$
g\left(w_{\mathrm{i}}\right)=P_{e}\left(n p i_{r}=1 \mid w_{\mathrm{i}}\right)+z * \sqrt{\frac{P_{e}\left(n p i_{r}=1 \mid w i\right) * P_{e}\left(n p i_{r}=0 \mid w i\right)}{r_{w}}}
$$

For upi $i_{\mathrm{r}}$

$$
g\left(w_{\mathrm{i}}\right)=P_{e}\left(u p i_{r}=1 \mid w_{\mathrm{i}}\right)+z * \sqrt{\frac{P_{e}\left(u p i_{r}=1 \mid w i\right) * P_{e}\left(u p i_{r}=0 \mid w i\right)}{r_{w}}}
$$

Following, the author uses a python package Spacy (Honnibal, M., 2015) to extract the exact negation word dependency to directly distinguish the negative purchase intent. However, in extension, we extracted bigrams of affirmation dependency as well to extract 
word features like "definitely buying again", "fabulous quality" utilizing one-hotencoding. The rest features are categorized as unknown intent depicters.

The final feature selection process includes constructing neighborhoods for the expression that indicated purchase intent. As mentioned by the author in Atouati, S. et al., 2020 , in a review, the words representing substantial intentions are confined in relatively short frames. For example, in review "A few bad or moldy raspberries in the box. But rest I enjoyed them immensely and will buy them again!', the positive purchase intent expressed by the reviewer is represented in the latter half of the sentence. Hence, we follow the technique mentioned by the author to construct overlapping strings by splitting a review into multiple parts. These overlapping strings help in streaming potential words by eliminating the non-relevant words from the review.

For a review $r$ which contains words $\left\{\mathrm{w}_{1}, \mathrm{w}_{2}, \mathrm{w}_{3} \ldots \mathrm{w}_{\mathrm{n}}\right\}$, the author defines $h$ neighborhood of a word $w_{\mathrm{i}}$ represented in $2 h+1$ window, for every retained verb, adverbs, adjectives, and bigrams defined in above-mentioned feature selection steps. According to the matrix representation in Atouati, S. et al., 2020 for NPI, we formulated the similar equations for all three intent labels. For a given review $r$, feature matrix is represented as:

\begin{tabular}{|c|c|c|}
\hline $\begin{array}{c}\text { If } \boldsymbol{r} \text { represents positive purchase } \\
\text { intent }\end{array}$ & $\begin{array}{c}\text { If } \boldsymbol{r} \text { represents negative purchase } \\
\text { intent }\end{array}$ & $\begin{array}{c}\text { If } \boldsymbol{r} \text { represents neither positive nor } \\
\text { negative purchase intent (unknown) }\end{array}$ \\
\hline$p p i_{r} \in M^{h_{r} * f}$ with & $n p i_{r} \in M^{h_{r} * f}$ with & $u p i_{r} \in M^{h_{r} * f}$ with \\
$p p i_{r}^{i, h}=\left\{\begin{array}{c}1, \text { if } w_{i} \text { is } \text { in neighborhood } h \\
0, \text { otherwise }\end{array}\right.$ & $n p i_{r}^{i, h}=\left\{\begin{array}{c}1, \text { if } w_{i} \text { is in neighborhood } h \\
0, \text { otherwise }\end{array}\right.$ & $u p i_{r}^{i, h}=\left\{\begin{array}{c}1, \text { if } w_{i} \text { is in neighborhood } h \\
0, \text { otherwise }\end{array}\right.$ \\
\hline
\end{tabular}

Where $h_{r}$ represents the number of neighborhoods constructed for review $r, f$ represents the features and $w_{\mathrm{i}}$ is a word in $r$ which indicates the prominent intention of purchase. 


\subsubsection{Customizing Cost Function}

In this section, we follow a novel methodology introduced by Atouati, S. et al., 2020 to tailor the cost function of their employed algorithm. Formally, according to Krzyk, $K ., 2018$, the cost function is defined as an error function which provides a summation of prediction errors (loss function) for a training algorithm. Statistically, for every parameter $\theta$, the general equation of cost function $F(\theta)$ can be represented as:

$$
F(\theta)=\frac{1}{n} \sum_{i=1}^{n} C\left(h_{\theta}\left(x^{i}\right), y^{i}\right)
$$

Where, for iterations from 1 to $n, h_{\theta}\left(x^{i}\right)$ is defined as probability hypothesis function of logistic regression plugged in sigmoid and $y^{i}$ is a classification error function for $i^{\text {th }}$ training iteration. The hypothesis function manages to limit the cost function between 0 and 1. Atouati, S. et al., 2020 exploited the hypothesis function of logistic regression to interpret as a neural network. Generally, hypothesis function is represented as:

$$
h_{\theta}(x)=\sigma\left(\beta^{0} x\right)
$$

In our case, $x$ is our label $(x=p p i$ or $n p i$ or $u p i), h_{\theta}(x)$ conditional probability $P_{e}$.

For logistic regression, we expect hypothesis to evaluate between 0 and 1, hence sigmoid $(\sigma)$ is used:

$$
P_{e}=\sigma\left(\beta^{0} x\right)=1 /\left(1+e^{-\left(\beta^{0} x\right)}\right)
$$

Since our labels $x$ is 1 or 0 :

$$
C\left(P_{e}, x\right)=\left\{\begin{array}{c}
-\log \left(P_{e}\right) \text { if } x=1 \\
-\log \left(1-P_{e}\right) \text { if } x=0
\end{array}\right.
$$




$$
C\left(P_{e}, x\right)=-x \log \left(P_{e}\right)-(1-x) \log \left(1-\left(P_{e}\right)\right)
$$

The author customized the hypothesis function (in our case, $P_{e}$ ) to imitate a neural network by injecting the neighborhood feature matrix constructed in the above section. Hence, by following their approach, our customization of the cost function can be represented as:

$$
P_{e}=\max _{1 \leq i \leq h_{r}} \sigma\left(\beta^{0} x_{r}^{i}\right)
$$

Where $h_{r}$ is the number of overlapping neighborhoods constructed on review $r$. In other words, the author in Atouati, S. et al., 2020 is computing the maximum value of the sigmoid function for every neighborhood.

\subsubsection{Customizing Loss Function - An Extension}

To extend the previous work Atouati, S. et al., 2020, of customizing the cost function for the logistic regression algorithm, we focus on tailoring the loss function for the LightGBM (Ke, G. et al., 2017) algorithm in this section.

Theoretically, the loss function is an error in predicting a data point correctly, whereas cost function is the sum of loss functions that is the summation of all loss function error in a training set (Dhiraj, K., 2019). As we concentrate on binary classification, the loss function for LightGBM (Ke, G. et al., 2017) is the same as used in logistic regression:

$$
C\left(P_{e}, x\right)=-x \log \left(P_{e}\right)-(1-x) \log \left(1-\left(P_{e}\right)\right)
$$

By following the idea of Dhiraj, K., 2019, we customize the loss function by such means that it minimizes the false-negative classifications. To conduct the same, we 
magnify right-hand side of the equation (12) with a higher value constant $(\alpha)$ than the label 0 and 1 , that means $\alpha>1$ :s

$$
C\left(P_{e}, x\right)=-\alpha * x \log \left(P_{e}\right)-(1-x) \log \left(1-\left(P_{e}\right)\right)
$$

Finally, we define a function for the LightGBM algorithm, that uses our customized loss function for both validation and training. To derive gradient descent and hessian, we evaluate calculus derivates of the equation above by setting $\alpha=2$.

Algorithm 1 Customizing gradient descent and hessian in the loss function (Grover, P. 2018)

1. def custom_loss_train (y_true, $\left.y \_p r e d i c t i o n\right)$

2. $\alpha=2$

3. res_error $=$ difference of y_true and y_predict at a data point

4. if res_error $<0$ then

5. gradient $=(-\alpha * 10.0 *$ res_error,$-\alpha *$ res_error $)$

6. $\quad$ hessian $=(\alpha * 10, \alpha)$

7. return gradient, hessian

8. end def

9.

10. def custom_loss_valid (y_true, $\mathrm{y} \_$prediction)

11. $\alpha=2$

12. res_error $=$ difference of y_true and y_predict at a data point

13. if res error $<0$ then

14. loss $=\left(\left(\right.\right.$ res_error $\left.\left.{ }^{\alpha}\right) * 10.0, \alpha^{\text {res_error }}\right)$

15. return "custom_loss_valid", mean(loss), boolean whether higher $\alpha$ is better.

16. end def

\subsubsection{Experimental design}

This section concentrates on the experimental setup for our extended approach to predict the purchase intentions of the Amazon reviewers. We compare our methodology by employing five different machine learning algorithms: 1) an LSTM-based model (Hochreiter, S., \& Schmidhuber, J., 1997); 2) CNN model (Albawi, S. et al., 2017); 3) Tailored LightGBM (Ke, G. et al., 2017); 4) Tailored NeighLoR (Atouati, S. et al., 2020); 
5) Binary logistic regression (Kleinbaum, D. G. et al., 2002). Each algorithm is exploited separately for binary classifying the three labels: Positive purchase intent (ppi), negative purchase intent (npi), and unknown purchase intent (upi).

First, in the discussion, we train our LSTM model based on the Keras API provided by the Tensorflow library of python. In our case, LSTM consists of an input layer with dimension $=50$, an LSTM layer with 32 kernels, an activation layer set to ReLU, a dropout layer with probability 0.5 , and a sigmoid activation function before output layer. The performance of the model is calculated in terms of accuracy, F1 score, precision, and recall.

Next, we employ a convolutional neural network for three binary labels with classes 0 and 1 . We create a convolutional neural net based on the sequential stack method. The network mostly contains a standard linear dense layer which defines input, weights, and outputs. In our case, the input consists of sentences that are converted into a one-hot matrix and we define ReLU as the activation function. Next, we have two dense layers that consist of a dropout probability of 0.1 , which randomly removes data to confiscate overfitting. We assign sigmoid and softmax activation function to the two dense layers, respectively. At last, we compile the model to evaluate the performance.

The third evaluation is done using LightGBM. We define default LightGBM by injecting a customized cost function approach introduced in Atouati, S. et al., 2020, and employ our extended loss function for both training and validation while compiling the binary classifier. By default, the learning rate set to 0.1 and 100 number of iterations. We assign Mean Absolute Error (MAE) and Root mean squared error (RMSE) metrics and 10fold cross-validation over labeled data in terms of accuracy and F1score to measure model performance. 
The next model we utilized is NeighLor, which is introduced by Atouati, S. et al., 2020. The author exploited logistic regression by inducing neighborhood matrix with a 7word window. They ran gradient descent on customized cost function with a maximum of 5000 iterations and $10^{-4}$ tolerance rate. Besides, we inject out customized loss functions for training and validation sets. We evaluate the performance of the model using 10 -fold cross-validation based on accuracy, precision, recall, and F1 score.

At last, we apply simple logistic regression for binary classification of the labeled data. To map the binary classes ( 0 or 1$)$, we define a threshold of 0.5 , in other words, input data point producing output more than 0.5 is classified as class 1,0 otherwise as mentioned in Harrington, $P$., 2012. The model performance is assessed based on accuracy, precision, F1 score, and recall for consistency with other models.

The generic pseudo-code for our purchase intention classifier is as follows:

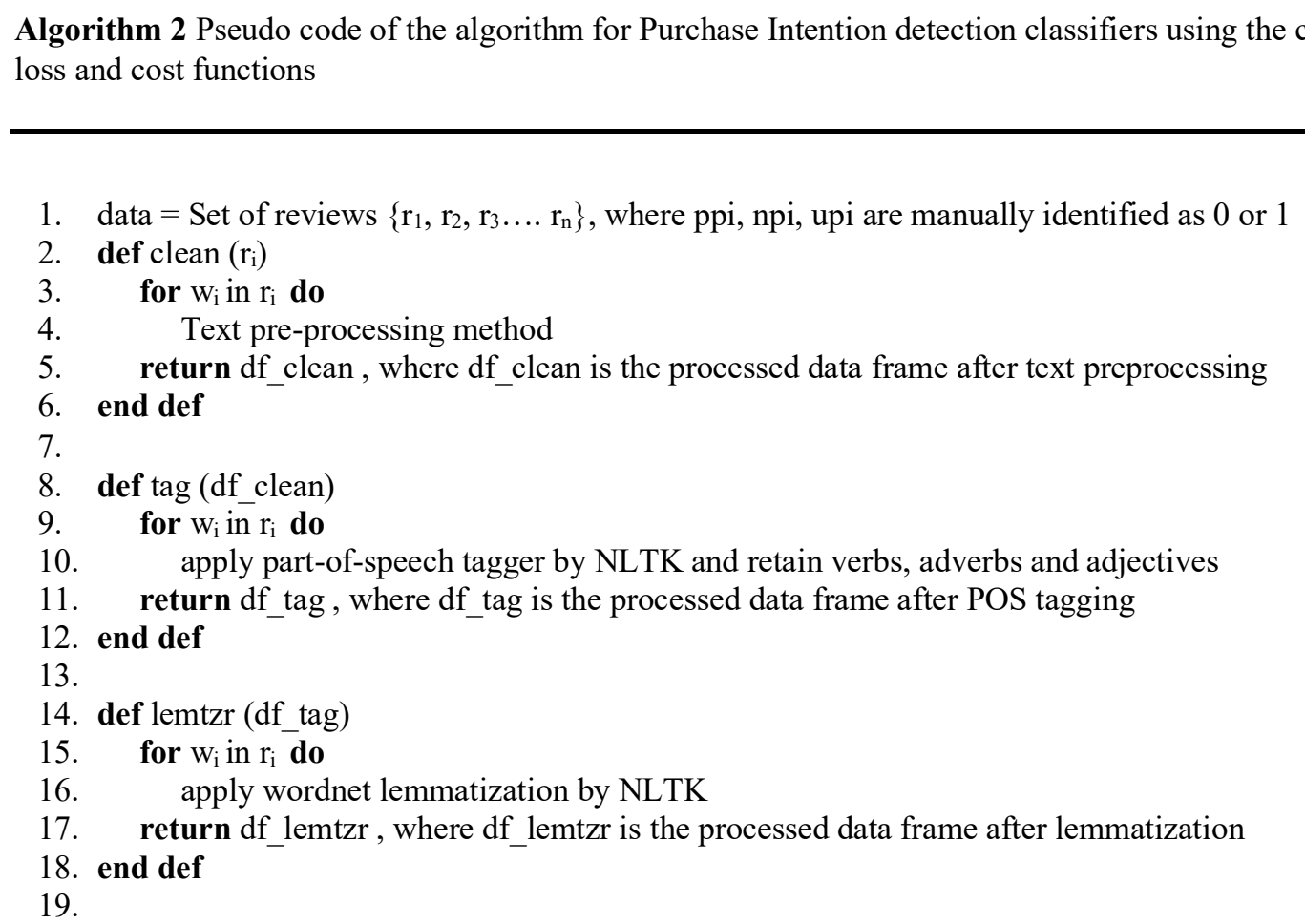




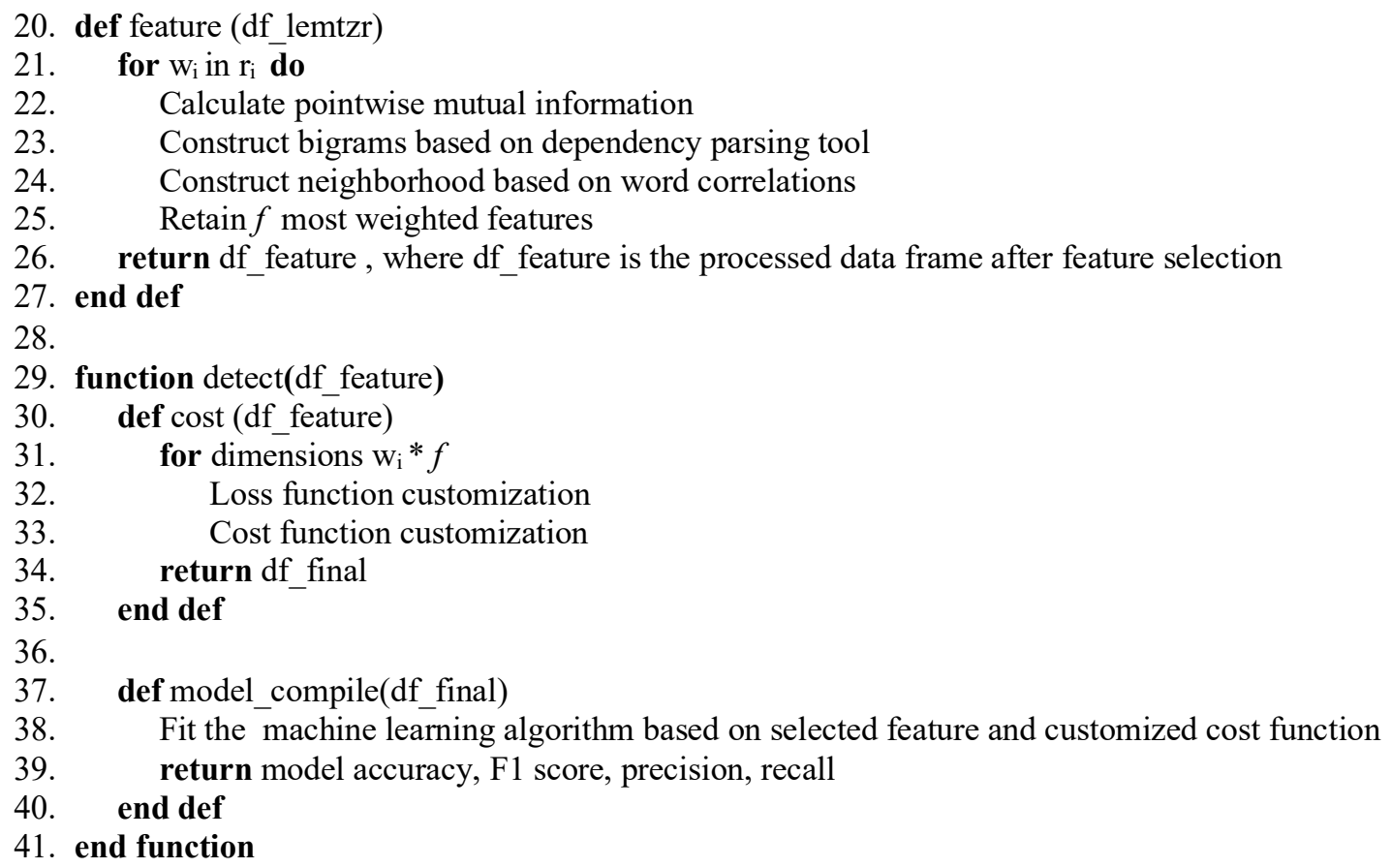

For applying the prediction model to analyze customer's purchase intention, we first disintegrate the words in a review $r$ using tokenizer function. For each word $\mathrm{w}_{\mathrm{i}}$, we apply text processing methods (mention in section 6.3.4) to eliminate noise from the dataset. Next, for the feature selection task, we first apply the POS tagger and retain words that are tagged as verbs, adverbs, and adjectives. The retained words are then lemmatized using word lemmatizer function. The lemmatized words are then used to find pointwise mutual information between words to construct the n-word bigrams by exploiting the dependency parsing tool. Following that, we construct overlapping neighborhood windows that might contain bigrams and adjectives. Finally, we feed the pre-processed data containing selected features to the customized machine learning algorithms for metrics evaluation. 


\subsubsection{Design discussion}

This section provides the rationale for electing specific algorithms for the task of predicting customer intensions. Since we followed Atouati, S. et al., 2020, we aim to choose the algorithm that is demonstrated to be coherent than logistic regression (the base algorithm used in Atouati, S. et al., 2020), thus we apply LightGBM as our primary algorithm. According to Ke, G. et al., 2017, LightGBM is built on a histogram approach that bins continuous elements together which results in efficient training speed and low memory usage. Such parallel learning support provides the capability to perform consistently on larger as well as reduced datasets. Therefore, this algorithm suits best to analyze the fluctuating quantitive nature of social media data.

As we mentioned before, model 3 provides an adequate extension to the work done in Atouati, S. et al., 2020. Hence, we opt to implement the author's proposed NeighLoR to demonstrate extended algorithmic advantages and improved functional results. Since NeighLoR is an adoption of the logistic regression algorithm, we also apply the latter to compute the result comparison between the two. By comprehending the model choices from related works (Sakar, C. O. et al., 2019; Ding, X. et al., 2015; Haque, R. et al., 2019), we also employ neural networks to execute a challenging assessment of our framework.

\subsection{Integrated model Impact}

In section, we present the integrated structure of our three model designs. Here, we briefly discuss the assembling of the integrated model to employ a mature use case . To recall, we start the design model by utilizing publicly available Instacart data to assemble model-1 which predicts the repurchase probability for each product revealed in the dataset. 
Then, we drive our next models based on products with maximum reselling probabilities. We built the propensity analyzer model, aiming to understand the inclination of users towards the products by employing sentiment analysis. This model evaluates the propensity for selected products based on the location of the user.

Finally, our main model-3 is based on previous work Atouati, S. et al., 2020 targeting to analyze the user purchase intention by evaluating Amazon Fresh reviews. We follow the approach mentioned by the author, which is to construct a neighborhood matrix for each intent category. Then we also employ the customized cost function proposed by the author while introducing a supplementary extension in the loss function.

The following figure represents the integration of the model solutions:

Figure 27. Integrated proposed solution

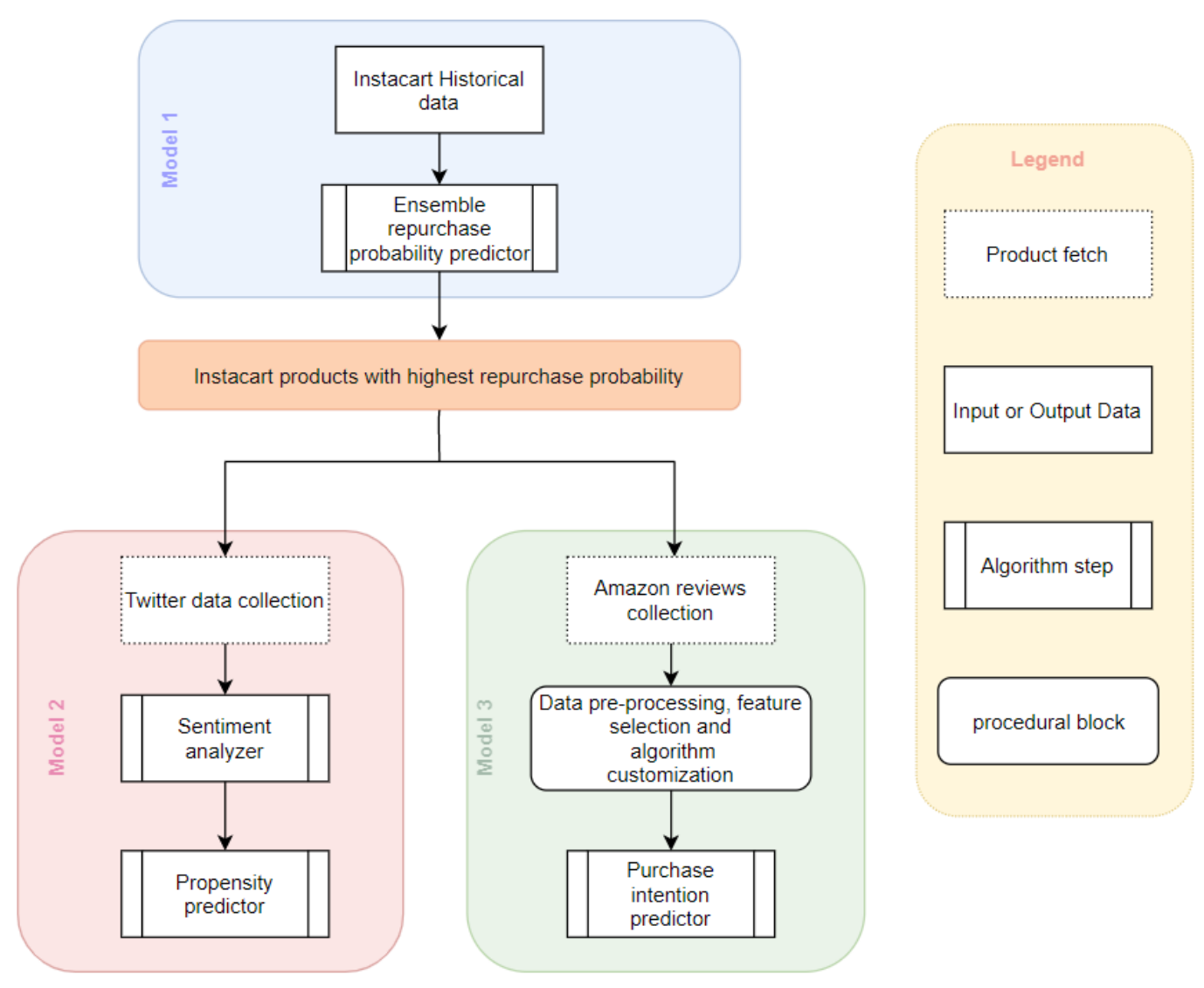


To point out the advantages of our three models, the integrated version combines the market mix models while learning from multiple machine learning algorithms. This incorporated structure of three models provides a platform to easily monitor individual model performance. However, it is capable to increase the overall execution time and deployment complexity.

\subsection{Potential real-life Use Case of the Proposed Solution}

In this section, we will intricate the use case application of our proposed solution that we designed in sections above. This section illuminates the entire reason for developing a novel machine learning model that predicts user purchase intention by assessing user propensity and probability of the products to be re-purchased. The main objective of this section is to imply how to implement the proposed solution as an application that shall benefit the retail market growth.

The aim of developing the use case is to implement a lite, easy-to-access application for the retailers or suppliers of the Instacart. The use case outlet is preferred as a graphical web application so that users can access the web page remotely with the help of its URL. We favor an all-python execution to sustain the consistency of the system components.

The overall architecture of the design is demonstrated in Figure 28. The application design consists of three prominent layers i.e. data storage layer, data preprocessing layer, graphical user interface layer. 
Figure 28. Use case Architecture

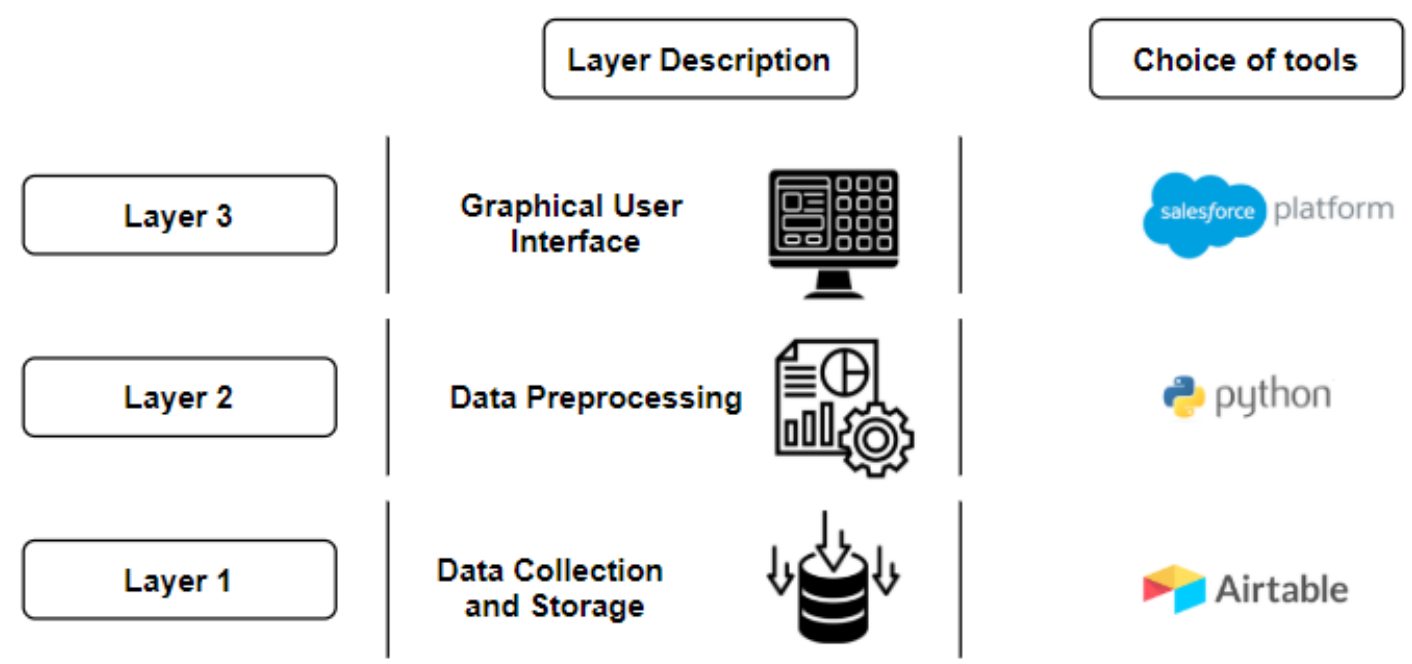

The base layer of building the desired web application is the data collection and storage layer. For the basic implementation, we use the code to collect the data and then store it to the database.

The second layer of the application is data processing. This layer serves as the spine for this use case. In this layer, we deploy all the proposed solution's modules from chapter 6.

The third and last layer is the front-end product i.e. graphical user interface for the user input and predicted output. Demo adaptation of the web page has a GUI for users to fill the department name, aisle name, and the product name with an output of the probability prediction result of the selected product.

With the help of the predicted results of the selected product, the retailers/suppliers can determine the user propensity and purchase intention towards a specific product. Hence, this could help the stakeholders to determine the kind of discount offers to be provided to the customers that would result in the highest increase in profits. It can support 
supermarkets to draw or retain consumers who may otherwise purchase food via another channel; this model design will generate increased competition for local grocery stores and the business as a whole. It would have substantially increased jobs in the grocery market by driving increased competition and rising employees' marginal wages.

\subsubsection{GUI Design discussion}

This section introduces the products used to assemble the application and the reasons to choose certain software tools.

For the first layer of data collection and storage, we choose Airtable (AirtableProduct, 2012) as it provides flexibility to store different types of data such as tabular data, audio files, images, pdfs. It offers an easy to use interface for accessing the database in the form of a GUI. This software provides suppleness to extend database features for the prospects of this project. For instance, if we plan to extend this research to construct a prediction model that analyses the customer traffic on the webpage of the retailer, we can get the training data straight from the same database without linking it to another source.

The back-end language that we choose for layer 2 is python. All our source codes implemented in the jupyter notebook are deployed in this layer.

In the third layer of our architecture, we choose the Salesforce lightning Platform (Salesforce Platform Lightning, 1999) to develop the graphical user interface of our application by exploiting the python libraries. In addition, we use another tool Salesforce platform integrated with Heroku (Heroku - Salesforce.com Inc, 1999) to deploy and run the developed interface like a web application.

To integrate Airtable with the Salesforce platform, Airtable provides a robust API to collaborate with other platforms (Airtable-Automate your work, 2012). Many 
automation tools such as Zapier (Zapier, 2011), Automate.io (Airtable Integrations, 2016) could be used to integrate Airtable with the Salesforce platform.

Figure 29 is the graphical user interface demo of our use case web application.

Figure 29. Web application GUI

\section{User's purchase intention identification - Dashboard}

- Please select the desired product by selecting the department and aisle name
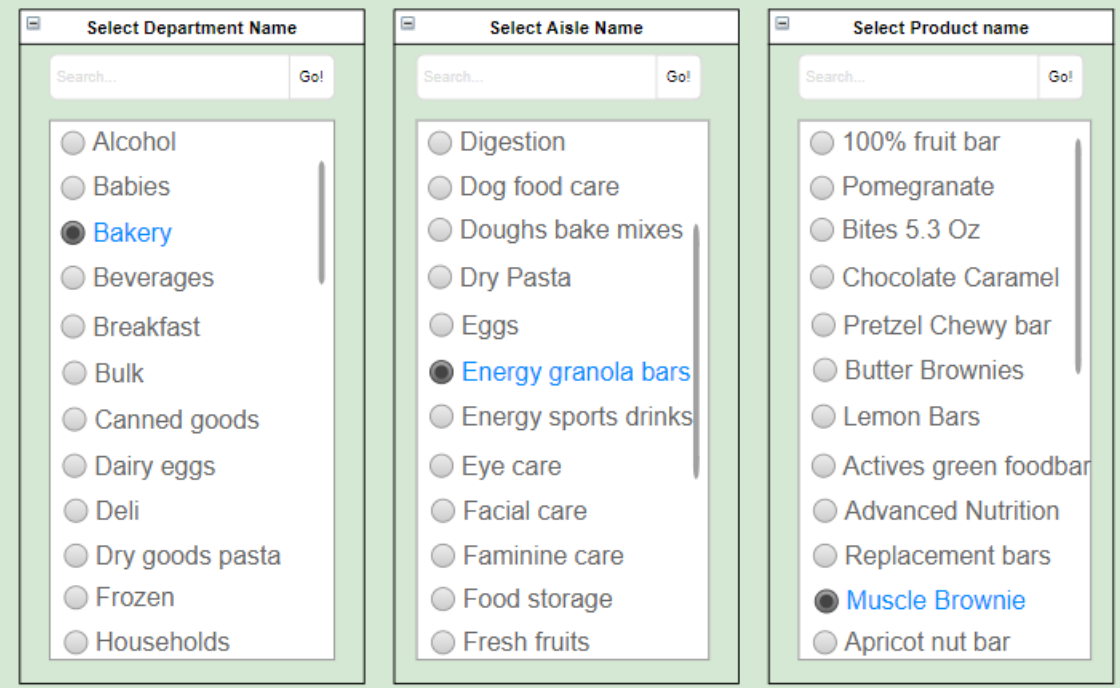

Submit

- Product repurchase probability (Range $\mathbf{0}-\mathbf{1}$ ): $\quad 0.79$

- User propensity score (Range $0-1$ ):

0.6

- User Purchase Intent Classification:

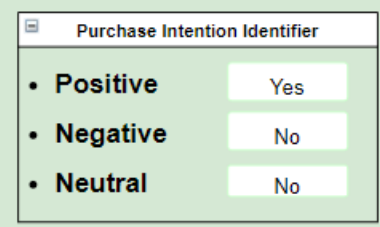

Click to apply coupon type

To interact with the application, first, select the department name, then select the aisle name and then choose the product name for which the repurchase scenario is to be predicted. By hitting the submit button it will predict the re-selling probability score of the product along with user propensity score and purchase intention classification. The predicted output can suggest the conversion rate possibility of that product and hence it 
could be used to select the kind of coupon that would result in the highest profit increase. The second result describes the social media user's propensity range towards the selected product. The final output is the user's purchase intention by gauging publicly available user reviews.

\subsubsection{Potential of the Use case}

In this section, we will discuss the capability of this use case design. The current functionalities of the application provide a good base to the start-up design, but there is a high potential to extend many components of the same.

As discussed in chapter 2, data visualization plays an important role in understanding customer behavior, and to extract the useful knowledge we exploited python code to visually analyze the data. The Salesforce lightning dashboard is a powerful tool for data visualization and presenting it interactively to the user. We can add the graphs of customer behavior patterns directly to the web application page in the future.

Furthermore, the flexible environment of Airtable offers the potential to enhance the data collection and storage layer. While as the application develops, it would be better to store the predicted probabilities of the products and applied coupon details into a relational database to understand the sales and margins for future marketing. Airtable and salesforce platform with Heroku provides a No-SQL database that could be used to store the text data for sentimental analysis.

With high prospects and tractability, we believe this lite and easy-to-use web application can highly benefit an individual retailer by augmenting the available data with inventories, item prices, and coupon details. 


\section{Chapter 7: Evaluation}

The outcomes and results related to different parts of our research procedure are described in this chapter. The content of this chapter is split into three main parts for each research solution. As mentioned earlier, all the processes are implemented in the Python programming language. We evaluate entire algorithms on Microsoft Windows 10 laptop with $1.60 \mathrm{GHz}$ Intel(R)Core(TM)i5-8250U processor, embedded with 16GB physical and 17.4GB virtual memory.

\subsection{Model 1: Purchase probability prediction using historical data}

In this section, we introduce the evaluation part of our first model in detail. From the previous work, we found the most commonly exploited models for purchase probability predictions are Random forest classifier (RFC), Gradient boosting algorithm (In our case, XGB), Neural networks (In our case, CNN).

Recalling the implementation part, we know that we evaluated the model using expanded features based on original data columns. From the results we got in chapter 5, the expanded features prove to generate better outcomes. Before the detailed illustration of evaluations, we begin this part by reminding that we fed our training data to three separate procedural blocks which then combined to generate final output using the Max voting ensemble technique. We assess our model results based on four measures: Functional, Complexity, Efficiency, and Comparative Analysis.

\subsubsection{Functional analysis}

We compare the output of our ensemble model based on the output of each procedural block. We involve an evaluation of how the ensemble technique affects both 
accuracy and efficiency. The normalized confusion matrices of the testing set from each procedural block along with the ensembled output matrix are illustrated in Figure 30.

Figure 30. How does ensemble models affect Confusion matrix results

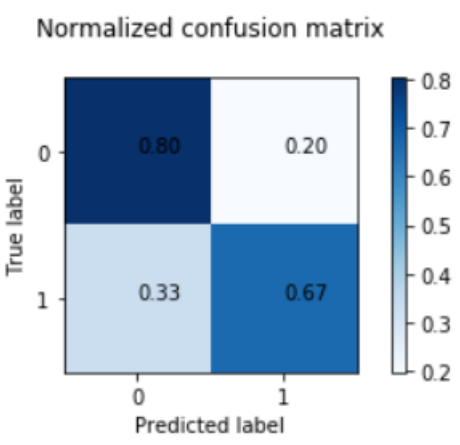

Trained by $\mathrm{RFC}+\mathrm{XGB}$

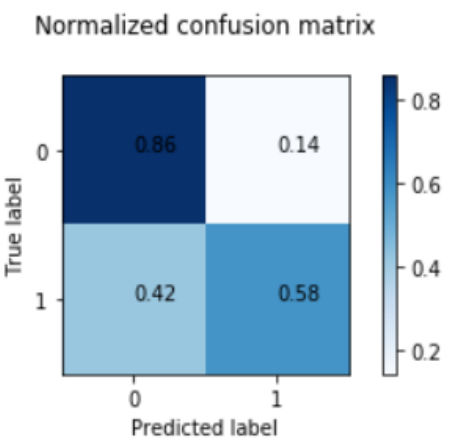

Trained by $\mathrm{CNN}+\mathrm{XGB}$

Normalized confusion matrix

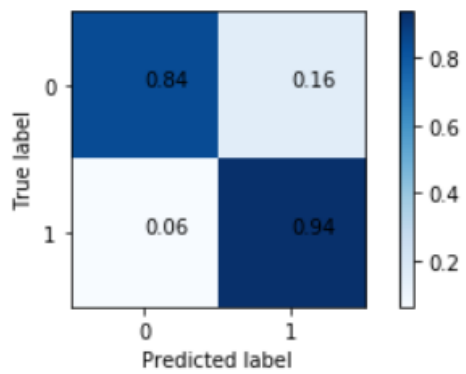

Normalized confusion matrix

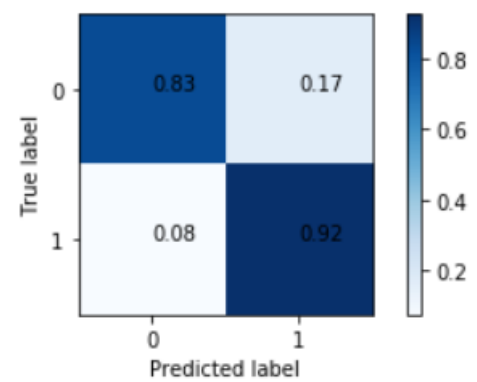

Trained by $\mathrm{CNN}+\mathrm{XGB}+\mathrm{RFC}$

Trained by max voting classifier technique (Segment3)

It shows that all procedural blocks obtained higher than $80 \%$ classification rates for true negative class. Misclassified true positive class is improved when trained in a third procedural block obtaining a 92\% classification rate for true positive class. The overall result illustrated by segment 3 acquires $84 \%$ and $94 \%$ classification rates for true negative and true positive classes, respectively. 
In figure 31, we present the ROC-AUC attained by each procedural block and the final ensemble segment. According to Walter, S. D., 2005, the ROC curve score is directly proportional to the fitness of the model.

Figure 31. How does ensemble models affect the ROC curve

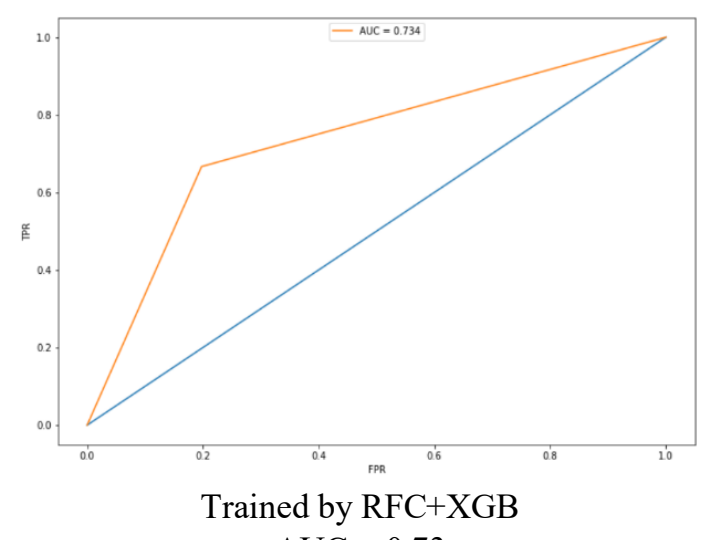

$\mathrm{AUC}=0.73$
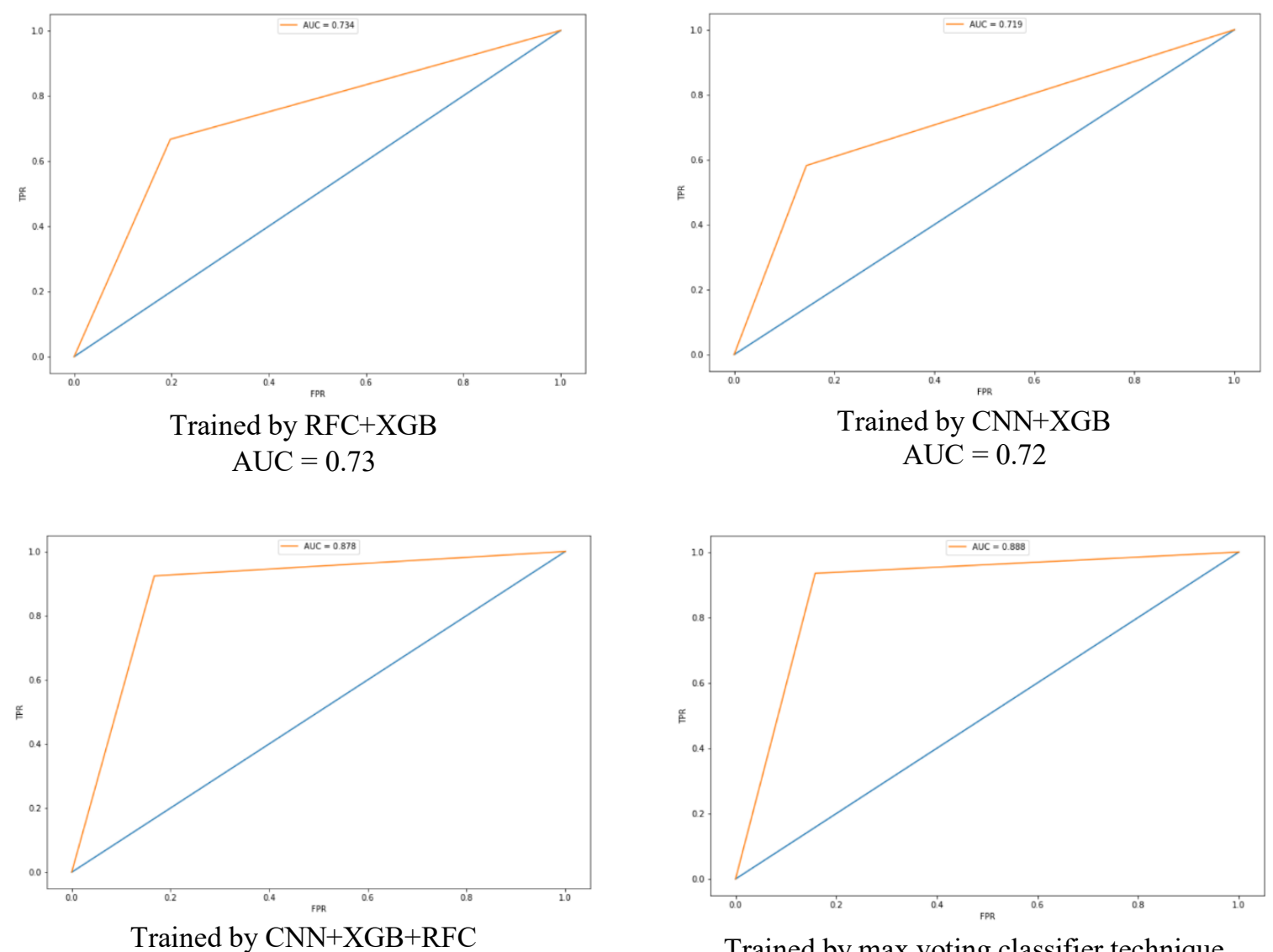

$\mathrm{AUC}=0.87$

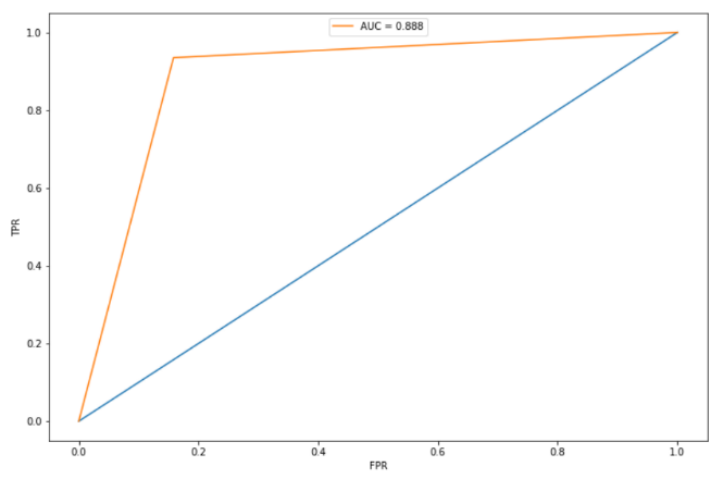

Trained by max voting classifier technique (Segment3)

AUC $=0.88$

The results in the above figure depict the classification accuracy improvement of the model while using the ensemble technique. The minimum area under the curve is obtained by the $\mathrm{CNN}+\mathrm{XGB}$ procedural block, whereas the ensemble classifier calculates the highest AUC score of $88 \%$. 
Table 20. Classification report of model 1

\begin{tabular}{|c|c|c|c|c|}
\hline & Segment-3 & \multicolumn{3}{|c|}{ Segment-2 } \\
\hline & Ensembled result & CNN+XGB+RFC & CNN+XGB & RFC+XGB \\
\hline Accuracy (\%) & 88.84 & 87.82 & 82.87 & 78.90 \\
\hline Precision & 0.85 & 0.84 & 0.30 & 0.26 \\
\hline F1 Score & 0.89 & 0.88 & 0.39 & 0.38 \\
\hline Sensitivity & 0.93 & 0.92 & 0.58 & 0.66 \\
\hline Specificity & 0.84 & 0.83 & 0.85 & 0.80 \\
\hline AUC & 0.88 & 0.87 & 0.72 & 0.73 \\
\hline FPR (fall-out) & 0.15 & 0.16 & 0.14 & 0.19 \\
\hline FNR (miss rate) & 0.06 & 0.07 & 0.41 & 0.33 \\
\hline
\end{tabular}

Table 20 compares other classification results aspects of our model 1 solution. The results were evaluated through the confusion matrix illustrated in Figure 30. After the execution of the classification algorithms, the highest accuracy of $88.84 \%$ is achieved when we put the output of each block together in an ensemble classifier. This signifies that, the ensembled classification approach increases the potential to attain better classification predictions as compared to individual models. Based on these results, the non-consistent behavior of shoppers can be analyzed practically.

\subsubsection{Complexity analysis}

To analyze the complexity of our ensembled model, it must be noted that in the regime where the Convolutional neural network dominates, the other algorithms in the ensembled structure are left out. We employed 1D-CNN utilizing forward propagation.

In the previous work, Kiranyaz, S. et al., 2019, the authors performed an architectural analysis of the 1-dimensional convolutional neural network complexity. The complexity of a neural network is directly proportional to the number of connections between two consecutive CNN layers. At a layer $l$ with $n$ number of nodes, the total number of connections with the previous layer will be $n^{l *} n^{(-1)}$. As mentioned in Kiranyaz, S. et al., 
2019 , theoretically, linear convolution consists of neural weights multiplied to vectors and vector additions from a single connection. Let $n$ be the number of neurons in layer $l$ and $w$ be the size of the kernel vector, the total number of multiplications and additions (ignoring bias addition) for a single layer $l$ defined by the authors is:

$$
\begin{gathered}
n(m u l)^{l}=n^{l *} n^{(l-1)} * S l^{(-1) *}\left(w l^{(l-1)}\right)^{2} \\
n(a d d)^{l}=n^{l *} n^{(l-1) *} S l^{(l-1)}
\end{gathered}
$$

Where, $S l^{(l-1)}$ and $w l^{(l-1)}$ is the vector sizes of the previous layer output. Therefore, for multiple $L$ layers of the network, total computational complexity could be represented as:

$$
\begin{gathered}
T(m u l)^{L}=\sum_{l=1}^{L} n^{l} * n^{(l-1)} * S l^{(l-1)} *\left(w^{(l-1)}\right)^{2} \\
T(a d d)^{L}=\sum_{l=1}^{L} n^{l} * n^{(l-1)} * S l^{(l-1)}
\end{gathered}
$$

According to the above definition, our proposed model is a 2-layers stacked CNN with 3 nodes which depicts $L=2$ and $n=3$ in fully connected dense layer. The equations for multiplication $T(\mathrm{mul})$, nullifies the effect of addition $T($ add $)$ especially when the kernel vector $(w)$ is high.

\subsubsection{Efficiency analysis}

In this section, we illustrate the time spent on training and testing to understand model efficiency, which is further divided according to different segments and blocks mentioned in the model design for simplicity. The time taken by the model divisions is computed and tabulated in Table 21. Since the data set injected in three blocks is the same, which results in equal dataset preparation time $(210.36 \mathrm{~s})$. While the data training time for 
segment 3 is the sum of the overall time taken by three blocks for predicting the individual results.

We also compute the training and testing time for each model division, without a doubt block 3 consume the highest amount of time for model processing as compared to other division.

Table 21. Efficiency analysis of model 1

\begin{tabular}{|c|c|c|c|}
\hline & $\begin{array}{c}\text { Dataset } \\
\text { Preparation } \\
\text { time (s) }\end{array}$ & $\begin{array}{c}\text { Overall training } \\
\text { time (s) }\end{array}$ & Sum (s) \\
\hline $\begin{array}{c}\text { Ensembled } \\
\text { (Segment-3) }\end{array}$ & 5182.07 & 756.18 & 5938.25 \\
\hline $\begin{array}{c}\text { CNN+XGB+RFC } \\
\text { (Block-3) }\end{array}$ & 210.36 & 2570.25 & 2780.61 \\
\hline $\begin{array}{c}\text { CNN+XGB } \\
\text { (Block-2) }\end{array}$ & 210.36 & 1457.13 & 1667.49 \\
\hline $\begin{array}{c}\text { RFC+XGB } \\
\text { (Block-1) }\end{array}$ & 210.36 & 523.61 & 733.97 \\
\hline
\end{tabular}

Our model proves the point raised by Vakili, M. et al., 2020 that neural networks achieve high accuracies at the cost of high execution time.

\subsubsection{Comparative analysis}

Here, we discuss the model performance in comparison to the related works. Considering the distinct nature of each literature including algorithm exploited, dataset used, the purpose of this analysis is to report the performance of acquired results. In the past, an abundance of research is done associated with historical transactional data. The latest recent work that could be comparable to our's to a certain extent is Dou, X., 2020. The author achieved similar model accuracy measurements. They tracked online websites to extract consumer behavior patterns to predict the potential consumer's consumption. The dataset extract involved 12316 unique rows with 17 features. The proposed Catboost 
classification model achieved $88.51 \%$ accuracy with 0.84 recall/sensitivity rate and 0.90 F1-score.

We listed some ensemble-based prediction model performances in table 22, from the comparable metrics, the metric scores of our ensembled model are generally better than other related work. Instead of arbitrarily concluding that our ensemble model outperforms others in related work, we first look into the research work listed in the table below:

Table 22. Comparison of the proposed solution with related work

\begin{tabular}{|c|c|c|c|}
\hline Related work & Dataset & Model & $\operatorname{Accuracy}(\%)$ \\
\hline Tahiri, N. et al., 2019 & $\begin{array}{l}\text { Customer purchase data } \\
\text { from MyGroceryTour.ca } \\
\text { website }\end{array}$ & $\begin{array}{l}\text { RNN + Feedforward } \\
\text { NN }\end{array}$ & $49 \%$ \\
\hline $\begin{array}{l}\text { Adhikari, N. C. D. et } \\
\text { al., } 2017\end{array}$ & $\begin{array}{l}\text { Masked inventory } \\
\text { dataset of } 3 \text { months }\end{array}$ & $\begin{array}{l}\text { An ensemble of time- } \\
\text { series and regression- } \\
\text { based model }\end{array}$ & $71 \%$ \\
\hline $\begin{array}{l}\text { Kaneko, Y., \& Yada, } \\
\text { K., } 2016\end{array}$ & $\begin{array}{l}\text { Grocery product data of } \\
3 \text { years collected from } \\
\text { supermarkets of Japan }\end{array}$ & $\begin{array}{l}\text { Deep learning using L1 } \\
\text { regularization }\end{array}$ & $86 \%$ \\
\hline Dou, X., 2020 & $\begin{array}{l}12316 \text { data records } \\
\text { collected online by } \\
\text { tracking customer's } \\
\text { shopping patterns. }\end{array}$ & CatBoost & $88.51 \%$ \\
\hline $\begin{array}{l}\text { (Proposed solution- } \\
\text { model 1) }\end{array}$ & $\begin{array}{l}2 \text { years transactional } \\
\text { data of Instacart from } \\
\text { Kaggle }\end{array}$ & $\begin{array}{l}\text { Ensemble CNN + XGB } \\
+ \text { RFC }\end{array}$ & $88.84 \%$ \\
\hline
\end{tabular}

Tahiri, N. et al., 2019 also worked on a problem that is very similar to ours. Their main focus was to analyze customer behavior by predicting the purchase patterns. They worked on a dataset from the Canadian grocery store's website MyGroceryTour.ca to evaluate two neural network algorithms (RNN and Feedforward NN) and achieved accuracy and F1-score of $49 \%$ and 0.37 , respectively on the augmented dataset. 
Adhikari, N. C. D. et al., 2017 worked on an anonymous dataset to design their proposed solution using statistical methods aiming to forecast demand of the market. They deploy ensemble techniques consisted of a time series model and a regression-based model and achieved a 71\% accuracy rate. Kaneko, Y. et al., 2016 worked on data collected from variant supermarkets of japan over 3 years period. They exploited the deep learning technique by injecting L1 regularization to analyze fluctuating sales. As a result, they obtained $86 \%$ accuracy with dependency on varying product attributes of supermarkets.

For our proposed solution model 1, we collected sufficient data from Kaggle and applied feature extension to extract in-depth efficient attributes which reasonably explains the effectiveness of our solution. However, Kaneko, Y., \& Yada, K., 2016 chose to analyze the supermarkets in Japan, Adhikari, N. C. D. et al., 2017 performed analysis on 3 months data set, and we obtained the transactional dataset of Instacart, the dataset attributes associated with the retail market of different regions might impact the model performance, which needs further research to validate. Hence, overall, our technique showcased the best performance as in comparison to other mentioned work done on transactional datasets. Our proposed model 1 proved the point raised in Lessmann, S. et al., 2019 that ensemble technique boosts the results of each submodel used in the design.

\subsection{Model 2: Consumer propensity assessment using twitter data}

This section of the chapter includes the evaluation results and findings from the propensity model. Before introducing the discussion on the results obtained, we commence this part by recalling the dynamic nature and non-uniform dimensions of the data that gets scraped through Twitter API. The sentiment tagging is done using the VADER sentiment analyzer as explained in chapter 6 . The distribution percentage of all the tweets in three 
sentiment categories is illustrated in figure 32 . The maximum tweets belong to the positive category with a rate of $46 \%$. Following, we got $40 \%$ and $14 \%$ tweets in neutral and negative sentiment categories, respectively. The output in figure 32 depicts the level of confidence in positive tweets is higher than in neutral and negative tweets.

Figure 32. Distribution of sentiments across all Tweets

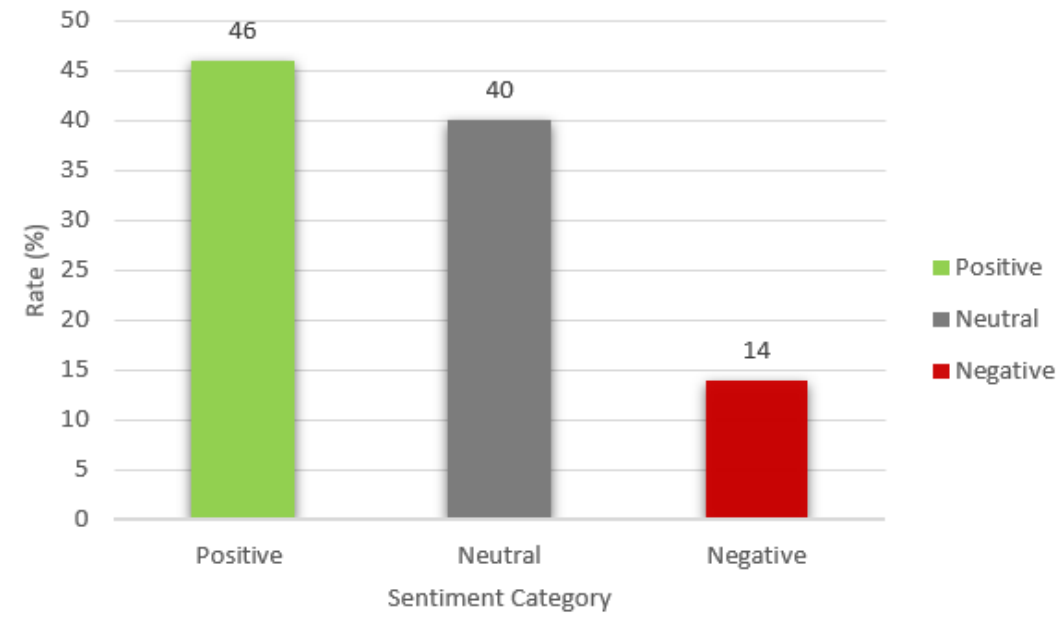

The purpose of this section is to first introduce the functional outcomes of our experimental setup to predict the sentiments of the twitter users. Next, we analyze the complexity of each algorithm employed for the prediction, followed by the analysis of the model efficiency. Lastly, we compare our model results with other existing work done in the same field.

\subsubsection{Functional analysis}

The prediction results are obtained by employing Random forest (RFC) and Support Vector Machine (SVM) as the text classification algorithms. The system performance is evaluated using different classification measures which are Accuracy, Precision, F1-score and sensitivity. 
After the execution of the random forest and support vector machine, the evaluation of the results via the confusion matrix is illustrated in Figure 33.

Figure 33. How do text classification models affect Evaluation results

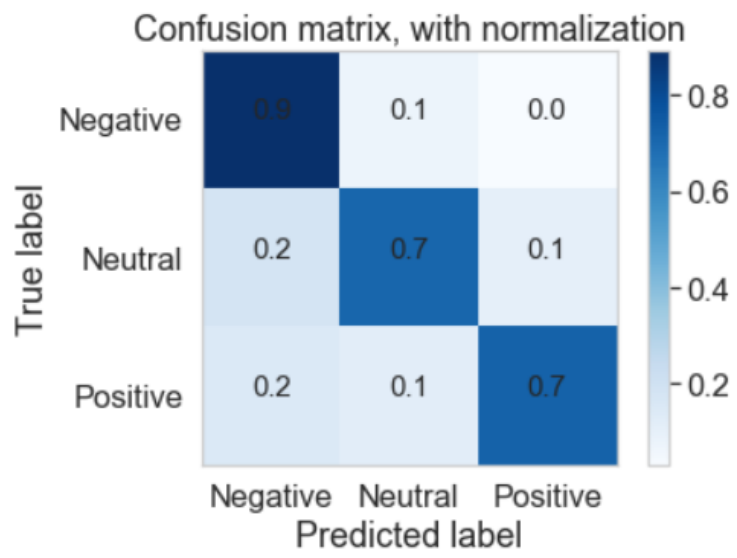

Random forest text classifier

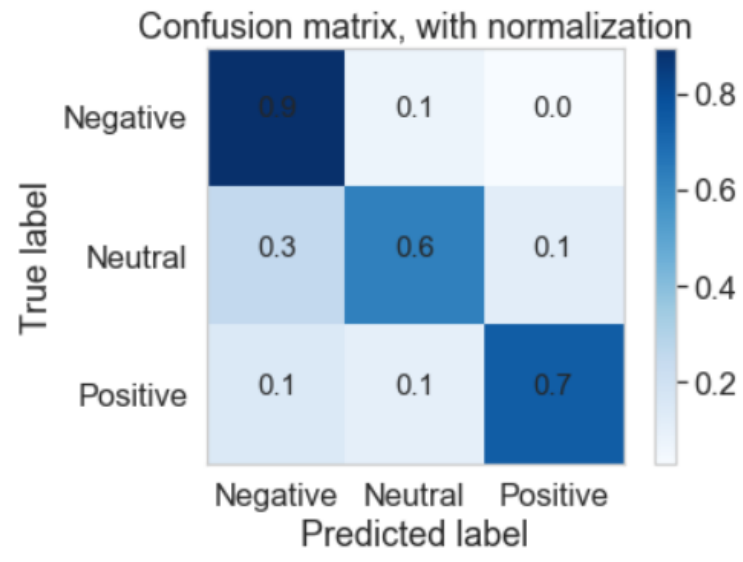

Support vector machine text classifier

For both RFC and SVM text classifiers, the accuracy of $77.78 \%$ and $75.62 \%$ were obtained, respectively. The classification report in Table 23 shows a comparison between both the text classifiers and reveals other measures such as an F1 score of 0.78 for RFC and 0.75 for SVM. Throughout the model assessment, the constraint of non-uniform data hampered the capacity of the work, yet best efforts have been observed to accurately classify the sentiment categories.

Table 23. Classification report of model 2

\begin{tabular}{|c|c|c|}
\hline & RFC & SVM \\
\hline Accuracy (\%) & 77.78 & 75.62 \\
\hline Precision & 0.77 & 0.77 \\
\hline F1 Score & 0.78 & 0.75 \\
\hline Sensitivity & 0.78 & 0.76 \\
\hline
\end{tabular}


Following the text classification, we gauged the attributes of the tweets in terms of products in discussion and user location to assess the propensity level. Figure 34 demonstrates the popularity of each product among twitter users in Canada. Maximum of the users displayed positive opinions towards Blueberries whereas no user displayed negative sentiment regarding Strawberries. Hence, the forthcoming buying fluctuation of each product could be determined with respect to short-term social media by analyzing user propensity levels.

Figure 34. Propensity of each product

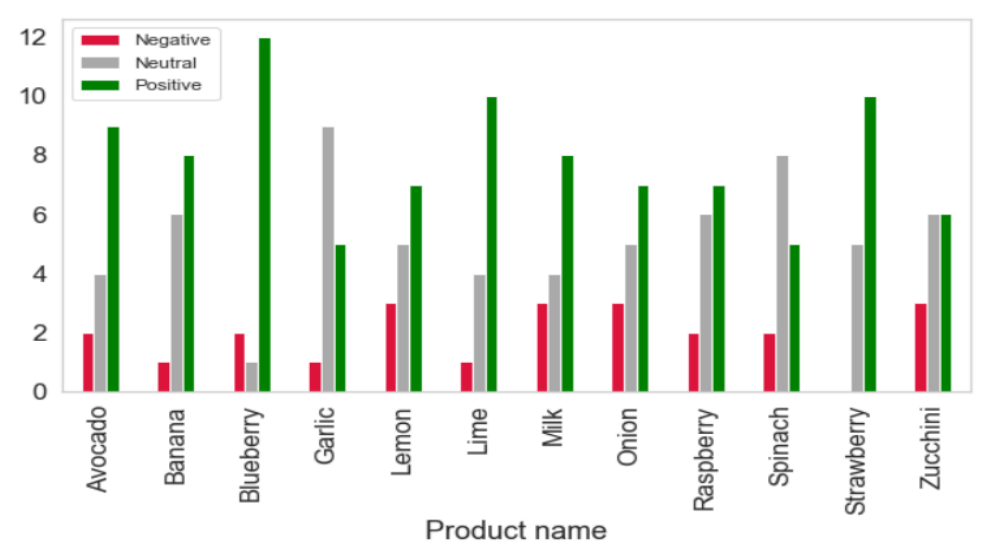

The next graph in figure 35 depicts the number of tweets posted in different cities across Canada. As shown, the maximum number of positive tweets has been reported in Toronto followed by Edmonton and Vancouver whereas Vancouver shows no negative tweet, and the highest number of negative tweet content was been recorded in Toronto. 
Figure 35. User Propensity for top products per location

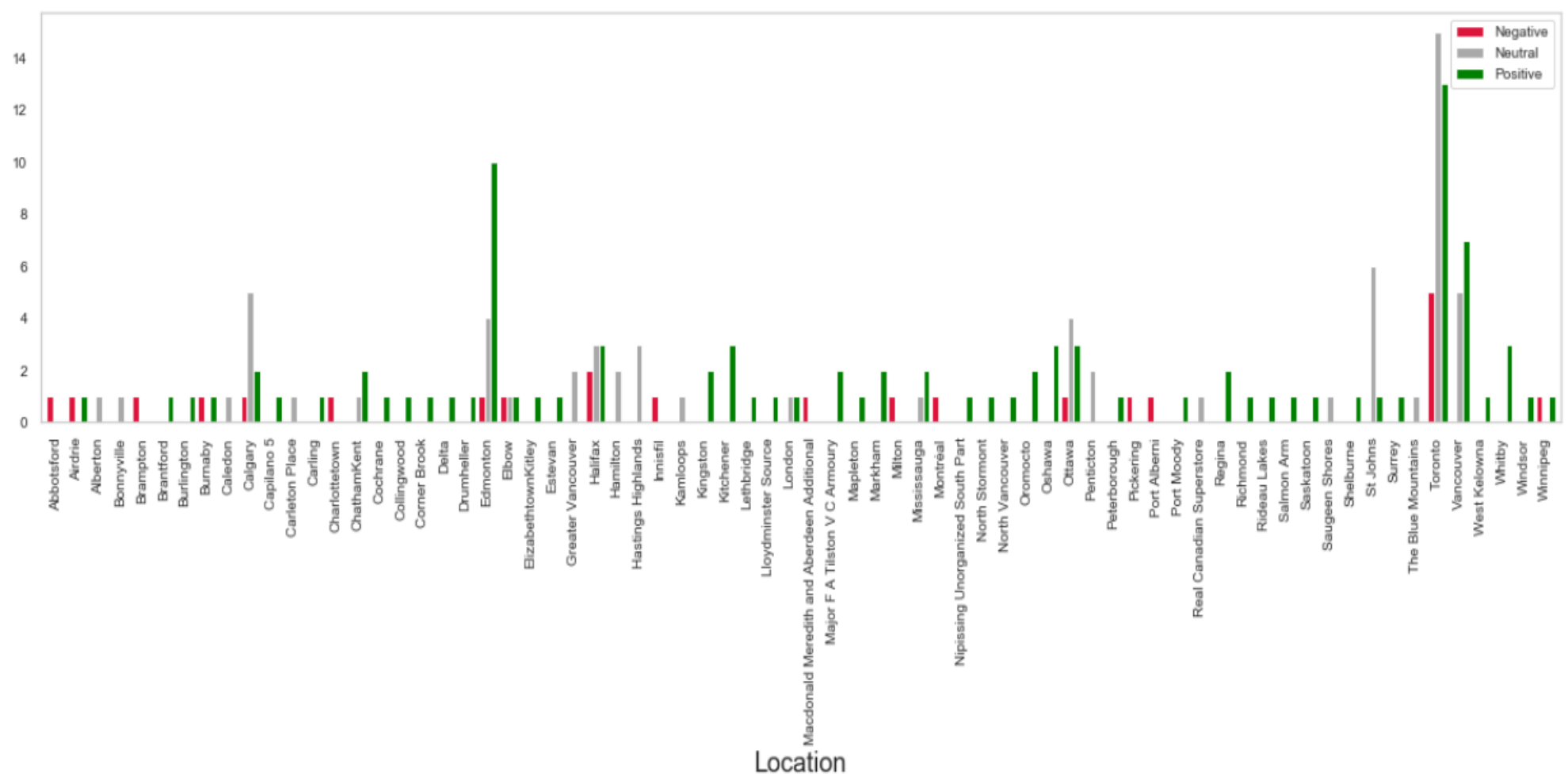

We also evaluated user propensity for a specific product according to the location of the tweet. Figure 36 depicts the sentimental distribution of tweets across cities of Canada. We provided "Zucchini" as an input to be evaluated by the function created in python language for visualization.

Being the most active city of twitter users as illustrated in the Figure above, zucchini had neutral sentiment impact in Toronto. 
Figure 36. User Propensity for "Zucchini”" per location

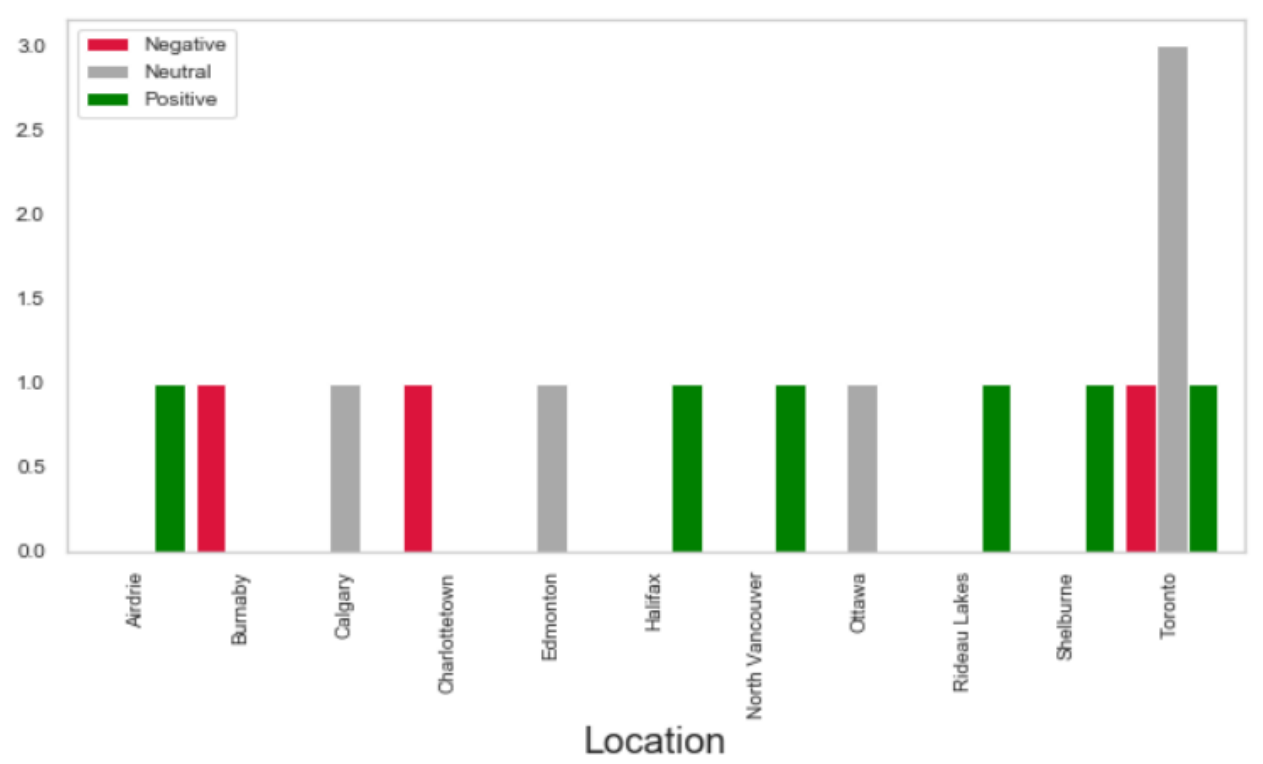

Similarly, we evaluated the propensity level for each most re-purchased product of Instacart at a specific location. In Figure 37, we evaluated twitter activity for Toronto. The maximum positive sentiments were posted on Limes followed by Banana and Spinach. Onion and Zucchini were categorized most for neutral user sentiments.

Figure 37. User Propensity for top products at "Toronto" location

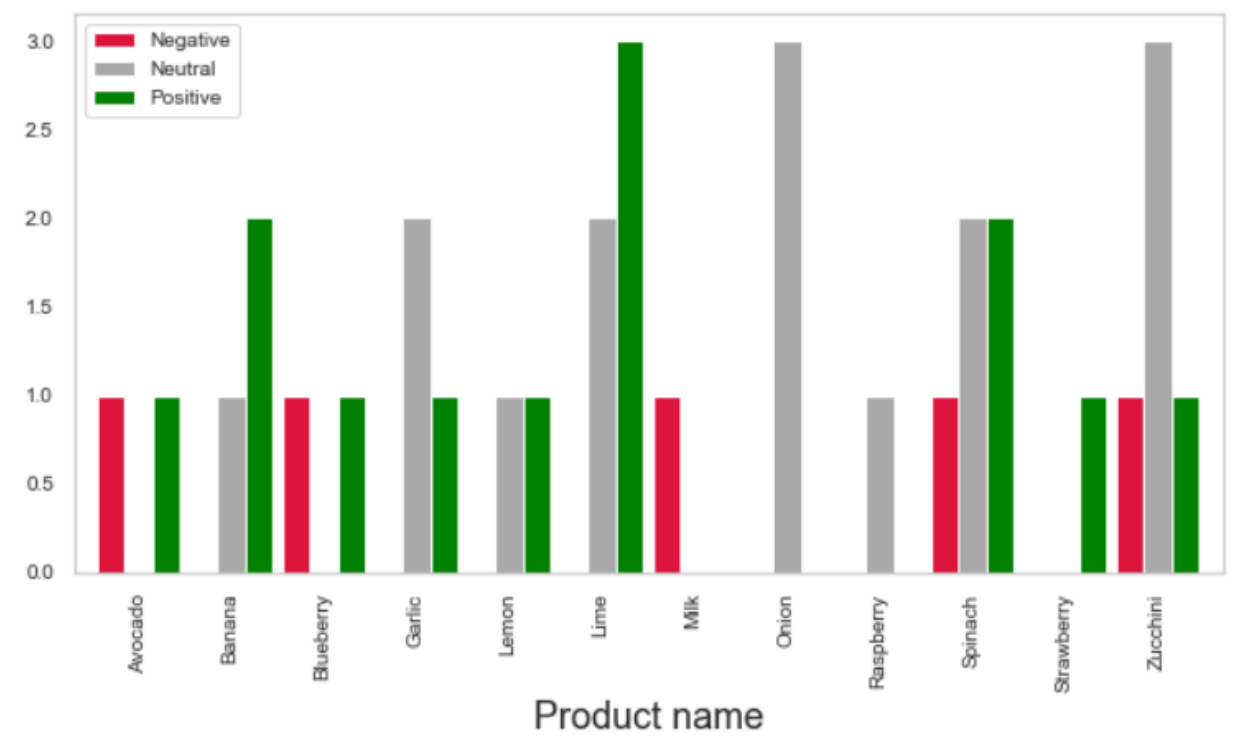


To conclude, we can provide insightful knowledge by promoting visual representations of our data. The visual charts organize the data in such a way that it can help retailers to pinpoint the prospecting market demand at a glance.

\subsubsection{Complexity analysis}

In this part, we discuss the complexity of our propensity assessment model which is build using Random forest and support vector machines. To illustrate the computational complexity of our model, let us denote the number of training samples as $n, v$ as the number of support vectors, and $s$ be the dimension of the data. For the SVM classifier, according to Abdiansah, A., \& Wardoyo, $R$., 2015, the time complexity for $n$ data points is $\mathrm{T}(n)=$ $\mathrm{O}\left(n^{2}\right)$ at the training time, and $\mathrm{T}(n)=\mathrm{O}\left(v^{*} s\right)$ during the test time. The space complexity for $n$ data points is computed as $\mathrm{S}(n)=\mathrm{O}(v)$ to store $v$ vectors. The complexity cost for SVM could increase with the fluctuation in the number of training samples while scraping data.

According to the definitions of unpruned decision trees (Louppe, G., 2014), the time complexity for our random forest model is $\mathrm{T}(n)=\mathrm{O}\left(n^{*} \log (n)^{*} s^{*} k\right)$ where $k$ is the number of decision trees and $s$ is the data dimension. To save the $k$ number of trees with depth $d$, space complexity is calculated as $\mathrm{S}(n)=\mathrm{O}\left(d^{*} k\right)$.

\subsubsection{Efficiency analysis}

In this section, we discuss the execution time taken by both algorithms utilized in model 2. To begin with, we compute the data preparation time which includes data scraping, cleaning, sentiment tagging. This step of dataset preparation is equally run for both algorithms; hence they utilize comparable execution time. 
Table 24. Efficiency analysis of model 2

\begin{tabular}{|c|c|c|c|}
\hline & $\begin{array}{c}\text { Dataset } \\
\text { Preparation } \\
\text { time (s) }\end{array}$ & $\begin{array}{c}\text { Model training } \\
\text { time (s) }\end{array}$ & Sum (s) \\
\hline RFC & 56.30 & 35.11 & 91.41 \\
\hline SVM & 55.94 & 36.95 & 92.89 \\
\hline
\end{tabular}

We train baseline RFC and SVM algorithms, which again spend the analogous time of execution. Therefore, we can conclude that in terms of efficiency, both algorithms are on par with each other.

\subsubsection{Comparative analysis}

In this portion, we discuss the results of related works as compared to our solution model 2. While performing text classification, all the related work listed in table 25 performed distinct feature preprocessing and exploited different datasets, which results in varying functional measurements. The research works done in the field of twitter data analysis listed in Table 25 are by some means comparable to our work done.

Kumari, P., \& Haider, M. T. U., 2020 employed LSTM for sentiment prediction over tweets based on the Aadhaar security process followed in India. They compared their results with existing state-of-the-art algorithms, their proposed model obtained $85.3 \%$ model accuracy which is higher in comparison to other existing algorithms.

Table 25. Comparison of the proposed solution with related work

\begin{tabular}{|c|c|c|c|}
\hline Related work & $\begin{array}{l}\text { Twitter Data } \\
\text { explanation }\end{array}$ & Model approach & Accuracy $(\%)$ \\
\hline $\begin{array}{l}\text { Kumari, P., \& Haider, } \\
\text { M. T. U., } 2020\end{array}$ & $\begin{array}{l}\text { Aadhaar cart-related } \\
\text { tweets collected using } \\
\text { Tweepy API }\end{array}$ & $\begin{array}{l}\text { Hybrid LSTM, Naïve } \\
\text { Bayes, Logistic } \\
\text { regression, SVM }\end{array}$ & $\begin{array}{c}\text { Score of } \mathbf{8 5 . 3 \%}, 71.4 \%, \\
80.6 \% \text { and } 81.3 \%, \\
\text { respectively }\end{array}$ \\
\hline $\begin{array}{l}\text { Da Silva, N. F. et al., } \\
2014\end{array}$ & $\begin{array}{l}\text { Sanders, Stanford, } \\
\text { Obama-McCain Debate } \\
\text { and Health care reform }\end{array}$ & $\begin{array}{l}\text { Ensemble SVM, NB, } \\
\text { RF and LR }\end{array}$ & $\begin{array}{c}\text { Score of } 76.99 \%, \\
76.81 \%, 84.89 \% \text { and } \\
81.06 \% \text { respectively }\end{array}$ \\
\hline
\end{tabular}




\begin{tabular}{|l|l|l|c|}
\hline $\begin{array}{l}\text { Joshi, R., \& } \\
\text { Tekchandani, R. 2016 }\end{array}$ & $\begin{array}{l}\text { Movies reviews-based } \\
\text { tweets }\end{array}$ & $\begin{array}{l}\text { SVM, NB, Maximum } \\
\text { Entropy }\end{array}$ & $\begin{array}{l}\text { Score of } 84 \%, 70 \% \text { and } \\
74 \%, \text { respectively }\end{array}$ \\
\hline $\begin{array}{l}\text { Liang, P. W., \& Dai, B. } \\
\text { R. } 2013\end{array}$ & $\begin{array}{l}\text { Tweets of three } \\
\text { categories (Camera, } \\
\text { mobile phone, movie) } \\
\text { using Twitter API }\end{array}$ & $\begin{array}{l}\text { Novel Unigram Model } \\
\text { and Opinion Miner }\end{array}$ & $\begin{array}{l}\text { Score of } 67.58 \% \text { and } \\
70.39 \%, \text { respectively }\end{array}$ \\
\hline $\begin{array}{l}\text { (Proposed solution- } \\
\text { model 2) }\end{array}$ & $\begin{array}{l}\text { Short-term real-time } \\
\text { publicly available } \\
\text { tweets }\end{array}$ & RFC and SVM & $\begin{array}{l}77.78 \% \text { and } 75.62 \%, \\
\text { respectively }\end{array}$ \\
\hline
\end{tabular}

By looking into the dataset and model approach column of table 25, Da Silva, $N$. F. et al., 2014 introduced an ensemble approach to target the public sentiments towards consumer brands. The author leveraged the ensemble methodology on Multinomial Naïve Bayes, SVM, Random Forest, and Logistic regression algorithms. They implemented their proposed solution on four different datasets (mentioned in table 25) and evaluated model accuracy for each of them, obtaining highest accuracy of $84.89 \%$ on Sanders dataset, whereas Liang, P. W., \& Dai, B. R. 2013 introduced their novel method to analyze sentiments of twitter users based on three distinct categories. The data collection technique used by the author is similar to ours. Their integrated machine learning approach, Unigram model, and Opinion miner achieved 67.585 and $70.39 \%$ accuracy.

The most comparable methodology strategy was followed in Joshi, R., \& Tekchandani, R. 2016. The author performed sentiment analysis on movies related tweets by exploiting SVM, Naïve Bayes, and Maximum entropy classifiers, to achieve $84 \%, 70 \%$, and $74 \%$ accuracy for respective models. For our model 2 , we adopted fundamental machine learning algorithms(RFC and SVM), to evaluate tweets based on Instacart products, and achieved $77.78 \%$ accuracy. To conclude, our model proficiently analyzes the demographic placements of prospecting customers with regards to the product with 
substandard classification results in comparison, which could be due to the fluctuation in the real-time data which leads to difficulty in the aggregation of individual data points.

\subsection{Model 3: Purchase intention detection based on online reviews}

Here in this part, we discuss the performance outcomes of our third model solution which is based on identifying the user purchase intent. We utilize five algorithms to establish an evaluation comparison of our processed dataset. We inaugurate the model performance analysis by recalling that each algorithm is evaluated thrice which focuses on binary classification for three distinct purchase intent labels: Positive, Negative, and Unknown.

We input the pre-processing data for training and testing the models. In what follows, we first detail the functional results of the experiments. Following we discuss the complexity analysis done on each algorithm design and then we evaluate the model efficiency in terms of time taken. We wrap the model evaluation by discussing the comparative analysis of our proposed solution with other related work.

\subsubsection{Functional analysis}

This section discusses experimental results on predictions produced by our customized algorithms. For metrics consistency with other solution models, we calculated accuracy, F1-score, precision, recall, and ROC-Area under the curve as evaluation metrics. Due to high-class imbalance, F1-score is considered the primary metric of discussion, which combines harmonic mean of precision and recalls into a single metric.

$$
F 1=\frac{2 * \text { precision } * \text { recall }}{\text { precision }+ \text { recall }}
$$


As we follow Atouati, S. et al., 2020, we perform 10-fold cross-validation over the manually labeled data for each intent label. For example, in the case of predicting negative purchase intent, we only consider $n p i$ feature label by overlooking the other two (ppi and upi) and vice versa. Please note that while evaluating LightGBM and NeighLOR algorithms, we applied both, our customized loss function and Atouati, S. et al., 2020's tailored cost function. While for LSTM, we only use Atouati, S. et al., 2020's cost function disregarding loss function, whereas we employ logistic regression and CNN without any tailoring. The results are shown in Table 26.

Table 26. Classification report of model 3

\begin{tabular}{|c|c|c|c|c|c|c|c|c|c|c|c|c|c|c|c|}
\hline \multirow{3}{*}{$\begin{array}{l}\text { Evaluatio } \\
\text { n metrics }\end{array}$} & \multicolumn{6}{|c|}{$\begin{array}{l}\text { Using Atouati, } S . \text { et al., 2020's cost and our } \\
\text { customized loss function }\end{array}$} & \multirow{2}{*}{\multicolumn{3}{|c|}{$\begin{array}{c}\begin{array}{c}\text { Using Atouati, S. et al., } \\
2020 \text { 's cost function } \\
\text { only }\end{array} \\
\text { LSTM }\end{array}$}} & \multicolumn{6}{|c|}{ Disregarding both cost and loss functions } \\
\hline & \multicolumn{3}{|c|}{ Light GBM } & \multicolumn{3}{|c|}{ NeighLOR } & & & & \multicolumn{3}{|c|}{ Logistic regression } & \multicolumn{3}{|c|}{ CNN } \\
\hline & NPI & PPI & UPI & NPI & PPI & UPI & NPI & PPI & UPI & NPI & PPI & UPI & NPI & PPI & UPI \\
\hline $\begin{array}{l}\text { F1 Score } \\
\text { (\%) }\end{array}$ & 94.17 & 92.2 & 87.4 & 95.7 & 94.4 & 87.02 & 94.04 & 92.24 & 86.40 & 48.05 & 50.83 & 96.2 & 30.65 & 51.7 & 86.4 \\
\hline $\begin{array}{l}\text { Precision } \\
(\%)\end{array}$ & 92.3 & 89.8 & 90.2 & 96.02 & 94.2 & 85.8 & 92.17 & 89.83 & 82.42 & 53.9 & 52.3 & 95.8 & 15.9 & 27.2 & 82.4 \\
\hline $\begin{array}{c}\text { Recall } \\
(\%)\end{array}$ & 96.08 & $\begin{array}{l}94.7 \\
9\end{array}$ & 85.7 & 96.31 & 94.9 & 90.6 & 96.0 & 94.77 & 90.78 & 51.3 & 52.6 & 96.7 & 39.9 & 52.2 & 90.7 \\
\hline $\begin{array}{l}\text { ROC- } \\
\text { AUC } \\
(\%)\end{array}$ & 80.7 & 80.9 & 80.5 & 90.08 & 89.9 & 54.01 & 83.4 & 78.7 & 81.3 & 94.08 & 88.7 & 86.02 & 50.4 & 50.0 & 50.0 \\
\hline $\begin{array}{l}\text { Accurac } \\
\text { y (\%) }\end{array}$ & 96.08 & $\begin{array}{l}94.7 \\
9\end{array}$ & 85.7 & 96.31 & 94.9 & 90.6 & 96.0 & 94.77 & 90.78 & 95.8 & 95.4 & 93.2 & 39.9 & 52.1 & 90.7 \\
\hline
\end{tabular}

We find that tailored LightGBM and NeighLoR achieved remarkably similar results for all three intent classifications and they outperform other models followed by LSTM in terms of F1-score. The competitive results of LSTM are due to the fact that Atouati, S. et $a l$., 2020's approach to tailoring the cost function is inherited from the recurrent neural network. In contrast, disappointing results of $\mathrm{CNN}$ might be due to the fact that the quantity of labeled data suppressed the potentials of the algorithm. 
While studying the overall performance of the experiments, the ROC_AUC score is taken into consideration. From table 26, we can observe that most consistent and simillar results are acquired by the LightGBM model for all three intent label classifications in terms of all classification measurements. Hence, the results depict that combining our tailored loss function with Atouati, S. et al., 2020 approach yields better performance as compared to metric results obtained by algorithm used by Atouati, S. et al., 2020.

We evaluate each intent label individually based on four metrics: F1-score, precision, recall, and ROC-AUC score. Figure 38 depicts the results of negative purchase intent classifiers. The highest F1-score is achieved by the NeighLoR classifier and results of LightGBM are at par with it, $95.7 \%$ and $94.17 \%$, respectively.

Figure 38. Negative Purchase intent assessment

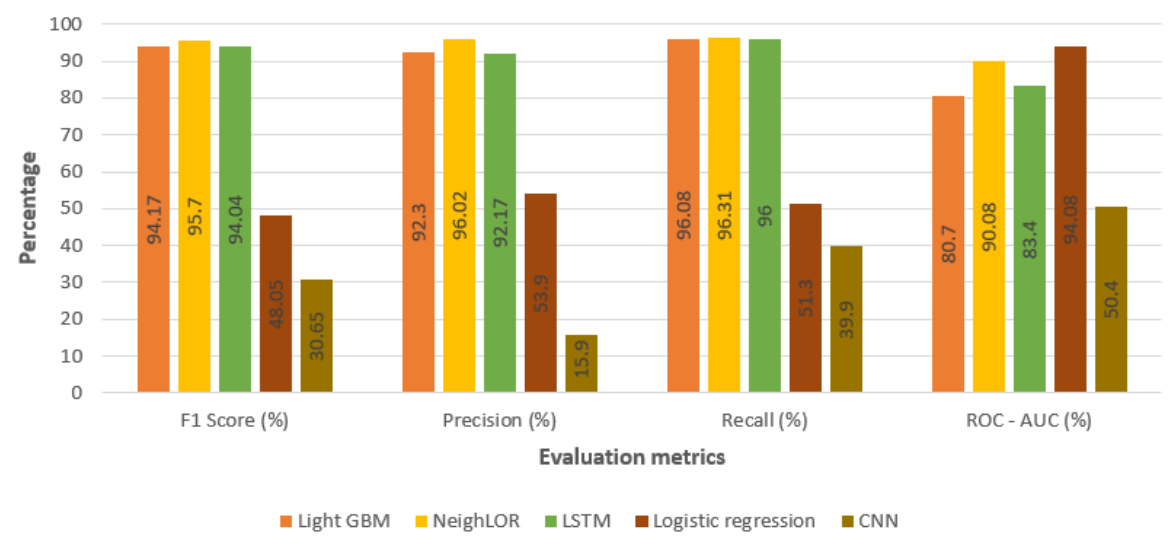

The positive intention detection classification results follow a similar trend. The maximum metric scores are achieved by NeighLoR followed by LightGBM and LSTM. Whereas Logistic regression model obtained the lowest F1-score and minimum precision and recall are manifested by the $\mathrm{CNN}$ classifier. 
Figure 39. Positive Purchase intent assessment

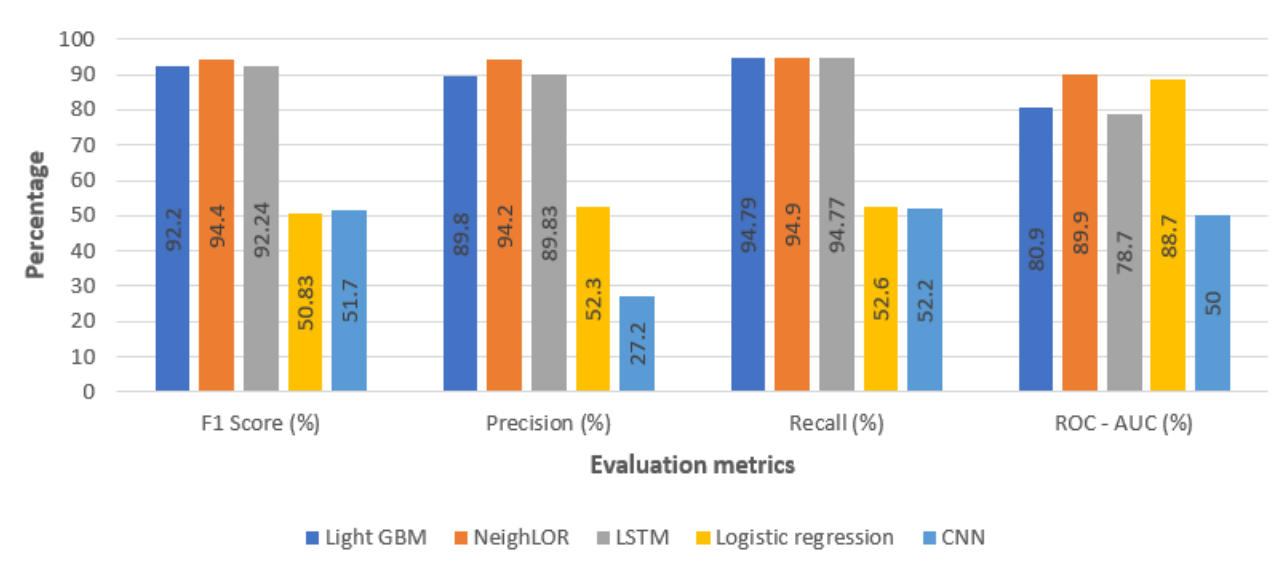

Surprisingly, the results of Logistic regression for unknown purchase classification are overriding other algorithms as it achieved 96.2\% F1-score, followed by LightGBM, which performed fairly well with $87.7 \%$ F1-score. Whereas NeighLoR achieved substandard results. Interestingly, while classifying unknown purchase intent, both LSTM and CNN classifiers exhibited similar results, whereas a huge difference can be seen in the case of the other two labels (positive and negative).

Figure 40. Unknown Purchase intent assessment

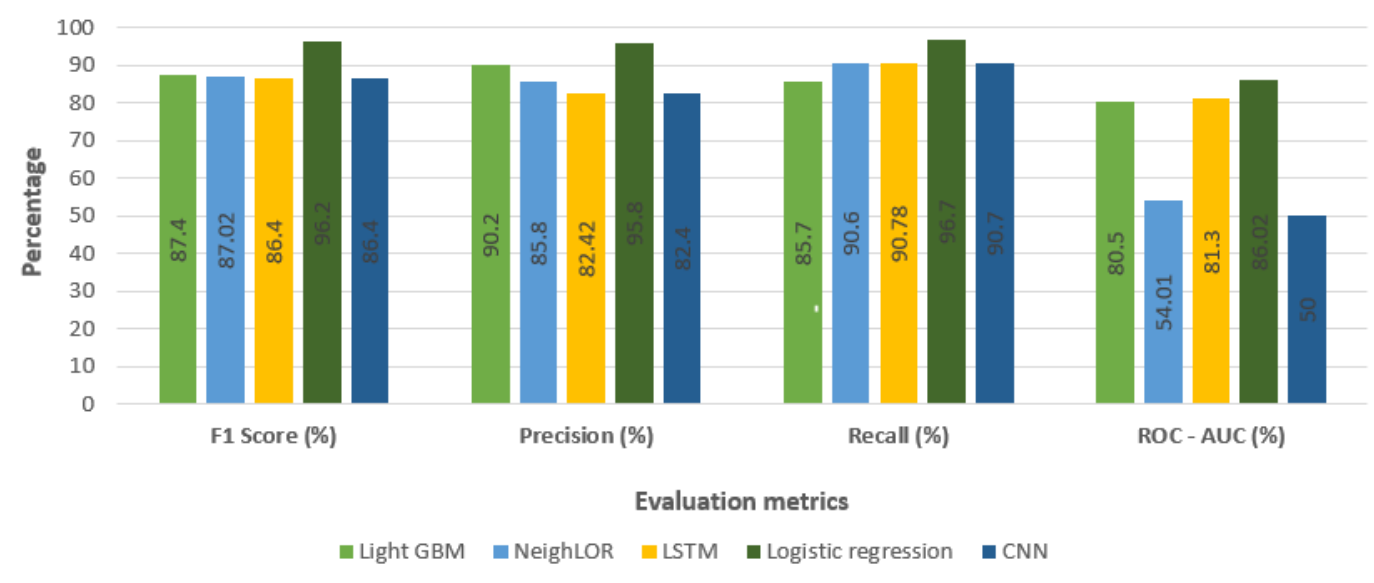


In nutshell, our personalized loss function approach blended with Atouati, S. et al., 2020 works finest for positive and negative purchase intent classification and dominated other algorithms in every aspect of measurement. Considering the variations in metric results and overall model results, LightGBM verified to be the most coherent model for the intent identification problem. However, the reason for the distinct trend shown in the case of negative purchase intent classification might be due to the dominance of word dependency parsing. We concentrated on parsing the words reflecting negation and affirmation features while rest were considered unknown, thus demanding further consideration.

\subsubsection{Complexity analysis}

This section focuses on inferring the complexity analysis of each algorithm utilized for the experiments of model 3. To begin with, we discuss the nature of our first algorithm: LightGBM. Ke, G. et al., 2017 introduced the novel technique of implementing Gradientbased One-Side Sampling (GOSS) and Exclusive Feature Bundling (EFB) in Gradient Boosting Decision Tree named LightGBM. Hence, the core cost is based on learning the decision trees.

The author mentions that the complexity of the algorithm lies in finding the best split points on the data labels to build the histograms, which evaluates to be the order of $n$ $* d$, where $\mathrm{n}$ is the number of data points and $\mathrm{d}$ is the number of data dimensions. In our case, dimension $d$ for each intent label is defined in the form of a matrix $M^{h_{r} * f}$ Where $h_{r}$ represents the number of neighborhoods constructed for review $r, f$ represents the features. 
The cost of matrix calculation is $\mathrm{O}\left(n^{2}\right)$, therefore the overall cost for training is $\mathrm{O}\left(n^{*} n^{2}\right)=\mathrm{O}\left(n^{3}\right)$

The next algorithm in consideration for complexity evaluation is tailored NeighLoR, which is an extension to logistic regression. Li, J. et al., 2011 defined the complexity of logistic regression algorithm with gradient-based optimization as $\mathrm{O}((d+1) c n)$, where $\mathrm{d}$ is defined as data dimension, $\mathrm{n}$ is the number of data points, $c$ is the number of classes under prediction. As we determine the binary classification, the value of $c=1$. To save the $d$ dimensional data where $\mathrm{d}$ is in the form of $2 * 2$ matrix, the algorithmic space complexity is calculated as $\mathrm{O}(\mathrm{d})$. Similarly, for a simple logistic regression model, the data has a linear dimension, thus reducing the overall complexity cost.

Next, for convolutional neural network cost analysis on linear textual data converted into matrix form, we recall architectural analysis from Kiranyaz, S. et al., 2019 discussed in section 7.1.2 At a layer $l$ with $n$ number of nodes, the total number of connections with the previous layer will be $n^{l *} n^{(l-1)}$, Let $v$ be the vector size and $w$ be the weight assigned to the kernel, the cost for the total number of multiplications and additions (ignoring bias addition) for multiple layers where $l=(1 . . n)$ defined by the authors is:

$$
\begin{array}{r}
T(m u l)^{l}=\sum_{l=1}^{L} n^{l} * n^{(l-1)} * S^{(l-1)} *\left(w^{(l-1)}\right)^{2} \\
T(\text { add })^{l}=\sum_{l=1}^{L} n^{l} * n^{(l-1)} * S^{(l-1)}
\end{array}
$$

Where $T(a d d)$ is insignificant as compared to $T(m u l)$. For the solution model 3 , we deploy 2-layers sequentially stacked CNN with 3 nodes which depict $L=2$ and $n=3$. There total time complexity :

$$
\mathrm{T}=T(m u l)^{l}
$$


While performing matrix calculations, it is assumed that the cost is quadratic order of $n$. Therefore, $T(m u l)=n^{l} * n^{3}=O\left(n^{*} n^{3}\right)=O\left(n^{4}\right)$.

Lastly, we evaluate the complexity of the recurrent neural network applied. For analyzing the cost of the LSTM model, we refer to Hochreiter, S., \& Schmidhuber, J. 1997. The author defined that cost of LSTM is local in space and time, which means complexity per weight is $\mathrm{O}(1)$ and space complexity is unaffected by input length. Hence, the overall complexity is only dependent on the number of weights defined, $\mathrm{O}(w)$.

\subsubsection{Efficiency analysis}

Here we compare the algorithms used in model 3 in terms of time efficiency shown at different levels of model building. To begin with, the data collected for each model is the same, hence, the first input column of table 27 represents the same digits for each algorithm. We did not consider the time taken by the manual labeling task. However, we compute the time taken for feature selection which happens to be the same for each intent label irrespective of the word-intent relationship. Hence, we mention the overall data preprocessing time for each algorithm.

Table 27. Efficiency analysis of model 3

\begin{tabular}{|c|c|c|c|c|}
\hline & $\begin{array}{c}\text { Dataset } \\
\text { collection } \\
\text { time (s) }\end{array}$ & $\begin{array}{c}\text { Data pre-processing } \\
\text { time (s) }\end{array}$ & $\begin{array}{c}\text { Model training } \\
\text { time (s) }\end{array}$ & Sum (s) \\
\hline LightGBM & 83.23 & 64.55 & 94.48 & 242.26 \\
\hline NeighLoR & 83.23 & 65.52 & 1010.30 & 1159.05 \\
\hline LSTM & 83.23 & 66.83 & 719.51 & 869.57 \\
\hline Logistic regression & 83.23 & 68.76 & 0.73 & 153.72 \\
\hline CNN & 83.23 & 65.74 & 246.53 & 395.50 \\
\hline
\end{tabular}

After calculating the time taken at every step by each algorithm and considering the function and complexity analysis, we conclude that the customized LightGBM utilizing 
neighborhood construction of Atouati, S. et al., 2020 proves the superiority and dominance over other models.

\subsubsection{Comparative analysis}

The discussion in this section initiates a comparison of our model 3 experiment results with other previous work concentrated on a similar problem. Primarily, we assess our functional results with Atouati, S. et al., 2020. The author introduced a novel extension of the logistic regression model by defining neighborhoods for intent reflecting words aiming to detect negative intent in tweets. To evaluate the results, they collected two datasets related to British Airways and achieved an F1-score of $80.79 \%$. We followed their approach to build our tailored version, which outperformed the results of Atouati, S. et al., 2020 by significant measures. The following table consists highlights of similar work done:

Table 28. Comparison of the proposed solution with related work

\begin{tabular}{|c|c|c|c|}
\hline Related work & Data explanation & Model approach & Metrics and results \\
\hline Atouati, S. et al., 2020 & $\begin{array}{l}\text { Twitter data on British } \\
\text { Airways }\end{array}$ & $\begin{array}{l}\text { neighbor based on } \\
\text { logistic regression }\end{array}$ & F1-score: $80.79 \%$ \\
\hline Gupta, V. et al., 2014 & $\begin{array}{l}\text { Quora and Yahoo! } \\
\text { Answers datasets }\end{array}$ & $\begin{array}{l}\text { Support vector } \\
\text { machine }\end{array}$ & $\begin{array}{l}\text { The area under the curve: } \\
\text { Quora dataset }=0.93 \text {, } \\
\text { Yahoo! Answers dataset }= \\
0.89\end{array}$ \\
\hline Ding, X. et al., 2015 & $\begin{array}{l}\text { large-scale microblog } \\
\text { corpus from Sina } \\
\text { Weibo related to kids } \\
\text { and baby and movies } \\
\end{array}$ & $\begin{array}{l}\text { Domain adaptive } \\
\text { CNN }\end{array}$ & $\begin{array}{l}\text { Accuracy: } \\
\text { Kids and babu } \\
\text { dataset }=94.52 \% \\
\text { Movie dataset }=85.28 \%\end{array}$ \\
\hline $\begin{array}{l}\text { Hamroun, M. et al., } \\
2015\end{array}$ & $\begin{array}{l}\text { Electronics and retail } \\
\text { banking data from } \\
\text { consumer review sites }\end{array}$ & $\begin{array}{l}\text { Customer Intentions } \\
\text { Ontology }\end{array}$ & $\begin{array}{l}\text { Precision and recall: } \\
55.59 \%, 55.28 \% \\
\text { respectively }\end{array}$ \\
\hline \multirow[t]{2}{*}{$\begin{array}{l}\text { (Proposed solution } \\
\text { model 3) }\end{array}$} & \multirow[t]{2}{*}{$\begin{array}{l}\text { Amazon Fresh Reviews } \\
\text { of multiple products. }\end{array}$} & Tailored LightGBM, & $\begin{array}{l}\text { F1-score: } \\
N P I=94.1 \% \\
P P I=92.2 \% \\
U P I=87.4 \%\end{array}$ \\
\hline & & $\begin{array}{l}\text { Tailored NeighLoR } \\
\text { Atouati, S. et al., } \\
2020\end{array}$ & $\begin{array}{l}\text { F1-score: } \\
N P I=95.7 \% \\
P P I=94.4 \% \\
U P I=87.02 \% \\
\end{array}$ \\
\hline
\end{tabular}




\begin{tabular}{|l|l|l|l|}
\hline & LSTM & F1-score: \\
& & $N P I=94.04 \%$ \\
& & $P P I=92.24 \%$ \\
& & $U P I=86.40 \%$ \\
\cline { 3 - 4 } & & CNN & F1-score: \\
& & $N P I=30.6 \%$ \\
& & $P P I=51.7 \%$ \\
& & $U P I=86.4 \%$ \\
\cline { 3 - 4 } & & Logistic regression & F1-score: \\
& & NPI= 48.05\% \\
& & $P P I=50.83 \%$ \\
& & $U P I=96.2 \%$ \\
\hline
\end{tabular}

By looking into the dataset column of table 28, each work exploited different sorts of social media data with the analogous objective to identify the purchase intent of the consumer. Gupta, V. et al., 2014 leveraged analysis on the publicly available questionnaire platforms: Quora and Yahoo! Answers, meanwhile the weakness is, they only performed analysis on a single algorithm (SVM). Dissecting our proposed solution, we can argue that each algorithm's performance varies while computing dynamic behavioral patterns. Ding, $X$. et al, 2015 worked on identifying consumer's intent to purchase by introducing the novel technique of the Consumption Intention Mining Model (CIMM) based on a convolutional neural network $(\mathrm{CNN})$ and achieved $94.52 \%$ accuracy rate. However, their work does not classify the category of intent (Positive or negative) detected. Hamroun, M. et al., 2015 also worked on intention analysis by extracting lexico-semantic patterns based on ontology concepts and relations designed by intentional verbs. They evaluated their novel technique using precision and recall metrics and achieved sub-standard results. For our proposed solution, we collected sufficient data from Amazon Fresh to classify different intent categories reflected in the text. The results column of table 28 concludes that our detailed experimental assessment against state-of-the-art approaches manifested fairly variant and competitive results. 


\section{Chapter 8: Conclusion}

After the experimentation and analysis of our research work, in this chapter, we present the crux of our thesis by discussing the inferences of the distinct models conceived to obtain a pragmatic methodology. Alongside we also present the recommendations for the work extension in the future.

\subsection{Conclusion}

This work consists of three parts: Purchase probability prediction using historical data, Consumer propensity assessment model for Twitter users, and Purchase intension detection model for online reviews. These are the three major contributing solution models of this research work. First, by researching into the transactional data of the Instacart users, we develop a novel ensemble model structure as Purchase probability prediction model, which is evidenced to be effective as compared to other fundamental algorithms and attains an accuracy score of $88.84 \%$. The resulting knowledge could effectively contribute to design strategic promotions to boost the sales of the product high probability rate prediction calculated by model 1 . Second, we apply sentiment analysis on twitter data to build an efficient propensity assessment model by utilizing Random forests and Support vector machines, where former (77.76\%) outperformed the latter (75.62\%) in terms of accuracy metrics. By exploiting virtual social interactions, this model proficiently analyses the demographic placements of prospecting customers with regards to specific market products. Third, we deliver an extension to Atouati, S. et al., 2020's approach by building a model that detects the distinctive three labels of purchase intentions concealed in consumer reviews. The LightGBM algorithm is adapted for this task by assembling the 
customized cost function of Atouati, S. et al., 2020, and tailored loss function. The system framework achieves significantly reliable, consistent, and high prediction metric scores (F1 score: $\mathrm{NPI}=84.47 \%, \mathrm{PPI}=92.2 \%$, $\mathrm{UPI}=87.4 \%$ ) as compared to the base literature's results (Atouati, S. et al., 2020).

While comparing our complete proclivity assessment model (integrated design) with other similar works, we conclude many empirical findings that could further be assessed in the technical research domain of enhancing market strategies. Hence, we can proficiently predict motivated prospects to increase the chances of closing a purchase deal which will lead to overall economic growth (Siegel, E. 2013).

Besides three major contributions as mentioned above, we have other contributions as well: a) We constructed new feature labels based on the original features of the historical data used for model construction. We also provided a comprehensive study that proved the proficiency and effectiveness of our new features as compared to unstructured raw data. b) We developed a flexible and easy-to-access web application based on the integrated model solution as a use case. There is a high potential to improve this web application into a mature product for retailers in the grocery-based market.

Our proposed proclivity assessing solution model is novel in terms of applying marketing mix models that employ the relevant data variables intending to lead higher sales via promotional strategies without increasing costs. Thus, this work demonstrates a significant contribution to a comprehensive evaluation of the retail business and its customers. 


\subsection{Limitations}

As for most research, this analysis involves a few shortcomings which could be discussed in the future report. Primarily, the historical data chosen for model 1 design is for a single year (2017), whereas the model's impact predictions are based on interventional and forward retrospective trials. Due to the unavailability of multiple year's transactional data, the current is therefore subject to biases and confusion that may have influenced our model estimates.

Besides, the integrated model may also be difficult to apply to services where the pricing management of the product is executed already. The high demand for the product on the market is susceptible to many reasons, such as pre-applied promotions and discounts which have not been delivered while assessing the market proclivity. Moreover, the absence of product price in the historical data restricted the model tendencies and hence can be considered as a future directive.

\subsection{Future Work}

Despite achieving a decent metric score for our prediction models, the research still has much potential for future work extension.

First of all, the objective of the historical data model is to predict the selling probability of the products based on the pre-compiled Instacart dataset which poses particular challenges such as shopping trend analysis over multiple years. The design of this model has the potential to leverage the results by including data from multiple years. With a reliable prediction model, we can also incorporate the model with real-time transactional data to provide instant marketing measures. 
While what threats our intention prediction model the most is manual data labeling of distinct intentions reflected in online generated data. The next step is to look into the automated intention label system similar to sentiment labeling models (such as VADER) which will optimize the model by eliminating the obligation of human dependency.

During our intention detection research (model 3), we only involved data from Amazon Fresh for collecting reviews and already got promising results, therefore it proved the reliability of the model for predicting user intentions. Hence, we plan to extend the data for intension classification to incorporate all the different kinds of reviewing websites in accordance with geographical location and see if this approach can further optimize the prediction results.

Moreover, by combining the data visualization techniques mentioned in chapter 2 with our potential use case, we can deliver a visual aid to the retailers for analyzing the market trends and dynamic consumer behavior patterns. 


\section{Glossary}

These definitions are taken from the Oxford dictionary (Oxford online dictionary, 2020):

\begin{tabular}{|c|c|}
\hline Term & Definition \\
\hline Amalgamate & Combine or unite to form one organization or structure \\
\hline Crux & The decisive or most important point. \\
\hline Efficacy & The ability to produce a desired or intended result. \\
\hline Feasible & Possible to do easily or conveniently. \\
\hline Fondness & The state of liking something. \\
\hline Intent & Showing earnest and eager attention. \\
\hline Inclination & $\begin{array}{l}\text { Person's natural tendency or urge to act or feel in a particular } \\
\text { way }\end{array}$ \\
\hline Manifest & $\begin{array}{l}\text { Display or show (a quality or feeling) by one's acts or } \\
\text { appearance; demonstrate. }\end{array}$ \\
\hline Personalization & $\begin{array}{l}\text { The action of designing or producing something to meet } \\
\text { someone's requirements. }\end{array}$ \\
\hline Prediction & Forecast. \\
\hline Predisposition & act in a particular way. \\
\hline Proclivity & An inclination or predisposition toward a particular thing. \\
\hline Prognostication & The action of foretelling or prophesying future events. \\
\hline Propensity & $\begin{array}{l}\text { An inclination or natural tendency to behave in a particular } \\
\text { way. }\end{array}$ \\
\hline Scrutinize & Examine or inspect closely and thoroughly. \\
\hline Segregate & Set apart from the rest or from each other; isolate or divide. \\
\hline Sentiment & A manifestation of the higher or more refined feelings. \\
\hline Triangulate & divide into sections for surveying purposes. \\
\hline Undergird & Provide support or a firm basis for. \\
\hline Voting & $\begin{array}{l}\text { Here, Voting is a machine learning model classifier that trains } \\
\text { on an ensemble of numerous models and predicts an output } \\
\text { (class) based on their highest probability of chosen class as the } \\
\text { output. }\end{array}$ \\
\hline
\end{tabular}




\section{References}

Abdiansah, A., \& Wardoyo, R. (2015). Time complexity analysis of support vector machines (SVM) in LibSVM. International journal computer and application, 128(3), 28-34.

Adhikari, N. C. D., Garg, R., Datt, S., Das, L., Deshpande, S., \& Misra, A. (2017, December). Ensemble methodology for demand forecasting. In 2017 International Conference on Intelligent Sustainable Systems (ICISS) (pp. 846851). IEEE.

Agarwal, R., \& Srikant, R. (1994, September). Fast algorithms for mining association rules. In Proc. of the 20th VLDB Conference (pp. 487-499).

Agrawal, R., Imieliński, T., \& Swami, A. (1993, June). Mining association rules between sets of items in large databases. In Proceedings of the 1993 ACM SIGMOD international conference on Management of data (pp. 207-216).

Airtable-Automate your work (2012), "Automate your work", Accessed from https://airtable.com/integrations. Retrieved on 2020-09-Mar

Airtable Integrations (2016), “Airtable Integrations", Accessed by https://automate.io/integration/airtable. Retrieved on 2020-03-09

Airtable-Product (2012), "Product-Airtable", accessed from https://airtable.com/product. Retrieved on 2020-03-07

Albawi, S., Mohammed, T. A., \& Al-Zawi, S. (2017, August). Understanding of a convolutional neural network. In 2017 International Conference on Engineering and Technology (ICET) (pp. 1-6). IEEE.

Amazon Fresh website (2020), Amazon Fresh official website, accessed from https://www.amazon.com/alm/storefront?almBrandId=QW1hem9uIEZyZXNo, Retrieved on 2020-27-May

Atouati, S., Lu, X., \& Sozio, M. (2020, April). Negative Purchase Intent Identification in Twitter. In Proceedings of The Web Conference 2020 (pp. 2796-2802).

Aung, H. M. S., \& Pa, W. P. (2020, February). Analysis of Word Vector Representation Techniques with Machine-Learning Classifiers for Sentiment Analysis of Public Facebook Page's Comments in Myanmar Text. In 2020 IEEE Conference on Computer Applications (ICCA) (pp. 1-7). IEEE.

Bai, T., Nie, J. Y., Zhao, W. X., Zhu, Y., Du, P., \& Wen, J. R. (2018, June). An attributeaware neural attentive model for next basket recommendation. In The 41st International ACM SIGIR Conference on Research \& Development in Information Retrieval (pp. 1201-1204). 
Barkan, O., \& Koenigstein, N. (2016, September). Item2vec: neural item embedding for collaborative filtering. In 2016 IEEE 26th International Workshop on Machine Learning for Signal Processing (MLSP) (pp. 1-6). IEEE.

Beautiful Soup (2020). Beautiful Soup from Python library, accessed from https://pypi.org/project/beautifulsoup4/, Retrieved on 2020-06-02

Breiman, L. (2001). Random Forests Machine Learning, vol. 45.

Brownlee, J., (2019), "Binary Classification Tutorial with the Keras Deep Learning Library", Accessed from https://machinelearningmastery.com/binaryclassification-tutorial-with-the-keras-deep-learning-library/. Retrieved on 202002-02

Buser, D. C. (2007, January). Context-Based Recommender Systems in Conventional Grocery--An Economic Analysis. In 2007 40th Annual Hawaii International Conference on System Sciences (HICSS'07) (pp. 168b-168b). IEEE.

Caruana, R., Munson, A., \& Niculescu-Mizil, A. (2006, December). Getting the most out of ensemble selection. In Sixth International Conference on Data Mining (ICDM'06) (pp. 828-833). IEEE.

CatBoost (2014), accessed from https://tech.yandex.com/catboost/. Retrieved on 2020$01-23$

Chandra, Y., \& Jana, A. (2020, March). Sentiment Analysis using Machine Learning and Deep Learning. In 2020 7th International Conference on Computing for Sustainable Global Development (INDIACom) (pp. 1-4). IEEE.

Chaudhuri, A., \& Holbrook, M. B. (2001). The chain of effects from brand trust and brand affect to brand performance: the role of brand loyalty. Journal of marketing, 65(2), 81-93.

Chauhan, N. S. (2020), "Introduction to Artificial neural network", Accessed from https:/towardsdatascience.com/introduction-to-artificial-neural-networks- ann1aea15775ef9. Retrieved on 2020-01-29

Chawla, N. V., Bowyer, K. W., Hall, L. O., \& Kegelmeyer, W. P. (2002). SMOTE: synthetic minority over-sampling technique. Journal of artificial intelligence research, 16, 321-357.

Chen, M. C., Chiu, A. L., \& Chang, H. H. (2005). Mining changes in customer behavior in retail marketing. Expert Systems with Applications, 28(4), 773-781.

Chen, T., \& Guestrin, C. (2016, August). Xgboost: A scalable tree boosting system. In Proceedings of the 22nd acm sigkdd international conference on knowledge discovery and data mining (pp. 785-794).

Chen, T., Hong, L., Shi, Y., \& Sun, Y. (2017). Joint text embedding for personalized content-based recommendation. arXiv preprint arXiv:1706.01084. 
Cherkassky, V., Krasnopolsky, V., Solomatine, D. P., \& Valdes, J. (2006).

Computational intelligence in earth sciences and environmental applications: Issues and challenges. Neural Networks, 19(2), 113-121

Chrysochou, P. (2017). Consumer behavior research methods. In Consumer Perception of Product Risks and Benefits (pp. 409-428). Springer, Cham.

Convolutional neural network (2020), accessed from https://en.wikipedia.org/wiki/Convolutional_neural_network. Retrieved on 202002-02

Da Silva, N. F., Hruschka, E. R., \& Hruschka Jr, E. R. (2014). Tweet sentiment analysis with classifier ensembles. Decision Support Systems, 66, 170-179.

Deloitte Touche Tohmatsu Limited. Global Powers of Retailing 2003 (for FY2001 data), 2008 (FY2006 data), 2013 (FY2011 data) and 2018 (FY2016 data).

Dhiraj, K. (2019), "How to create a custom loss function in Keras", accessed from https:/heartbeat.fritz.ai/how-to-create-a-custom-loss-function-in-keras637bd312e9ab, Retrieved on 2020-06-15

Ding, X., Liu, T., Duan, J., \& Nie, J. Y. (2015, February). Mining user consumption intention from social media using domain adaptive convolutional neural network. In Twenty-Ninth AAAI Conference on Artificial Intelligence.

Domingos, P. (2012). A few useful things to know about machine learning. Communications of the ACM, 55(10), 78-87.

Dou, X. (2020, April). Online Purchase Behavior Prediction and Analysis Using Ensemble Learning. In 2020 IEEE 5th International Conference on Cloud Computing and Big Data Analytics (ICCCBDA) (pp. 532-536). IEEE.

Drummond, C., \& Holte, R. (2000). Explicitly Representing Expected Cost: An Alternative to ROC Representation. In Proceedings of the Sixth ACM SIGKDD International Conference on Knowledge Discovery and Data Mining, pp. 198-207 Boston. ACM.

Efrat, A. R., \& Gernowo, R. (2020, April). Consumer purchase patterns based on market basket analysis using apriori algorithms. In Journal of Physics: Conference Series (Vol. 1524, No. 1, p. 012109). IOP Publishing.

Facebook website (2020), Facebook official website, accessed from https://www.facebook.com/, Retrieved on 2020-11-Feb

False Positives and False Negatives (2020), accessed from https://en.wikipedia.org/wiki/False_positives_and_false_negative". Retrieved on 2020-02-24

Fernandes, R. F., \& Teixeira, C. M. (2015). Using clickstream data to analyze online purchase intentions. 
Garbade, Dr. M. J. (2018). "Simple neural network python", Accessed from https://www.kdnuggets.com/2018/10/simple-neural-network-python.html. Retrieved on 2020-01-29

Gatzioura, A., \& Sànchez-Marrè, M. (2014). A case-based recommendation approach for market basket data. IEEE Intelligent systems, 30(1), 20-27.

Giering, M. (2008). Retail sales prediction and item recommendations using customer demographics at store level. ACM SIGKDD Explorations Newsletter, 10(2), 8489 .

Giudici, P., \& Passerone, G. (2002). Data mining of association structures to model consumer behaviour. Computational Statistics \& Data Analysis, 38(4), 533-541.

Gokulakrishnan, B., Priyanthan, P., Ragavan, T., Prasath, N., \& Perera, A. (2012, December). Opinion mining and sentiment analysis on a twitter data stream. In International Conference on Advances in ICT for Emerging Regions (ICTer2012) (pp. 182-188). IEEE.

Grbovic, M., \& Cheng, H. (2018, July). Real-time personalization using embeddings for search ranking at airbnb. In Proceedings of the 24th ACM SIGKDD International Conference on Knowledge Discovery \& Data Mining (pp. 311-320).

Grbovic, M., Radosavljevic, V., Djuric, N., Bhamidipati, N., Savla, J., Bhagwan, V., \& Sharp, D. (2015, August). E-commerce in your inbox: Product recommendations at scale. In Proceedings of the 21th ACM SIGKDD international conference on knowledge discovery and data mining (pp. 1809-1818).

Grover, P. (2018) “Custom Loss Functions for Gradient Boosting”, accessed from https://towardsdatascience.com/custom-loss-functions-for-gradient-boostingf79c1b40466d, Retrieved on 2020-08-22

Gulin, A. et al., (2020). Python Usage Examples: CatBoostClassification, accessed from https://catboost.ai/docs/concepts/python-usages-examples.html. Retrieved on 2020-01-23

Gupta, V., Varshney, D., Jhamtani, H., Kedia, D., \& Karwa, S. (2014, May). Identifying purchase intent from social posts. In Eighth International AAAI Conference on Weblogs and Social Media.

Hamroun, M., Gouider, M. S., \& Said, L. B. (2015). Customer intentions analysis of twitter based on semantic patterns. In The 11th International Conference on Semantics, Knowledge and Grids (pp. 2-6).

Han, J., Pei, J., \& Yin, Y. (2000). Mining frequent patterns without candidate generation. ACM sigmod record, 29(2), 1-12. 
Han, K. X., Chien, W., Chiu, C. C., \& Cheng, Y. T. (2020). Application of Support Vector Machine (SVM) in the Sentiment Analysis of Twitter DataSet. Applied Sciences, 10(3), 1125.

Hanke, J., Hauser, M., Dürr, A., \& Thiesse, F. (2018). Redefining the Offline Retail Experience: Designing Product Recommendation Systems for Fashion Stores.

Haque, R., Ramadurai, A., Hasanuzzaman, M., \& Way, A. (2019). Mining Purchase Intent in Twitter. Computación y Sistemas, 23(3).

Harrington, P. (2012). Machine learning in action. Manning Publications Co.

Hegde, Y., \& Padma, S. K. (2017, January). Sentiment analysis using random forest ensemble for mobile product reviews in Kannada. In 2017 IEEE 7th International Advance Computing Conference (IACC) (pp. 777-782). IEEE.

Heroku - Salesforce.com, Inc (1999), “The best way to build apps with open languages and frameworks", Accessed from https://developer.salesforce.com/platform/heroku. Retrieved on 2020-03-07

Hochreiter, S., \& Schmidhuber, J. (1997). Long short-term memory. Neural computation, 9(8), 1735-1780.

Honnibal, M. (2015), accessed form https://spacy.io/. Retrieved on 2020-06-03

Howal, S. (2017). "Ensemble Learning in Machine Learning | Getting Started”. Accessed from https://towardsdatascience.com/ensemble-learning-in-machine-learninggetting-started-4ed85eb38e00, retrievd on 2020-08-14

Howard, A. G. (2013). Some improvements on deep convolutional neural network-based image classification. arXiv preprint arXiv:1312.5402.

Hübner, A. H., Kuhn, H., \& Sternbeck, M. G. (2013). Demand and supply chain planning in grocery retail: an operations planning framework. International Journal of Retail \& Distribution Management.

Huilgol, P (2019), “Accuracy vs. F1-Score”. Accessed from https://medium.com/analytics-vidhya/accuracy-vs-f1-score6258237beca2\#: :text=Accuracy\%20is\%20used\%20when $\% 20$ the, as $\% 20 \mathrm{in} \% 20^{\text {th }}$ e\%20above\%20case, Retrieved on 2020-08-14

Instacart website (2020), Instacart official website, accessed from https://www.instacart.com/, Retrieved on 2020-02-11

Instacart Online Grocery Shopping Dataset (2017), accessed from https://www.instacart.com/datasets/grocery-shopping-2017, Retrieved on September 5, 2019.

Instacart Privacy policy (2017), accessed from https:/www.instacart.com/privacy on September 5, 2019 
James Loy (2018), "How to build your own neural network from scratch in python", Accessed from https://towardsdatascience.com/how-to-build-your-own-neuralnetwork-from-scratch-in-python-68998a08e4f6. Retrieved on 2020-01-29

Jiang, Y., Tong, G., Yin, H., \& Xiong, N. (2019). A Pedestrian Detection Method Based on Genetic Algorithm for Optimize XGBoost Training Parameters. IEEE Access, 7, 118310-118321.

Joshi, R., \& Tekchandani, R. (2016, August). Comparative analysis of Twitter data using supervised classifiers. In 2016 International Conference on Inventive Computation Technologies (ICICT) (Vol. 3, pp. 1-6). IEEE.

Jupyter notebook (2020), "Project Jupyter - About Us". accessed from https://jupyter.org/about. Retrieved on 2019-12-12.

Kabasakal, İ. (2020). Understanding Shopping Behaviors With Category-and BrandLevel Market Basket Analysis. In Tools and Techniques for Implementing International E-Trading Tactics for Competitive Advantage (pp. 242-267). IGI Global.

Kaneko, Y., \& Yada, K. (2016, December). A deep learning approach for the prediction of retail store sales. In 2016 IEEE 16th International Conference on Data Mining Workshops (ICDMW) (pp. 531-537). IEEE.

Kazachok, M. (2020), "CatBoost with gridsearch-cv", Accessed from https://www.kaggle.com/miklgr500/catboost-with-gridsearch-cv. Retrieved on 2020-01-23

Ke, G., Meng, Q., Finley, T., Wang, T., Chen, W., Ma, W., ... \& Liu, T. Y. (2017). Lightgbm: A highly efficient gradient boosting decision tree. In Advances in neural information processing systems (pp. 3146-3154).

Keller, T., \& Raffelsieper, M. (2014, October). Cosibon: an E-commerce like platform enabling bricks-and- mortar stores to use sophisticated product recommender systems. In Proceedings of the 8th ACM Conference on Recommender Systems (pp. 367-368).

Kelly, F. (2002) "Implementing an EIS", Accessed from http://www.dssresources.com/newsletters/66.php, Retrieved on 2020-02-11

Kim, H. W., \& Gupta, S. (2009). A comparison of purchase decision calculus between potential and repeat customers of an online store. Decision Support Systems, 47(4), 477-487.

Kiranyaz, S., Avci, O., Abdeljaber, O., Ince, T., Gabbouj, M., \& Inman, D. J. (2019). 1D convolutional neural networks and applications: A survey. arXiv preprint arXiv:1905.03554. 
Kleinbaum, D. G., Dietz, K., Gail, M., Klein, M., \& Klein, M. (2002). Logistic regression. New York: Springer-Verlag.

Koehrsen, W. (2018). "Beyond Accuracy: Precision and Recall", Accessed from https://towardsdatascience.com/ beyond-accuracy-precision-and-recall3 da06bea9f6c. Retrieved on 2020-02-23

Koehrsen, W. (2017). "Random Forest in Python", Accessed from https://towardsdatascience.com/random-forest-in-python-24d0893d51c0. Retrieved on 2020-01-23

Kouki, P., Fountalis, I., Vasiloglou, N., Yan, N., Ahsan, U., Jadda, K. A., \& Qu, H. (2019, September). Product collection recommendation in online retail. In Proceedings of the 13th ACM Conference on Recommender Systems (pp. 486490).

Krzyk, K. (2018), “Coding Deep Learning for Beginners — Linear Regression (Part 2): Cost Function", accessed from https://towardsdatascience.com/coding-deeplearning-for-beginners-linear-regression-part-2-cost-function-49545303d29f, Retrieved on 2020-06-04

Kumar, A. (2018), "ML Metrics: Sensitivity vs specificity”, Accessed from https://dzone.com/articles/ml-metrics-sensitivity-vs-specificity-difference. Retrieved on 2020-02-23

Kumar, A., Kabra, G., Mussada, E. K., Dash, M. K., \& Rana, P. S. (2019). Combined artificial bee colony algorithm and machine learning techniques for prediction of online consumer repurchase intention. Neural Computing and Applications, 31(2), 877-890.

Kumar, V., \& Subba, B. (2020, February). A TfidfVectorizer and SVM based sentiment analysis framework for text data corpus. In 2020 National Conference on Communications (NCC) (pp. 1-6). IEEE.

Kumari, P., \& Haider, M. T. U. (2020, January). Sentiment Analysis on Aadhaar for Twitter Data - A Hybrid. In Proceeding of International Conference on Computational Science and Applications: ICCSA 2019 (p. 309). Springer Nature.

Le, D. T., Lauw, H. W., \& Fang, Y. (2017). Basket-sensitive personalized item recommendation. IJCAI.

Lessmann, S., Haupt, J., Coussement, K., \& De Bock, K. W. (2019). Targeting customers for profit: An ensemble learning framework to support marketing decisionmaking. Information Sciences.

Li, J., Burke, E. K., \& Qu, R. (2011). Integrating neural networks and logistic regression to underpin hyper-heuristic search. Knowledge-Based Systems, 24(2), 322-330. 
Li, M., Dias, B. M., Jarman, I., El-Deredy, W., \& Lisboa, P. J. (2009, June). Grocery shopping recommendations based on basket-sensitive random walk. In Proceedings of the 15th ACM SIGKDD international conference on Knowledge discovery and data mining (pp. 1215-1224).

Li, Y., Xu, L., Tian, F., Jiang, L., Zhong, X., \& Chen, E. (2015, June). Word embedding revisited: A new representation learning and explicit matrix factorization perspective. In Twenty-Fourth International Joint Conference on Artificial Intelligence.

Liang, P. W., \& Dai, B. R. (2013, June). Opinion mining on social media data. In 2013 IEEE 14th International Conference on Mobile Data Management (Vol. 2, pp. 9196). IEEE.

Liaw, A., \& Wiener, M. (2002). Classification and regression by randomForest. R news, 2(3), 18-22.

Lin, W., Alvarez, S. A., \& Ruiz, C. (2002). Efficient adaptive-support association rule mining for recommender systems. Data mining and knowledge discovery, 6(1), 83-105.

Louppe, G. (2014). Understanding random forests: From theory to practice. arXiv preprint arXiv: 1407.7502 .

Lu, H., Han, J., \& Feng, L. (1998, June). Stock movement prediction and n-dimensional inter-transaction association rules. In Proc. of the ACM SIGMOD Workshop on Research Issues in Data Mining and Knowledge Discovery (p. 12).

Makice, K. (2009). Twitter API: Up and running: Learn how to build applications with the Twitter API. " O'Reilly Media, Inc.".

Malik, U., (2020), "Random forest algorithm with python and scikit learn", Accessed from https://stackabuse.com/random-forest-algorithm-with-python-and-scikitlearn/. Retrieved on 2020-23-Jan

Manyika, J. (2011), Big data: The next frontier for innovation, competition, and productivity. http://www.mckinsey.com/Insights/MGI/Research/Technology_and _Innovation/Big_data_The_next_frontier_for_innovation.

MaxMinScaler (2020), MaxMinScaler processing by sklearn python library, access from https://scikitlearn.org/stable/modules/generated/sklearn.preprocessing.MinMaxSc aler.html, retrieved on 2020- 12-Jun

MediaPost (2018), accessed from https://www.mediapost.com/publications/article/291358/90-of-todays-datacreated-in-two-years.html, Retrieved on 2020-02-11

Miglautsch, J. R. (2000). Thoughts on RFM scoring. Journal of Database Marketing \& Customer Strategy Management, 8(1), 67-72. 
Mikolov, T., Sutskever, I., Chen, K., Corrado, G. S., \& Dean, J. (2013). Distributed representations of words and phrases and their compositionality. In Advances in neural information processing systems (pp. 3111-3119).

Mohan, A., Abdelrazeq, A., \& Hees, F. (2019, June). Recommendation System in Business Intelligence Solutions for Grocery shops: Challenges and Perspective. In Proceedings of the 2019 3rd International Conference on E-commerce, EBusiness and E-Government (pp. 53-57).

Narkhede, S., (2018), "Understanding ROC Curve", Accessed from https://towardsdatascience.com/understanding-auc-roc-curve-68b2303cc9c5. Retrieved on 2020-13-Feb

NLTK (2020), Natural Language Toolkit, accessed from https://www.nltk.org/, Retrieved on 2020-05-28

NLTK.tag package (2020), accessed from https://www.nltk.org/api/nltk.tag.html, Retrieved on 2020-06-01

Oxford Online Dictionary, accessed from https://en.oxforddictionaries.com/definition, Retrieved on 2020-08-13

Padmanabhan, B., Zheng, Z., \& Kimbrough, S. O. (2001, August). Personalization from incomplete data: what you don't know can hurt. In Proceedings of the seventh ACM SIGKDD international conference on Knowledge discovery and data mining (pp. 154-163).

Pak, A., \& Paroubek, P. (2010, May). Twitter as a corpus for sentiment analysis and opinion mining. In LREc (Vol. 10, No. 2010, pp. 1320-1326).

Pandey, P. (2018), "Simplifying Sentiment Analysis using VADER in Python (on Social Media Text)", Accessed from https://medium.com/analyticsvidhya/simplifying-social-media-sentiment-analysis-using-vader-in-pythonf9e6ec6fc52f, Retrieved on 2020-05-28.

Part-of-speech tag module from NLTK library (2020), accessed from https://www.nltk.org/api/nltk.tag.html, retrieved on 2020-06-03

Pathak, M. (2020), "XGboost in python", Accessed from https://www.datacamp.com/community/tutorials/xgboost-in-python. Retrieved on 2020-01-23

Patil, H., \& Jha, P. (2020), Analysing Real Time Big Social Data and Modelling Emotions in Social Media (Twitter).

Paul, S. (2018), "Ensemble Learning in Python", Accessed from https://www.datacamp.com/community/tutorials/ensemble-learning-python. Retrieved on 2020-04-12 
Pennington, J., Socher, R., \& Manning, C. D. (2014, October). Glove: Global vectors for word representation. In Proceedings of the 2014 conference on empirical methods in natural language processing (EMNLP) (pp. 1532-1543).

Peter, J. P., Olson, J. C., \& Grunert, K. G. (1999). Consumer behaviour and marketing strategy. London, UK:: McGraw-hill.

Phienthrakul, T., Kijsirikul, B., Takamura, H., \& Okumura, M. (2009, December). Sentiment classification with support vector machines and multiple kernel functions. In International Conference on Neural Information Processing (pp. 583-592). Springer, Berlin, Heidelberg.

PICO - What is False Positive rate? (2020), Accessed from https://www.pico.net/kb/what-is-a-false-positive-rate. Retrieved on 2020-02-24

Powers, D. M. (2011). Evaluation: from precision, recall and F-measure to ROC, informedness, markedness and correlation.

Prasad, R., Pandey, A., Singh, K. P., Singh, V. P., Mishra, R. K., \& Singh, D. (2012). Retrieval of spinach crop parameters by microwave remote sensing with back propagation artificial neural networks: A comparison of different transfer functions. Advances in space research, 50(3), 363-370.

Rakotomalala, R. (2005). "TANAGRA: a free software for research and academic purposes".

Raorane, A. A., Kulkarni, R. V., \& Jitkar, B. D. (2012). Association rule-extracting knowledge using market basket analysis. Research Journal of Recent Sciences

Rigby, D.K (2019). “The future of shopping” - Harward Business Review, accessed from https://hbr.org/2011/12/the-future-of-shopping, Retrieved in 2020-08-16

Safitri, A. (2019). Consumer Behavior Analysis using Apriori Algorithm. International Journal of Information Technology and Business, 1(2), 5-5.

Sagin, A. N., \& Ayvaz, B. (2018). Determination of Association Rules with Market Basket Analysis: Application in the Retail Sector. Southeast Europe Journal of Soft Computing, 7(1).

Saha, A. K., Gupta, R. P., \& Arora, M. K. (2002). GIS-based landslide hazard zonation in the Bhagirathi (Ganga) valley, Himalayas. International journal of remote $\mathrm{s}$ ensing, 23(2), 357-369.

Sakar, C. O., Polat, S. O., Katircioglu, M., \& Kastro, Y. (2019). Real-time prediction of online shoppers' purchasing intention using multilayer perceptron and LSTM recurrent neural networks. Neural Computing and Applications, 31(10), 68936908.

Salesforce Platform Lightning (1999), "Salesforce Platform Lightning: Building Blocks of Better Apps", Accessed from 
https://www.salesforce.com/ca/products/platform/lightning/. Retrieved on 202003-7

Sarwar, B. M., Karypis, G., Konstan, J., \& Riedl, J. (2002, December). Recommender systems for large-scale e-commerce: Scalable neighborhood formation using clustering. In Proceedings of the fifth international conference on computer and information technology (Vol. 1, pp. 291-324).

Savasere, A., Omiecinski, E. R., \& Navathe, S. B. (1995). An efficient algorithm for mining association rules in large databases. Georgia Institute of Technology.

Schafer, J. B., Konstan, J. A., \& Riedl, J. (2001). E-commerce recommendation applications. Data mining and knowledge discovery, 5(1-2), 115-153.

Sharma, A. (2017), "Convolutional Neural Networks in Python with Keras", Accessed from https://www.datacamp.com/community/tutorials/convolutional-neuralnetworks-python. Retrieved on 2020-02-02

Sharma, A. (2020). Confusion matrix in machine learning, accessed from https://www.geeksforgeeks.org/confusion-matrix-machine-learning/. Retrieved on 2020-02-12

Shearer, C. (2000). The CRISP-DM: The new blueprint for data mining. Journal of data warehousing, 5(4).

Shostak, T., (2019), "Using Machine learning to reduce False positive while combating attacks: Part 1", Accessed from https://www.reblaze.com/blog/usingmachine-learning-reduce-false-positives-combating-attacks-part-1/. Retrieved on 2020-02-24

Siegel, E. (2013). Predictive analytics: The power to predict who will click, buy, lie, or die. John Wiley \& Sons.

Singh, A. (2018), "A Comprehensive Guide to Ensemble Learning (with Python codes)", Accessed from https://www.analyticsvidhya.com/blog/2018/06/comprehensive-guide-forensemble-models/. Retrieved on 2020-04-12

Singh, N., \& Rao, S. (2014). Ensemble learning for large-scale workload prediction. IEEE Transactions on Emerging Topics in Computing, 2(2), 149-165.

Statistical view of Pointwise mutual information (2020), accessed from https://en.wikipedia.org/wiki/Pointwise_mutual_information, Retrieved on 202006-03

Suharjo, R. A., \& Wibowo, A. (2020). Customer Relationship Management in Retail Using Double Association Rule. International Journal, 8(5). 
Sullivan, Dr. L. (2020), "Confidence intervals", accessed from http://sphweb.bumc.bu.edu/otlt/MPHModules/BS/BS704_Confidence_Intervals/B S704_Confidence_Intervals_print.html, Retrieved on 2020-06-03

Support Vector Machines from Scikit-learn library (2007), accessed from https://scikitlearn.org/stable/modules/svm.html, Retrieved on 2020-05-30

Svetnik, V., Liaw, A., Tong, C., Culberson, J. C., Sheridan, R. P., \& Feuston, B. P. (2003). Random forest: a classification and regression tool for compound classification and QSAR modeling. Journal of chemical information and computer sciences, 43(6), 1947-1958.

Swalin, A. (2020) "catboost-vs-light-gbm-vs-xgboost", Accessed from https://towardsdatascience.com/catboost-vs-light-gbm-vs-xgboost-5f93620723db. Retrieved on 2020-01-23

Tahiri, N., Mazoure, B., \& Makarenkov, V. (2019). An intelligent shopping list based on the application of partitioning and machine learning algorithms.

TfidfVectorizer Class from Scikit-learn library (2007), accessed from https://scikitlearn.org/stable/modules/generated/sklearn.feature_extraction.text.TfidfVectorizer .html, Retrieved on 2020-05-29

Training and testing data splitting module from Scikit-learn library (2007), accessed from https://scikit_learn.org/stable/modules/generated/sklearn.model_selection.train_te st_split.html, Retrieved on 2020-05-29

Trofimov, I. (2018). Inferring complementary products from baskets and browsing sessions. arXiv preprint arXiv:1809.09621.

Tweetcloud website (2010), Accessed from http://tweetcloud.icodeforlove.com/. Retrieved on 2020-02-11

Twitter website (2020), Twitter official website, accessed from https://www.twitter.com/, Retrieved on 2020-02-11

Twitter sentiment analysis using Python (2020), accessed from https://www.geeksforgeeks.org/twitter-sentiment-analysis-using-python/. Retrieved on 2020-01-16

Tyagi, K. (2020). ML | Handling Imbalanced data using SMOTE, accessed from https://www.geeksforgeeks.org/ml-handling-imbalanced-data-with-smote-andnear-miss-algorithm-in-python/. Retrieved on 2020-01-27

Tyre, A. J., Tenhumberg, B., Field, S. A., Niejalke, D., Parris, K., \& Possingham, H. P. (2003). Improving precision and reducing bias in biological surveys: estimating false-negative error rates. Ecological Applications, 13(6), 1790-1801. 
VADER sentiment analyzer (2020), accessed from https://www.nltk.org/_modules/nltk/sentiment/vader.html, Retrieved on 2020-0528

Vakili, M., Ghamsari, M., \& Rezaei, M. (2020). Performance Analysis and Comparison of Machine and Deep Learning Algorithms for IoT Data Classification. arXiv preprint arXiv:2001.09636.

Vasile, F., Smirnova, E., \& Conneau, A. (2016, September). Meta-prod2vec: Product embeddings using side-information for recommendation. In Proceedings of the 10th ACM Conference on Recommender Systems (pp. 225-232).

Vatrapu, R., Hussain, A., Lassen, N. B., Mukkamala, R., Flesch, B., \& Madsen, R.(2015). Social set analysis: four demonstrative case studies. Proceedings of the 2015 International Conference on Social Media \& Society.

Verbeke, W., Dejaeger, K., Martens, D., Hur, J., \& Baesens, B. (2012). New insights into churn prediction in the telecommunication sector: A profit driven data mining approach. European Journal of Operational Research, 218(1), 211-229.

Videla Cavieres, I. F. (2014). Characterization and completation of the customer data from a retail company using graph mining techniques.

Walter, F. E., Battiston, S., Yildirim, M., \& Schweitzer, F. (2012). Moving recommender systems from on-line commerce to retail stores. Information Systems and eBusiness Management, 10(3), 367-393.

Walter, S. D. (2005). The partial area under the summary ROC curve. Statistics in medicine, 24(13), 2025-2040

Wan, M., Wang, D., Liu, J., Bennett, P., \& McAuley, J. (2018, October). Representing and recommending shopping baskets with complementarity, compatibility and loyalty. In Proceedings of the 27th ACM International Conference on Information and Knowledge Management (pp. 1133-1142).

Wang, P., Guo, J., \& Lan, Y. (2014, November). Modeling retail transaction data for personalized shopping recommendation. In Proceedings of the 23rd ACM international conference on conference on information and knowledge management (pp. 1979-1982).

Wirth, R., \& Hipp, J. (2000, April). CRISP-DM: Towards a standard process model for data mining. In Proceedings of the 4th international conference on the practical applications of knowledge discovery and data mining (pp. 29-39). London, UK: Springer-Verlag.

Wölbitsch, M., Walk, S., Goller, M., \& Helic, D. (2019, June). Beggars can't be choosers: Augmenting sparse data for embedding-based product recommendations in retail stores. In Proceedings of the 27th ACM Conference on User Modeling, Adaptation and Personalization (pp. 104-112). 
Wong, W. K., Leung, S. Y. S., Guo, Z. X., Zeng, X. H., \& Mok, P. Y. (2012). Intelligent product cross-selling system with radio frequency identification technology for retailing. International Journal of Production Economics, 135(1), 308-319.

WordNet Lemmatizer (2020), A module from NLTK library, accessed from https://www.nltk.org/_modules/nltk/stem/wordnet.html, retrieved on 2020-06-03

Wu, Z., \& Palmer, M. (1994). V Verbs semantics and lexical selection. In Proceedings of the 32nd annual meeting on Association for Computational Linguistics (pp. 133138).

XingFen, W., Xiangbin, Y., \& Yangchun, M. (2018, December). Research on user consumption behavior prediction based on improved XGBoost algorithm. In 2018 IEEE International Conference on Big Data (Big Data) (pp. 4169-4175). IEEE.

Yazdizadeh, A., Patterson, Z., \& Farooq, B. (2019). Ensemble convolutional neural networks for mode inference in smartphone travel survey. IEEE Transactions on Intelligent Transportation Systems, 21(6), 2232-2239.

Yu, F., Liu, Q., Wu, S., Wang, L., \& Tan, T. (2016, July). A dynamic recurrent model for next basket recommendation. In Proceedings of the 39th International ACM SIGIR conference on Research and Development in Information Retrieval (pp. 729-732).

Zaleski, O., and Huet, E. (2018), "With fresh funding, Apoorva Mehta is taking the grocery delivery fight to Amazon". The Economic Times. Retrieved 2019-07-09

Zapier (2011), “Airtable Integrations", accessed by https://zapier.com/apps/airtable/integrations. Retrieved on 2020-03-09

Zarina, I., (2020) “A simple XGBoost tutorial using Iris dataset”, Accessed from https://www.kdnuggets.com/2017/03/simple-xgboost-tutorial-iris-dataset.html. Retrieved on 2020-23-Jan 